\title{
An Atlas of Thermal Data For Biomass and Other Fuels
}

Siddhartha Gaur

Thomas B. Reed

Colorado School of Mines

Golden, CO 80401

NREL technical monitor: Tom Milne

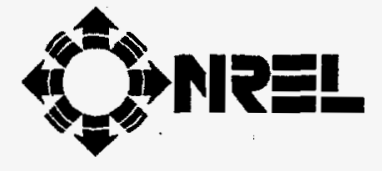

National Renewable Energy Laboratory

1617 Cole Boulevard

Golden, Colorado 80401-3393

A national laboratory of the U.S. Department of Energy Managed by the Midwest Research Institute for the U.S. Department of Energy under Contract No. DE-AC36-83CH10093

Prepared under Subcontract No. HZ-2-12083-1

June 1995

DISTRIBUTION OF THIS DOCUMENT IS UNLIMITED 
This publication was reproduced from the best available camera-ready copy subnitted by the subcontractor and received no editorial review at NREL.

\section{NOTICE}

This report was prepared as an account of work sponsored by an agency of the United States government. Neither the United States government nor any agency thereof, nor any of their employees, makes any warranty, express or implied, or assumes any legal liability or responsibility for the accuracy, completeness, or usefulness of any information, apparatus, product, or process disciosed, or represents that its use would not infringe privately owned rights. Reference herein to any specific commercial product, process, or service by trade name, trademark, manufacturer, or otherwise does not necessarily constitute or imply its endorsement, recommendation, or favoring by the United States government or any agency thereof. The views and opinions of authors expressed herein do not necessarily state or reflect those of the United States government or any agency thereof.

Available to DOE and DOE contractors from:

Office of Scientific and Technical Information (OSTI)

P.O. Box 62

Oak Ridge, TN 37831

Prices available by calling (615) 576-8401

Available to the public from:

National Technical Information Service (NTIS)

U.S. Department of Commerce

5285 Port Royal Road

Springfield, VA 22161

(703) $487-4650$ 


\section{DISCLAIMER}

Portions of this document may be illegible in electronic image products. Images are produced from the best available original document. 


\section{ABOUT THE ATLAS}

Biomass is recognized as a major source of renewable energy. It has been predicted that biomass as the potential to supply $20 \%$ of U.S. Energy by the year 2000 [OTA, 1980]. Recently a major review has summarized ten years of work on biomass thermal conversion [Stevens, 1994].

In order to convert biomass energy to more useful forms, it is necessary to have accurate scientific data on the thermal properties of biomass. We have written this Atlas to supply a uniform source o that information.

Some thermal data such as proximate and ultimate analysis have been used routinely in evaluating coal, biomass and other fuels for over a century. In the last few decades additional Thermal analysis (TA) tools such as thermogravimetry, differential thermal analysis, thermo mechanical analysis etc. have become more important. The data obtained from these techniques can provide useful information in terms of reaction mechanism, kinetic parameters, thermal stability, phase transformation, heat of reaction, etc. for gas-solid and gas-liquid systems. Unfortunately, there are no ASTM standards set for the collection of these types of data using TA techniques and therefore, different investigators use different conditions which suit their requirements for measuring this thermal data. As a result, the information obtained from different laboratories is not comparable. This Atlas provides the ability to compare new laboratory results with a wide variety of related data available in the literature and helps ensure consistency in using these data.

The first book on thermal analysis "Thermografica" was published by Berg, Nikolaer and Rode in 1944. Since that time a large number of books and articles have appeared and there is now an International Confederation on Thermal Analysis (ICTA) with annual meetings, publications and recommendations. Concurrently, a number of companies have produced a wide variety of apparati for acquiring thermal data and powerful software to go along with it.

An Atlas of Thermoanalytical Curves prepared by Liptay was published in Hungary [Liptay, 1972]. This Atlas contains primarily information for inorganic samples. There is however, no collection of such information for organic materials or specifically for fuel samples. The preparation of this Atlas is an attempt to bridge this gap in thermal analysis literature.

Thermal analysis data is useful both for researchers and practicing engineers. For researchers the thermal analysis data provide the information for the identification of different reaction mechanism, determination of kinetic parameters and optimization of conditions to favor one reaction over the other. The specific temperatures at which various heterogeneous reactions occur, their reaction rates and the energies involved in these reactions are invaluable information for engineers involved in system design. 
This Atlas presents thermogravimetric (TG) and differential thermal analysis (DTA) data taken under comparable conditions on a wide variety of organic materials such as biomass fuels, coal and municipal solid wastes making it easy to find and compare data on this large variety of energy sources. Some data has been collected by changing the operating conditions to show the effects of parameters such as particle size, heating rate etc. on the decomposition pattern of a solid sample. This information can then be used to provide a guideline for the extrapolation of the bulk of the data and data previously available in the literature but obtained at different conditions.

In Thermogravimetric (TG) analysis the weight of a sample is recorded as a function of time or temperature while heating the sample in a pre-set time-temperature program. Experimental data for a typical thermogram on a $15 \mathrm{mg}$ sample of Western red cedar is shown in Fig. 1 as recorded by the (Seiko SSC 5200) apparatus. The heating program used on this and most other samples is shown in the upper right corner. The sample is heated in a flowing nitrogen atmosphere. The actual recorded temperature $T$ and the recorded weight, TG, are shown as a function of time. The loss of water below $125^{\circ} \mathrm{C}$ and the loss of volatiles in the range $200^{\circ} \mathrm{C}$ to $400^{\circ} \mathrm{C}$ is clearly seen. At $900^{\circ} \mathrm{C}$ air is admitted to the system and the charcoal is burned, leaving ash. The difference in temperature between the sample pan and an inert sample is shown on the DTA curve in $\mu \mathrm{V}$. The endothermic loss of water and volatile material are represented by negative peaks, while the carbon combustion is a positive peak.

These data has been selected and analyzed using TG software in Fig. 2 to show the most important features. The TG curve shows weight loss. The Differential Thermogravimetric Analysis, DTG curve shows the rate of weight loss. Thedifferential thermal analysis, DTA, curve shows the heat required for volatilization.

The sidebar on each thermogram summarizes the numerical data on each graph and includes heating rate, moisture free sample weight, TG data, weight \% remaining at $650^{\circ} \mathrm{C}$ and the area under the DTA peak, proportional to $\Delta \mathrm{H}_{\mathrm{vol}}$, the heat of volatilization and decomposition.

The temperature at which there is the first sign of weight loss in the sample being heated is shown as $T_{\text {initial }}$. $T_{\text {start point }}$ is shown as the beginning of the $S$ curve as obtained from the method for determining glass transition temperature. $T_{\text {end point }}$ is the temperature at the end of the $S$ curve. $T_{\text {mid point }}$ refers to the mid point between the beginning and the end of $S$ curve. The point $T_{\max }$ on the DTG curve shows the temperature at which the maximum rate of reaction occurs.

Another widely used characterization for fuels is the Proximate Analysis. The Proximate analysis for the samples measured for this Atlas was determined by ASTM methods. It gives the amount of material that volatilizes up to $950^{\circ} \mathrm{C}$ in an inert atmosphere, the fixed carbon and the ash content. Since moisture varies widely all values are on a dry basis.

We have included as Appendix $A$ a new large collection of both proximate and ultimate analyses collected from the literature with both experimental and calculated heats of combustion. A new formula relating the heat of combustion to the elemental (ultimate) 
analysis is given [Chaniwalla, 1992].

Almost all of the data are presented with the same scale coordinates so that the data for different samples can be compared. We have provided Figure 3, a blank graph, to enable the reader to trace one curve and compare it with any other by making a transparency copy of Fig. 3 and then tracing the curves.

Biomass is the generic name for agricultural, forestry and other naturally occurring organic materials and wastes when considered as a source of energy and chemicals. Though biomass occurs in innumerable forms, the principal components are cellulose, hemicellulose, lignin and extractives. Chapter 1 contains data on these components of biomass.

Chapter 2 contains TA data on the natural forms of woody, agricultural and aquatic biomass. Thermal analysis data on processed biomass such as paper, pellets, etc. is collected in Chapter 3. Municipal solid waste (MSW) and waste components such as plastics are arranged in Chapter 4. Solid fuels such as coal and lignite representative of fuels with high fixed carbon content are presented in Chapter 5 . Liquid fuels are brought together in Chapter 6.

In Chapter 7 the variations due to the plant age, growth conditions and anatomy are shown. As discussed previously, TG thermograms also known as TG fingerprints vary with the conditions of measurement. The effect of varying the experimental conditions are shown in Chapter 8. One set of standard conditions was used for most of the samples presented in this atlas (3-10 mg samples; heated at $10 \mathrm{C} / \mathrm{min}$. as discussed in Chapter 10 on experimental data), but the examples in this chapter make it possible to extrapolate these data to other conditions for most of the samples when used in conjunction with the kinetic equation given in Chapter 10.

Chapter 9 gives an overview of TG, DTG and DTA, techniques. Chapter 10 discusses how thermal analysis data obtained by TG and DTA respectively can be used to derive kinetic data and extrapolate to other conditions. The experimental apparatus and the conditions used for collecting the data in this atlas, along with the causes of errors during the data collection, form the basis of Chapter 11.

An initial scanning of the Atlas shows a great deal of similarity between the various thermograms. For instance, one is tempted to say about biomass that it starts to break down about $200^{\circ} \mathrm{C}$ and is essentially completely decomposed by $500^{\circ} \mathrm{C}$. For some purposes this generalization is sufficient. However, for other purposes a more exact knowledge of the temperatures at which mass or energy changes occur is absolutely necessary. We have taken the liberty of making a few observations in each chapter on particular features that we have observed. We hope the reader will look at the thermograms for the differences, rather than the similarities and find useful information. 
We present this ATLAS OF THERMAL DATA in the hopes that it will be useful for scientists who wish to understand more about the mechanism of pyrolysis and to engineers working in the field who need accurate data to design experiments and projects.

Siddhartha Gaur and Thomas B. Reed Chemical Engineering and Petroleum Refining Department Colorado School of Mines, Golden, Colorado, 80401 January, 1995 


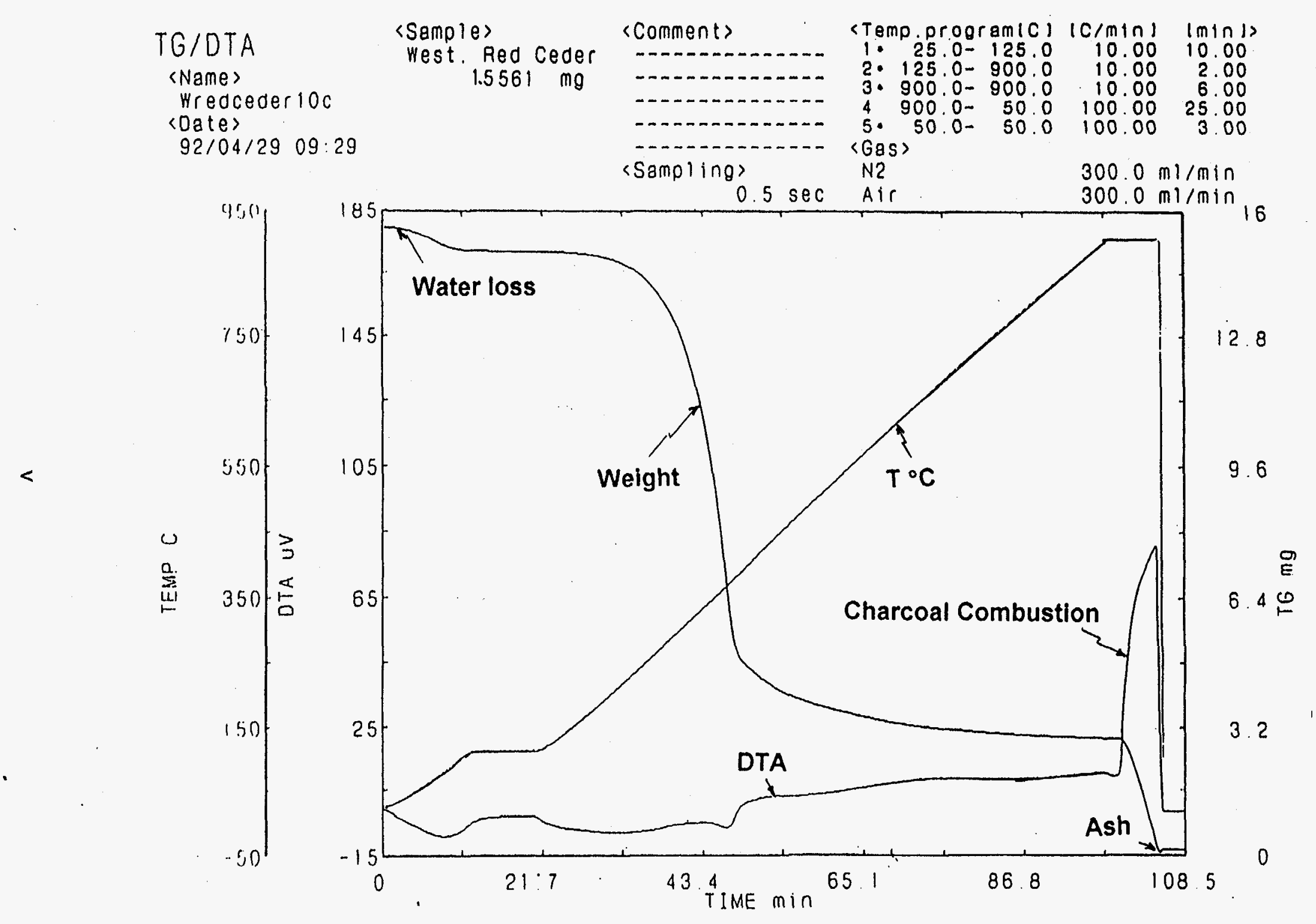

Figure 1. Typical experimental data on Western red cedar showing heating program, temperature (T), weight (TG) and differential temperature ((DTA) vs time, char combustion in air and final ash weight. 

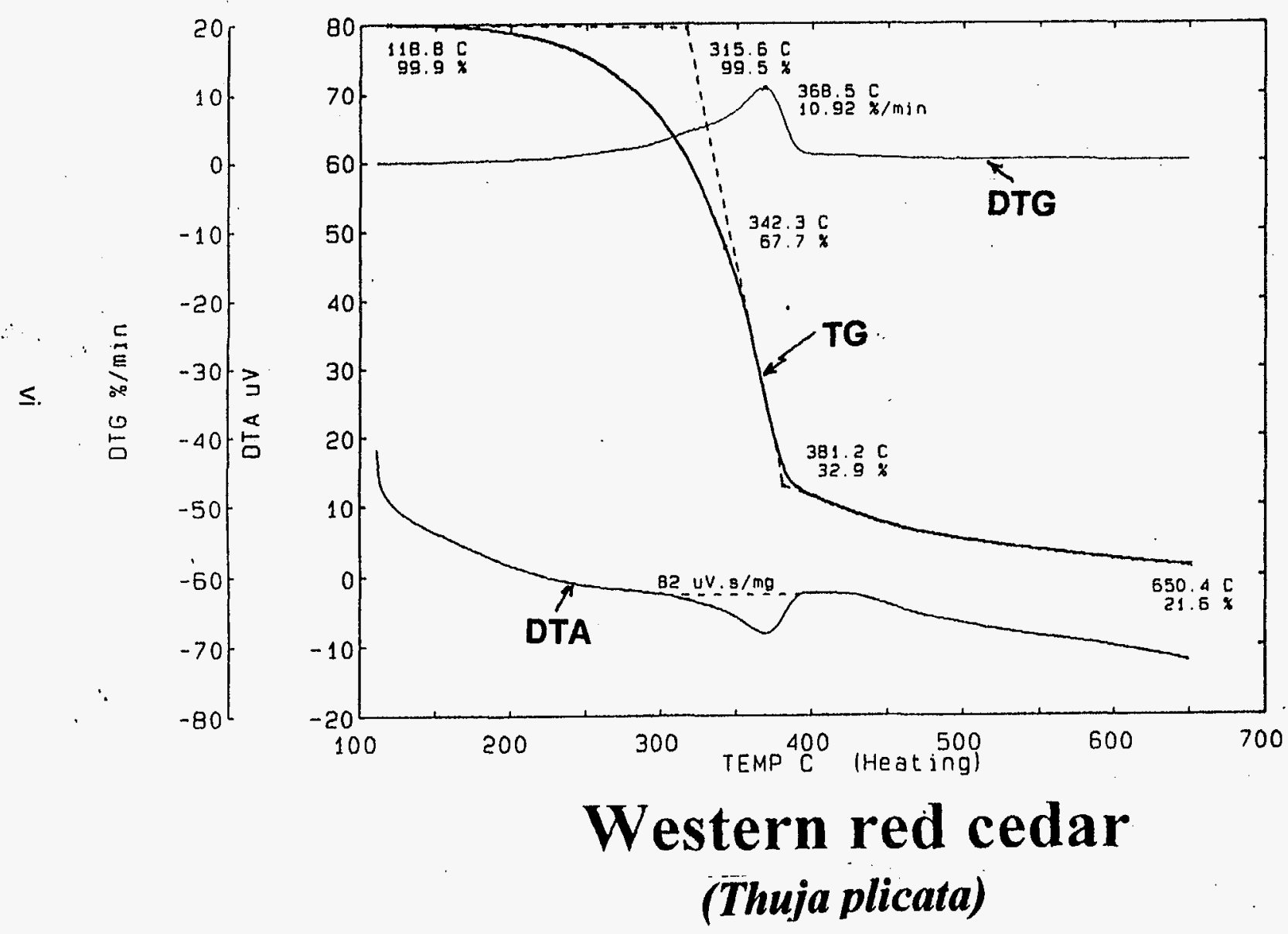

\begin{tabular}{|c|c|}
\hline 90 & $\begin{array}{l}\text { Western red cedar } \\
\text { (Tuja plicata) }\end{array}$ \\
\hline \multirow[b]{2}{*}{ BO } & Heating rate- ${ }^{\circ} \mathrm{C} / \mathrm{min}: 10$ \\
\hline & Sample weight-mg: 15.35 ; \\
\hline 70 & $T_{\text {initu| }}{ }^{-\mathrm{C}}: 118.8$ \\
\hline \multirow{2}{*}{60} & Volatilization \\
\hline & $T_{\text {tunn-polnt }}{ }^{\circ} \mathrm{C}: 315.6$ \\
\hline 50 & $T_{\text {mid. polm }}{ }^{\circ} \mathrm{C}: \quad 342.3$ \\
\hline \multirow{2}{*}{$\begin{array}{r}x \\
40 \Leftrightarrow\end{array}$} & $\mathrm{T}_{\text {end.poin }}{ }^{\circ} \mathrm{C}: 381.2$ \\
\hline & $T_{\text {max }}{ }^{\circ} \mathrm{C}: 368.5$ \\
\hline 30 & Rate $_{\max }-\mathrm{mg} / \mathrm{min}: 10.92$ \\
\hline \multirow{2}{*}{20} & $W_{t .} \%_{0 s 0} c^{-:}: 21.6$ \\
\hline & $\Delta \mathrm{H}_{\mathrm{vol}}-\mu \mathrm{v} \cdot \mathrm{sec} / \mathrm{mg}: 82$ \\
\hline 10 & Proximate analysis \\
\hline \multirow{5}{*}{0} & Volatiles $_{T_{\alpha \alpha}|-w| \%: 80.2}$ \\
\hline & Volatiles $_{s s 0} \mathrm{c} \cdot w \mathrm{t} \%: 78.4$ \\
\hline & Volatiles $_{s s 0-950} c^{-w t} \%: 1.8$ \\
\hline & Fixed carbon-wt $\%: 18.0$ \\
\hline & Ash-wt\%: 1.8 \\
\hline
\end{tabular}

Figure 2. Typical thermogram showing Weight Loss, (TG), the Derivative of Weight Loss, (DTG), and variation of heat production or absorption (DTA), vs Temeprature over the range $100^{\circ} \mathrm{C}-700^{\circ} \mathrm{C}$. Sidebar shows critical temperature points and proximate analysis results. 


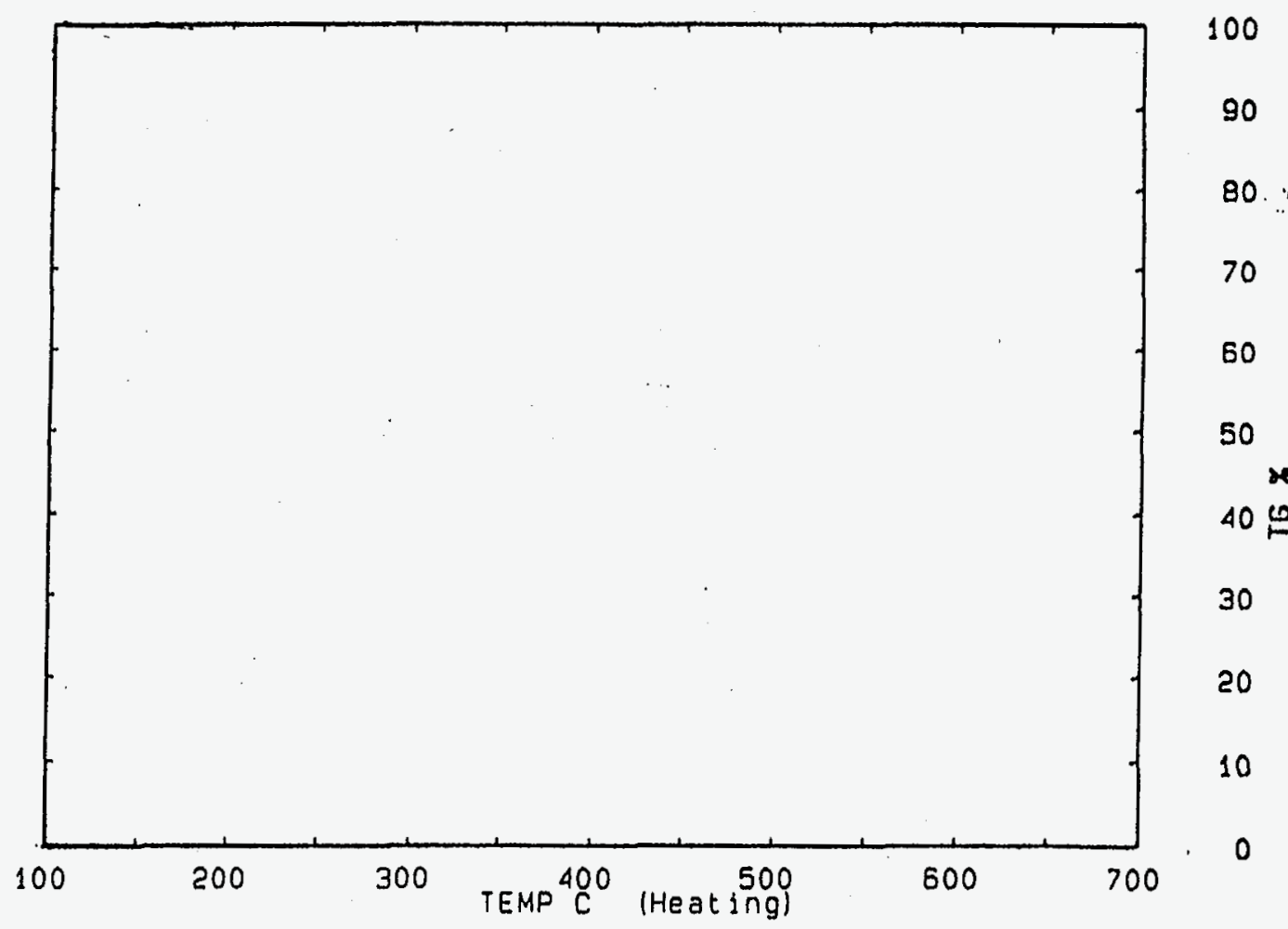

Figure 3. Blank Chart to permit Overlay Comparison of Different Thermograms. (Make transparency copies of this chart.) 


\section{CONTENTS}

Page

ABOUT THE ATLAS $\ldots \ldots \ldots \ldots \ldots \ldots \ldots \ldots \ldots \ldots \ldots \ldots \ldots \ldots \ldots \ldots$

ACKNOWLEDGMENTS $\ldots \ldots \ldots \ldots \ldots \ldots \ldots \ldots \ldots \ldots \ldots \ldots \ldots \ldots \ldots$

\section{CHAPTER 1 - COMPONENTS OF BIOMASS}

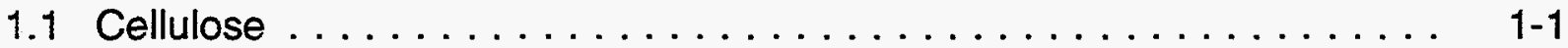

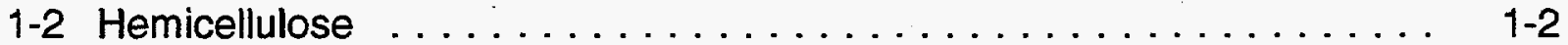

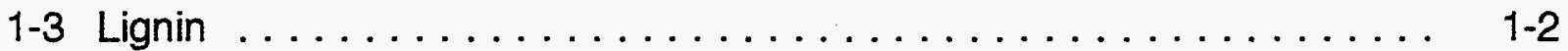

$1-4$ Extractives . . . . . .

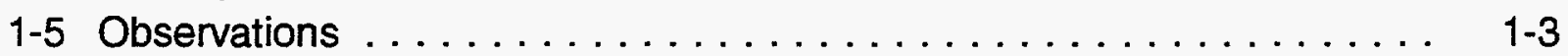

\section{THERMOGRAMS}

Cellulose

Cellulose (Avicel $₫$ PH 102) . . . . . . . . . . . . . . . . . . . 1-4

Cellulose (Baker analyzed) . . . . . . . . . . . . . . . . 1-5

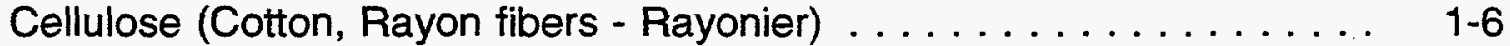

Cellulose, Cotton (Gossypium) . . . . . . . . . . . . . . 1-7

Cellulose from Bacteria (Acetobactor xylinum cellulose) $\ldots \ldots \ldots \ldots$ 1-8

Cellulose (Doped with $0.1 \% \mathrm{KOH}$ ) . . . . . . . . . . . . . . . . 1-9

Cellulose (Doped with $0.1 \% \mathrm{ZnCl}_{2}$ ) $\ldots \ldots \ldots \ldots \ldots \ldots \ldots \ldots \ldots$ 1-10

Hemicellulose

Hemicellulose, Acetylated xylan $\ldots \ldots \ldots \ldots \ldots \ldots \ldots \ldots \ldots$ 1-11

Lignin

Lignin-Steam exploded Aspen (Populus tremuloides) $\ldots \ldots \ldots \ldots \ldots$. . . .12

Lignin, Ball milled wood (Populus deltoides) . . . . . . . . . . . 1-13

Lignin, Brown rotten wood $(S$. pinus by $P$. placenta) $\ldots \ldots \ldots \ldots \ldots$ 1-14

Lignin, Kraft (Pinus) . . . . . . . . . . . . . . . . . . . . . 1-15

\section{Extractives}

Glucose (Baker) . . . . . . . . . . . . . . . . . . . . . . . 1-16

Wattle $\operatorname{tanin}($ Acacia sp.) . . . . . . . . . . . . . . . 1-17

\section{CHAPTER 2 - Natural Biomass}

2.1 Softwoods . . . . . . . . . . . . . . . .

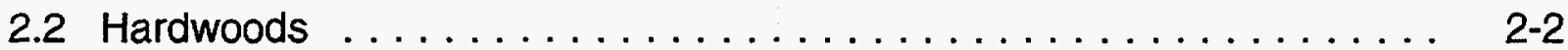

2.3 Agricultural Residues $\ldots \ldots \ldots \ldots \ldots \ldots \ldots \ldots \ldots \ldots \ldots . \ldots \ldots$ 2-2

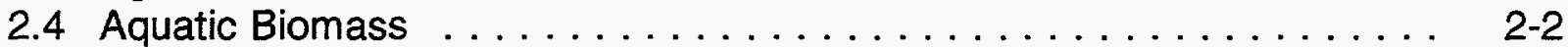

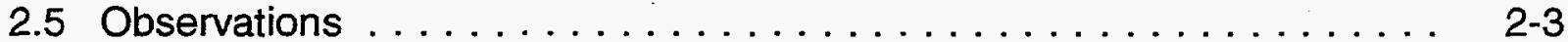

THERMOGRAMS

\section{Softwoods}

Eastern red cedar (Juniperus virginiana) $\ldots \ldots \ldots \ldots \ldots \ldots \ldots \ldots . \ldots .4$

Lodgepole pine (Pinus contorta) $\ldots \ldots \ldots \ldots \ldots \ldots \ldots \ldots \ldots \ldots . \ldots \ldots$

Monterey pine (Pinus radiata) $\ldots \ldots \ldots \ldots \ldots \ldots \ldots \ldots \ldots \ldots . \ldots \ldots$

Ponderosa pine (Pinus ponderosa) $\ldots \ldots \ldots \ldots \ldots \ldots \ldots \ldots \ldots$ 2-7

Ponderosa pine bark (Pinus ponderosa) $\ldots \ldots \ldots \ldots \ldots \ldots \ldots .2-8$

Redwood (Sequoia sempervirens) . . . . . . . . . . . . . . . 2-9

Slash pine (Pinus elliottii) $\ldots \ldots \ldots \ldots \ldots \ldots \ldots \ldots \ldots \ldots \ldots . . .2-10$ 
Sugar pine (Pinus lambertiana) . . . . . . . $\ldots \ldots \ldots \ldots \ldots$ 2-11

Trembling aspen (Populus tremuloides) . . . . . . . . . . . . . 2-12

Western hemlock (Tsuga heterophylla) $\ldots \ldots \ldots \ldots \ldots \ldots \ldots \ldots$ 2-13

Western red cedar (Thuja plicata) . . . . . . . . . . . . . 2-14

Hardwoods

Babool wood (Acacia arabica, India) . . . . . . . . . . . . . 2-15

Bass wood (Tilia americana) . . . . . . . . . . . . . . 2-16

Black locust (Robinia pseudoacacia) . . . . . . . . . . . . . 2-17

Black walnut (Juglans nigra) . . . . . . . . . . . . . . . 2-18

Eastern cottonwood (Populus deltoides) . . . . . . . . . . . . 2-19

Osage orange (Maclura pomifera) . . . . . . . . . . . . . 2-20

Red alder bark (Alnus rubra) . . . . . . . . . . . . . . . . 2-21

Red oak bark (Quercus rubra) . . . . . . . . . . . . . . . . . . . 2-22

Rubber wood (Hevea brasiliensis) . . . . . . . . . . . . . . 2-23

Agricultural Residues

Coconut shell (Cocos nucifera) $\ldots \ldots \ldots \ldots \ldots \ldots \ldots \ldots \ldots \ldots . .2-24$

Cotton Gossypiump) . . . . . . . . . . . . . . . . . . . . . . 2-25

Peach seed (Prunus persica) $\ldots \ldots \ldots \ldots \ldots \ldots \ldots \ldots \ldots \ldots \ldots . \ldots . . .2-26$

Peanut shell (Arachis hypogaea) . . . . . . . . . . . . . . . . . . . 2-27

Pistachio nut (Pistacia vera) . . . . . . . . . . . . . . . 2-28

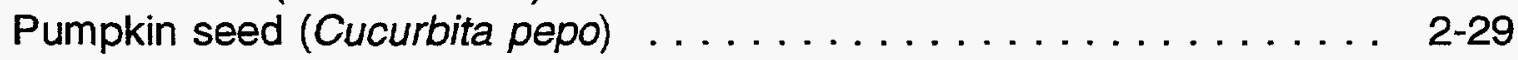

Rice hull (Oryza sativa) . . . . . . . . . . . . . . . . . . . . 2-30

Sericea lespedeza (Sericea lespedeza cuneata var. serala) . . . . . . 2 2-31

Sorghum (Sorghum bicolor) . . . . . . . . . . . . . . . . . 2-32

Spinach stem (Spinacea oleracera) . . . . . . . . . . . . 2-33

Sugar cane baggase Saccharum officinarum) $\ldots \ldots \ldots \ldots \ldots \ldots .2-34$

Sunflower stalk (Helianthus) . . . . . . . . . . . . . . . . 2-35

Switch grass (Panicum virgatum L.) . . . . . . . . . . . 2-36

Wheat Straw (Triticum aestivum, var Thunderbird) . . . . . . . 2-37

Auquatic Biomass

Eelgrass (Zostera marina) . . . . . . . . . . . . . . . . . . . 2-38

Giant kelp (Laminariaceae) . . . . . . . . . . . . . . . . . . 2-39

Sargassum weed (Sargassum bacciferum) $\ldots \ldots \ldots \ldots \ldots \ldots .2-40$

\section{CHAPTER 3 - PROCESSED BIOMASS}

3.1 Paper . . . . . . . . . . . . . .

3.2 Densified Biomass $\ldots \ldots \ldots \ldots \ldots \ldots \ldots \ldots \ldots \ldots \ldots \ldots \ldots$ 3-1

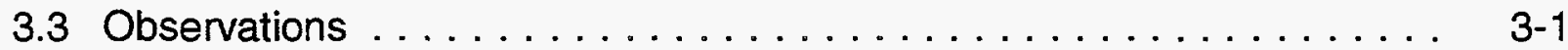

THERMOGRAMS

Paper, Newsprint . . . . . . . . . . . . . . . . . . . 3-2

Paper, Kimwipe tissue $\ldots \ldots \ldots \ldots \ldots \ldots \ldots \ldots \ldots \ldots \ldots \ldots$ 3-3

Paper, $25 \%$ Cotton $\operatorname{mix} \ldots \ldots \ldots \ldots \ldots \ldots \ldots \ldots \ldots \ldots \ldots . \ldots \ldots$ 3-4

Cardboard, Corrugated sheet $\ldots \ldots \ldots \ldots \ldots \ldots \ldots \ldots \ldots \ldots$ 3-5

Cardboard, Liner board . . . . . . . . . . . . . . . . . . . . . . 3-6

4.1 Municipal Solid Waste $\ldots \ldots \ldots \ldots \ldots \ldots \ldots \ldots \ldots$. 4 .

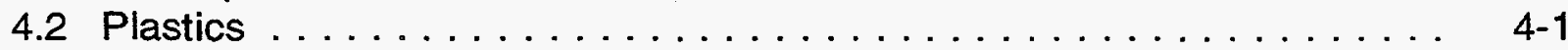


4-3 Observations $\ldots \ldots \ldots \ldots \ldots \ldots \ldots \ldots \ldots \ldots \ldots \ldots \ldots \ldots$ 4-3

THERMOGRAMS

Ecofuel-2 . . . . . . . . . . . . . . . . . . . . . . . . . . 4-2

Refuse derived fuel (Teledyne) . . . . . . . . . . . . . . 4-3

Refuse derived fuel (Thief river falls) $\ldots \ldots \ldots \ldots \ldots \ldots \ldots \ldots .44$

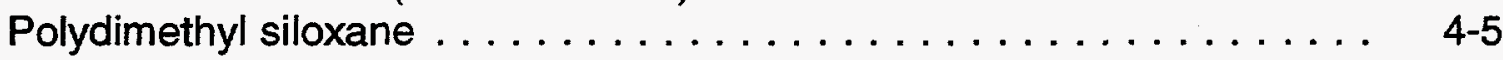

Polyethylene ......................... 4-6

Polyethylene glycol . . . . . . . . . . . . . . . . . . . 4-7

Polyhydroxy benzoic acid $\ldots \ldots \ldots \ldots \ldots \ldots \ldots \ldots \ldots \ldots$ 4.8

Polymethyl methacrylate $\ldots \ldots \ldots \ldots \ldots \ldots \ldots \ldots \ldots \ldots$ 4 .9.

Polystrene ... . . . . . . . . . . . . . . . . . . . . . . 4-10

Polytetraflouroethylene (Teflon) . . . . . . . . . . . . . . . . 4-11

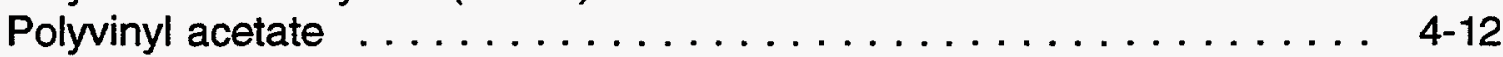

Polyvinyl chloride $\ldots \ldots \ldots \ldots \ldots \ldots \ldots \ldots \ldots \ldots \ldots \ldots \ldots \ldots \ldots \ldots$

\section{CHAPTER 5 - HIGH CARBON SOLID FUELS}

5.1 Charcoal .............................. $5-1$

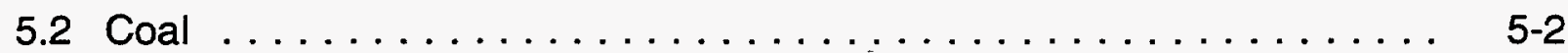

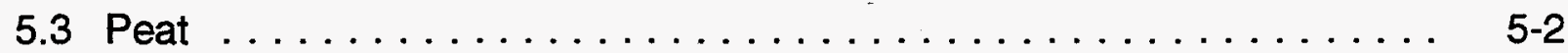

\section{THERMOGRAMS}

Switchgrass char (Panicum virgatum L.) $\ldots \ldots \ldots \ldots \ldots \ldots \ldots$ 5-3

Coal, Blind Canyon . . . . . . . . . . . . . . . . . . 5-4

Coal, Buelah zap lignite (North Dakota) . . . . . . . . . . . . . 5-5

Coal, Illinois \#6 ........................... 5-6

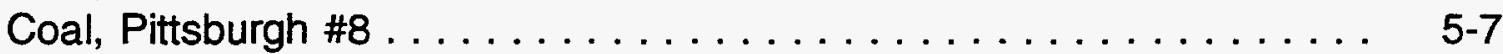

Coal, Wyodak bituminous . . . . . . . . . . . . . . . .

Peat, (Pahokee Point, Fla) . . . . . . . . . . . . . . . . . . . 5- 5

Peat, (Steam exploded) $\ldots \ldots \ldots \ldots \ldots \ldots \ldots \ldots \ldots \ldots \ldots \ldots$. $5-10$

CHAPTER 6 - LIQUID FUELS

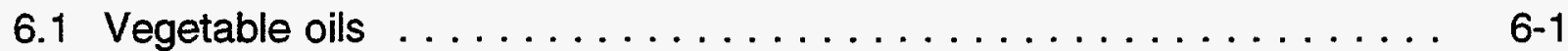

6.2 Biodiesel . . . . . . . . . . . .

6.3 Pyrolysis Oils . . . . . . . . . . . .

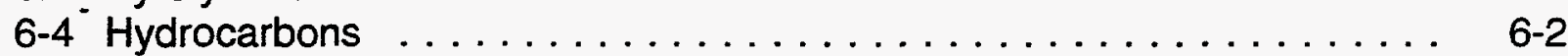

THERMOGRAMS

Corn oil . . . . . . . . . . . . . . . . . . . . . . . . 6-3

Biodiesel (Corn oil-methl ester) . . . . . . . . . . . . . . . . . . 6-4

Waste vegetable oil (Methyl ester) . . . . . . . . . . . . . . 6 6-5

Glycerol . . . . . . . . . . . . . . . . . . . . . . . . . . 6-6

Red Oak Pyrolysis oil (Quercus rubra) . . . . . . . . . . . . . . . . 6-7

Rubber tire pyrolysis oil . . . . . . . . . . . . . . . . . . 6 6-8

Switchgrass pyrolysis oil (Panicum virgatum L.) . . . . . . . . . 6-9

Diesel fuel . . . . . . . . . . . . . . . . . . . . . . . . . 6-10

Paraffin (Carbowax) ..................... 6.11

CHAPTER 7 - VARIATIONS IN BIOMASS

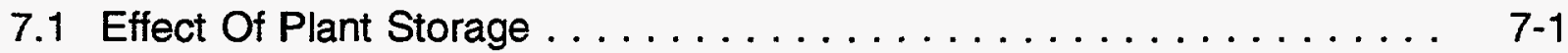

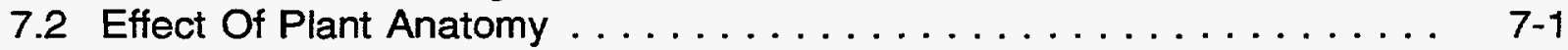




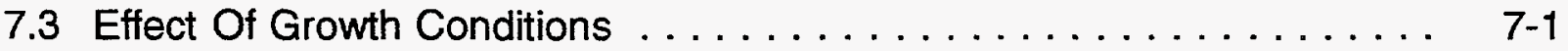

\section{THERMOGRAMS}

Cotton, dryland (Gossypium) . . . . . . . . . . . . . . 7-2

Cotton, Irrigated (Gossypium) . . . . . . . . . . . . . . . 7-3

Effect of growth conditions (Dryland and irrigated cotton) . . . . . . . 7-4

Eastern cottonwood stored for zero weeks (Populus deltoides) . . . . . . 7-5

Eastern cottonwood stored for 26 weeks (Populus deltoides) . . . . . . 7-6

Sericea lespedeza stored for zero weeks (Lespedez cuneata) . . . . . . 7-7

Sericea lespedeza stored for 26 weeks (Lespedeza cuneata) . . . . . . 7 7-8

Sorghum stored for zero weeks (Sorghum bicolor) . . . . . . . . . . . 7-9

Sorghum stored for 26 weeks (Sorghhum bicolor) . . . . . . . . . 7-10

Spinach stem xylem and phloem (Spinacea oleracea) . . . . . . . . 7-11

Spinach stem cell chloroplast (Spinacea oleracea) . . . . . . . . . . 7-12

Spinach cell cytoplasmic liquid (Spinacea oleracea) . . . . . . . . . . . 7-13

Sugar cane baggase stored for zero weeks (Saccharum officinarum) . . . 7-14

Sugar cane baggase stored for 26 weeks (Saccharum officinarum) . . . 7-15

Switchgrass stored for zero weeks (Panicum virgatum L.) . . . . . . 7-16

Switchgrass stored for 26 weeks (Panicum virgatum L.) . . . . . . . 7-17

Brown rotted wood (S. pinus by $P$. placenta) . . . . . . . . . . . . 7-18

White rotted wood (White spruce by Ganoderma applanatum) . . . . . 7-19

\section{CHAPTER 8 - EFFECT OF OPERATING CONDITIONS}

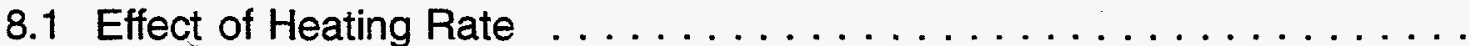

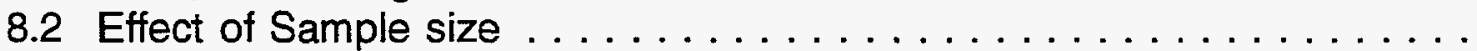

8.3 TG additivity

\section{THERMOGRAMS}

Effect of heating rate (Western red cedar)

Effect of heating rate (Pelletized Baker Cellulose)

Effect of particle size

Biomass components additivity (Populus deltoides)

\section{CHAPTER 9 - OVERVIEW OF THERMAL ANALYSIS METHODS}

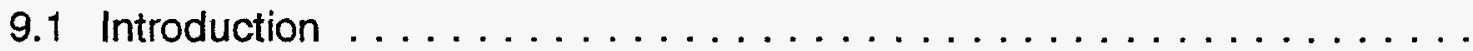

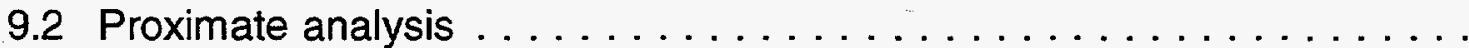

9.3 Ultimate analysis

9.4 Thermogravimetry and Differential thermogravimetry $\ldots \ldots \ldots \ldots$

9.5 Differential thermal analysis and Differential scanning calorimetry

9.5.1 Applications of differential thermal analysis

9.6 ICTA recommendations

9.7 Manufacturers

\section{CHAPTER 10 - DERIVING KINETIC DATA FROM THERMAL ANALYSIS}

10.1 Introduction $\ldots \ldots \ldots \ldots \ldots \ldots \ldots \ldots \ldots \ldots \ldots \ldots$ 10-1

10.2 Determination of kinetic parameters using thermogravimetric data . . . . 10-2

10.2.1 Coats and Redfern method . . . . . . . . . . . . . 10-2

10.2.2 Gyulai and Greenhow method . . . . . . . . . . . . . 10-3

10.2.3 Doyle's method . . . . . . . . . . . . . . . . . . . . . . . 10 10-5 
10.2.4 Zsako method . . . . . . . . . . . . . . . . . . 10-6

10.2.5 Satava and Skvara method . . . . . . . . . . . . . 10-7

10.2.6 Freeman and Carroll method . . . . . . . . . . . . . . . . 10-7

10.2.7 Ingraham and Marier method . . . . . . . . . . . . . . . . 10-8

10.2.8 Vachuska and Voboril method . . . . . . . . . . . . 10-8

10.2.9 Varhegyi's integral solution $\ldots \ldots \ldots \ldots \ldots \ldots \ldots \ldots \ldots$ 10-9

10.2.10 Gaur and Reed method . . . . . . . . . . . . . . . 10-10

10.3 Determination of kinetic parameters using differential thermal

analysis data . . . . . . . . . . . . . . . . . . . . 10-12

10.3.1 Other effects in understanding DTA data $\ldots \ldots \ldots \ldots \ldots \ldots 10-13$

\section{CHAPTER 11 - EXPERIMENTAL APPARATUS AND DATA COLLECTION}

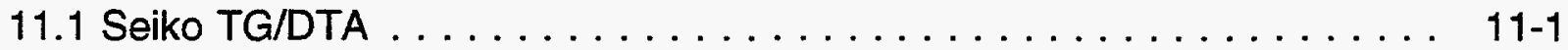

11.2 Procedure for data collection $\ldots \ldots \ldots \ldots \ldots \ldots \ldots \ldots \ldots \ldots$ 11-2

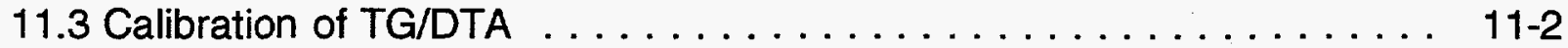

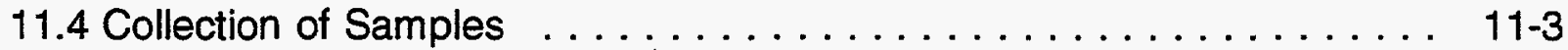

11.5 Errors in thermal analysis . . . . . . . . . . . . . . 11-3

11.5.1 The presence of temperature gradients in the sample . . . . 11-3

11.5.2 Use of an excessive heating rate $\ldots \ldots \ldots \ldots \ldots \ldots \ldots . \ldots \ldots$

11.5.3 The existence of a partial pressure of the product gases over the sample . . . . . . . . . . . . . $\ldots \ldots \ldots$ 11-4

11.5.4 The effect of impurities and mineral content . . . . . . . 11-4

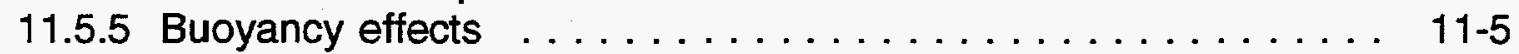

\section{REFERENCES}

INDEX

APPENDIX A: PROXIMATE, ULTIMATE AND $\triangle H_{C}$ DATA ON SELECTED FUELS APPENDIX B: SOURCES OF SAMPLES 


\section{Acknowledgments}

During the preparation of this Atlas help was provided by many people. We wish to thank Thomas Milne, Robert Evans, Foster Agblevor, Estaban Chornet, Ralph Overend and Jim Diebold from the National Renewable Energy Laboratory for participating in all stages of preparation of this Atlas.

We are indebted to Professor Robert M. Baldwin, Head of the Chemical Engineering and Petroleum Refining Department and Professor Kent Voorhees, Chemistry department, from the Colorado School of Mines, for allowing us to use the equipment and facilities. Professor Baldwin provided the needed encouragement to the project. We thank Mr. James Adams for conducting some of the test runs.

We are indebted to Professor Herbert Schroeder at the Colorado State University, the Paper Research Institute, Mr. Terry Highly of the U. S. Forest Products Laboratory in Madison Wisconsin, and the Marine Research Institute for supplying samples.

Finally, we thank our wives, Vibha Bansal and Vivian Reed, for being supportive to us during this work. 


\section{Chapter 1}

\section{COMPONENTS OF BIOMASS}

The word biomass was coined in the early 1970 s to refer to all living matter, but particularly with respect to use as a source of energy and fuel. It includes forest and agricultural species and even animal by-products such as manure and fats. It can refer to biomass grown specifically for energy, to biomass residues and even to municipal waste. Plants are composed primarily of cellulose, hemicellulose and lignin plus various particular "extractives (such as tannin) as shown in Table 1.1. These then constitute the majority of the thermal data in this section.

\section{Table 1.1 Composition of Typical Biomass plants}

$\begin{array}{lcclc}\text { COMMON NAME } & \text { CELLULOSE } & \text { HEMICELLULOSE } & \text { LUGNIN } & \text { OTHER } \\ \text { Softwoods } & 42.2 & 28 & 25-35 & \text { ca 0-1 } \\ \text { Hardwoods } & 42.2 & 38 & 15-20 & \text { ca 0-1 } \\ \text { Flax } & 71.2 & 18.6 & 2.2 & 8.0 \\ \text { Jute } & 71.6 & 13.3 & 13.1 & 2.0 \\ \text { Hemp } & 74.4 & 17.9 & 3.7 & 4.0 \\ \text { Ramie } & 76.2 & 14.6 & 0.7 & 8.5 \\ \text { Sisal } & 73.0 & 13.0 & 14.0 & 0.0 \\ \text { Abaca } & 70.2 & 21.8 & 5.7 & 2.3 \\ \text { Cotton, crude } & 95.3 & 0.0 & 0.0 & 4.7 \\ \text { Cotton, Purified } & 99.9 & 0.0 & 0.0 & 0.1\end{array}$

Carbohydrate is the generic name for cellulose, starch, inulin, sugars, chitin and other products of vegetable and animal metabolism [Kirk, 1983]. The name signifies hydrated carbon and comes from the fact that the formulas have the approximate ratio of $\mathrm{CH}_{2} \mathrm{O}$. Sugars represent available energy and chemicals in plants; starch and inulin are long term energy storage (insoluble) carbohydrates; cellulose and chitin are structural carbohydrates. The large carbohydrates (polysaccharides) are polymers of 5 and 6 carbon sugars, and in many cases can be hydrolysed to form the sugars.

\subsection{Cellulose}

Cellulose is by far the most common component of biomass and constitutes $30-100 \%$ of all or parts of plants as shown in Table 1.1. It is synthesized by marine and terrestrial plants but also by a few bacteria, animals (tunicates) and fungi. It forms the major part of the cell 
walls of all plants and is practically pure in the fibers of cotton, flax, jute and ramie [Kirk, 1983].

The cellulose molecule is a polymer of d-glucose of typically 1000 units (wood) up to 3,000 units (cotton). Regions of high crystalinity alternate with amorphous cellulose, forming elementary fibrils typically $3.5 \mathrm{~nm}$ in width and indefinite length.

The cellulose molecule has been studied more than any other component of biomass, and is relatively constant in structure compared to the other components. Therefore, there is a disproportionate amount of data in this Atlas on cellulose.

Cellulose can be converted back to glucose by acid or enzymatic hydrolysis, a process of interest in the production of ethanol from biomass and animal feeds [Kirk, 1983]. Essentially pure cellulose occurs in cotton and other plants. About $5 \times 10^{11}$ tons is biosynthesized in nature each year. It is commercially extracted from wood, primarily softwoods with long fibers, in the manufacture of paper [Weber, 1982] By far the largest use of cellulose, $45 X$ $10^{6}$ tons/yr., is in the paper industry, while 400,000 t/yr. is used for textile fibers.

\subsection{Hemicellulose}

Cellulose is a linear single compound, whereas, hemicelluloses are assembled from the fiveand six-membered sugars $d$-xylose, d-glucose, d-mannose, d-glactose, l-arabinose, dglucuronic acid, and many others [Kirk, 1983; Graboski 1981]. Hemicelluloses are composed of fewer monomers than cellulose, typically 300 and are often branched and may be attached to other functional units, particularly acetyl units.

Hemicellulose generally constitutes $12 \%-40 \%$ of most biomass [Table 1.1]. There are probably as many hemicelluloses as plant species, xylan is the most abundant of the hemicelluloses and is a polymer of the pentose d-xylose, $\left(\mathrm{C}_{5} \mathrm{H}_{10} \mathrm{O}_{5}\right)$.

\subsection{Lignin}

Lignin constitutes $4-35 \%$ of most biomass and is the principal non-carbohydrate fraction. It is a three-dimensional polymer of sinapyl, coniferyl and coumaryl alcohols based primarily on the phenylpropane structure. It is deposited in an amorphous state surrounding the cellulose fibers in wood and is bound by ether bonds directly to the cellulose and has no exact structure. Approximately $30 \times 10^{6}$ tons/yr. of lignin is extracted from wood. Much of it is burned, but some could be available for synthetic fuels [Kirk, 1983; Graboski, 1981].

\subsection{Extractives}

In addition to the above universal components, most biomass contains specific extractives such as terpenes (in coniferous trees, dessert plants, skins of fruits etc.) tannins (from oak, acacia etc.) oils, fragrances etc. These extractives may evaporate on storage or heating at relatively low temperatures and so typically do not show up in the thermograms in this Atlas. 


\subsection{Observations}

The thermogram of native and refined cellulose shows a very sharply defined decomposition over a range of about $30^{\circ} \mathrm{C}$, typically with a midpoint between $320^{\circ} \mathrm{C}$ and $380^{\circ} \mathrm{C}$. There is usually very little char remaining. An exception is the acid catalyst, $\mathrm{ZnCl}_{2}$, a fire retardent, which drops the midpoint to $270^{\circ} \mathrm{C}$ and leaves over $30 \%$ char at $500^{\circ} \mathrm{C}$.

The lignin decompositions extend over a range of over $100^{\circ} \mathrm{C}$ with a midpoints between $300^{\circ} \mathrm{C}$ and $400^{\circ} \mathrm{C}$ and leaves much more char. The range is so broad that it is not possible to define an energy for the reaction. The hemicellulose xylan decomposes at the lowest temperature, $275^{\circ} \mathrm{C}$. 

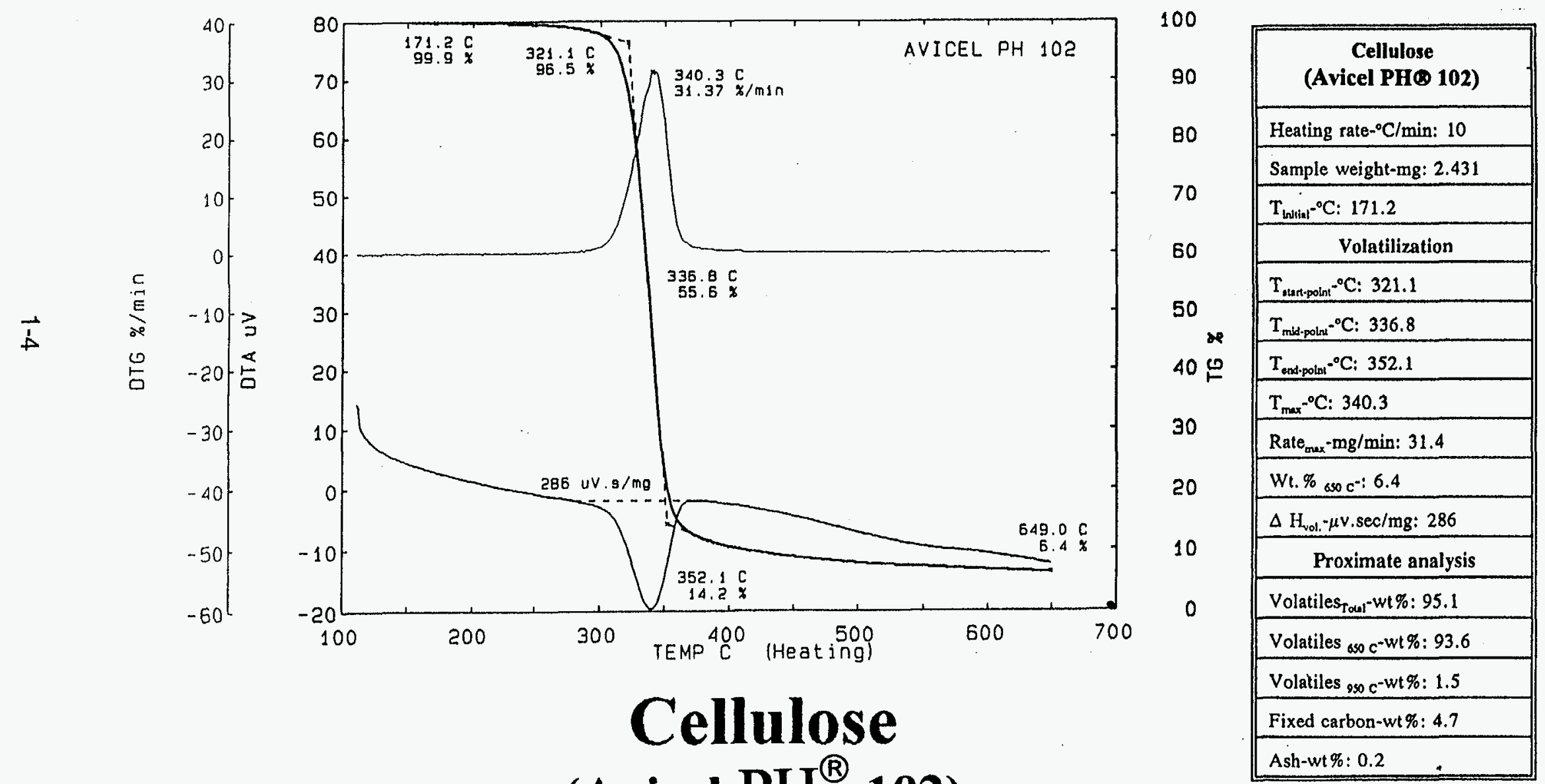

\section{(Avicel $\mathrm{PH}^{\circledR}$ 102)}

Cellulose 

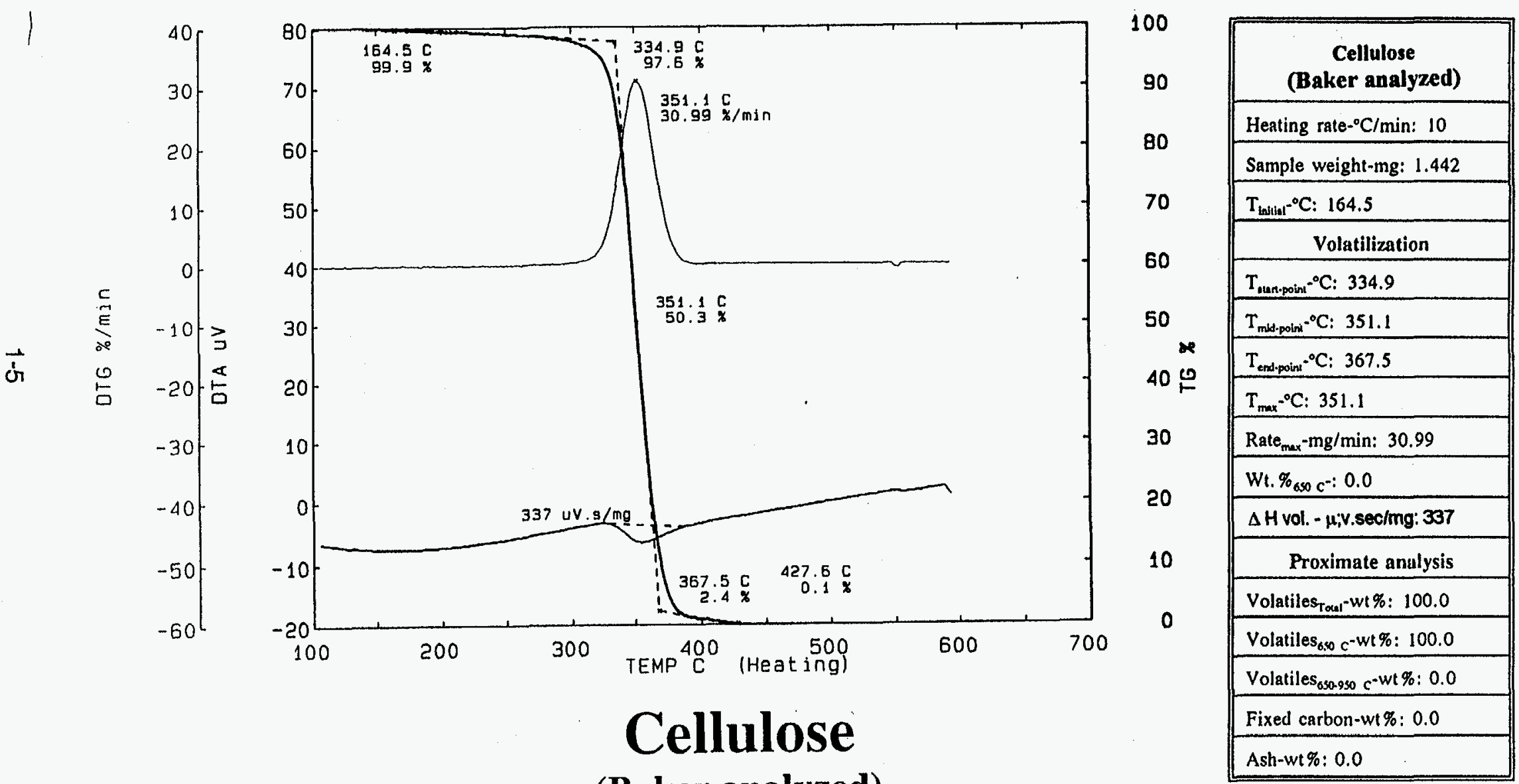


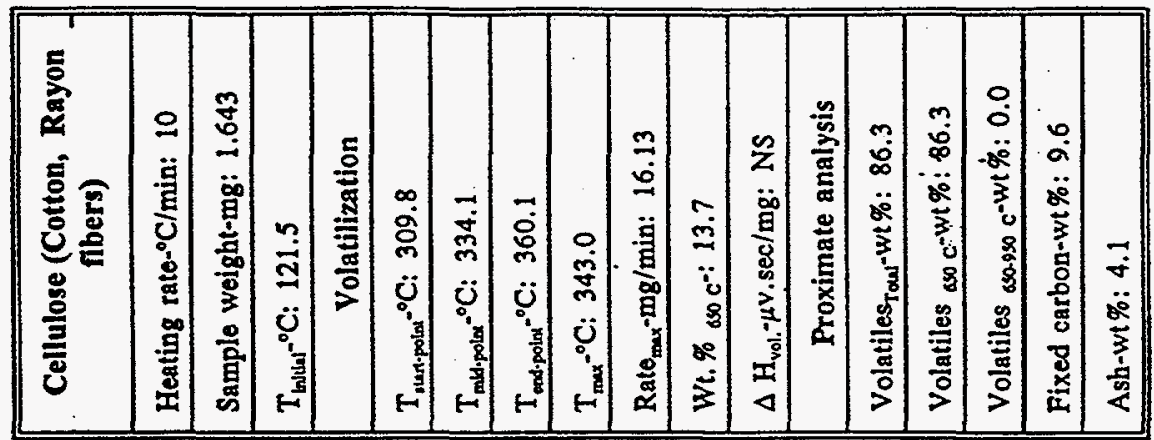

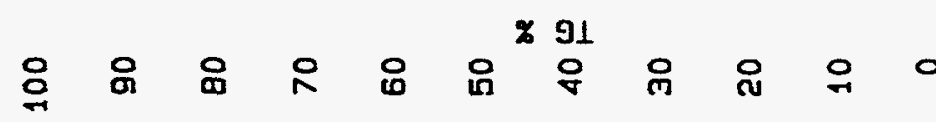
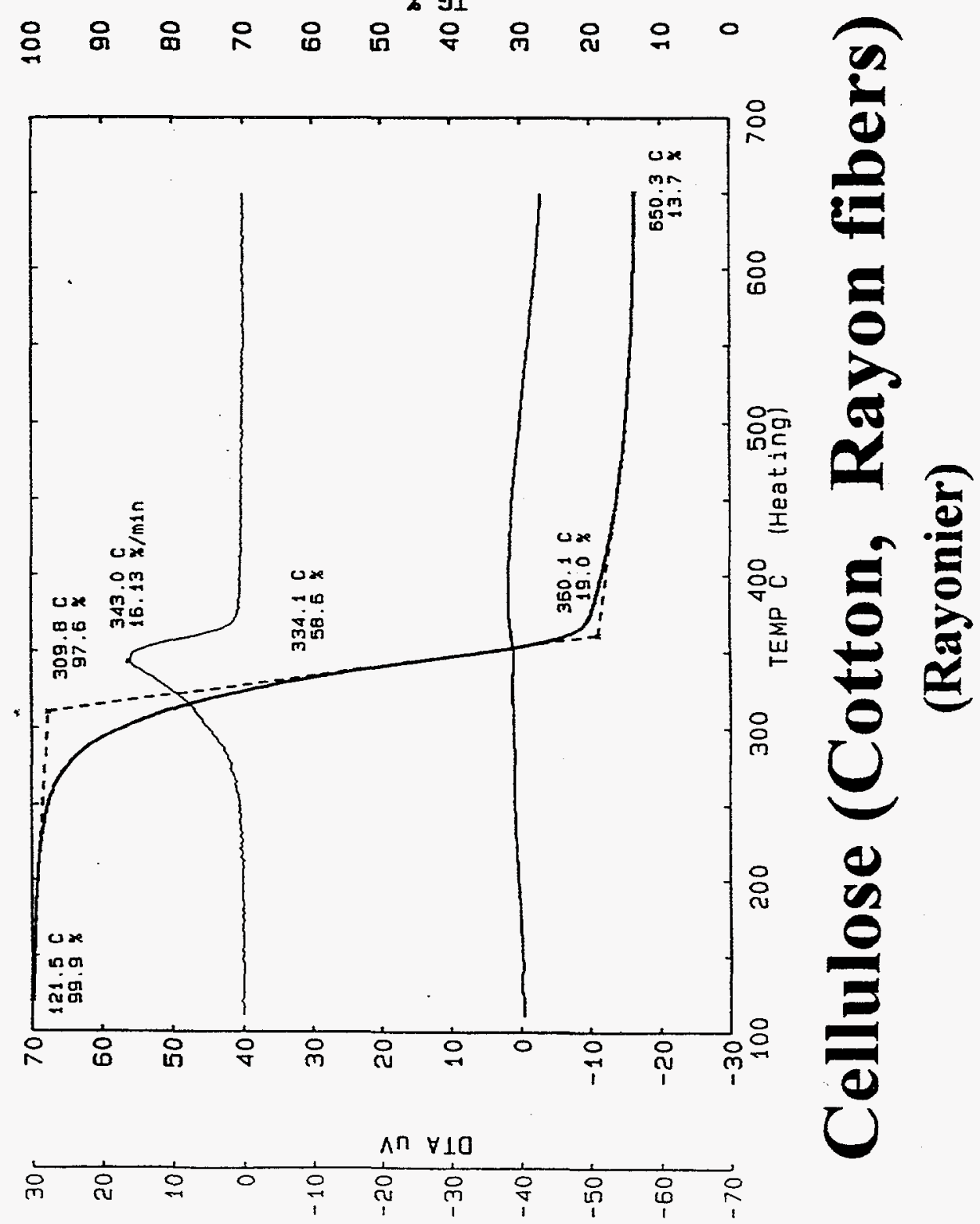

$u[\uplus / \% 910$ 


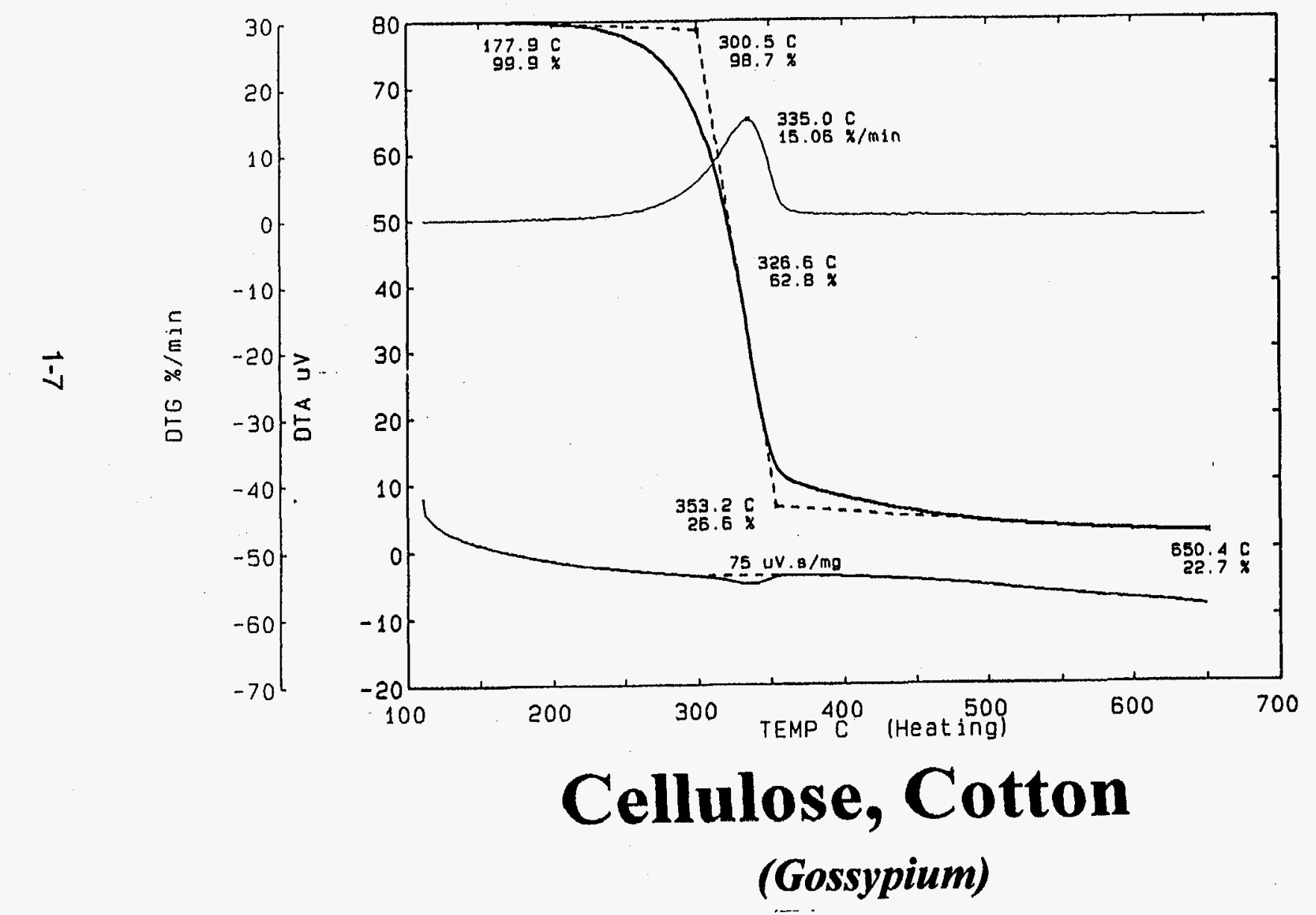

\begin{tabular}{|c|c|}
\hline \multicolumn{2}{|l|}{100} \\
\hline 90 & $\begin{array}{l}\text { Cellulose, Cotton } \\
\text { (Gossypium) }\end{array}$ \\
\hline \multirow{2}{*}{80} & \\
\hline & Heating rate. ${ }^{\circ} \mathrm{C} / \mathrm{min}: 10$ \\
\hline 70 & Sample weight-mg: 3.090 \\
\hline \multirow{2}{*}{60} & $\mathrm{~T}_{\text {Lerlital }}{ }^{-\mathrm{C}:}: 177.9$ \\
\hline & Volatilization \\
\hline \multirow{3}{*}{$\begin{array}{l}50 \\
40 \stackrel{0}{\circ}\end{array}$} & $T_{\text {oun.polut }}{ }^{-0} \mathrm{C}: 300.5$ \\
\hline & $T_{\text {mbl-posiat }}{ }^{-} \mathrm{C}: 326.6$ \\
\hline & $T_{\text {end-plotex }}{ }^{\circ} \mathrm{C}: \quad 353.2$ \\
\hline 30 & $\mathrm{~T}_{\max }{ }^{\circ} \mathrm{C}: 335.0$ \\
\hline \multirow{2}{*}{$20^{\circ}$} & Rate $_{\max }-\mathrm{mg} / \mathrm{min}: 15.06$ \\
\hline & Wt. \% osso $c^{-:}: 22.7$ \\
\hline \multirow[t]{2}{*}{10} & $\Delta \mathrm{H}_{\mathrm{vol}}-\mu \mathrm{v}, \mathrm{sec} / \mathrm{mg}: 75$ \\
\hline & Proximate analysis \\
\hline \multirow{5}{*}{0} & Volatiles $_{\text {Toul }}-w t \%: 79.4$ \\
\hline & Volatiles ${ }_{\text {sso }} c^{-w 1} \%: 77.3$ \\
\hline & Volatiles ${ }_{\text {ssa. }} 50 c^{-w t} \%: 2.1$ \\
\hline & Fixed carbon-wt\%: 13.4 \\
\hline & Ash-wt\%: 7.2 \\
\hline
\end{tabular}




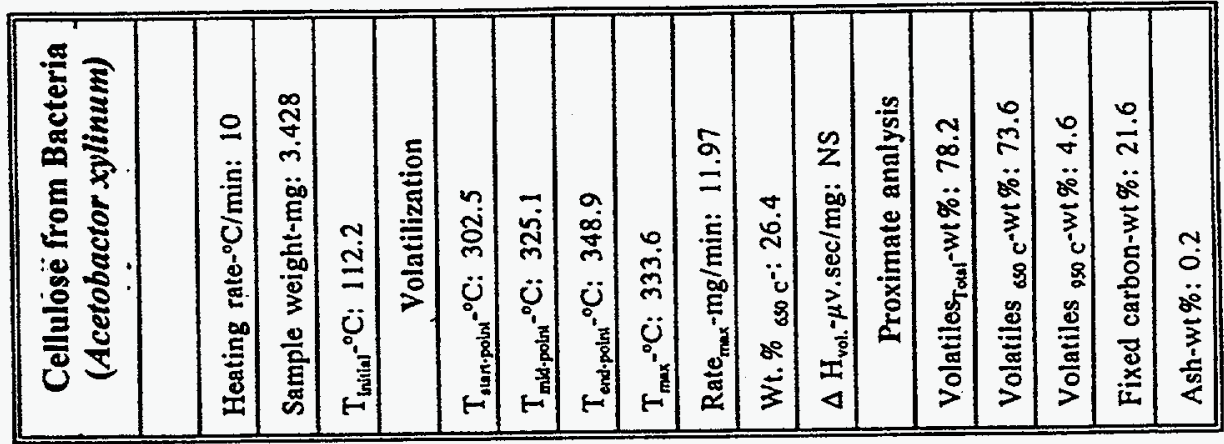

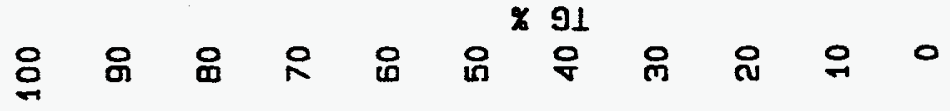

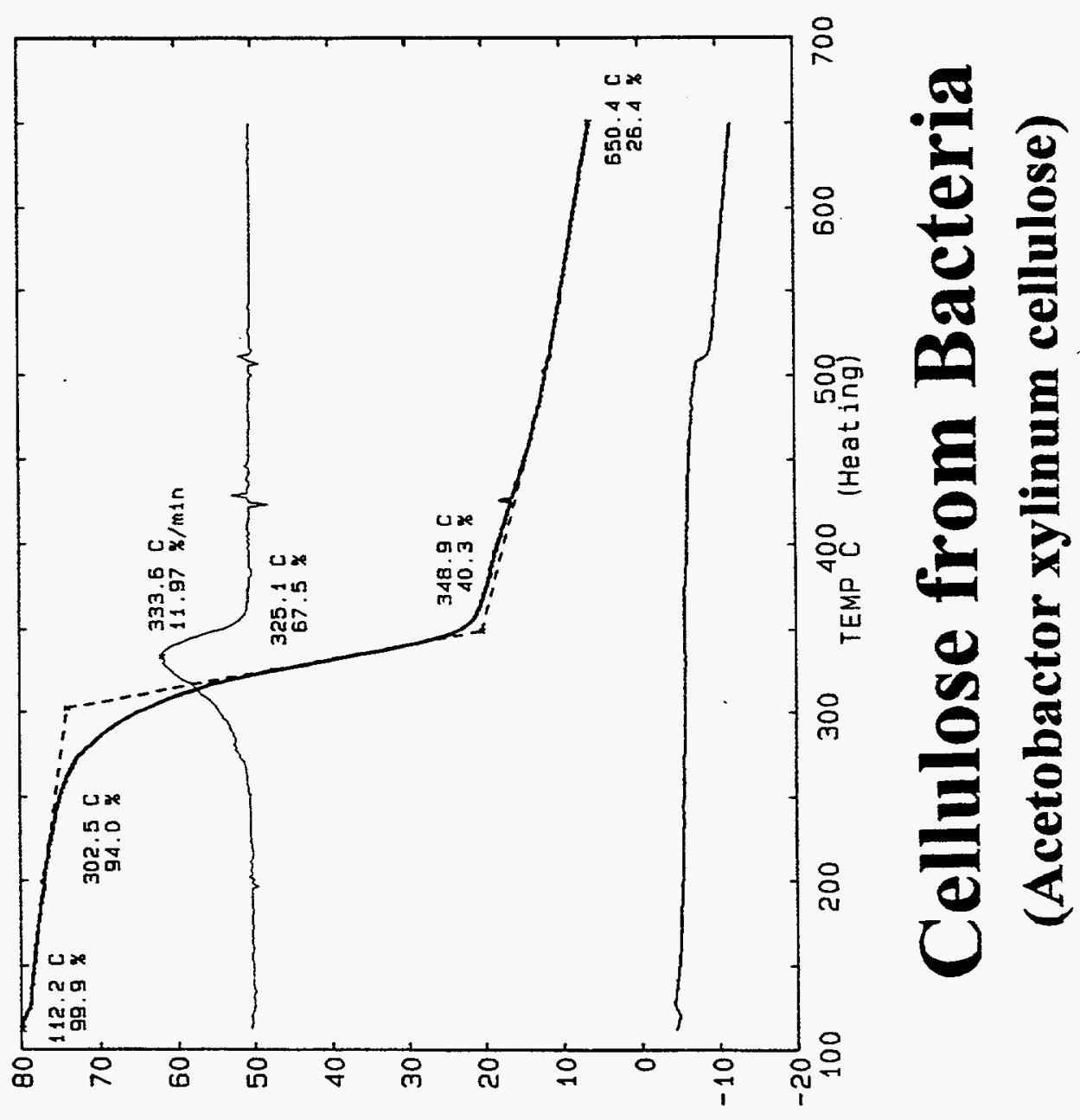

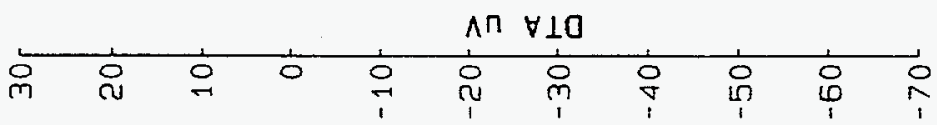

Uโบ/\% 910 


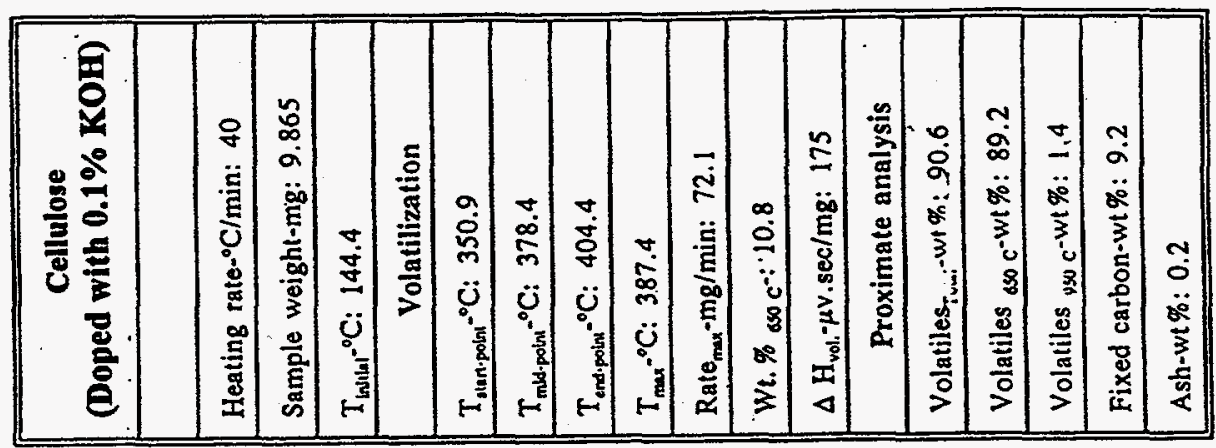

x 91

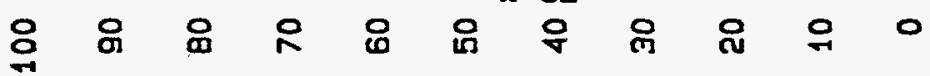

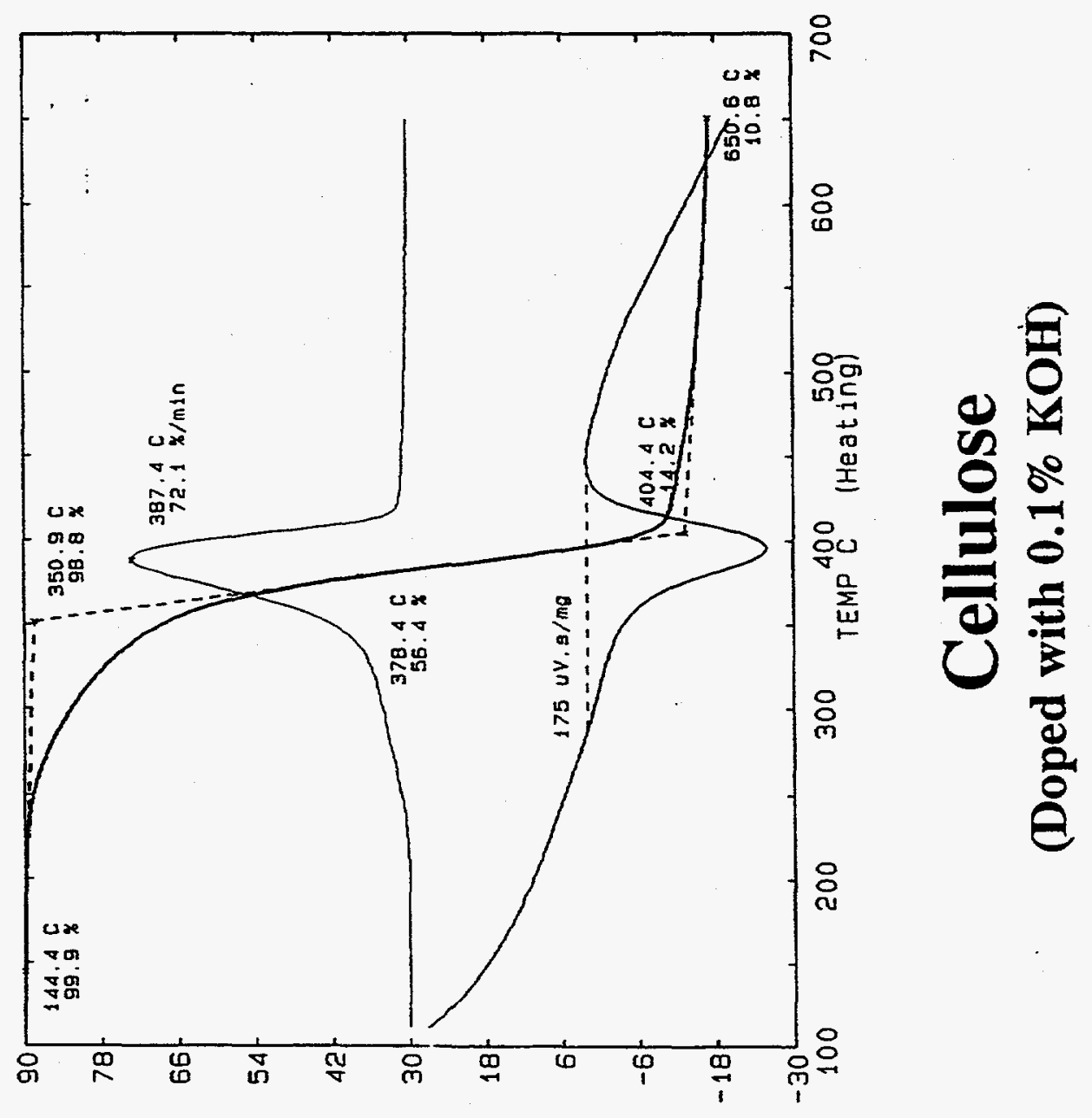

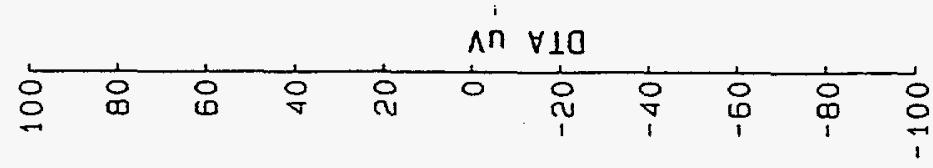

$u\lceil\omega / \% .910$ 

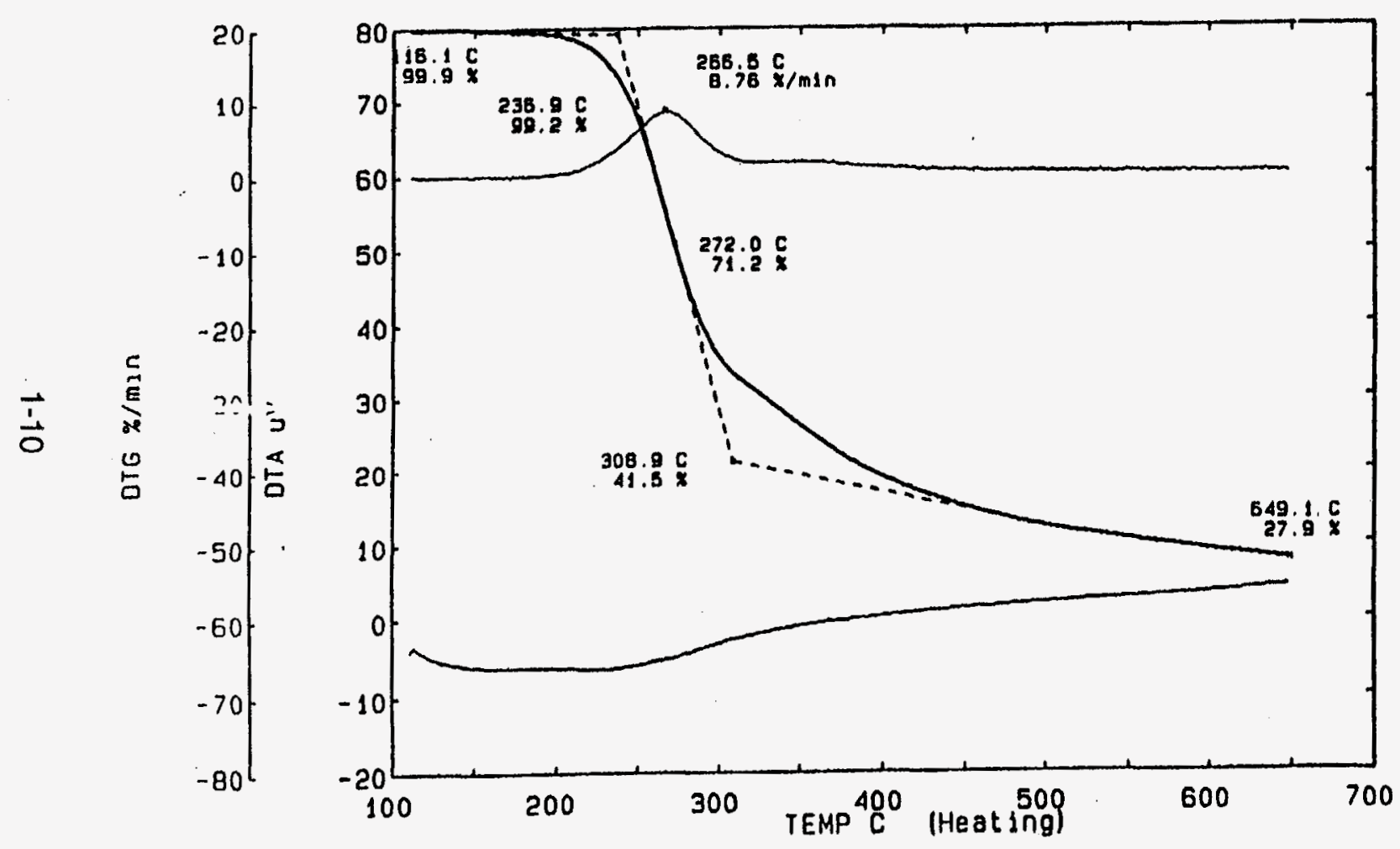

100

90

80

70

60

$40 \Leftrightarrow$

30

20

10

0

\section{Cellulose}

(Doped with $0.1 \% \mathrm{ZnCl}_{2}$ )

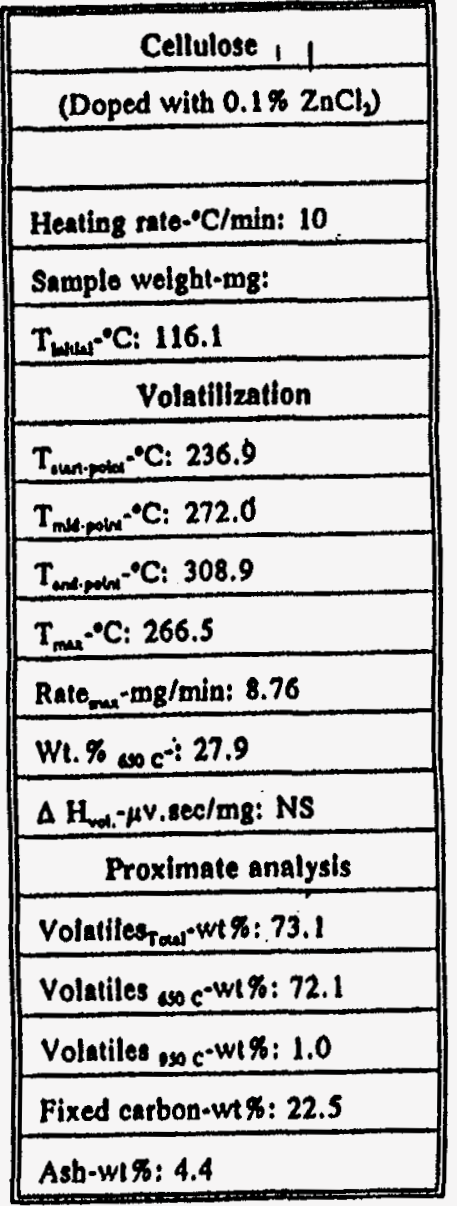



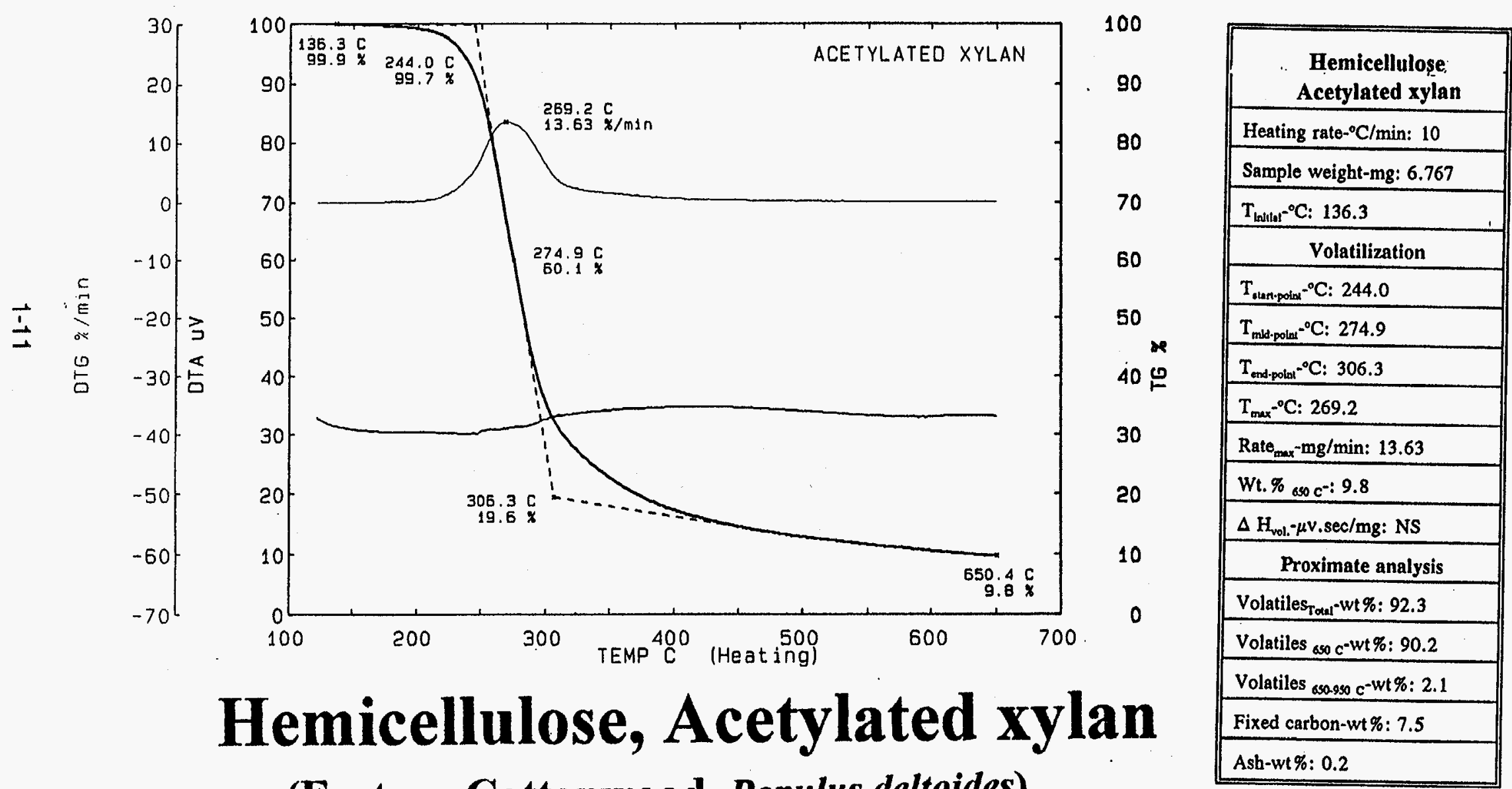

(Eastern Cottonwood, Populus deltoides) 

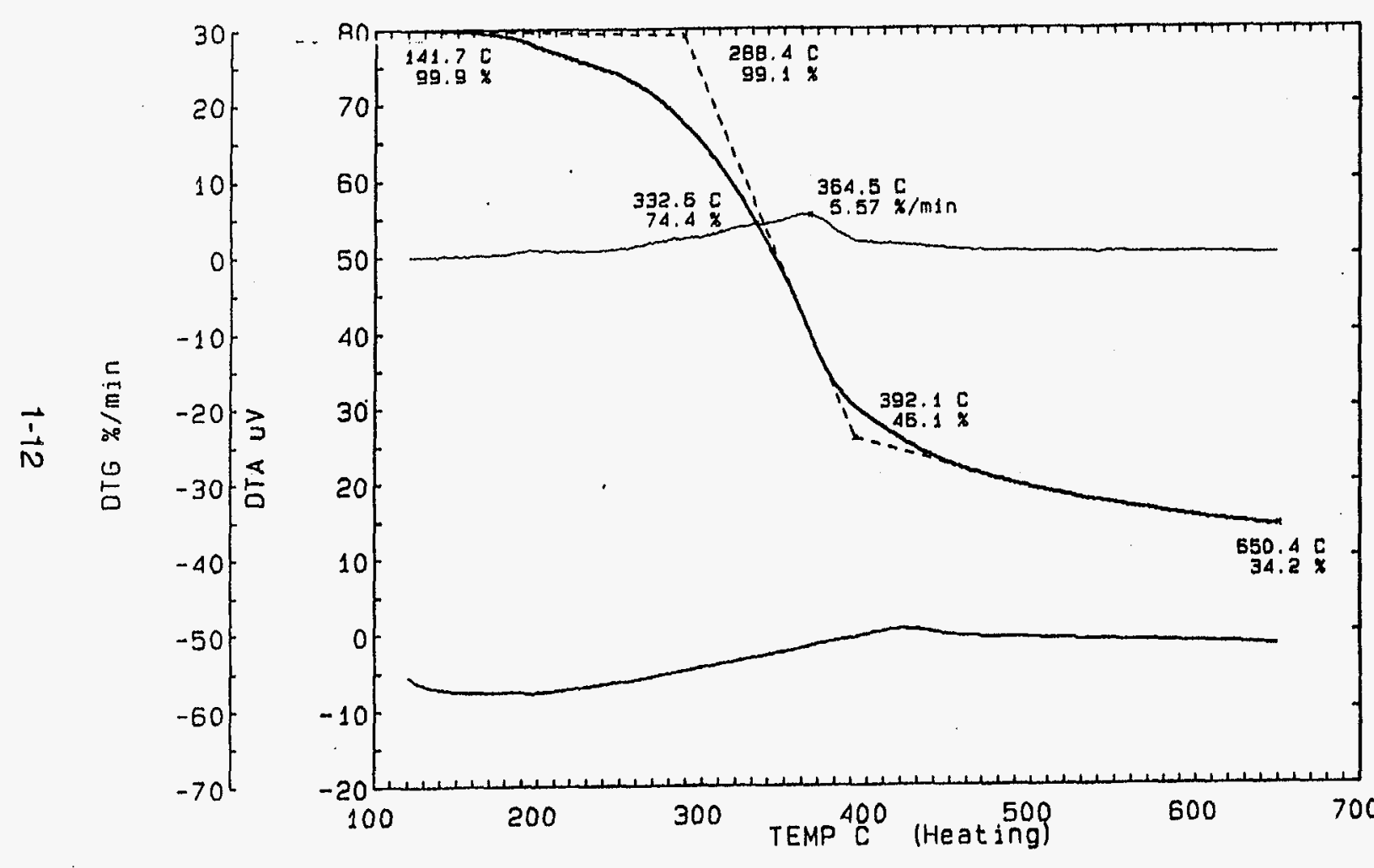

100

90

80

70

60

50

Lignin- Steam exploded Aspen (Populus tremuloides)

\begin{tabular}{|c|}
\hline $\begin{array}{c}\text { Lignin, Aspen } \\
\text { (Populus tremuloides) }\end{array}$ \\
\hline Heating rate- ${ }^{\circ} \mathrm{C} / \mathrm{min}: 10$ \\
\hline Samplo weight-mg: 8.666 \\
\hline $\mathrm{T}_{\text {baliw) }}{ }^{\circ} \mathrm{C}: 141.7$ \\
\hline Volatilization \\
\hline$T_{\text {aurmatat }} \mathrm{C}: 28 \overline{8} .4$ \\
\hline 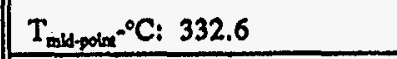 \\
\hline$T_{\text {madpotax }}{ }^{\circ} \mathrm{C}: 392.1$ \\
\hline$T_{\max }{ }^{\circ} \mathrm{C}: \cdot 364.5$ \\
\hline Rate $_{\operatorname{mex}}-\mathrm{mg} / \mathrm{min}: 5.57$ \\
\hline Wt. $\%_{\text {oss }} c^{-:} 34.2$ \\
\hline$\Delta \mathrm{H}_{\mathrm{val}}-\mu \mathrm{v} . \mathrm{sec} / \mathrm{mg}: \mathrm{NS}$ \\
\hline Proximate analysis \\
\hline Volatiles ${ }_{\text {Toul }}-w i \%: 68.5$ \\
\hline Volatiles $_{6 s 0} c^{-w t} \%: 65.8$ \\
\hline Volatiles $_{s 00,050} c^{-w t} \%: 2.7$ \\
\hline Fixed carbon-wt\%: 30.8 \\
\hline Ash-wt\%: 0.7 \\
\hline
\end{tabular}



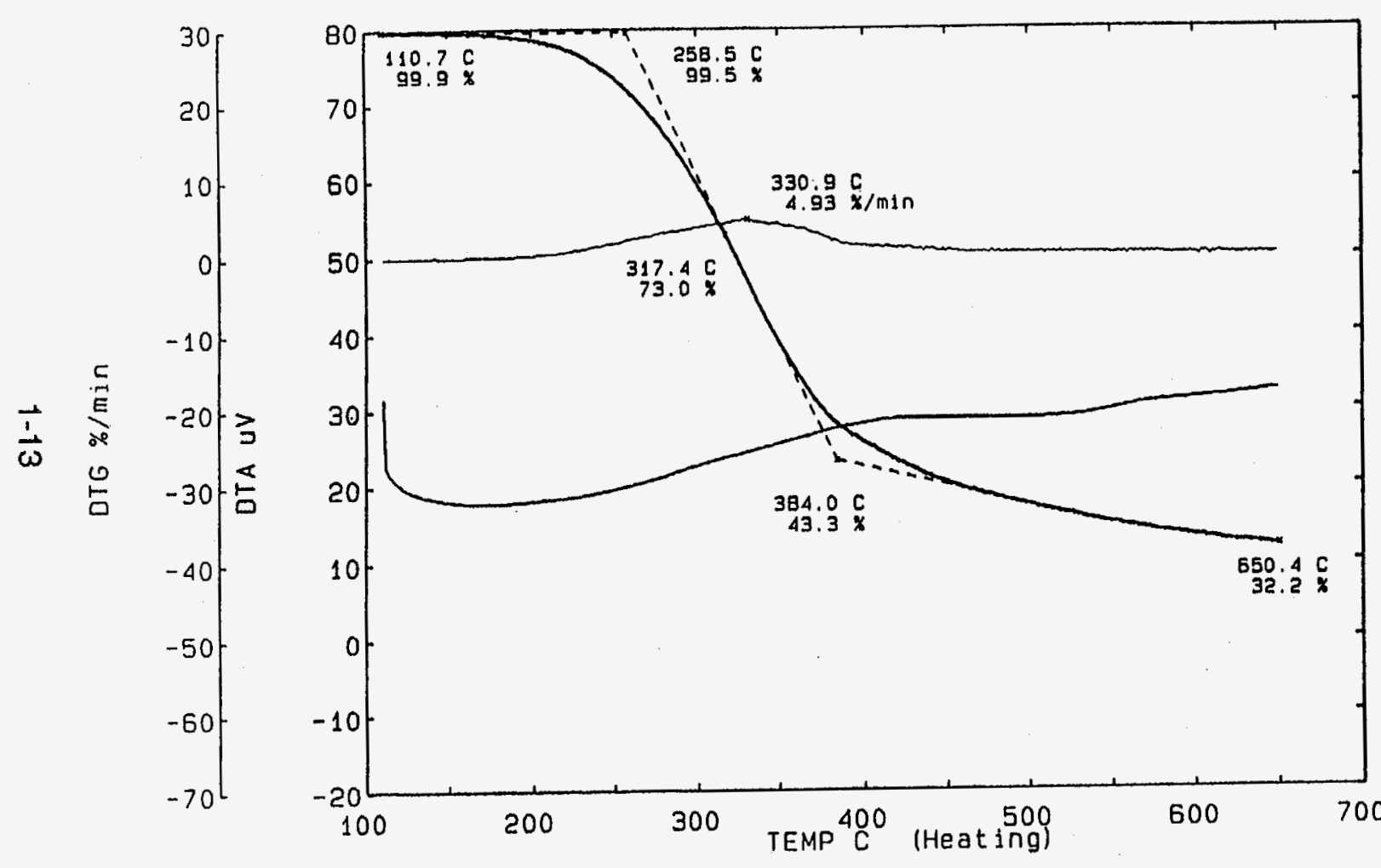

100

Lignin , Ball milled wood - (Populus deltoides) 

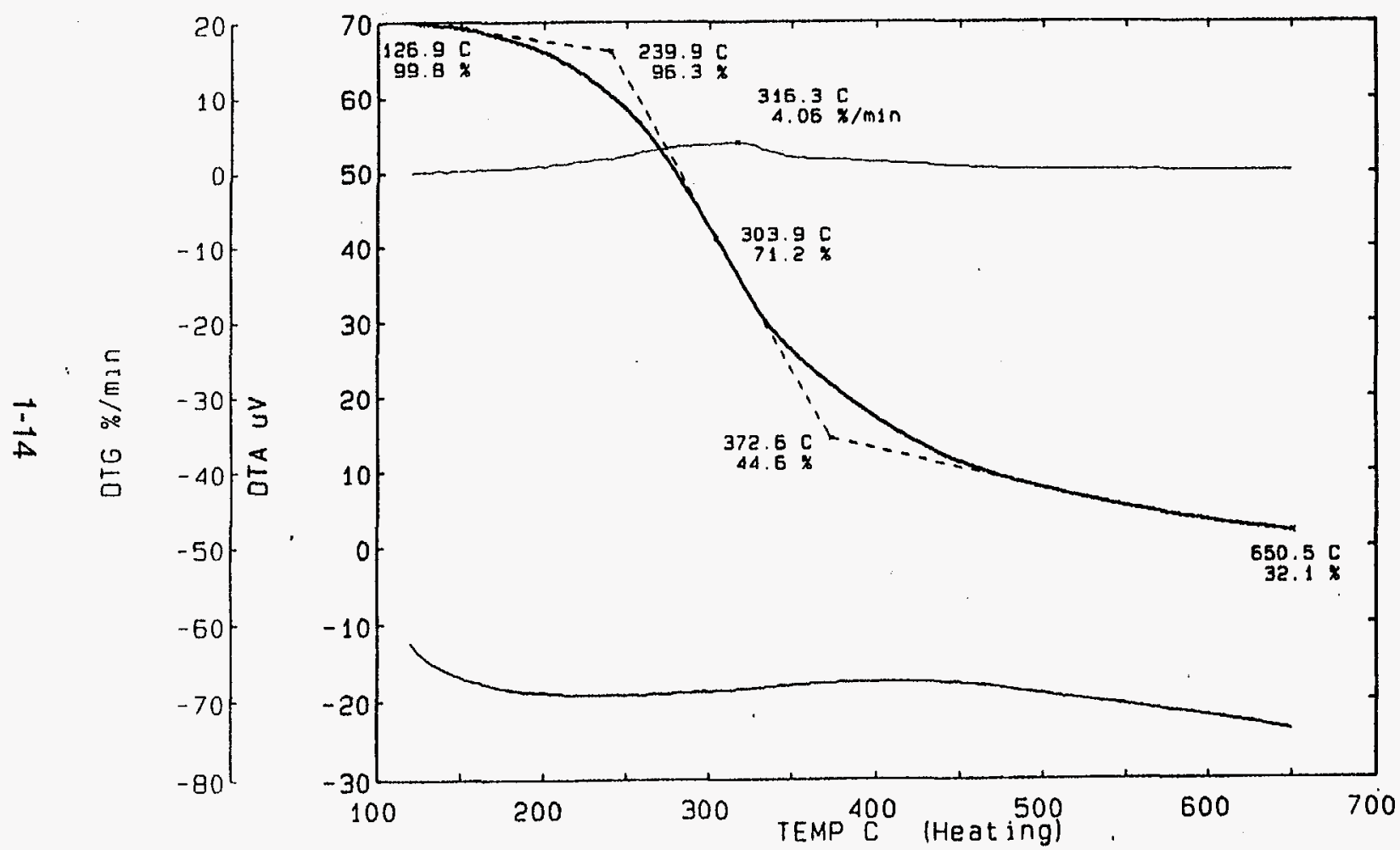

\begin{tabular}{|c|c|}
\hline 90 & $\begin{array}{l}\text { Brown Rotted Wood } \\
\text { (S. pine by P. placenta) }\end{array}$ \\
\hline \multirow{2}{*}{80} & Heating rale-- ${ }^{\circ} \mathrm{C} / \mathrm{min}: 10$ \\
\hline & Sample weight-mg: 7.657 \\
\hline 70 & $T_{\text {trinlatal }}{ }^{\circ} \mathrm{C}: 126.9$ \\
\hline \multirow{2}{*}{60} & Volatilization \\
\hline & $T_{\text {aun.potx }}{ }^{\circ} \mathrm{C}: 239.9$ \\
\hline 50 & $\mathrm{~T}_{\text {med-point }}{ }^{-} \mathrm{C}: \quad 303.9$ \\
\hline \multirow{2}{*}{$40 \cong$} & $T_{\text {end podrt }}{ }^{\circ} \mathrm{C}: 372.6$ \\
\hline & $\mathrm{T}_{\max }{ }^{\circ} \mathrm{C}: 316.3$ \\
\hline 30 & Rate $_{\mathrm{rms}}-\mathrm{mg} / \mathrm{min}: 4.06$ \\
\hline \multirow{2}{*}{20} & $W_{t} . \%_{0 s 0} c^{-}: 32.1$ \\
\hline & $\Delta \mathrm{H}_{\mathrm{vol}} \cdot \mu \mathrm{v} \cdot \mathrm{sec} / \mathrm{mg}: \mathrm{NS}$ \\
\hline 10 & Proximate analysis \\
\hline \multirow{5}{*}{0} & Volatiles $_{\text {Toul }}-w t \%: 72.8$ \\
\hline & Volatiles $_{s s o c}-w t \%: 67.9$ \\
\hline & 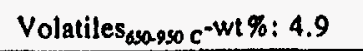 \\
\hline & Fixed carbon-wt\%: 3.8 \\
\hline & Ash-w1\%: 23.4 \\
\hline
\end{tabular}

(S. pinus by P. placenta) 


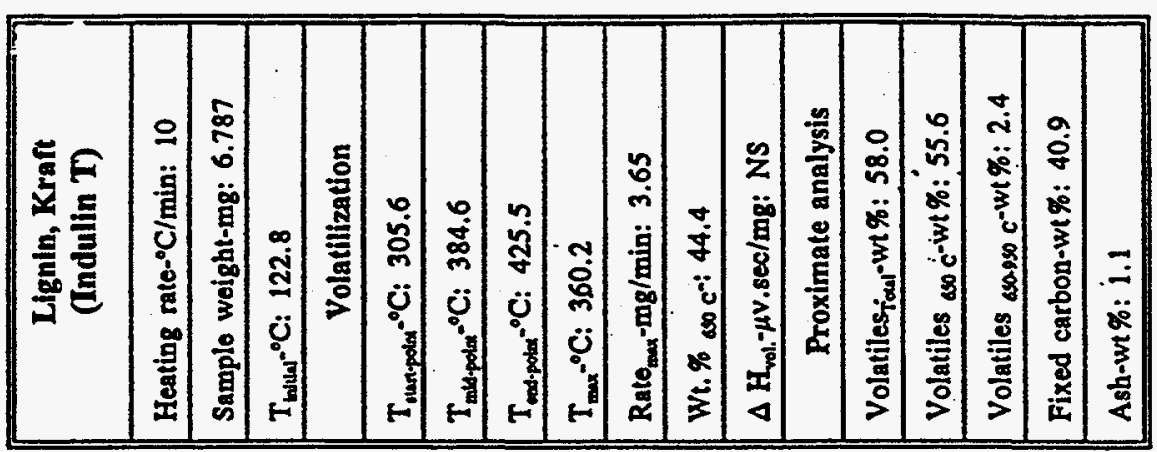

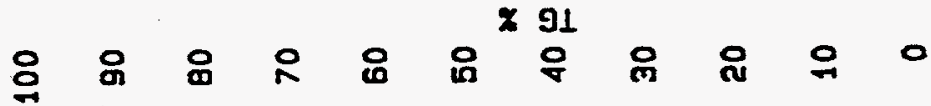

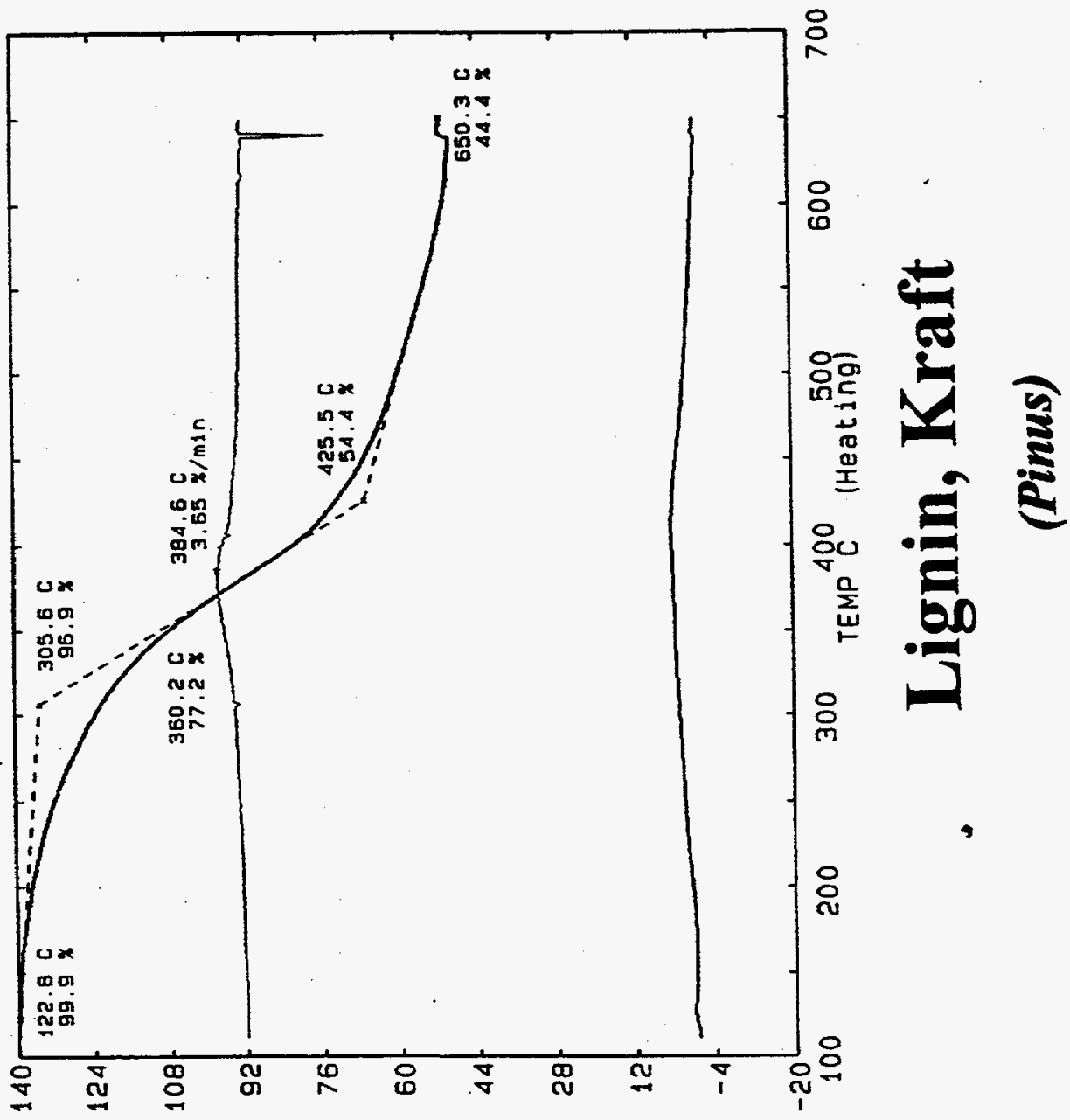

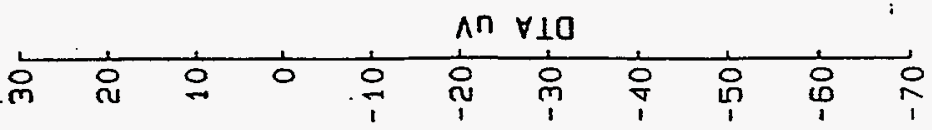

Uโพแ/\% 910 

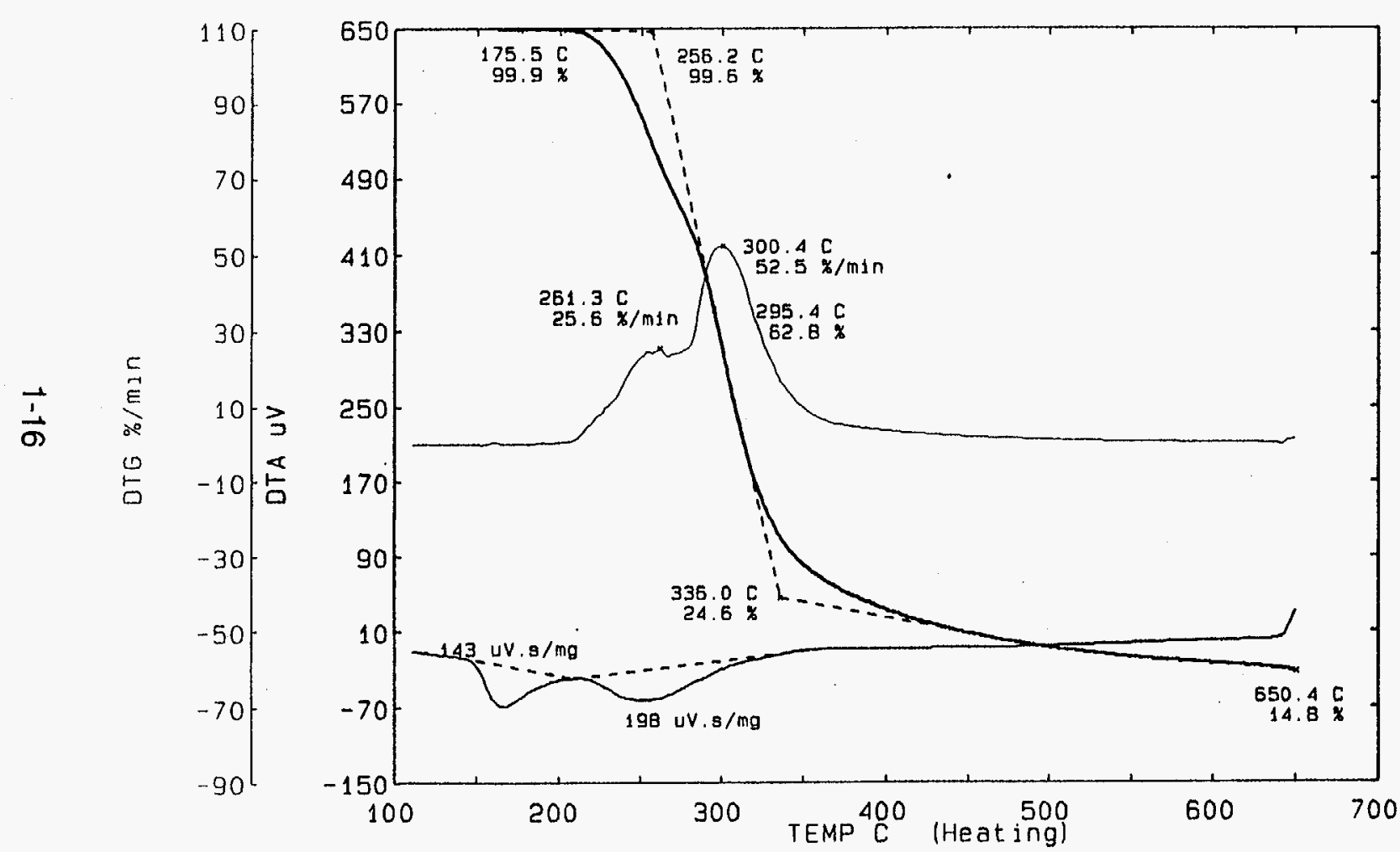

100

90

BO

70

EO

50

se

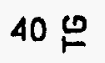

30

20

10

Glucose

(Baker)

\begin{tabular}{|c|}
\hline $\begin{array}{l}\text { Glucose } \\
\text { (Baker) }\end{array}$ \\
\hline
\end{tabular}

Heating rate- $-{ }^{\circ} \mathrm{C} / \mathrm{min}: 10$

Sample weight-mg: 13.09

$\mathrm{T}_{\text {inlitat }}{ }^{\circ} \mathrm{C}: 175.5$

\begin{tabular}{|l} 
Volatilizal \\
\hline$T_{\text {sunn-polum }}{ }^{\circ} \mathrm{C}: 256.2$
\end{tabular}

$T_{\text {mid-poinx }}{ }^{\circ} \mathrm{C}: 295.4$

$\mathrm{T}_{\text {end.polm }}{ }^{-0} \mathrm{C}: \mathbf{3 3 6 . 0}$

$T_{\max }{ }^{\circ} \mathrm{C}: 300.4$

Rate $_{\max }-\mathrm{mg} / \mathrm{min}: 52.5$

Wt. \% 650 c $=14.8$

$\Delta \mathrm{H}_{\mathrm{vol}}, \mu \mathrm{v} . \mathrm{sec} / \mathrm{mg}: 143,198$

Proximate analysis

Volatiles $_{\text {Toul }}-\mathrm{wt} \%: 85.7$

Volatiles ${ }_{050} \mathrm{c}^{-\mathrm{w}} \%: 85.2$

Volatiles $9.0 \mathrm{c} \cdot \mathrm{w} 1 \%: 0.5$

Fixed carbon-wt\%: 14.1

Ash-wt\%: 0.2 

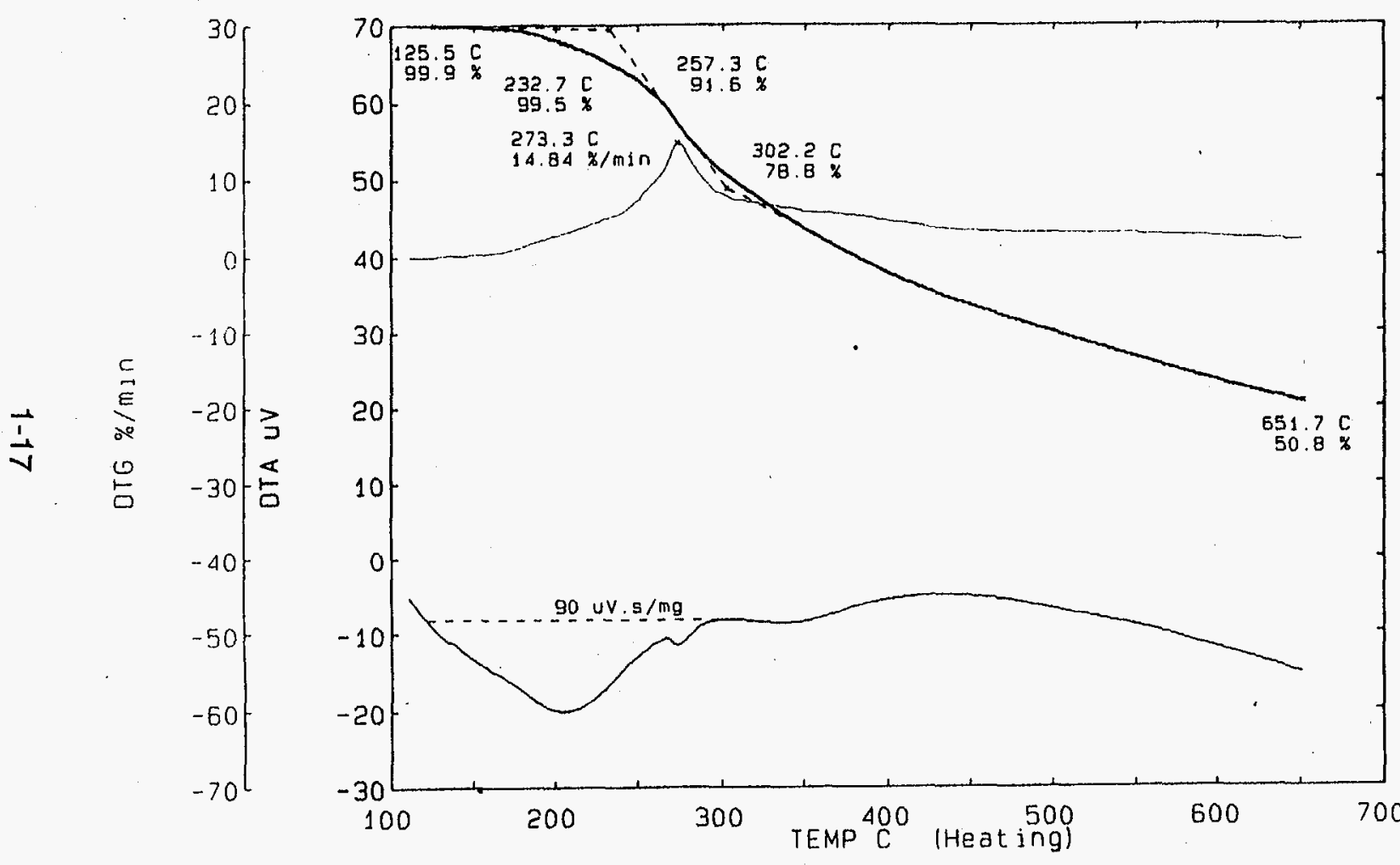

Wattle tanin

(Acacia sp.)

\begin{tabular}{|c|c|}
\hline \\
\hline 90 & $\begin{array}{c}\text { Tannin, wattle } \\
\text { (Acacia sp.) }\end{array}$ \\
\hline \multirow{2}{*}{80} & Heating rate ${ }^{\circ} \mathrm{C} / \mathrm{min}: 10$ \\
\hline & Sample weight-mg: 18.09 \\
\hline 70 & $T_{\text {invitis }}{ }^{\circ} \mathrm{C}: 125.5$ \\
\hline \multirow{2}{*}{60} & Volatilization \\
\hline & $T_{\text {stern-poinn }}{ }^{\circ} \mathrm{C}: 232.7$ \\
\hline 50 & $\mathrm{~T}_{\text {mid.poin }}{ }^{-} \mathrm{C}: 257.3$ \\
\hline \multirow{2}{*}{$40 \stackrel{0}{\circ}$} & $T_{\text {end.point }}{ }^{\circ} \mathrm{C}: 302.2$ \\
\hline & $T_{\text {max }}{ }^{\circ} \mathrm{C}: 273.3$ \\
\hline 30 & Rate $_{\operatorname{man}}-\mathrm{mg} / \mathrm{min}: 14.8$ \\
\hline \multirow{2}{*}{20} & $W_{t .} \%_{S S O C}: 51$ \\
\hline & $\Delta H_{\text {vol }}-\mu v \cdot \mathrm{sec} / \mathrm{mg}: 90$ \\
\hline 10 & Proximate analysis \\
\hline \multirow{5}{*}{0} & Volatiles $_{\text {rotal }}-w_{1} \%: 55.2$ \\
\hline & Volatiles $_{6,00} c^{-w 1 \%: 49}$ \\
\hline & Volatiles $_{650-950} c^{-w t} \%: 6.2$ \\
\hline & Fixed carbon-wt\%: 44 \\
\hline & Ash-wt \%: 0.8 \\
\hline
\end{tabular}




\section{Chapter 2}

\section{NATURAL BIOMASS}

The energy potentially available from biomass has been estimated to be about $20 \%$ of US. energy consumption [OTA, 1980]. The distribution of these sources amongst the various biomass forms are shown in Table 2.1.

Table 2.1 - Biomass resources as energy in the US. [Inman, 1981]

\begin{tabular}{lrc}
\multicolumn{1}{c}{ RESOURCE } & $\mathbf{1 0}^{6}$ Dry Tons $/$ Year & Quads/yr $^{\mathrm{a}}$ \\
Crop Residues & 278.0 & 4.15 \\
Animal manures & 26.5 & 0.33 \\
Unused mill residues & 24.1 & 0.41 \\
Logging residues & 83.2 & 1.41 \\
Municipal solid wastes & 130.0 & 1.63 \\
Standing forests & 384.0 & 6.51 \\
\multicolumn{1}{c}{ Totals } & 925.8 & 14.44 \\
& & $\mathrm{a}_{1 \text { quad }}=10^{15_{\text {Btury. }}}$
\end{tabular}

To date, most of the biomass for energy has come from existing residues. However, a great deal of experimental work has been done on energy farming, for raising high yield biomass crops such as fast growing trees and grasses directly for energy [Inman, 1981].

\subsection{Softwoods}

Softwood is the common name for tree species more properly called coniferous. (The name is a poor choice because many hardwoods are often softer than some softwoods.)

Softwoods typically have larger cells and longer cellulose fibers than hardwoods, and so they are used primarily for paper making.

Wood energy is most easily derived from residues already collected at the mill. Mill wood and bark residues amount to 86.1 million tons/yr. of which 62 million are already used for energy. In addition, there is about 83 million tons/yr. of residues left in the forest during logging operations and some of this could be harvested for energy [inman, 1981]. 
By far the largest existing resource is the surplus and non commercial components of the standing forests, about 383 million tons/yr. [Inman, 1981]. The harvest of this resource for energy would probably be closely associated with commercial timber stand improvement practices and harvest. However, environmental concerns would have to be addressed before any harvesting is done, but foresters agree that a managed forest is in many cases healthier for both flora and fauna.

\subsection{Hardwoods}

Hardwood is the common name for tree species more properly called deciduous, or leaf bearing trees. Many hardwoods are relatively dense and often ornamental, so they are used in applications where this is important. Many of the fastest growing, high biomass productivity trees, such as poplars, are called hardwood but are actually quite soft, so this term is not very accurate. The species growing in the tropics are often quite different from those in temperate climates and will be of especial interest for energy in developing countries.

\subsection{Agricultural Residues}

Agricultural residues are the straws, the hulls, the seeds, the linters and other by-products of agriculture. They are widely available at low or negative (on site) cost for energy use.

While woods have low ash contents of $0.1 \%$ to $2 \%$, agricultural residues typically have 2$6 \%$ and in some cases up to $20 \%$ (rice hulls). Annual availability of crop residues is shown in Table 2.2

Table 2.2 - Annual Availability of Crop Residues [Inman, 1981]

RESIDUE CATEGORY

Com and sorghum (field)

Small grains and grasses (field)

Other crops

Collected residues

Total

\section{MILLION DRY TONSMR.}

\author{
96.6
}

131.8

42.3

278.0

\subsection{Aquatic Biomass}

Aquatic biomass is a potential source for energy. On the plus side, it grows in the oceans which cover $80 \%$ of the globe, in lakes, streams and rivers while, on the other side, aquatic biomass typically contains a great deal of water and can be high in ash. There have been many proposals to collect and use various forms of aquatic biomass for energy, but none have become commercial yet. Indications are that if these resources are properly tapped then algae can turn out to be a major oil (triglyceride) producing source. 


\subsection{Observations}

Since Table 1.1 shows that there is little difference between the $\mathrm{C} / \mathrm{H} / \mathrm{L}$ (cellulose, hemicellulose and lignin) composition of hardwood and softwood, it is not surprising that their thermograms are quite similar, and appear to be the arithmetic sums of the behavior of $\mathrm{C}, \mathrm{H}$ and $\mathrm{L}$. (This is also shown in Chapter 8.) This is also true of many of the agricultural specimens. One may even expect that the thermograms could be deconvoluted to give the proportions of $\mathrm{C} / \mathrm{H} / \mathrm{L}$. (However, ash plays a major catalytic role in determining volatility, so would have to be removed before this could be attempted.)

It is clearly seed that as a class agricultural materials contain more ash and produce more fixed carbon than woody materials. This suggests that agricultural residues could become a major source of charcoal, with briquetting for fuel use in developing countries. This would decrease the deforestation in these countries, since the residues are of little current value

The aquatic specimens are unique in having a sudden loss of mass at about $120^{\circ} \mathrm{C}$. Since all the specimens are dried equally at $110^{\circ} \mathrm{C}$ before running the thermograms, this is puzzling. Perhaps there are small, impermeable cells containing water that explode at this temperature. 


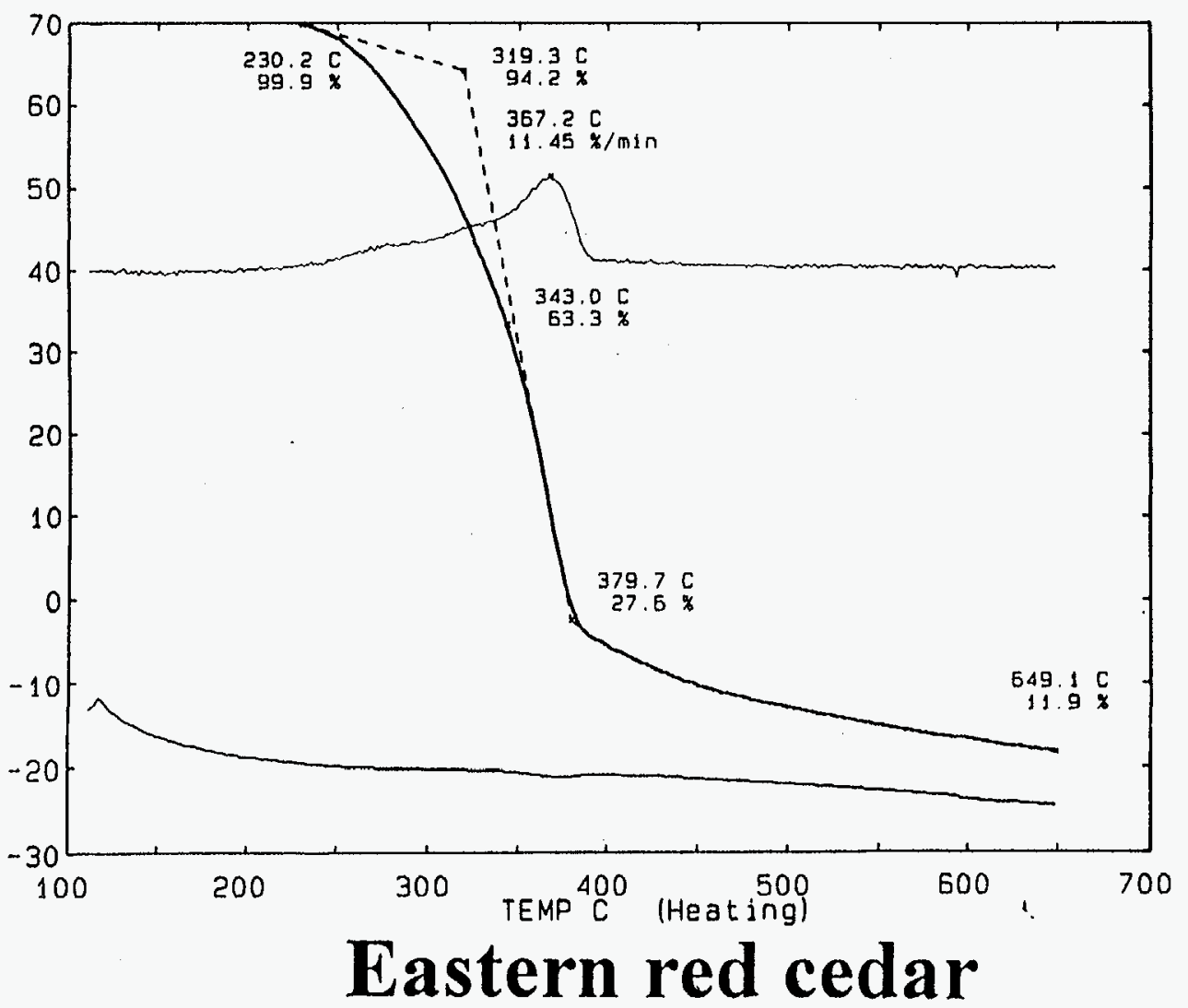

100

90

80

70

60

50

$40 \cong$

30

20

10

0

\section{(Juniperus Virginiana)}

\begin{tabular}{|c|}
\hline $\begin{array}{l}\text { Eastern red cedar } \\
\text { (Juniperus virginiana) }\end{array}$ \\
\hline Heating rate- $-^{\circ} \mathrm{C} / \mathrm{min}: 10$ \\
\hline Sample weight-mg: 11.09 \\
\hline $\mathrm{T}_{\text {inimat }}{ }^{-0} \mathrm{C}: 230.2$ \\
\hline Volatilization \\
\hline $\mathrm{T}_{\text {aur.popdx }}{ }^{-0} \mathrm{C}: 319.3$ \\
\hline $\mathrm{T}_{\text {mblpotax }}{ }^{\circ} \mathrm{C}: 343.0$ \\
\hline $\mathrm{T}_{\text {ent.polat }}{ }^{\circ} \mathrm{C}: 379.7$ \\
\hline$T_{\max } 0^{\circ} \mathrm{C}: 367.2$ \\
\hline Rate $_{\text {max }}-\mathrm{mg} / \mathrm{min}: 11.45$ \\
\hline Wt. \% $650 c^{-:}: 11.9$ \\
\hline$\Delta H_{\text {vol }}-\mu \mathrm{v} . \sec / \mathrm{mg}: \mathrm{NS}$ \\
\hline Proximate analysis \\
\hline Volatiles $_{\text {Tout }_{1}}-w t \%: 93.5$ \\
\hline Volatiles ${ }_{6 s 0} c^{-w t} \%: 88.1$ \\
\hline Volatiles ${ }_{\text {ssa. }}$ so $c^{-w 1} \%: 5.4$ \\
\hline Fixed carbon-wt $\%: 5.0$ \\
\hline Ash-wt \%: 1.5 \\
\hline
\end{tabular}



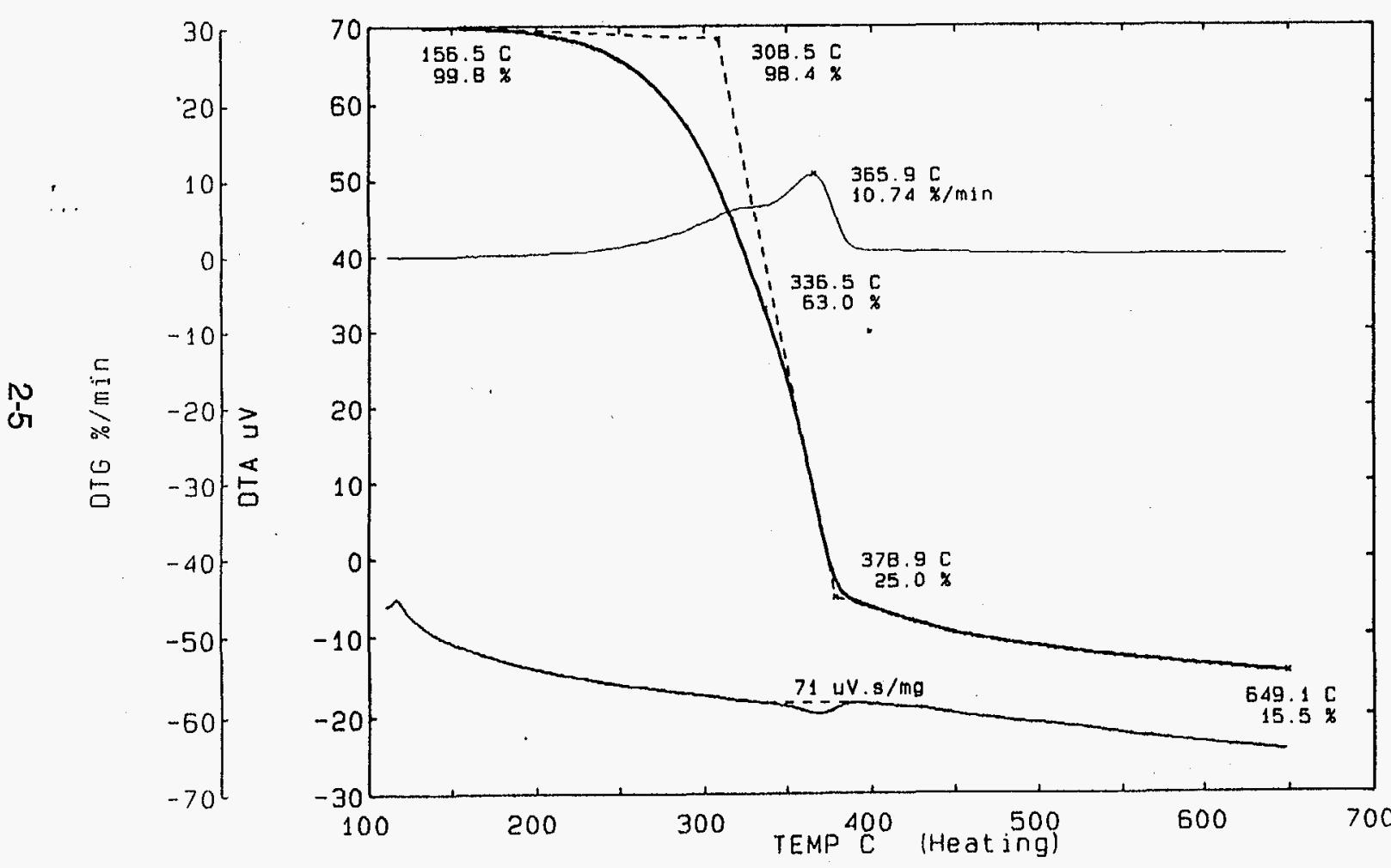

100

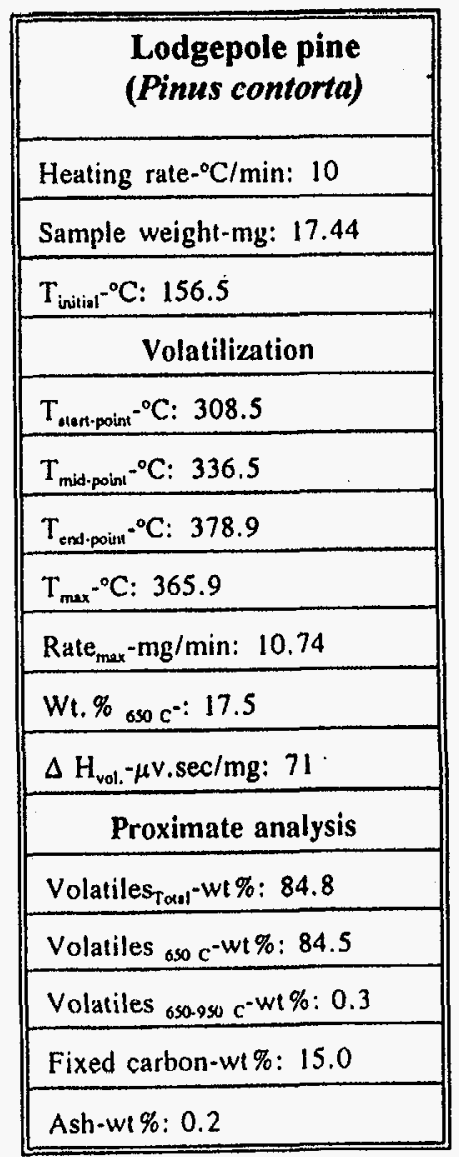




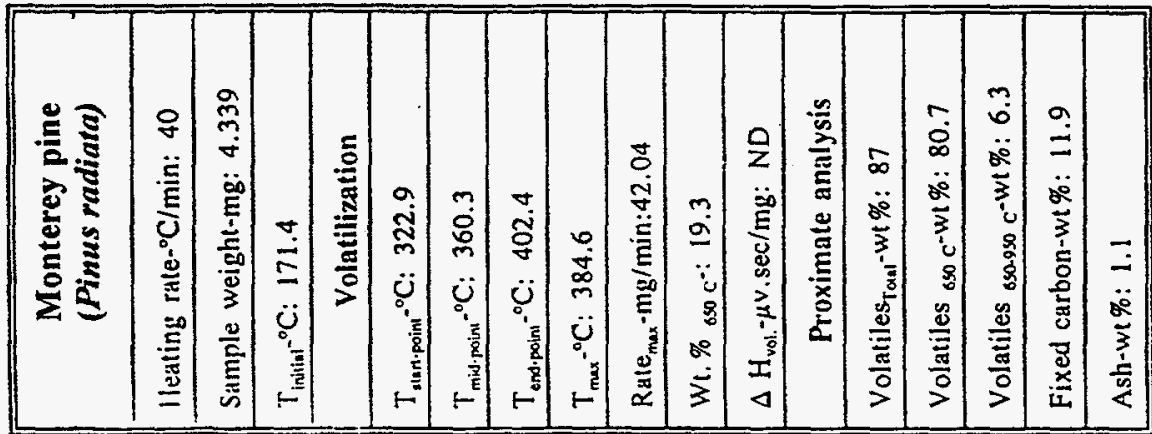

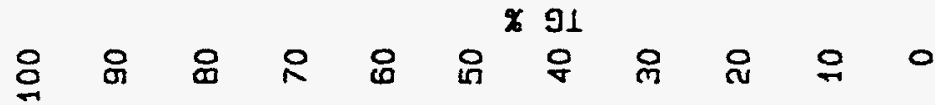
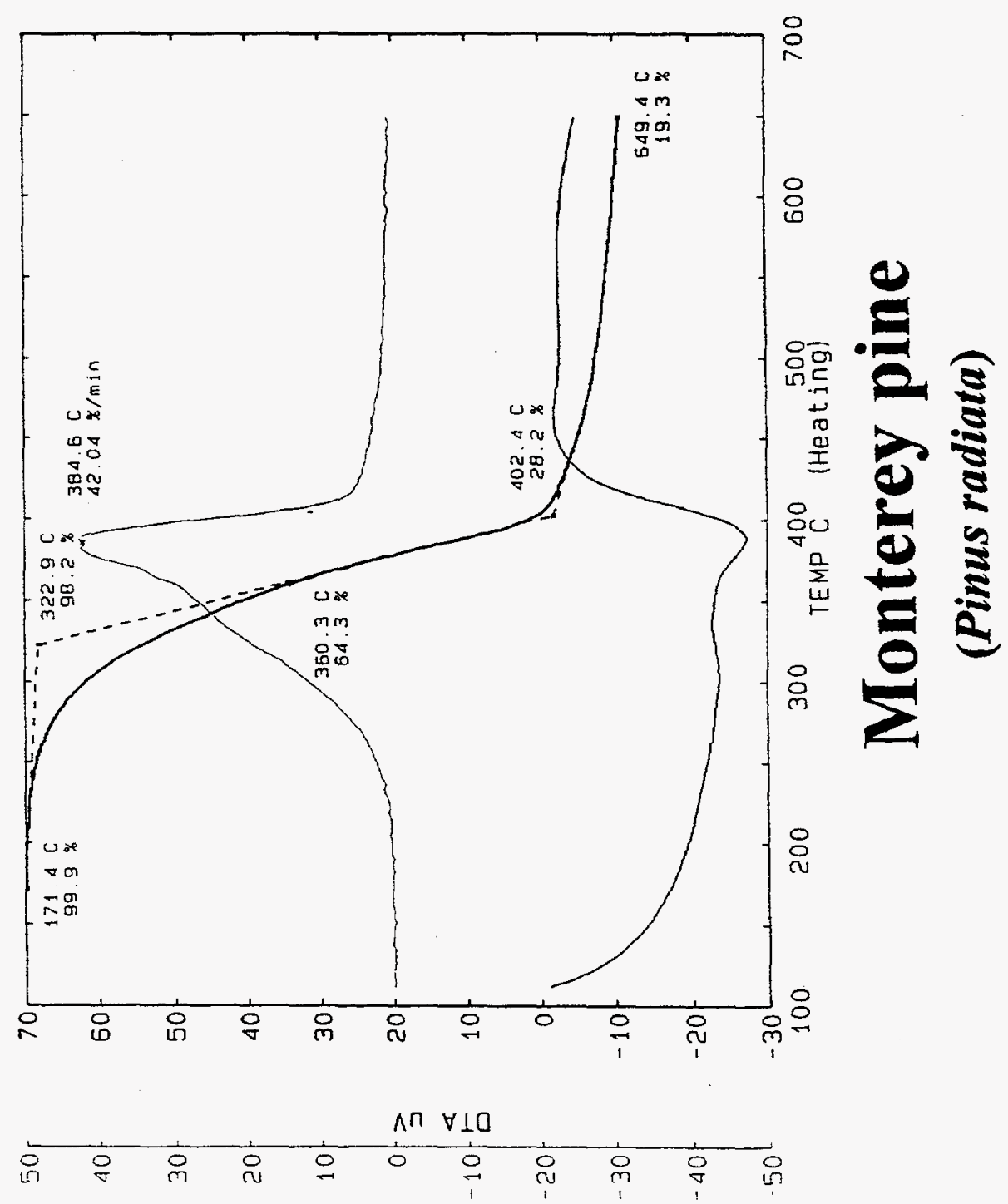

$u[w / \% 9 ! 0$ 

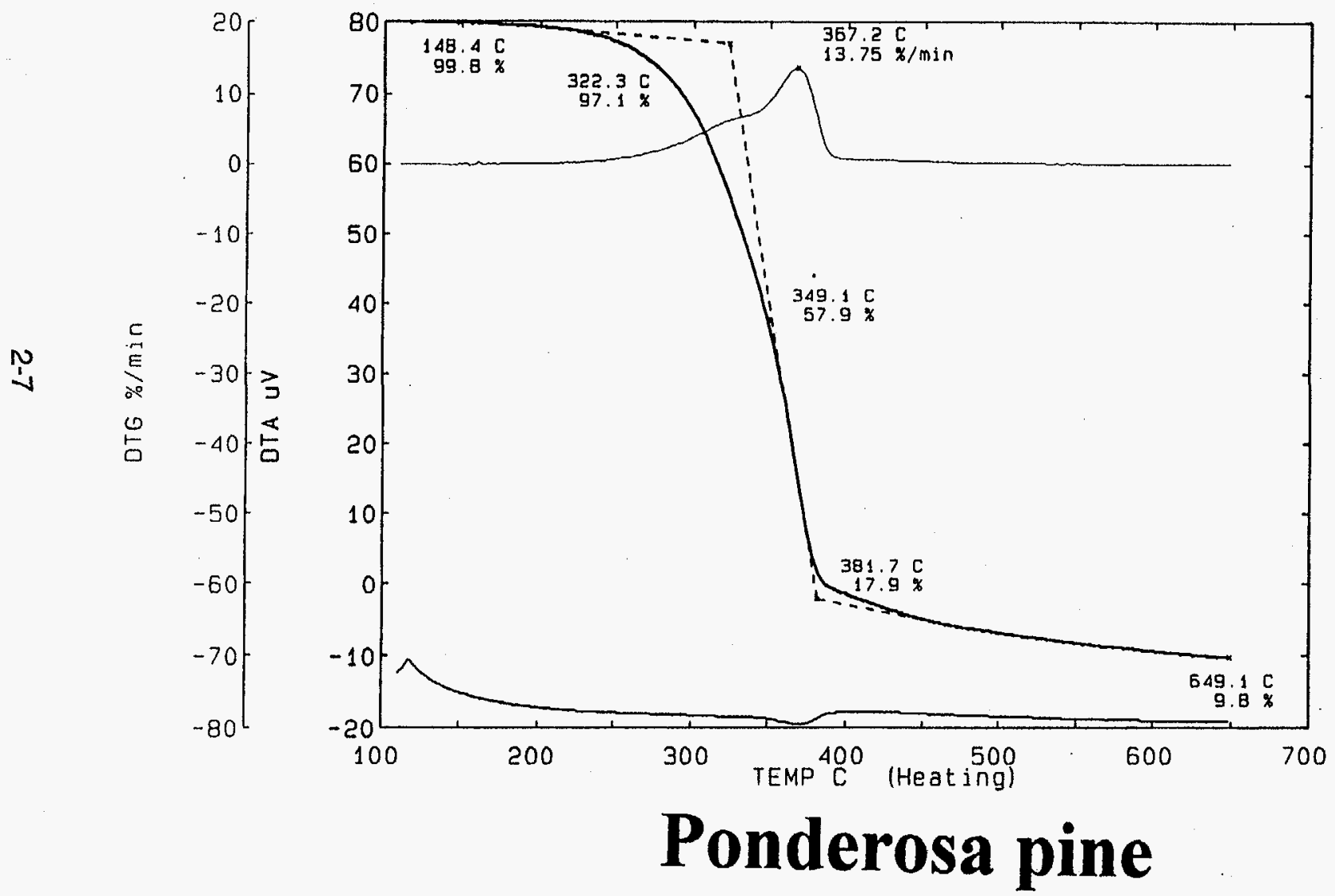

100

90

80

70

60

50

x

$40 \stackrel{0}{2}$

30

20

10

0

(Pinus ponderosa)

\begin{tabular}{|c|}
\hline $\begin{array}{l}\text { Ponderosa pine } \\
\text { (Pinus ponderosa) }\end{array}$ \\
\hline Heating rate- ${ }^{\circ} \mathrm{C} / \mathrm{min}: 10$ \\
\hline Sample weight-mg: 3.185 \\
\hline $\mathrm{T}_{\text {ininiol }}{ }^{-{ }^{\circ} \mathrm{C}:} \quad 148.4$ \\
\hline Volatilization \\
\hline $\mathrm{T}_{\text {tion-poin: }} \mathrm{-}^{\circ} \mathrm{C}: 322.3$ \\
\hline $\mathrm{T}_{\text {mid-poim }}{ }^{\circ} \mathrm{C}: 349.1$ \\
\hline$T_{\text {end-poini }}{ }^{\circ} \mathrm{C}: 381.7$ \\
\hline$T_{\max }-{ }^{\circ} \mathrm{C}: 367.2$ \\
\hline Rate $_{\max }-\mathrm{mg} / \min : 13.75$ \\
\hline$W_{t} \%_{6 s 0} c^{-}: 9.8$ \\
\hline 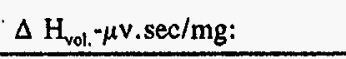 \\
\hline Proximate analysis \\
\hline Volatiles $_{\text {Tou1 }}-w 1 \%: 91.3$ \\
\hline Volatiles $_{6 s 0} c^{-w t} \%: 90.2$ \\
\hline Volatiles $_{\text {san-sso }} c^{-w t \%: 1.1}$ \\
\hline Fixed carbon-wt\%: 7.7 \\
\hline Ash-wt\%: 1.0 \\
\hline
\end{tabular}



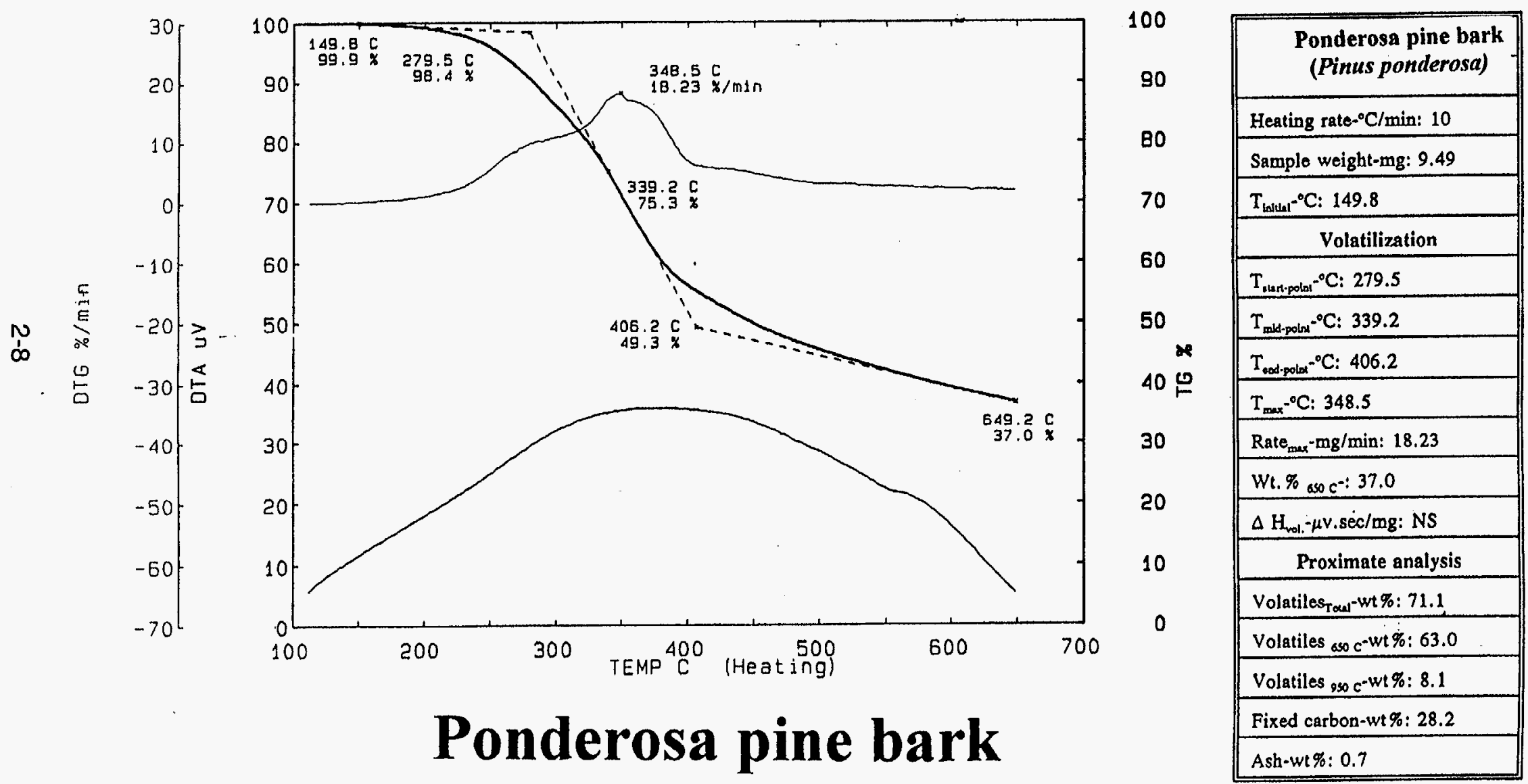

Ponderosa pine bark

(Pinus ponderosa) 

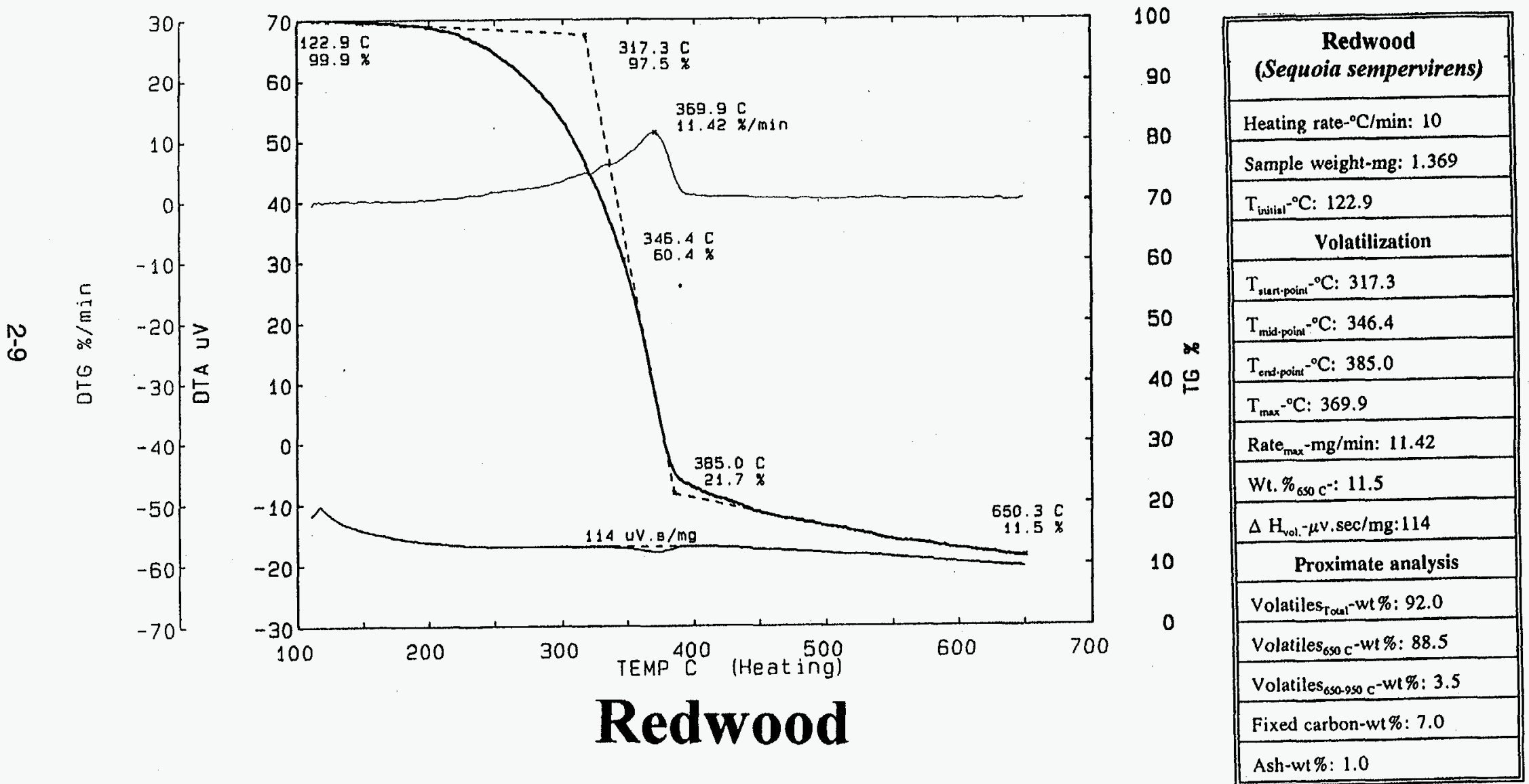

(Sequoia sempervirens) 

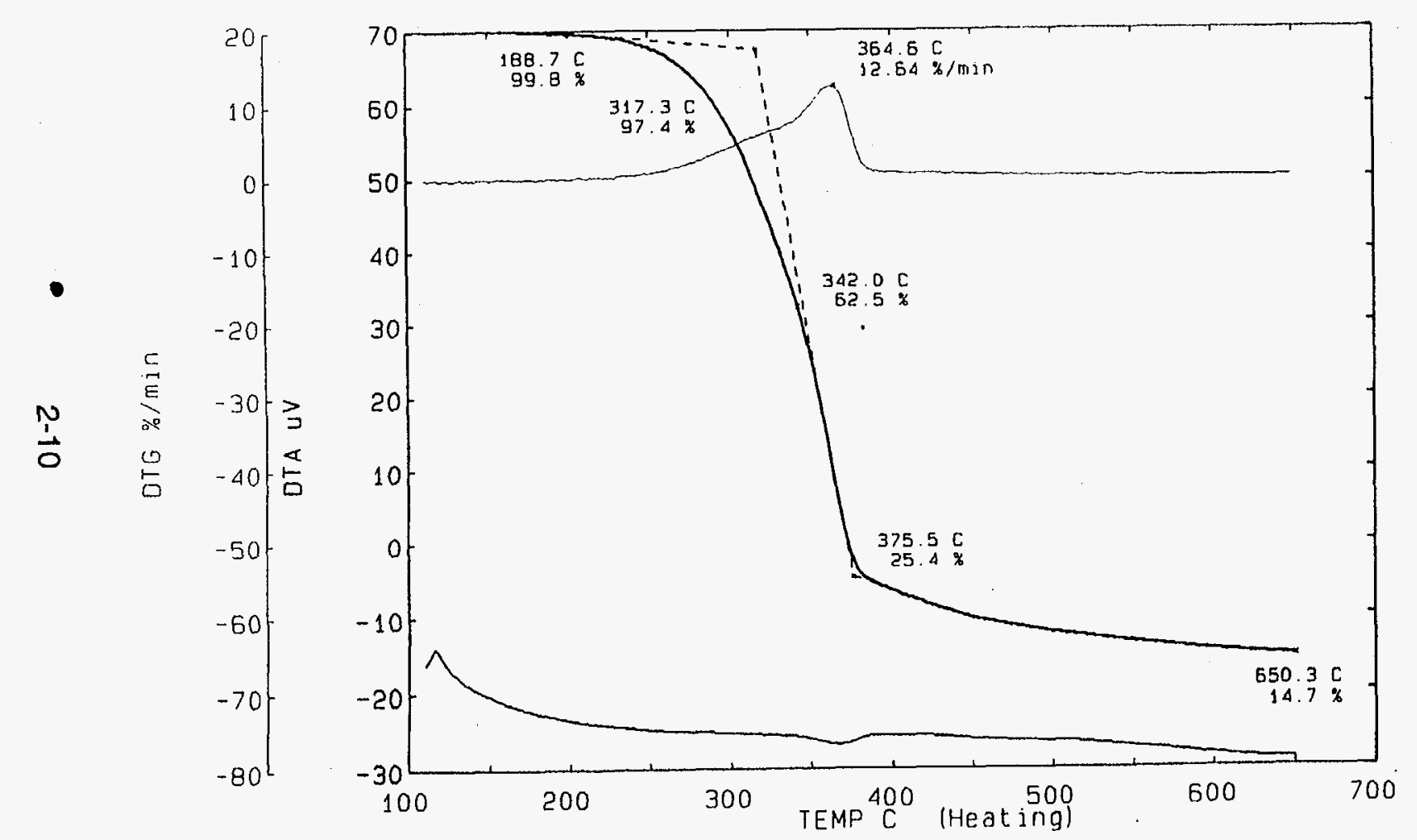

\begin{tabular}{|c|c|}
\hline 100 & $\begin{array}{c}\text { Slash pine } \\
\text { (Pinus elliottii) }\end{array}$ \\
\hline \multirow{2}{*}{80} & Heating rate- ${ }^{\circ} \mathrm{C} / \mathrm{min}: 10$ \\
\hline & Sample weight-mg: 2.978 \\
\hline 70 & $\mathrm{~T}_{\text {inititif }}{ }^{\circ} \mathrm{C}: 188.7$ \\
\hline \multirow{2}{*}{60} & Volatilization \\
\hline & $\mathrm{T}_{\text {surn-point }}{ }^{\circ} \mathrm{C}: 317.3$ \\
\hline \multirow{3}{*}{$\begin{array}{l}50 \\
40 \stackrel{2}{2}\end{array}$} & $T_{\text {mid-poin }}{ }^{-{ }^{\circ} \mathrm{C}: 342.0}$ \\
\hline & $\mathrm{T}_{\text {end popin }}{ }^{\circ} \mathrm{C}: 375.5$ \\
\hline & $T_{\text {mux }}{ }^{\circ} \mathrm{C}: 364.6$ \\
\hline 30 & Rale $_{\operatorname{mex}}-\operatorname{mg} / \min : 12.64$ \\
\hline \multirow{2}{*}{20} & WI. $\%_{650} c^{-}: 14.7$ \\
\hline & $\Delta \mathrm{H}_{\mathrm{vol}}-\mu \mathrm{v} \cdot \mathrm{sec} / \mathrm{mg}: \mathrm{NS}$ \\
\hline 10 & Proximate analysis \\
\hline \multirow{5}{*}{0} & Volatiles $_{\text {Totut }}-\mathrm{wt} \%: 88.0$ \\
\hline & 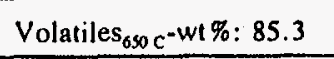 \\
\hline & Volatiles ${ }_{050.950} c^{-w t} \%: 2.7$ \\
\hline & Fixed carbon-wt $\%: 10.7$ \\
\hline & Ash-wt\%: 1.3 \\
\hline
\end{tabular}

(Pinus elliottii) 


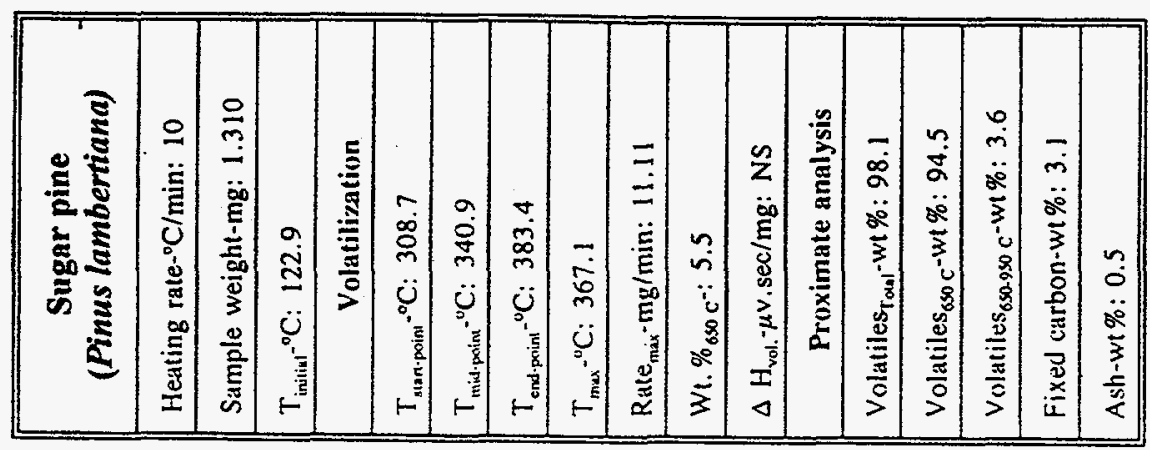

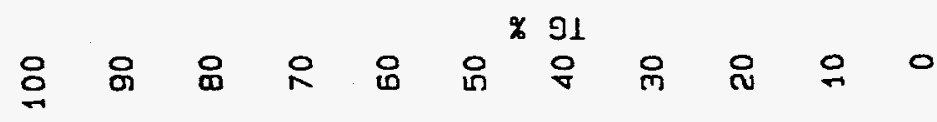
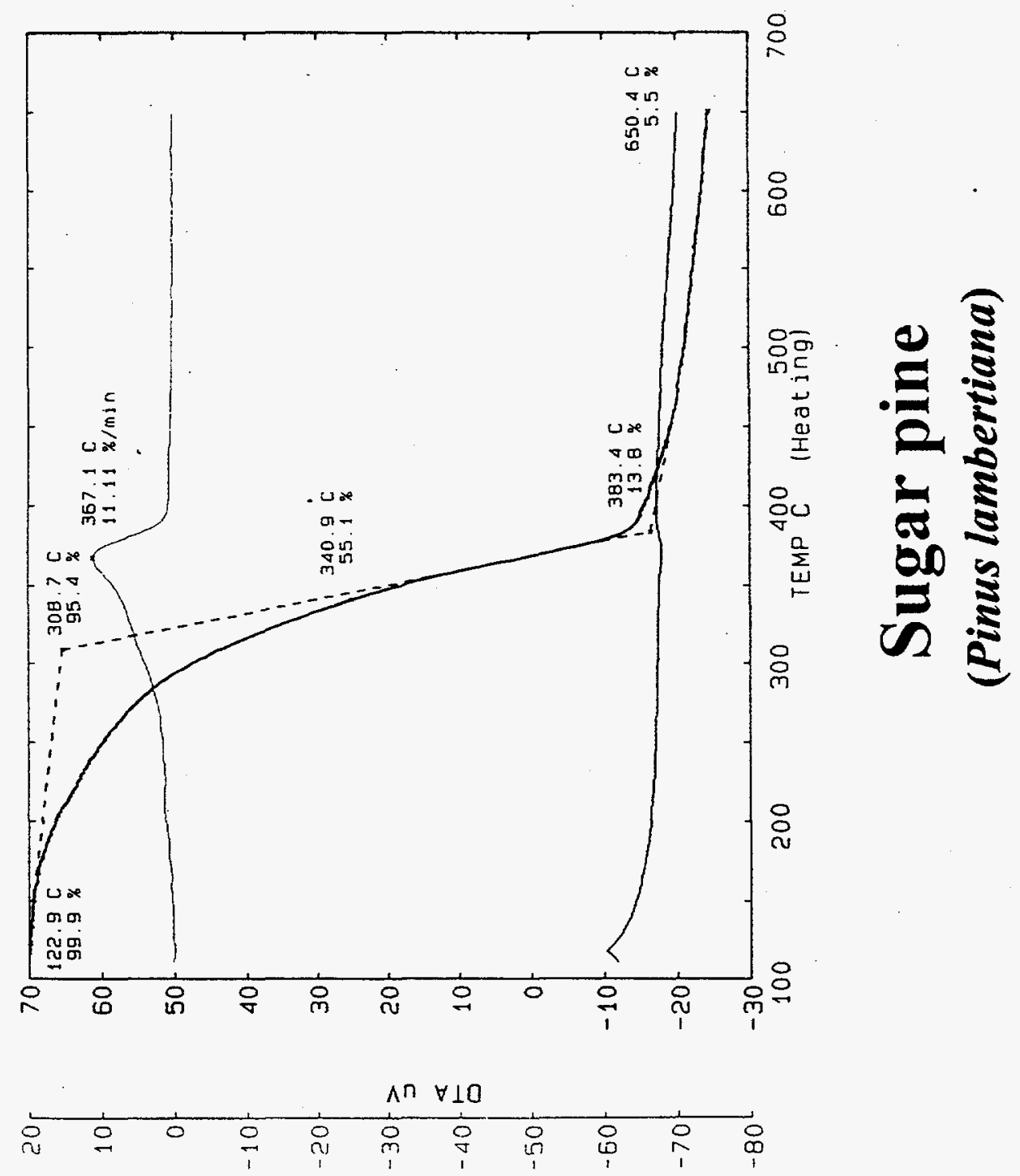

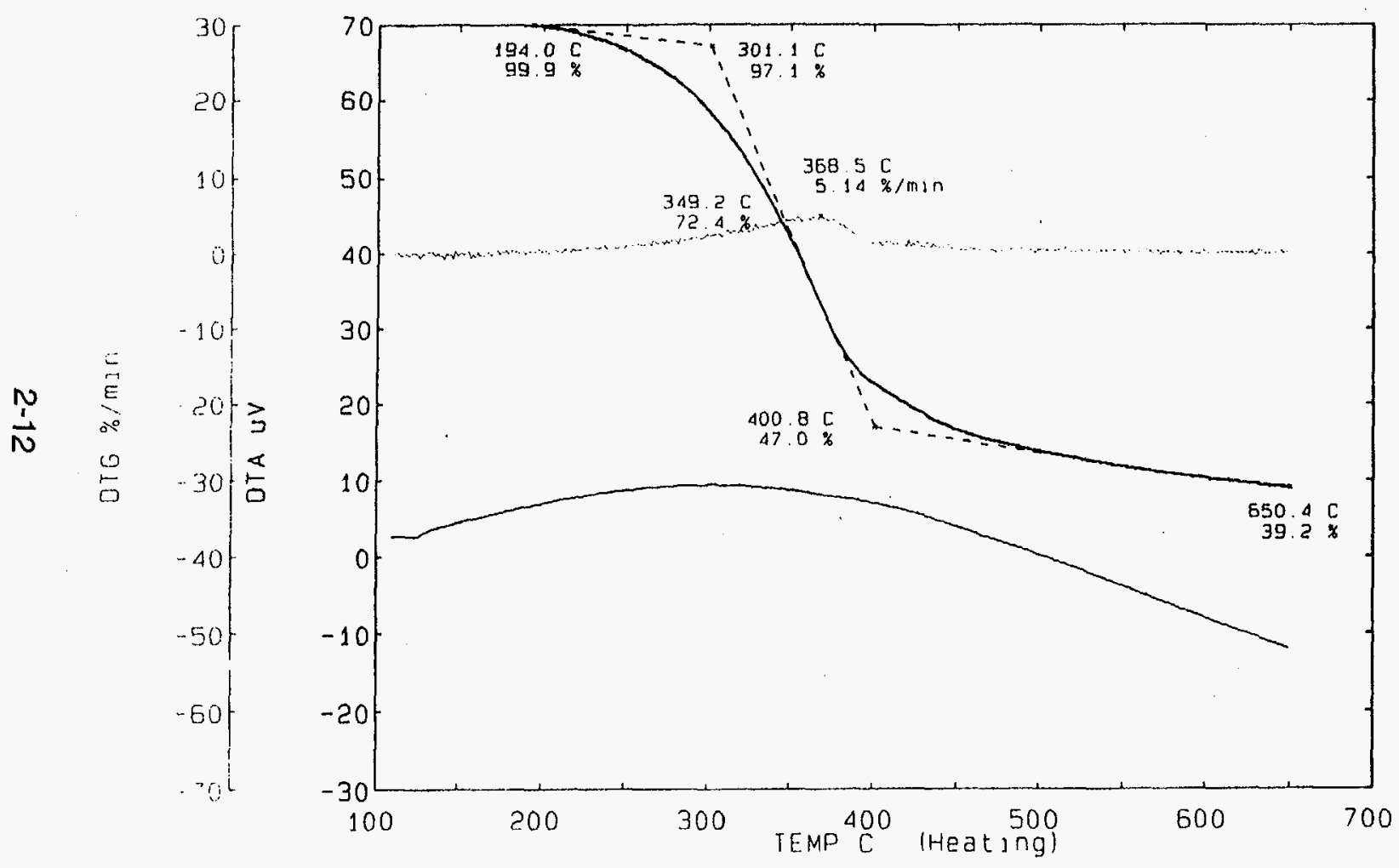

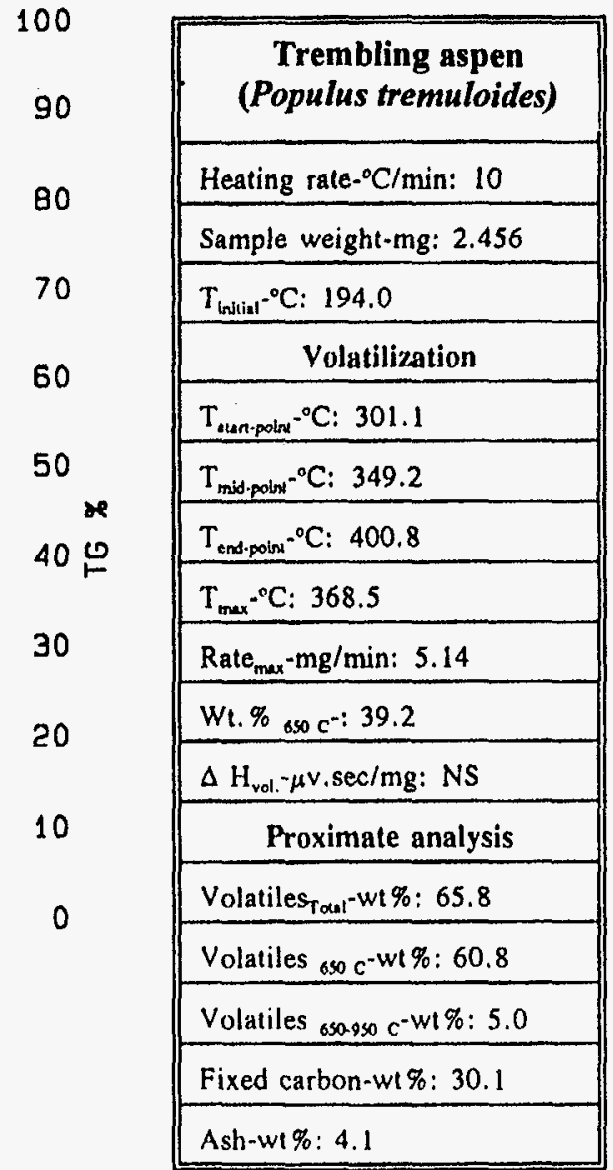



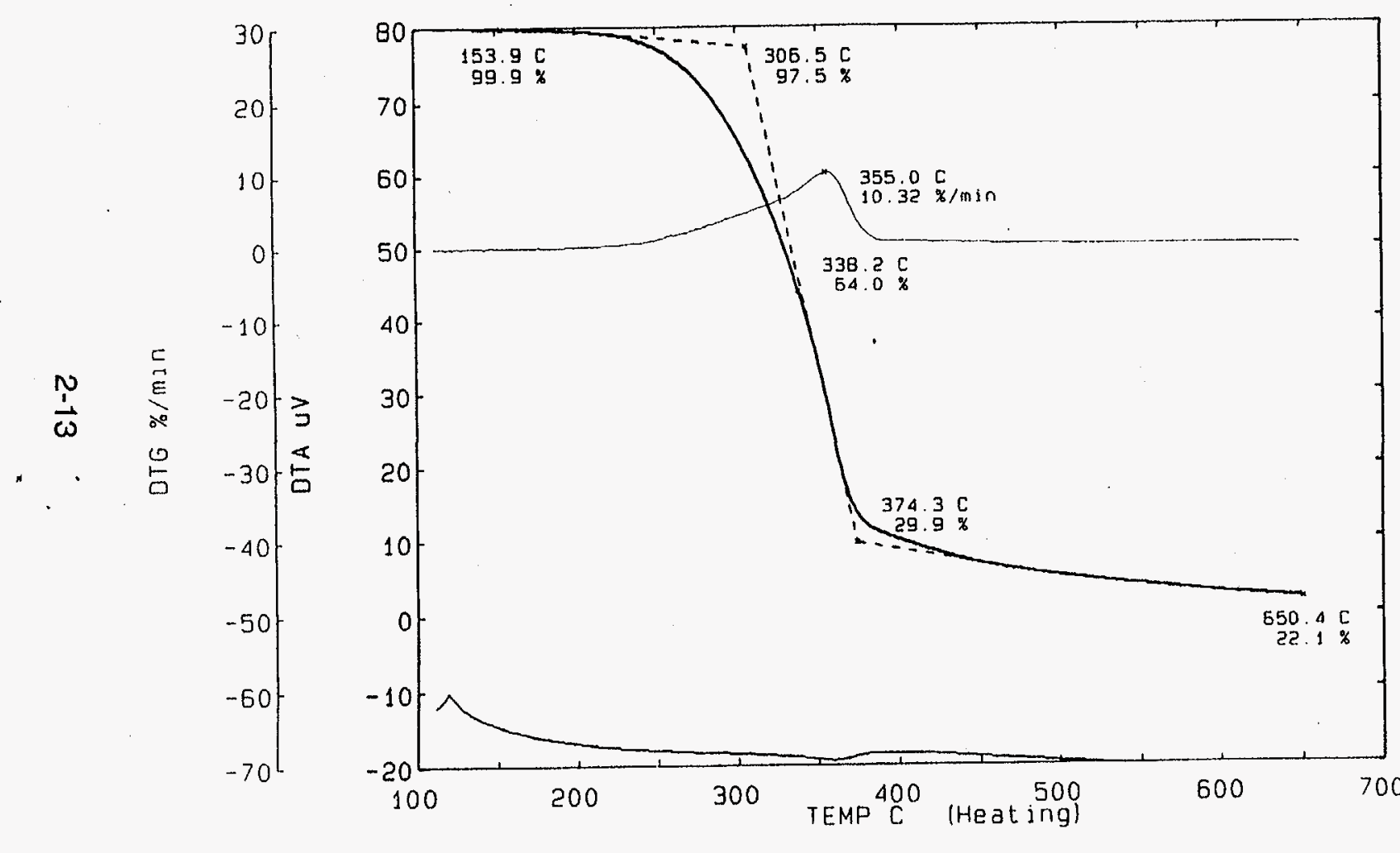

100

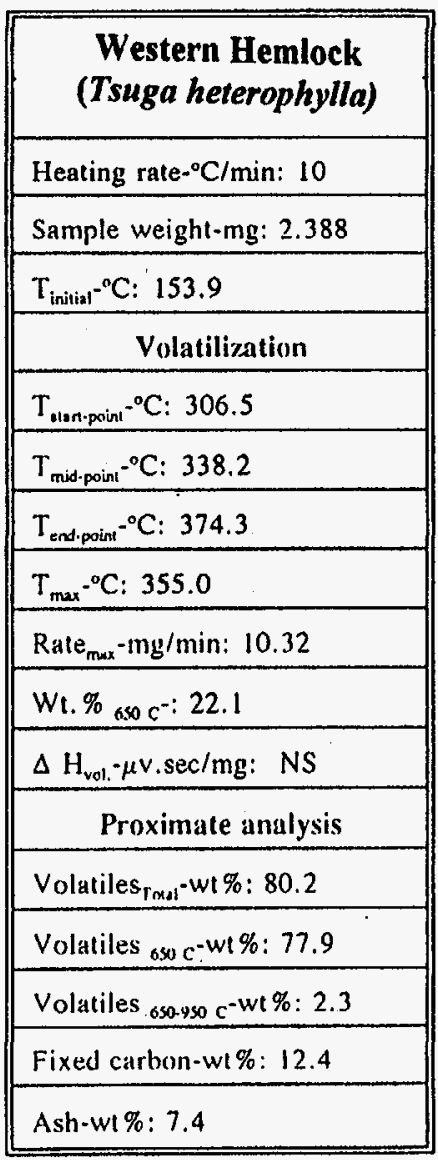



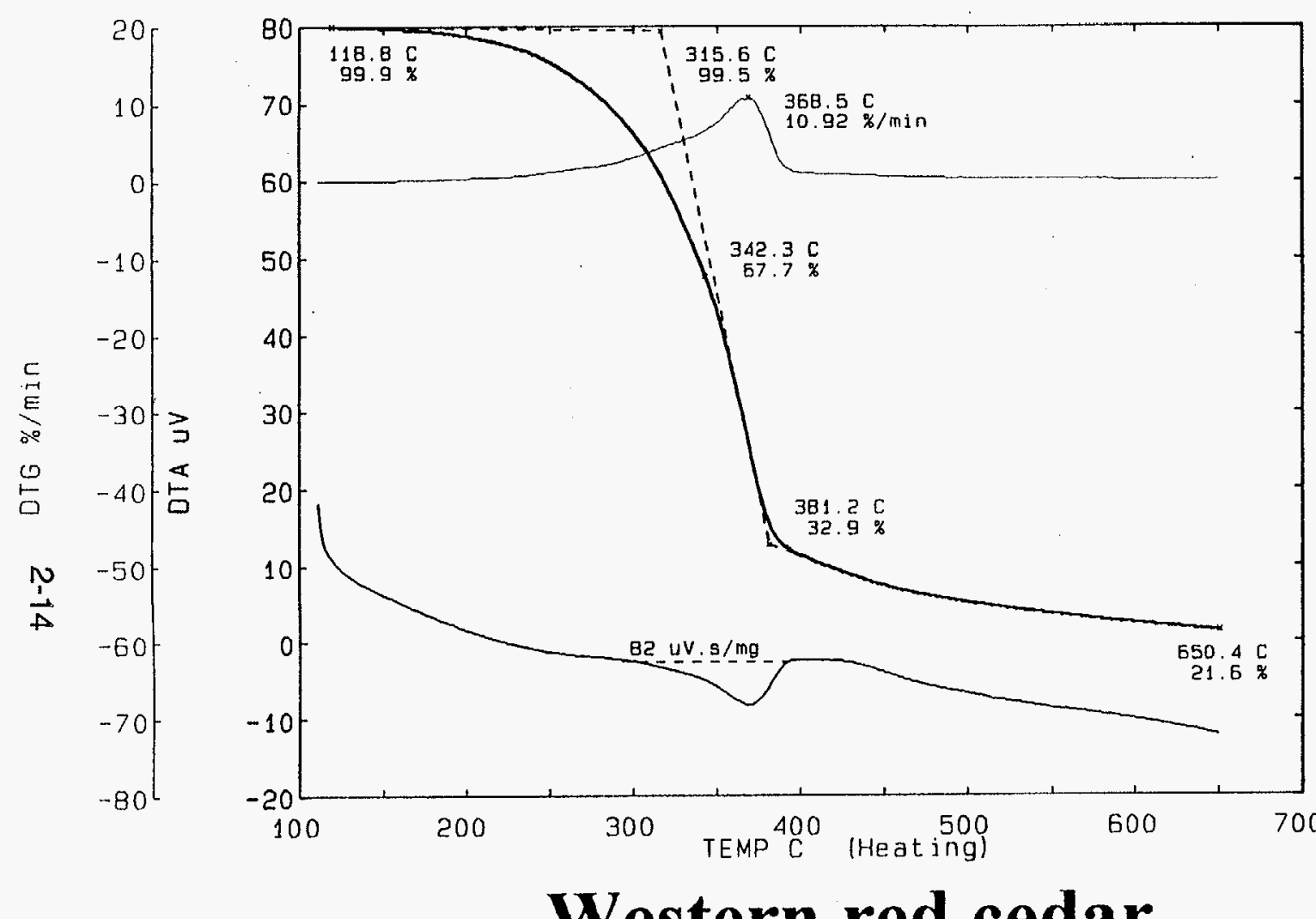

\begin{tabular}{|c|c|}
\hline 90 & $\begin{array}{l}\text { Western red cedar } \\
\text { (Tuja plicata) }\end{array}$ \\
\hline \multirow{2}{*}{80} & Heating rate- ${ }^{\circ} \mathrm{C} / \mathrm{min}: 10$ \\
\hline & Sample weight-mg: 1.535 \\
\hline 70 & $\mathrm{~T}_{\text {initial }}{ }^{-0} \mathrm{C}: 118.8$ \\
\hline \multirow{2}{*}{60} & Volatilization \\
\hline & $\mathrm{T}_{\text {statt-point }} \mathrm{-}^{\circ} \mathrm{C}: 315.6$ \\
\hline 50 & $\mathrm{~T}_{\text {mid-poim }}{ }^{\circ} \mathrm{C}: 342.3$ \\
\hline \multirow{2}{*}{$40 \stackrel{\circ}{\circ}$} & $\mathrm{T}_{\text {end-poin }}{ }^{\circ} \mathrm{C}: 381.2$ \\
\hline & $T_{\max }{ }^{\circ} \mathrm{C}: 368.5$ \\
\hline 30 & Rate $_{\max }-\mathrm{mg} / \mathrm{min}: 10.92$ \\
\hline \multirow{2}{*}{20} & $W_{t} . \%_{650} c^{-:}: 21.6$ \\
\hline & $\Delta H_{\mathrm{vol}}-\mu v . \mathrm{sec} / \mathrm{mg}: 82$ \\
\hline 10 & Proximate analysis \\
\hline \multirow{5}{*}{0} & Volatiles $_{\text {Tolal }}-\mathrm{wt} \%: 80.2$ \\
\hline & Volatiles $_{650} c^{-w t} \%: 78.4$ \\
\hline & Volatiles $_{650-950} \mathrm{c}^{-\mathrm{wt} \%: 1.8}$ \\
\hline & Fixed carbon-wt $\%: 18.0$ \\
\hline & Ash-wt \%: 1.8 \\
\hline
\end{tabular}



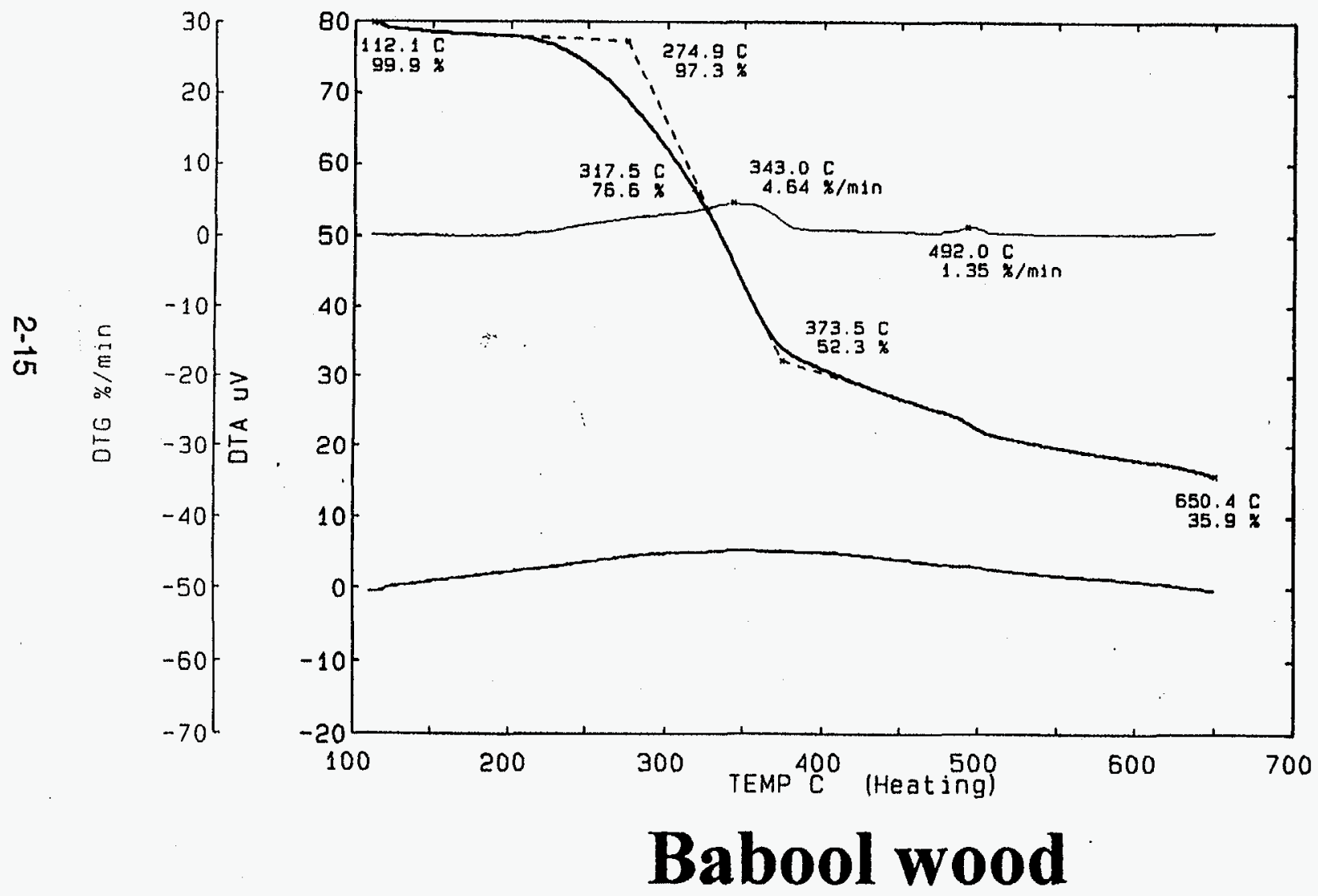

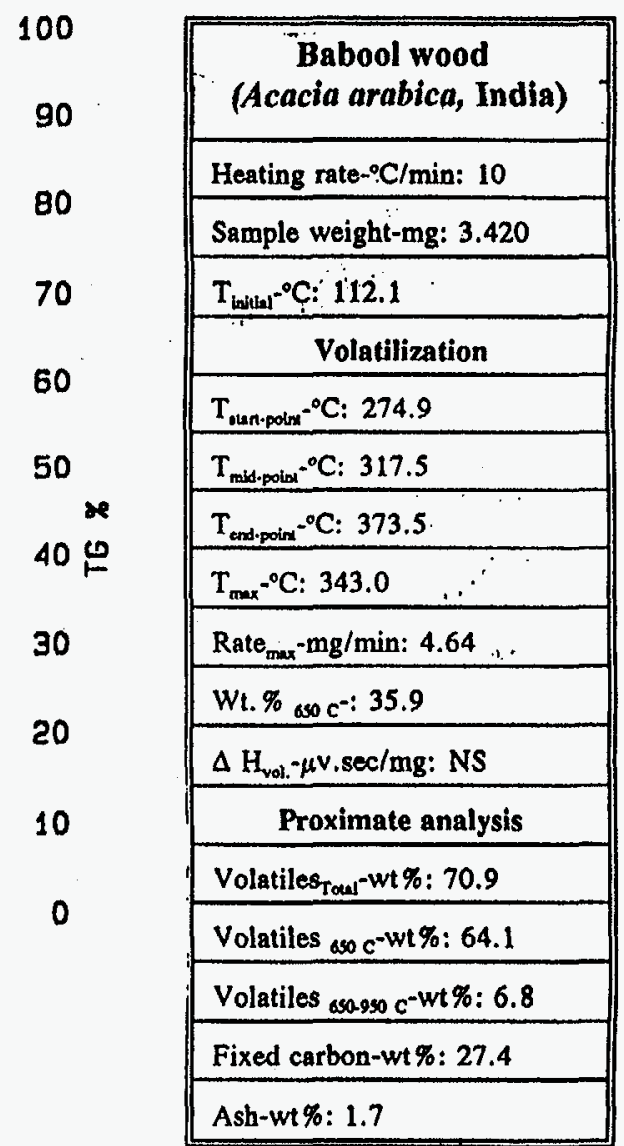

(Acacia arabica, India) 

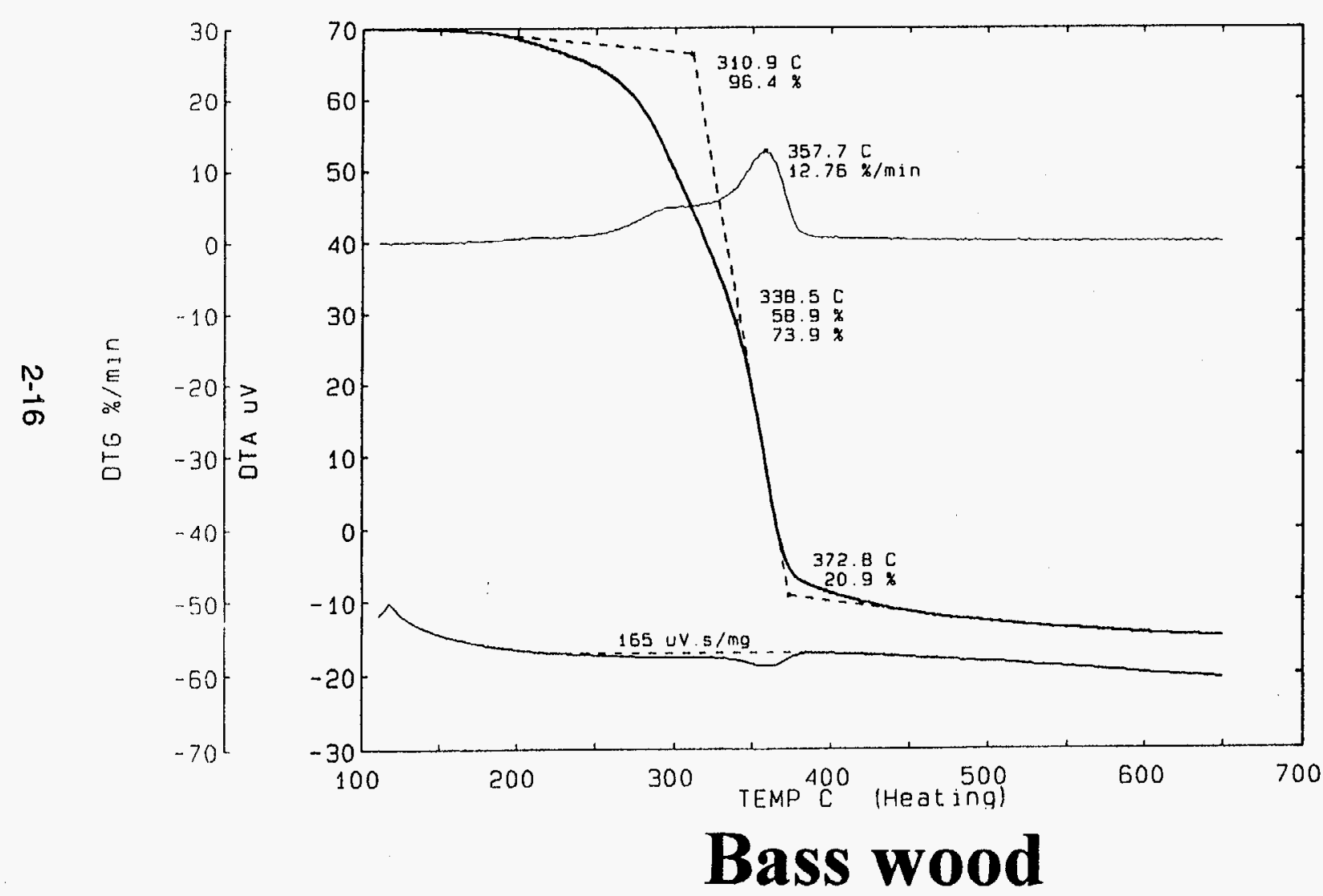

100

Bass wood (Tilia americana)

(Tilia americana) 

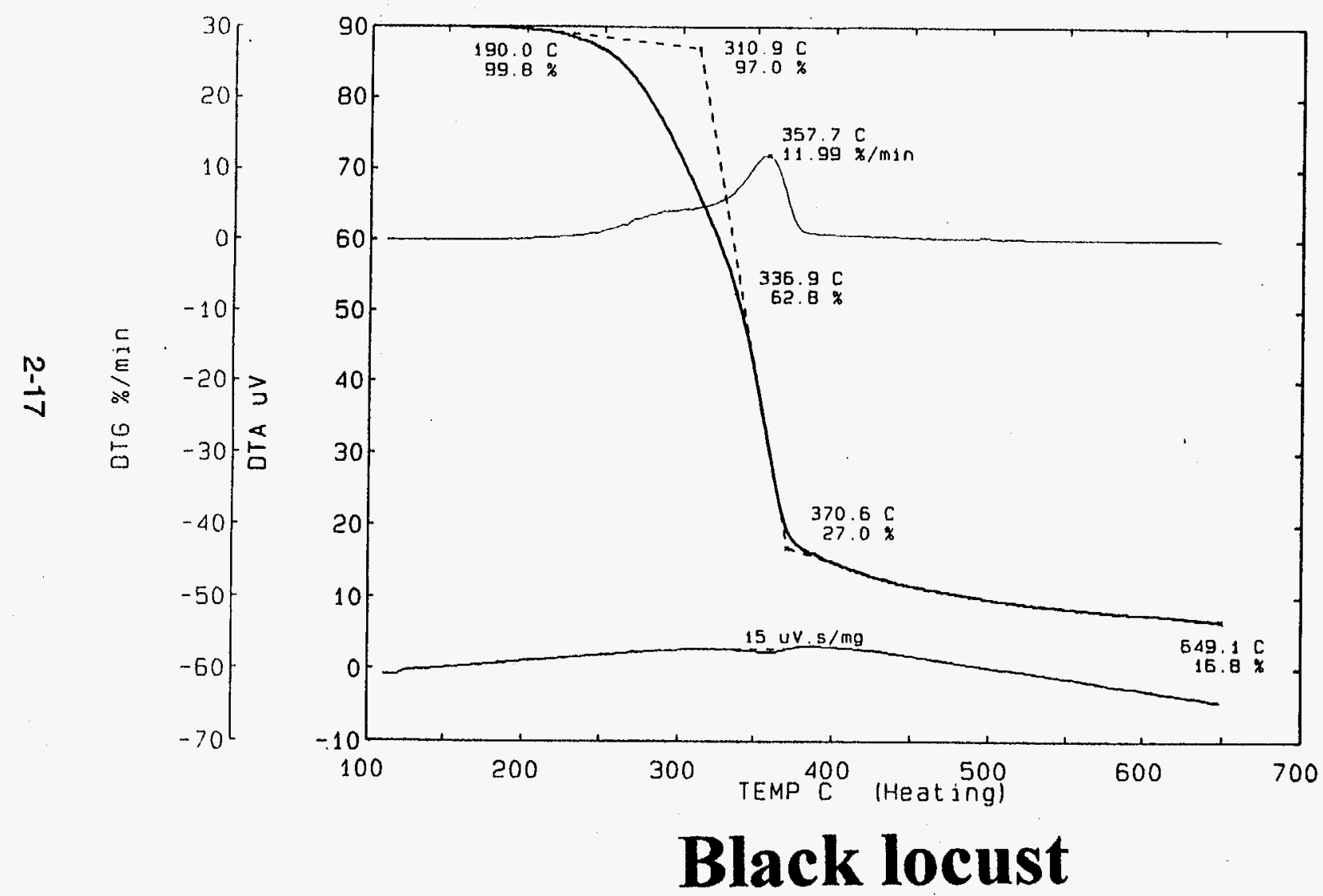

100
90
80
70
60
50
40
30
20
10
0

\section{(Robinia pseudoacacia)}

\begin{tabular}{|c|}
\hline $\begin{array}{c}\text { Black locust } \\
\text { (Robinia pseudoacacia) }\end{array}$ \\
\hline Heating rate- ${ }^{\circ} \mathrm{C} / \mathrm{min}: 10$ \\
\hline Sample weight-mg: 3.480 \\
\hline$T_{\text {iniliat }}{ }^{-0} \mathrm{C}: 190.0$ \\
\hline Volatilization \\
\hline$T_{\text {dun-point }}{ }^{\circ} \mathrm{C}: 310.9$ \\
\hline $\mathrm{T}_{\text {mid-point }}{ }^{-0} \mathrm{C}: \quad 336.9$ \\
\hline $\mathrm{T}_{\text {end-polm }}{ }^{-0} \mathrm{C}: 370.6$ \\
\hline$T_{\max }-^{\circ} \mathrm{C}: 357.7$ \\
\hline Rate $_{\operatorname{man}}-\mathrm{mg} / \mathrm{min}: 11.99$ \\
\hline Wt. \% $650 c^{-:}: 16.8$ \\
\hline 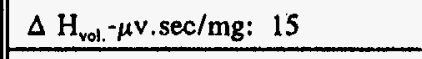 \\
\hline Proximate analysis \\
\hline Volatiles $_{\text {Tout }}-w 1 \%: 88.0$ \\
\hline Volatiles ${ }_{\text {aso }} c^{-w 1} \%: 83.2$ \\
\hline Volatiles $650-950 c^{-w t \%: ~} 4.8$ \\
\hline Fixed carbon-wt\%: 11.7 \\
\hline Ash-wt\%: 0.3 \\
\hline
\end{tabular}



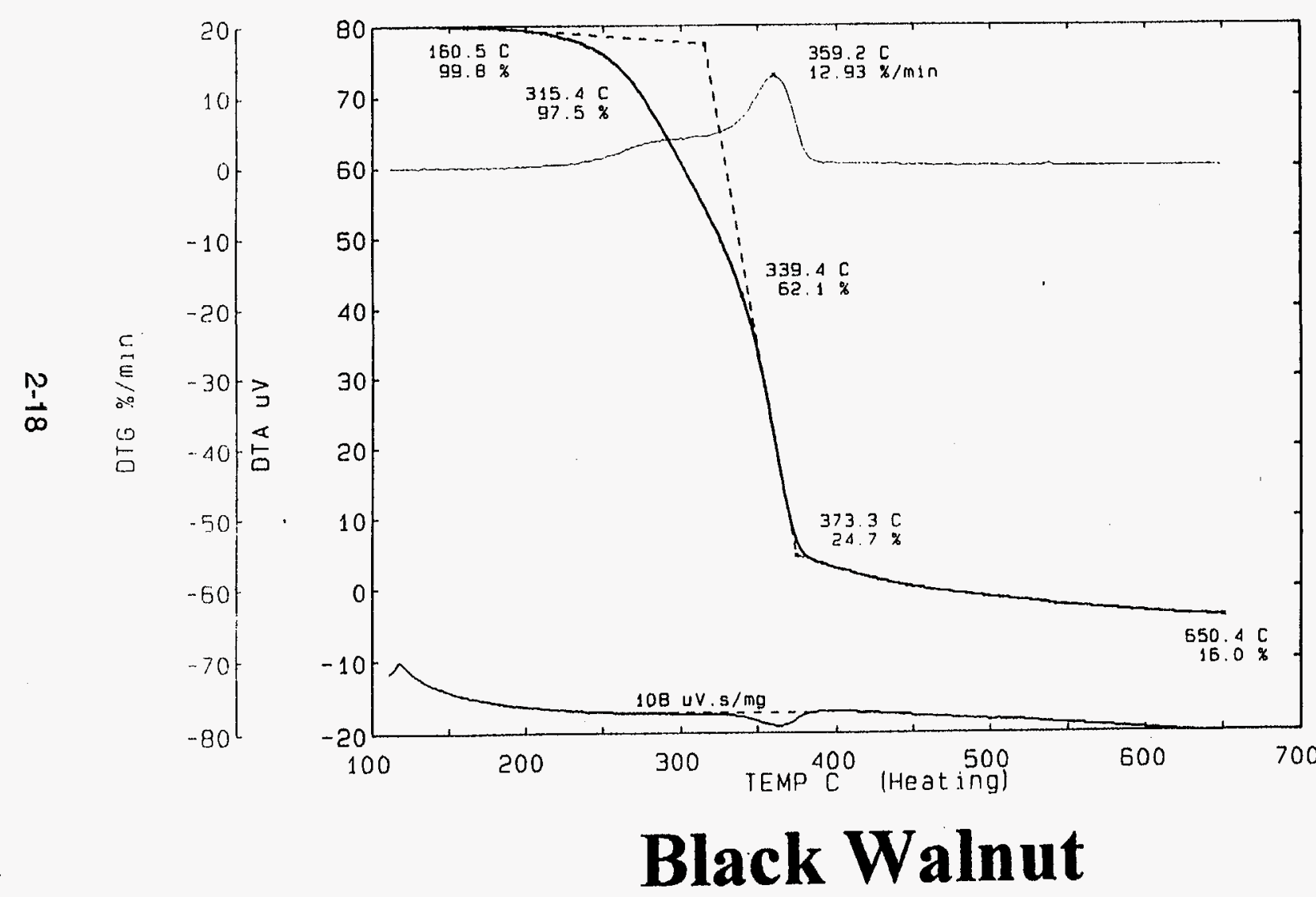

100

(Juglans nigra)

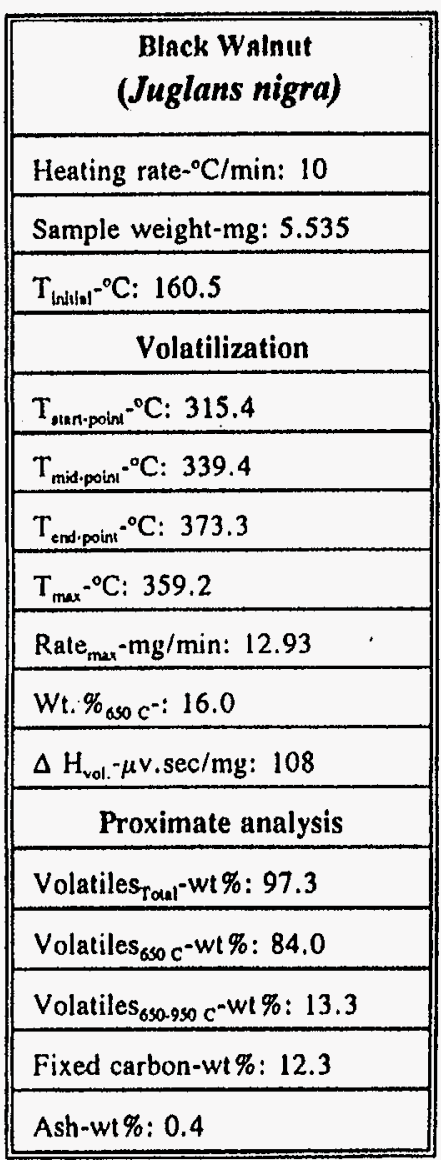




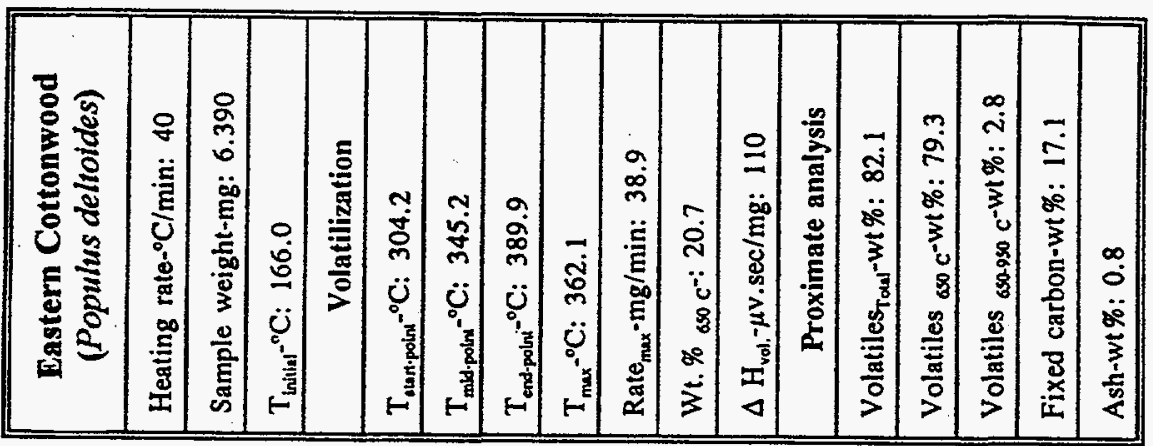

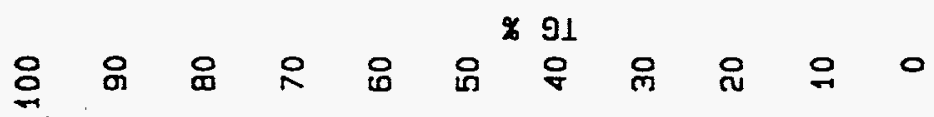

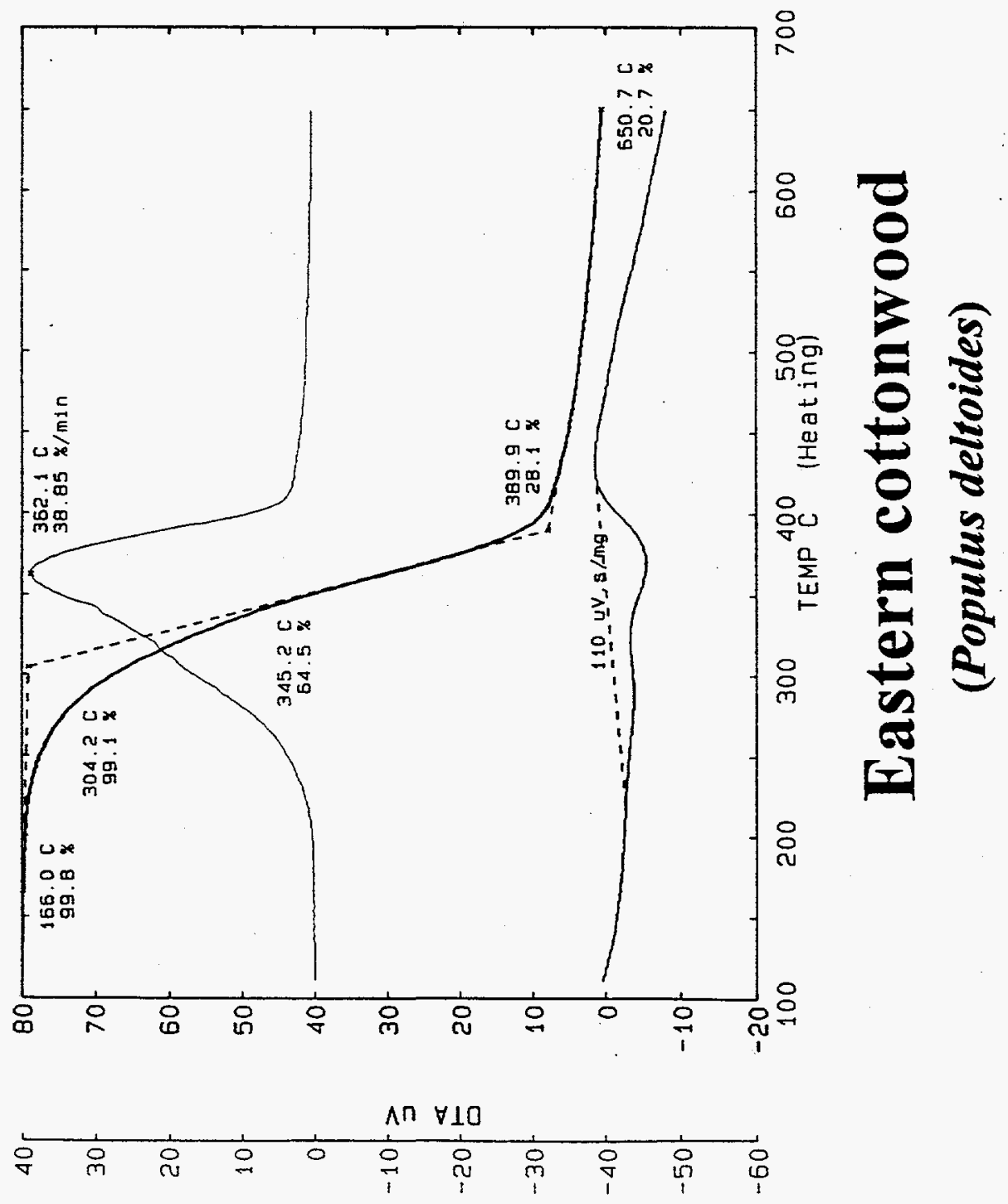

Utw/\% 910 

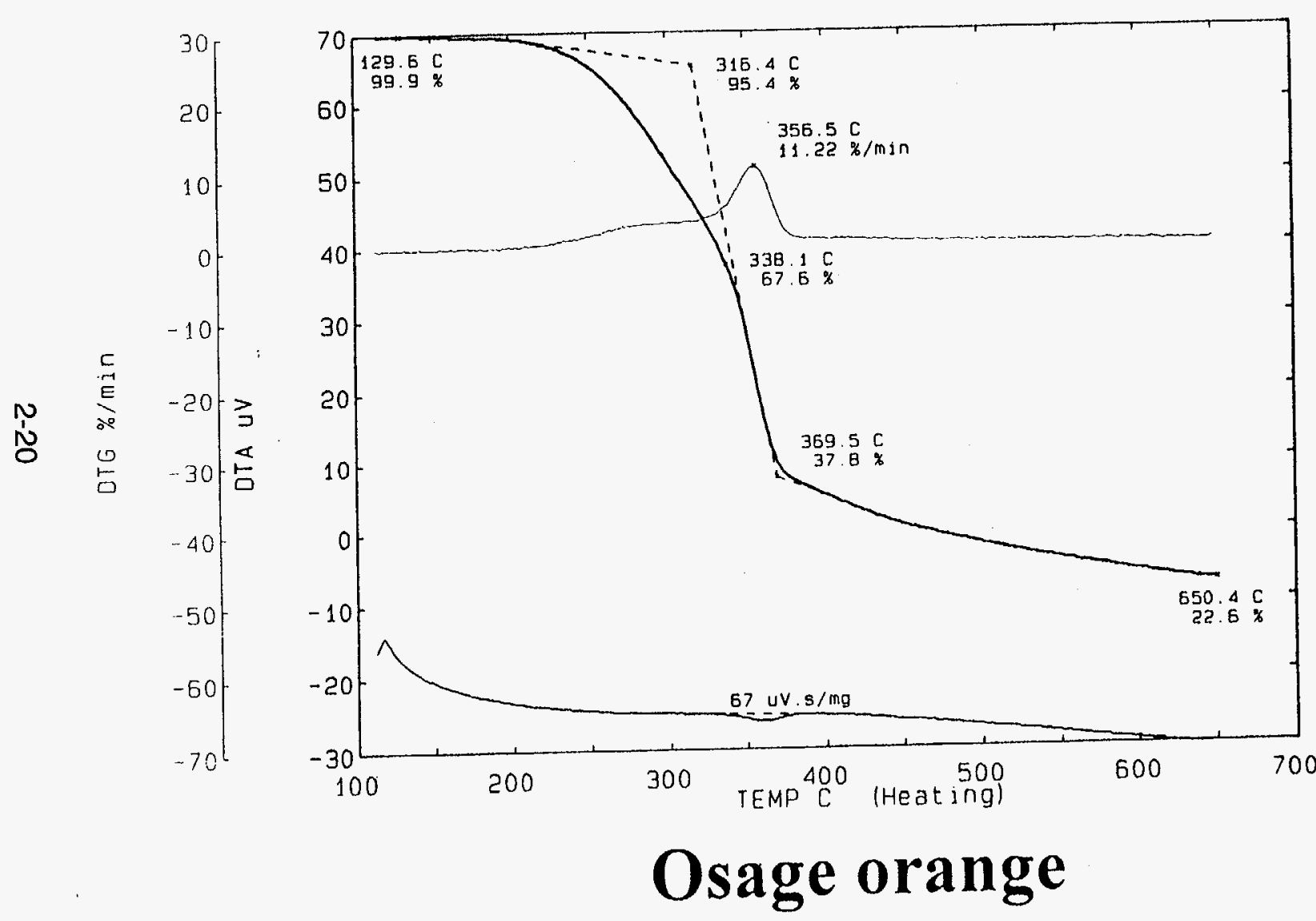

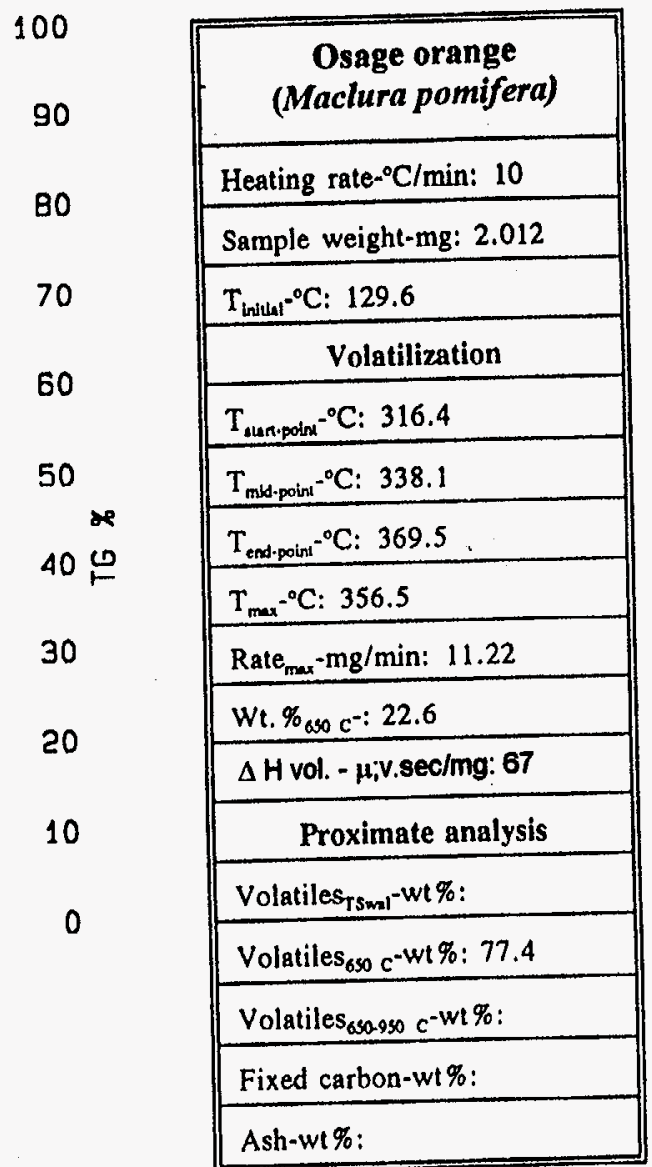

\section{(Maclura pomifera)}



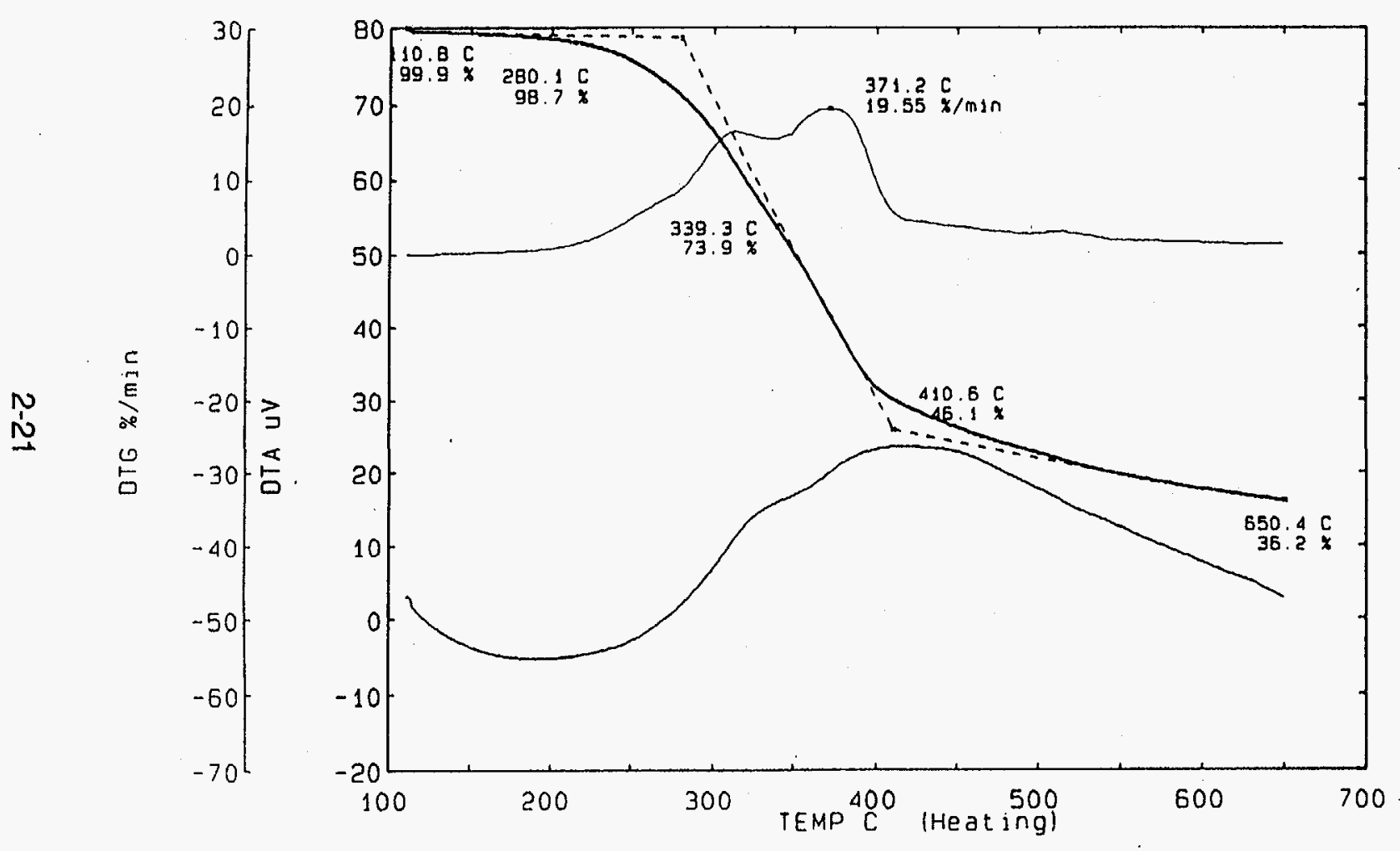

\begin{tabular}{|c|c|}
\hline 90 & $\begin{array}{l}\text { Red alder bark } \\
\text { (Alnus rubra) }\end{array}$ \\
\hline \multirow{2}{*}{80} & Heating rate. $.^{\circ} \mathrm{C} / \mathrm{min}: 10$ \\
\hline & Sample weight-mg: 11.01 \\
\hline 70 & $\mathrm{~T}_{\text {initiml }}{ }^{\circ} \mathrm{C}: 110.8$ \\
\hline \multirow{2}{*}{60} & Volatilization \\
\hline & $T_{\text {aun,pinin }}{ }^{\circ} \mathrm{C}: 280.1$ \\
\hline 50 & $T_{\text {mbl:polnt }}{ }^{-{ }^{-C} \mathrm{C}:} \quad 339.3$ \\
\hline \multirow[t]{2}{*}{$40 \stackrel{0}{\circ}$} & $T_{\text {exd:poin }}{ }^{\circ} \mathrm{C}: 410.6$ \\
\hline & $\mathrm{T}_{\operatorname{me}}{ }^{\circ} \mathrm{C}: 371.2$ \\
\hline 30 & Rate $_{\operatorname{mex}}-\mathrm{mg} / \mathrm{min}: 19.55$ \\
\hline \multirow{2}{*}{20} & $W_{t .} \%$ osoc: $: 36.2$ \\
\hline & $\Delta H_{v 01}-\mu v . s e c / m g: \quad N D$ \\
\hline 10 & Proximate analysis \\
\hline \multirow{5}{*}{0} & Volatiles $_{\text {Totol }}-w 1 \%: 70.3$ \\
\hline & Volatiles aso $c^{-w 1} \%: 63.8$ \\
\hline & Volatiles ${ }_{\text {wa. } 950} c^{-w 1} \%: 6.5$ \\
\hline & Fixed carbon-wi $\%: 26.2$ \\
\hline & Ash-wt\%: 3.5 \\
\hline
\end{tabular}

\section{Red alder bark}

(Alnus rubra) 


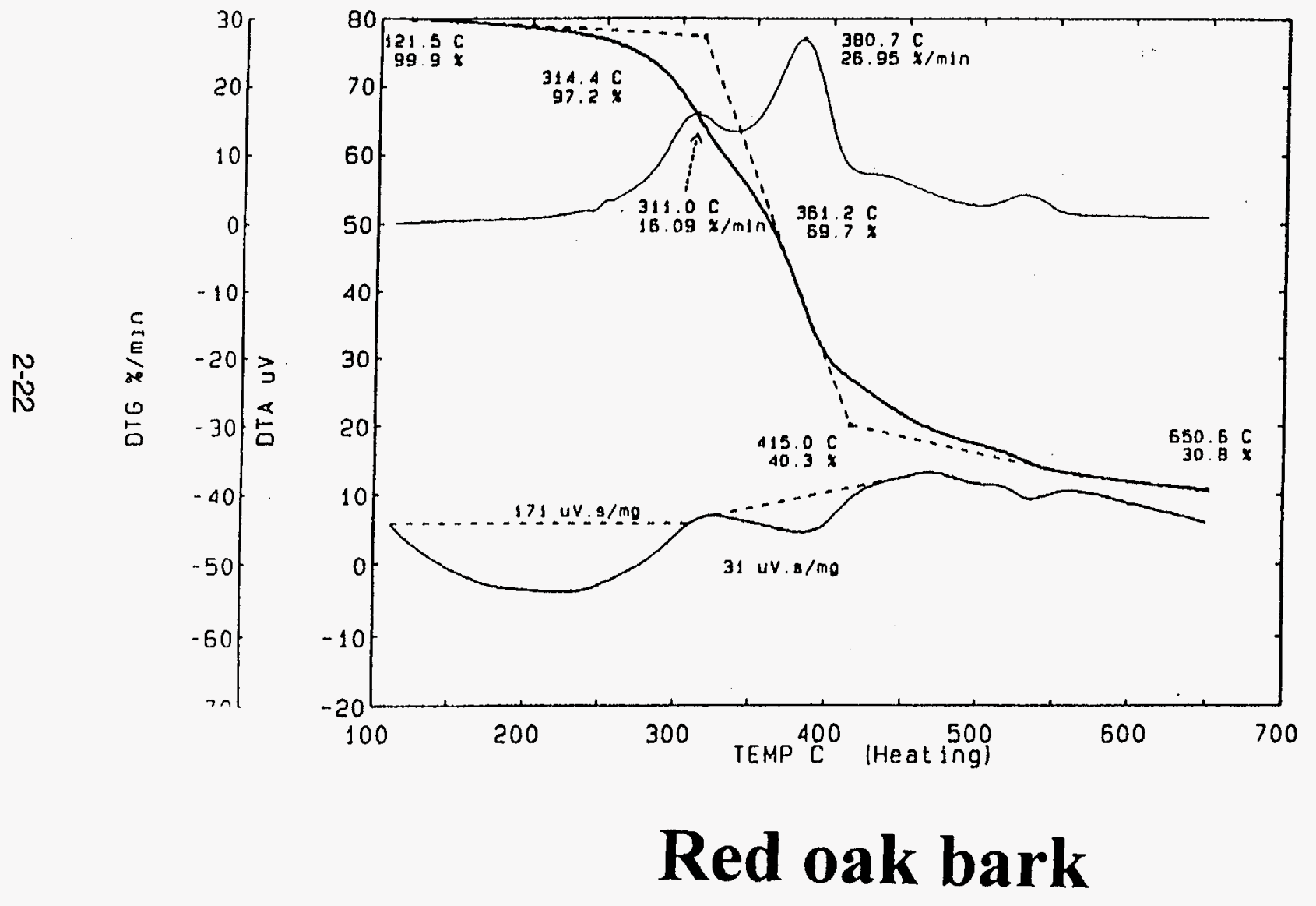

100
90
80
70
60
50
400
30
20
10
0

\begin{tabular}{|c|}
\hline $\begin{array}{c}\text { Red oak bark } \\
\text { (Quercus rubra) }\end{array}$ \\
\hline Heating rale. $\cdot{ }^{\circ} \mathrm{C} / \mathrm{min}: 10$ \\
\hline Sample weight-mg: 2.589 \\
\hline$T_{\text {welut } 10^{\circ}}{ }^{\circ} \mathrm{C}: 121.5$ \\
\hline Volatilization \\
\hline$T_{\text {ant.polex }}{ }^{*} \mathrm{C}: 314.4$ \\
\hline 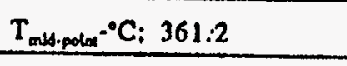 \\
\hline 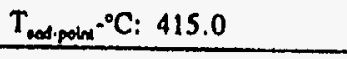 \\
\hline$T_{\text {max }}{ }^{\circ} \mathrm{C}: 380.7$ \\
\hline Rate $_{\max }-\mathrm{mg} / \mathrm{min}: 26.95$ \\
\hline W1. 90 a c: 30.8 \\
\hline$\Delta \mathrm{H}_{\mathrm{vol}}-\mu \mathrm{v}, \mathrm{sec} / \mathrm{mg}_{\mathrm{g}}: 171,31$ \\
\hline Proximale analysis \\
\hline Volatiles $_{\text {Tan }}$-wt $\%: 76.0$ \\
\hline Volatiles $\omega_{0} c^{* w 1 \%:} 69.2$ \\
\hline Volatiles ssa.sso $c^{-w 1 \%: 6.8}$ \\
\hline Fixed carbon-wt \%: 21.8 \\
\hline Ash-w1\%: 2.8 \\
\hline
\end{tabular}



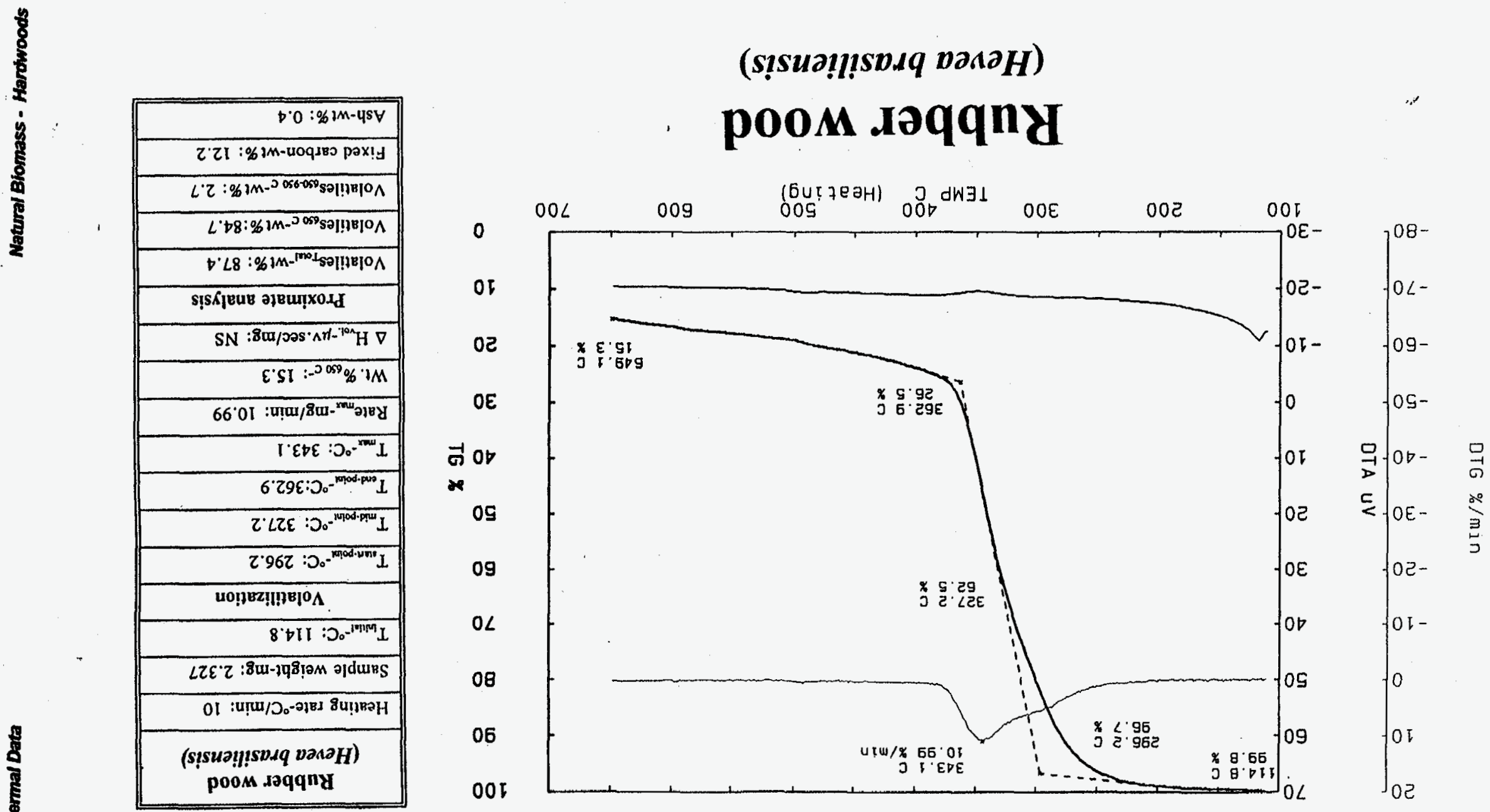

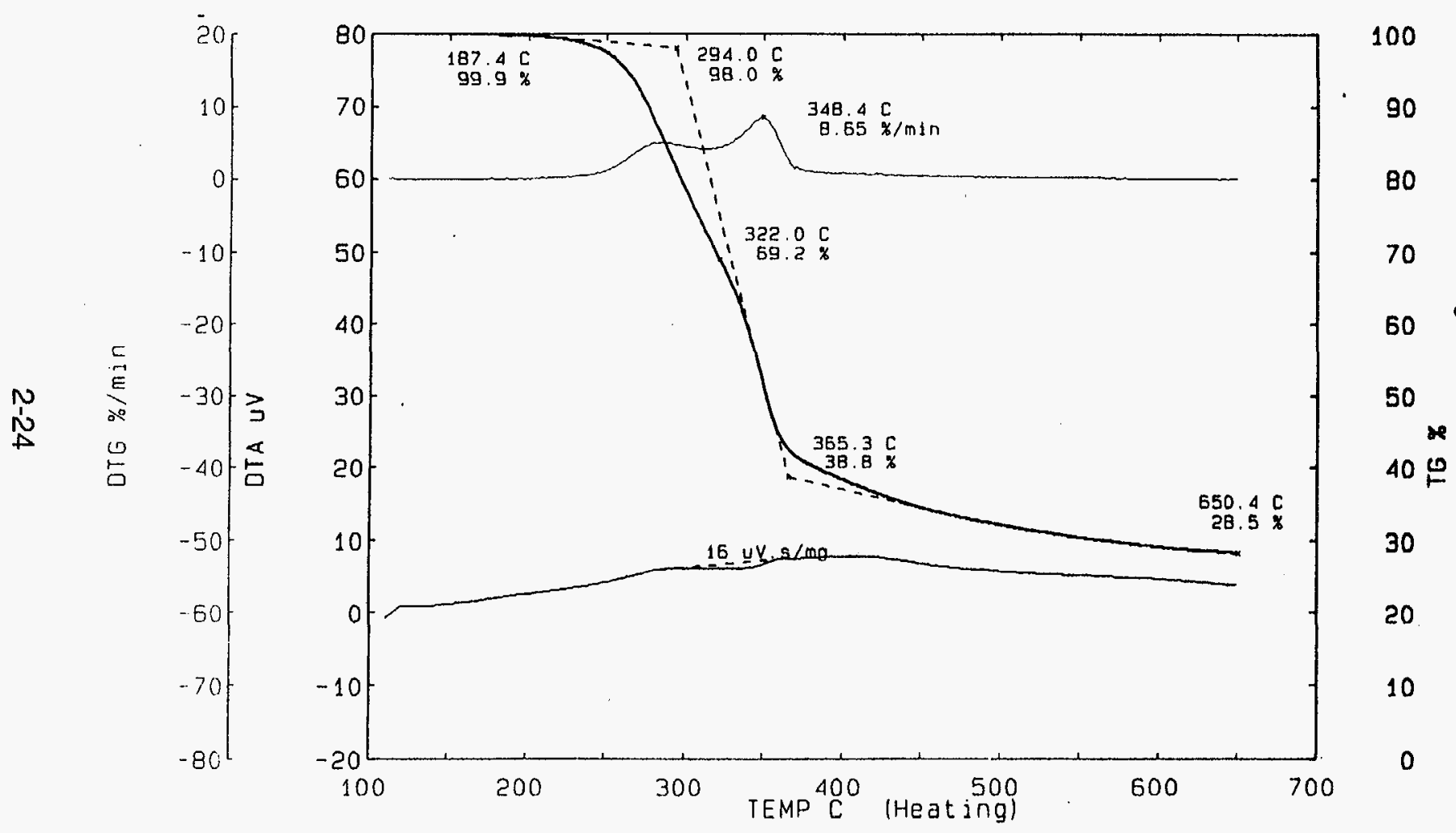

\section{Coconut shell}

\section{(Cocos nucifera)}

\begin{tabular}{|c|}
\hline $\begin{array}{l}\text { Coconut shell } \\
\text { (Cocos nucifera) }\end{array}$ \\
\hline Heating rate- $-^{\circ} \mathrm{C} / \mathrm{min}: 10$ \\
\hline Sample weight-mg: 9.69 \\
\hline$T_{\text {Lollat } 1}{ }^{-0} \mathrm{C}: 187.4$ \\
\hline Volatilization \\
\hline$T_{\text {ulan-polnt }}{ }^{-} \mathrm{C}: 294.0$ \\
\hline $\mathrm{T}_{m d-\text {-poim }}{ }^{-0} \mathrm{C}: 322.0$ \\
\hline $\mathrm{T}_{\text {end-point }}{ }^{\circ} \mathrm{C}: 365.3$ \\
\hline $\mathrm{T}_{\max } x^{\circ} \mathrm{C}: 348.4$ \\
\hline Rate $_{\operatorname{mux}}-\mathrm{mg} / \mathrm{min}: 8.65$ \\
\hline Wt. \% aso c $=28.5$ \\
\hline$\Delta \mathrm{H}_{\mathrm{vol} .}-\mu \mathrm{v} . \mathrm{sec} / \mathrm{mg}: 16$ \\
\hline Proximate analysis \\
\hline Volatiles $_{\text {Toul1 }}-w t \%: 73.5$ \\
\hline Volatiles coso $c^{-w 1 \%: 71.5}$ \\
\hline Volatiles $950 \mathrm{c}^{-w t} \%: 2.0$ \\
\hline Fixed carbon-wt\%: 25.8 \\
\hline Ash-wi\%: 0.7 \\
\hline
\end{tabular}



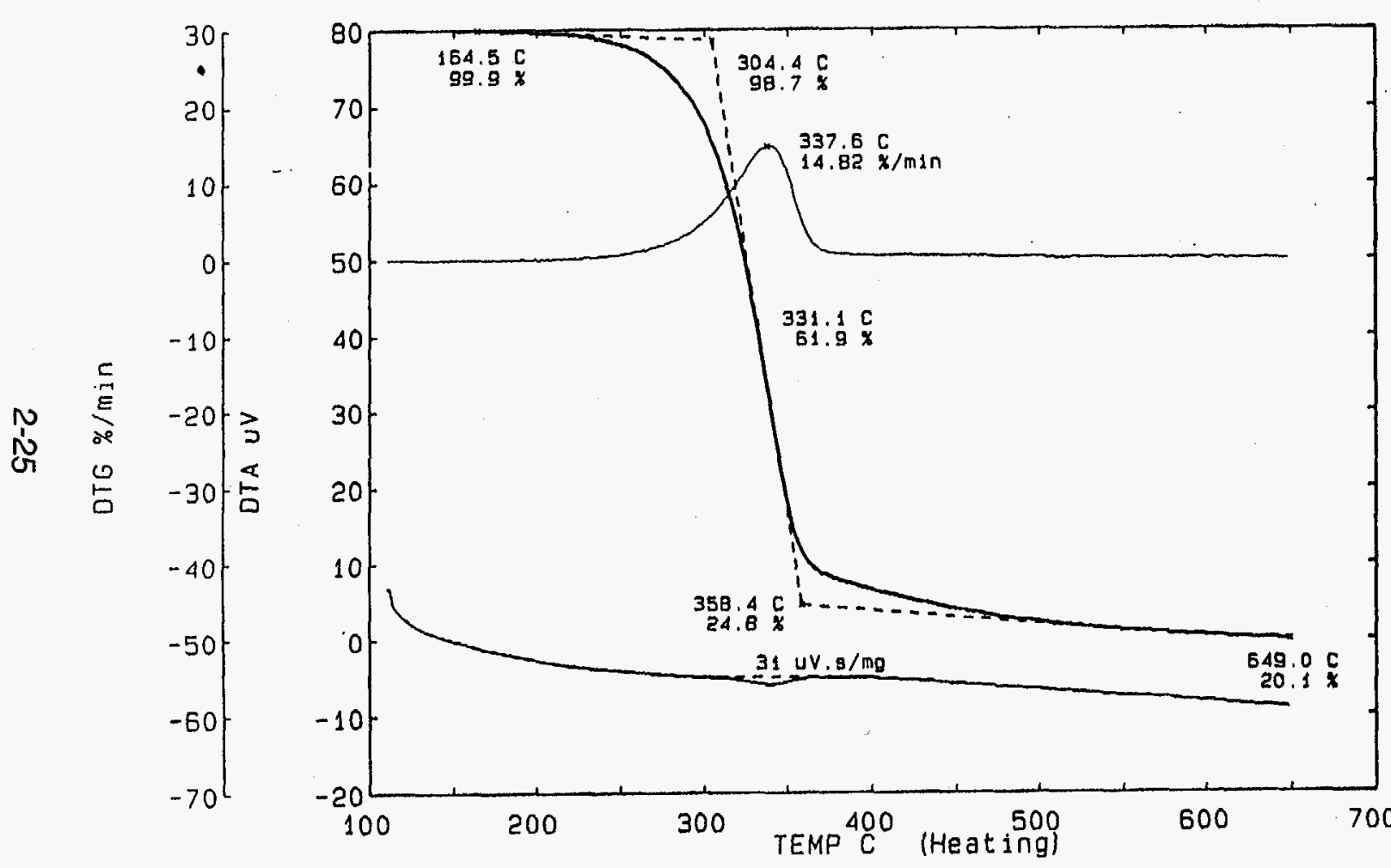

100

90

BO

70

60

50

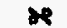

$40 \cong$

30

20

10

0

Cotton

(Gossypium)

\begin{tabular}{|c|}
\hline $\begin{array}{l}\text { Cotton } \\
\text { (Gossypium) }\end{array}$ \\
\hline Heating rate- ${ }^{\circ} \mathrm{C} / \mathrm{min}: 10$ \\
\hline Sample weight-mg: 4.884 \\
\hline $\mathrm{T}_{\text {mitter }}{ }^{\circ} \mathrm{C}: 164.5$ \\
\hline Volatilization \\
\hline$T_{\text {aun-polat }}{ }^{-} \mathrm{C}: \quad 304.4$ \\
\hline$T_{\text {mid.polax }}{ }^{-0} \mathrm{C}: 331.1$ \\
\hline$T_{\text {endppexat }}{ }^{-} \mathrm{C}: 358.4$ \\
\hline$T_{\max }{ }^{-0} \mathrm{C}: 337.6$ \\
\hline Rate $_{\max }-\mathrm{mg} / \mathrm{min}: 14.82$ \\
\hline Wt. \% ${ }_{\text {aso }}^{\circ} c^{-:}: 20.1$ \\
\hline$\Delta \mathrm{H}_{\mathrm{vol} .} \cdot \mu \mathrm{v} . \mathrm{sec} / \mathrm{mg}: 31$ \\
\hline Proximate analysis \\
\hline Volatiles $_{\text {Tous }}-w t \%: 82.4$ \\
\hline Volatiles $\operatorname{sos} c^{-w t} \%: 79.9$ \\
\hline Volatiles $.000 .950 c^{-w 1} \%: 2.5$ \\
\hline Fixed carbon-wt\%: 15.8 \\
\hline Ash-wt\%: 1.8 \\
\hline
\end{tabular}



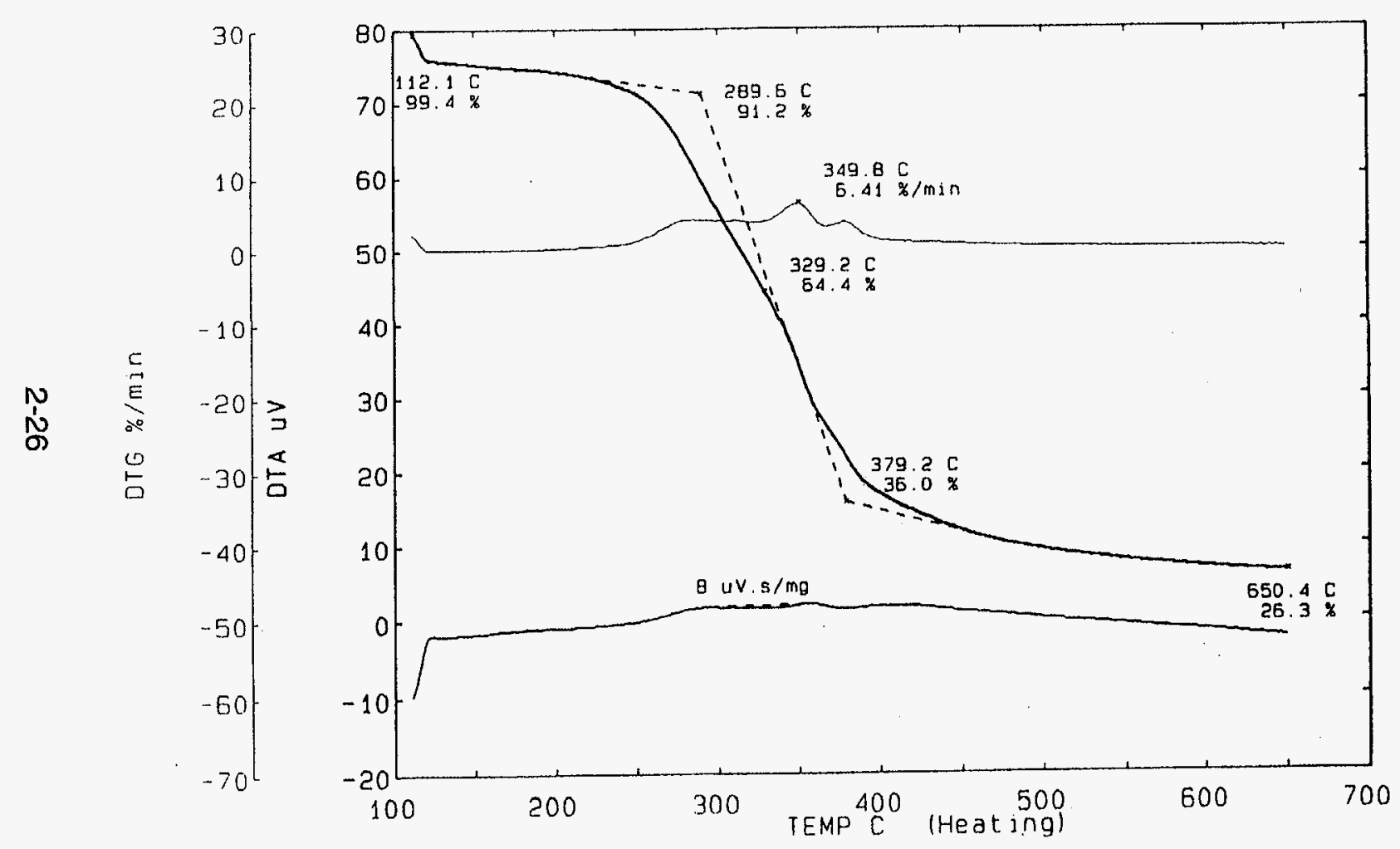

100

\begin{tabular}{|c|}
\hline $\begin{array}{c}\text { Peach seed } \\
\text { (Prunus persica) }\end{array}$ \\
\hline Heating rate- ${ }^{\circ} \mathrm{C} / \mathrm{min}: 10$ \\
\hline Sample weight-mg: 9.13 \\
\hline $\mathrm{T}_{\text {Inditit }}^{-{ }^{-} \mathrm{C}: 112.1}$ \\
\hline Volatilization \\
\hline $\mathrm{T}_{\text {aun-posio }}{ }^{-\mathrm{C}} \mathrm{C}: 289.6$ \\
\hline$T_{\text {mid.polin }}{ }^{-0} \mathrm{C}: 329.2$ \\
\hline$T_{\text {end.poite }}{ }^{\circ} \mathrm{C}: 379.2$ \\
\hline $\mathrm{T}_{\max }{ }^{-0} \mathrm{C}: 349.8$ \\
\hline Rate $_{\text {max }}-\mathrm{mg} / \mathrm{min}: 6.41$ \\
\hline$W_{t} . \%_{\text {Gso } c^{-}: 26.3}$ \\
\hline 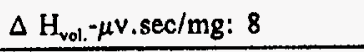 \\
\hline Proximate analysis \\
\hline Volatiles $_{\text {Toul }}-w 1 \%: 74.8$ \\
\hline 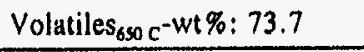 \\
\hline Volatiles $_{s 00.950} c^{-w 1 \%: 1.1}$ \\
\hline Fixed carbon-wt $\%: 24.2$ \\
\hline Ash-wt \%: 1.0 \\
\hline
\end{tabular}



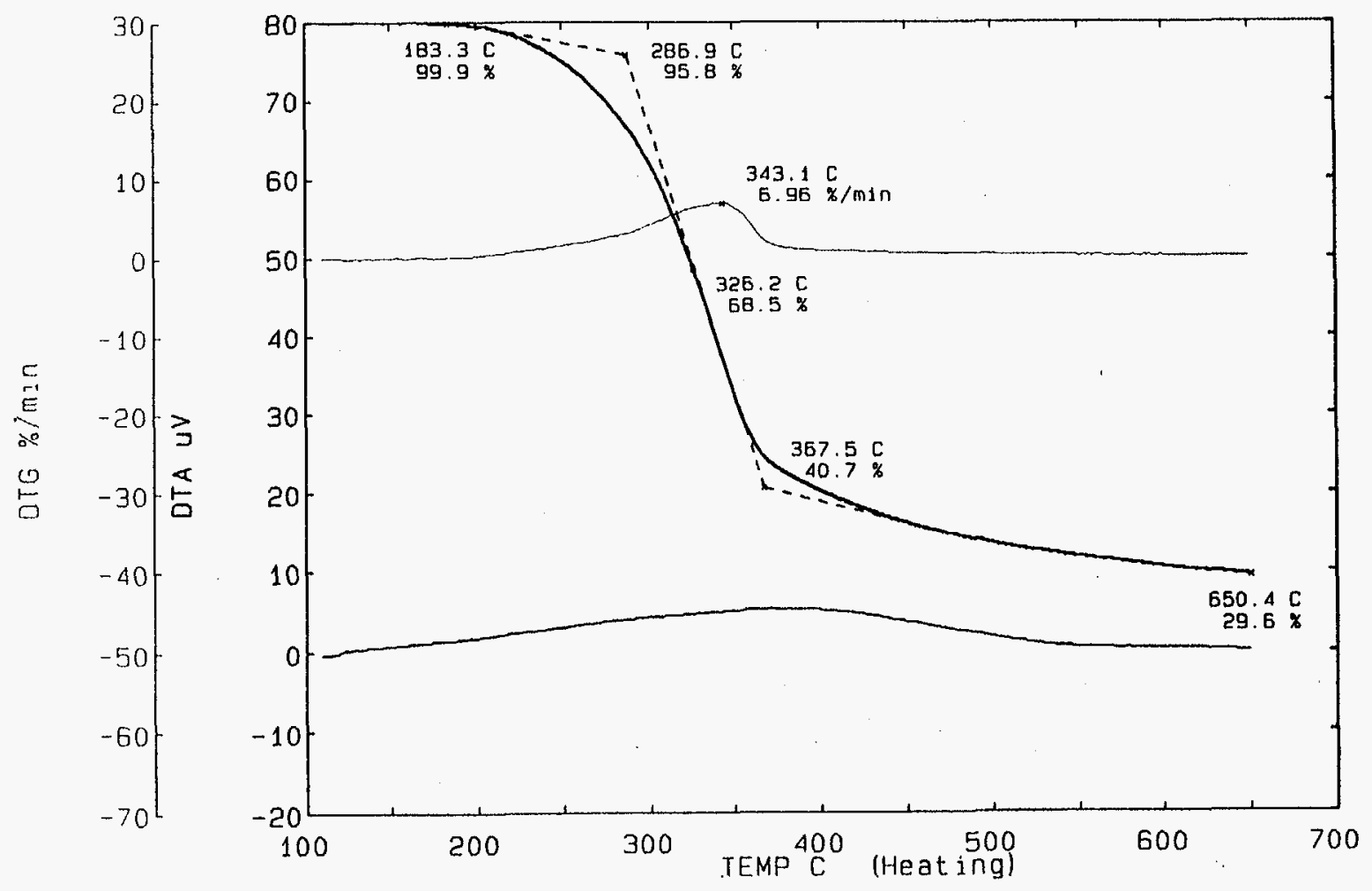

Peanut shell

(Arachis hypogaea)

\begin{tabular}{|c|c|}
\hline 90 & $\begin{array}{c}\text { Peanut shell } \\
\text { (Arachis hypogaea) }\end{array}$ \\
\hline \multirow{2}{*}{80} & Heating rate- ${ }^{\circ} \mathrm{C} / \mathrm{min}: 10$ \\
\hline & Sample weight-mg: 4.718 \\
\hline 70 & $\mathrm{~T}_{\text {intiliat }}{ }^{\circ} \mathrm{C}: 183.3$ \\
\hline \multirow{2}{*}{60} & Volatilization \\
\hline & $T_{\text {nan-polnt }}{ }^{-} \mathrm{C}: 286.9$ \\
\hline \multirow{3}{*}{$\begin{array}{l}50 \\
40 \stackrel{0}{0}\end{array}$} & $T_{\text {mid-point }}{ }^{\circ} \mathrm{C}: \quad 326.2$ \\
\hline & $\mathrm{T}_{\text {end point }}{ }^{-{ }^{\circ} \mathrm{C}:} 367.5$ \\
\hline & $T_{\max }{ }^{-0} \mathrm{C}: 343.1$ \\
\hline 30 & Rate $_{\text {max }}-\mathrm{mg} / \mathrm{min}: 6.96$ \\
\hline \multirow{2}{*}{20} & Wt. $\%_{650 c^{-:}: 29.6}$ \\
\hline & $\Delta \mathrm{H}_{\mathrm{vol}}-\mu \mathrm{v} \cdot \mathrm{sec} / \mathrm{mg}: \mathrm{NS}$ \\
\hline \multirow[t]{2}{*}{10} & Proximate analysis \\
\hline & Volatiles $_{\text {Toul }}-w t \%: 73.8$ \\
\hline \multirow{4}{*}{0} & Volatiles $_{6 S 0} c^{-w 1} \%: 70.4$ \\
\hline & Volatiles $_{\text {sagasso }} c^{-w 1 \%: 3.4}$ \\
\hline & Fixed carbon-wt\%: 24.4 \\
\hline & Ash-wt $\%: 1.8$ \\
\hline
\end{tabular}



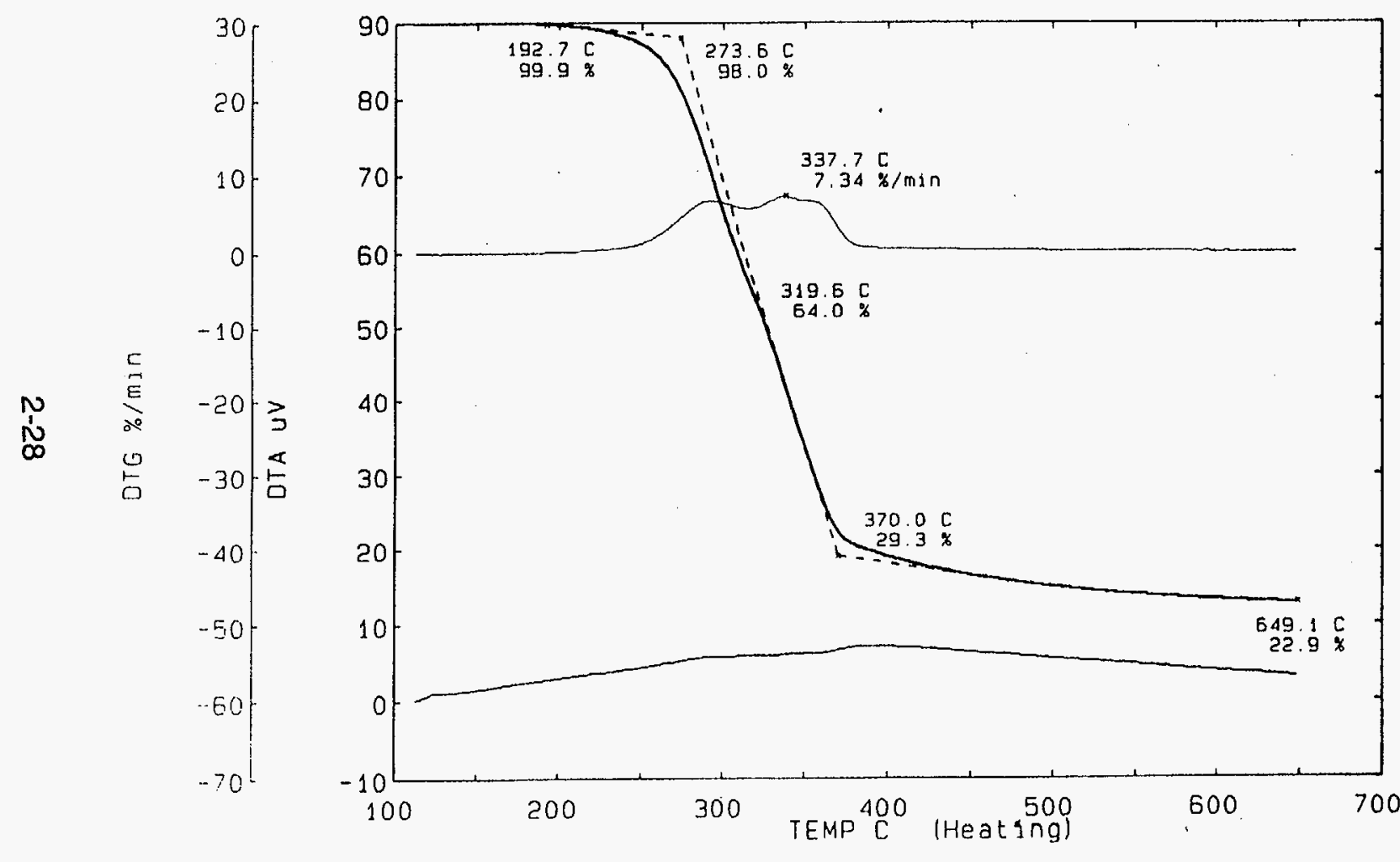

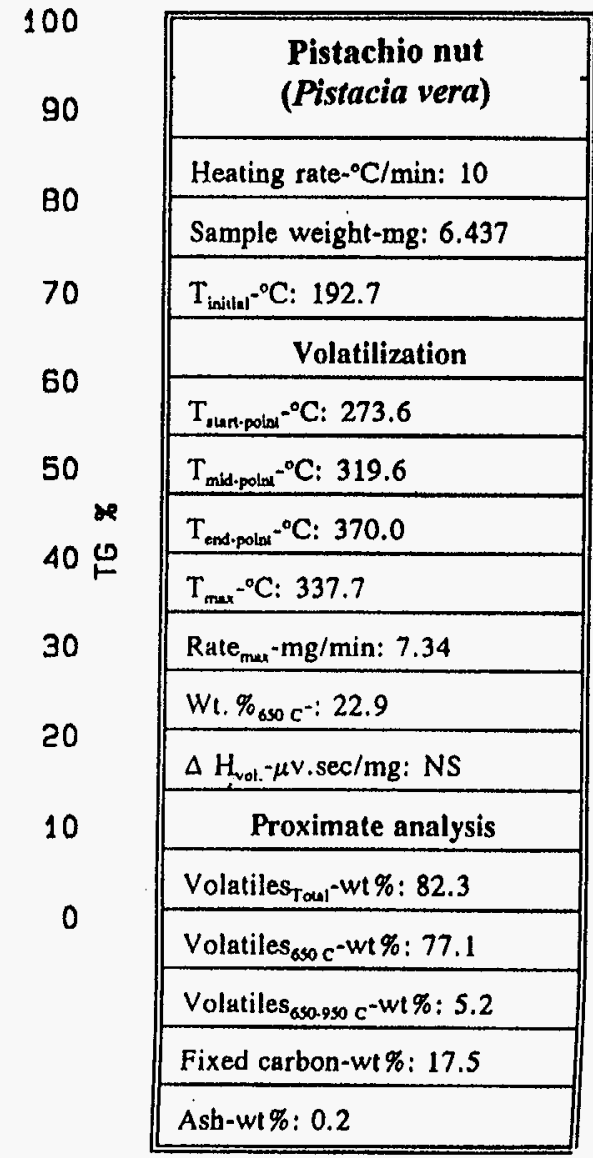

\section{Pistachio nut}

(Pistacia vera) 

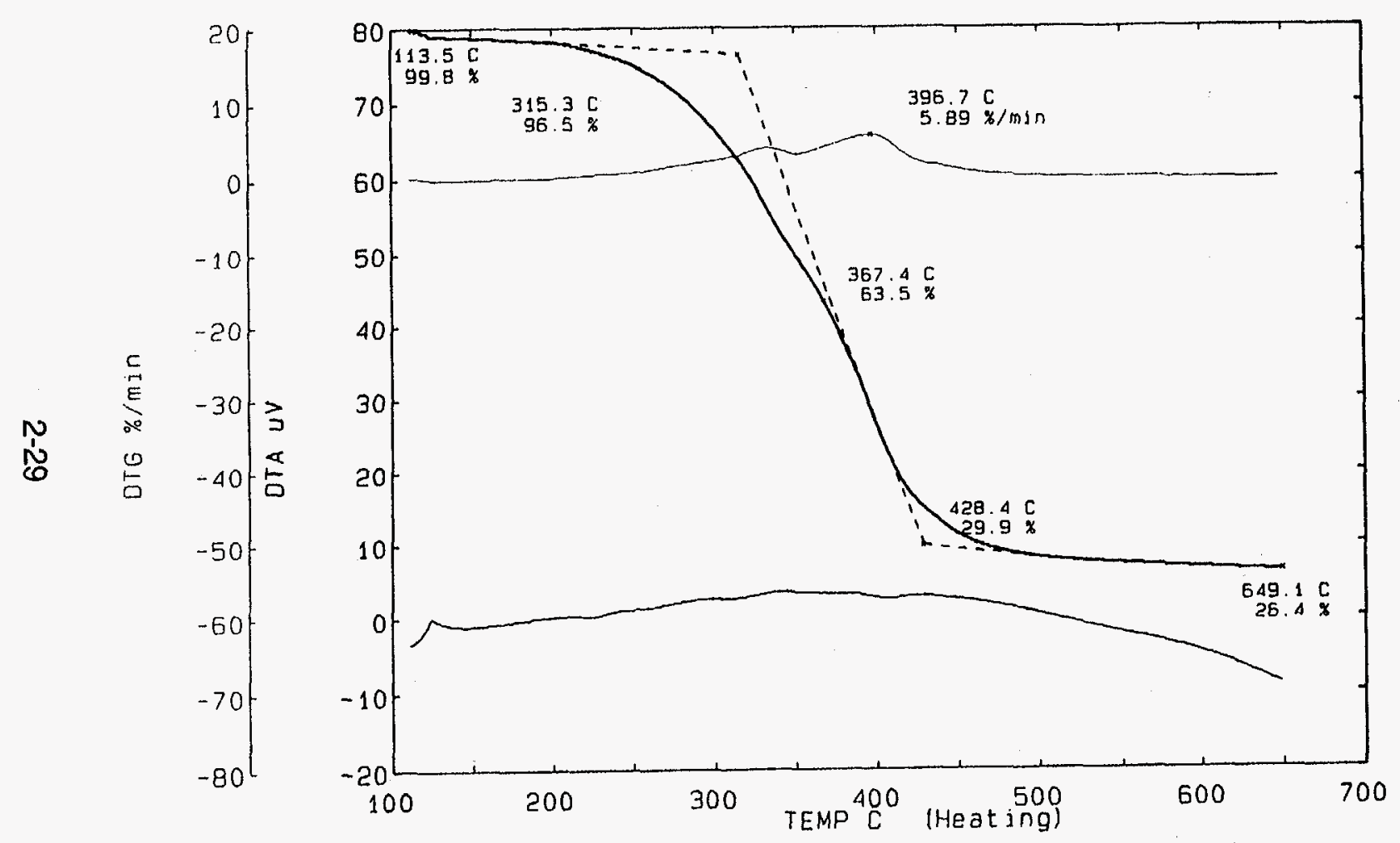

\begin{tabular}{|c|c|}
\hline 100 & $\begin{array}{l}\text { Pumpkin seed } \\
\text { (Cucurbita pepo) }\end{array}$ \\
\hline \multirow{2}{*}{80} & Heating rate $.^{\circ} \mathrm{C} / \mathrm{min}: 10$ \\
\hline & Sample weight-mg: 14.59 \\
\hline \multirow[t]{2}{*}{70} & $\mathrm{~T}_{\text {inititat }}{ }^{-\mathrm{C}} \mathrm{C}: 113.5$ \\
\hline & Volatilization \\
\hline 60 & $T_{\text {oun-poisx }}{ }^{-} \mathrm{C}: 315.3$ \\
\hline \multirow{3}{*}{$\begin{array}{l}50 \\
400\end{array}$} & $\mathrm{~T}_{\text {mid.point }}{ }^{\circ} \mathrm{C}: \mathrm{B}=7.4$ \\
\hline & $\mathrm{T}_{\text {ent-polm }}{ }^{-{ }^{\circ} \mathrm{C}:} \mathbf{4 2 8 . 4}$ \\
\hline & $T_{\max }{ }^{\circ} \mathrm{C}: 396.7$ \\
\hline \multirow[t]{2}{*}{30} & Rate $_{\max }-\mathrm{mg} / \mathrm{min}: 5.89$ \\
\hline & Wt. $\%_{650} c^{-:}: 26.4$ \\
\hline 20 & $\Delta \mathrm{H}_{\mathrm{vol}}-\mu \mathrm{v} . \mathrm{sec} / \mathrm{mg}: \mathrm{NS}$ \\
\hline \multirow[t]{2}{*}{10} & Proximate analysis \\
\hline & Volatiles $_{\text {Toul }}-w t \%: 92.9$ \\
\hline \multirow[t]{4}{*}{0} & Volatiles $_{\Delta s 0 c-w t \%: 73.6}$ \\
\hline & Volatiles $_{500.950} c^{-w t} \%: 19.3$ \\
\hline & Fixed carbon-wt\%: 3.3 \\
\hline & Ash-wt\%: 3.8 \\
\hline
\end{tabular}

\section{Pumpkin seed}

(Cucurbita pepo) 

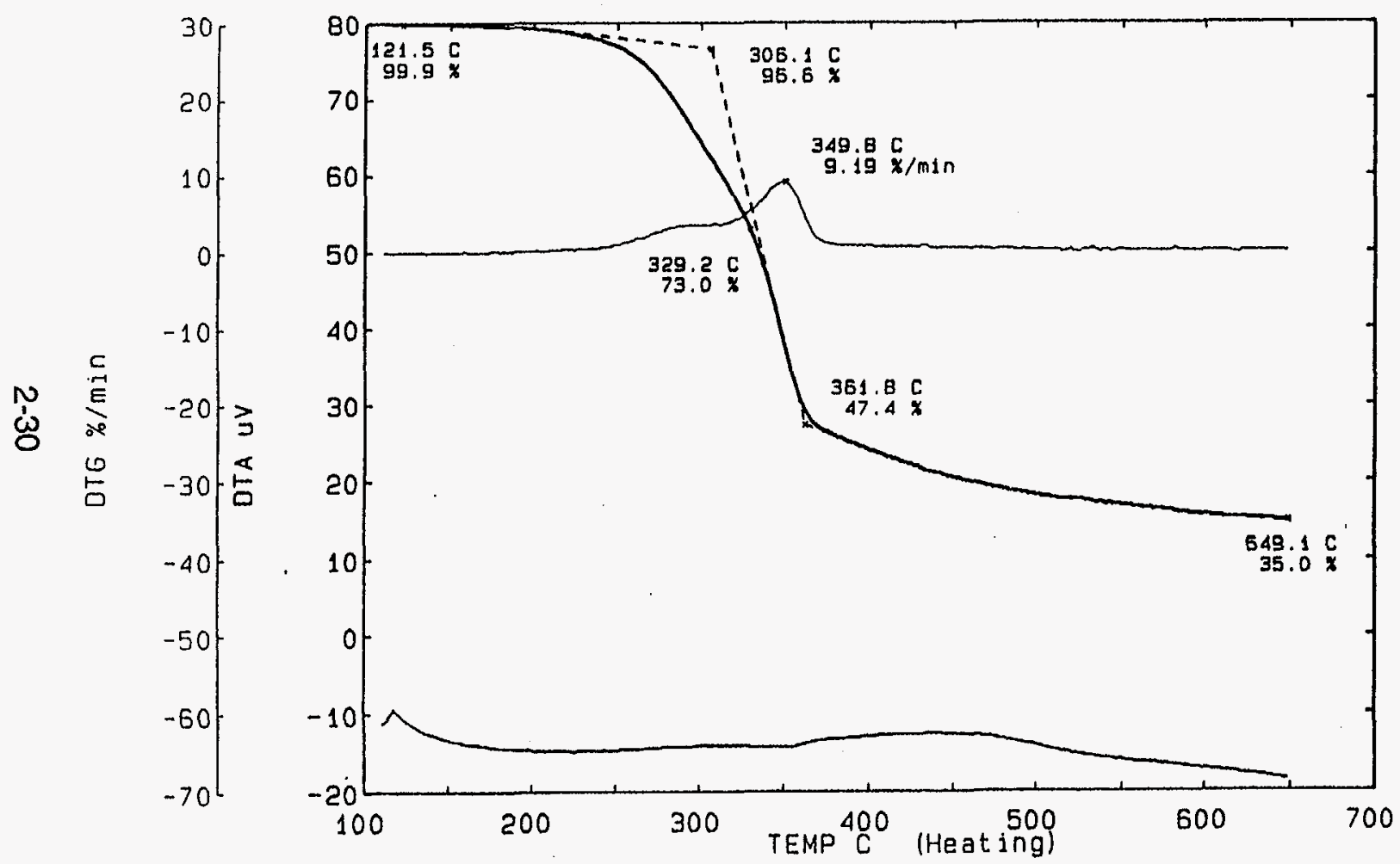

\begin{tabular}{|c|c|}
\hline 90 & $\begin{array}{c}\text { Rice hull } \\
\text { (Oryza sativa) }\end{array}$ \\
\hline \multirow{2}{*}{80} & Heating rate- ${ }^{\circ} \mathrm{C} / \mathrm{min}: 10$ \\
\hline & Sample weight-mg: $\mathbf{3 . 8 2 6}$ \\
\hline 70 & $\mathrm{~T}_{\text {bellut }}{ }^{-0} \mathrm{C}: 121.5$ \\
\hline \multirow{2}{*}{60} & Volatilization \\
\hline & $T_{\text {tunn-posian }}{ }^{-} \mathrm{C}: 306.1$ \\
\hline \multirow{3}{*}{$\begin{array}{l}50 \\
40 \stackrel{0}{5}\end{array}$} & $T_{\text {md-potax }}{ }^{-0} \mathrm{C}: 329.2$ \\
\hline & $\mathrm{T}_{\text {edd.polatx }}{ }^{\circ} \mathrm{C}: 361.8$ \\
\hline & $T_{\operatorname{mex}}{ }^{-0} \mathrm{C}: 349.8$ \\
\hline 30 & Rato $_{\operatorname{mex}}-\mathrm{mg} / \min : 9.19$ \\
\hline \multirow{2}{*}{20} & $W_{t} . \%_{050} c^{-:}: 35.0$ \\
\hline & $\Delta \mathrm{H}_{\text {olo }}-\mu \mathrm{v} . \mathrm{sec} / \mathrm{mg}: \mathrm{NS}$ \\
\hline 10 & Proximate analysis \\
\hline \multirow{5}{*}{0} & Volatiles $_{\text {Tous }}-w 1 \%: 67.7$ \\
\hline & Volatiles $_{\omega s 0} c^{-w t} \%: 65$ \\
\hline & Volatiles $_{\text {S5a. } 950} c^{-w t} \%: 2.7$ \\
\hline & Fixed carbon-wt\%: 14.1 \\
\hline & Ash-wt\%: 18.2 \\
\hline
\end{tabular}

Rice hull

(Oryza sativa) 

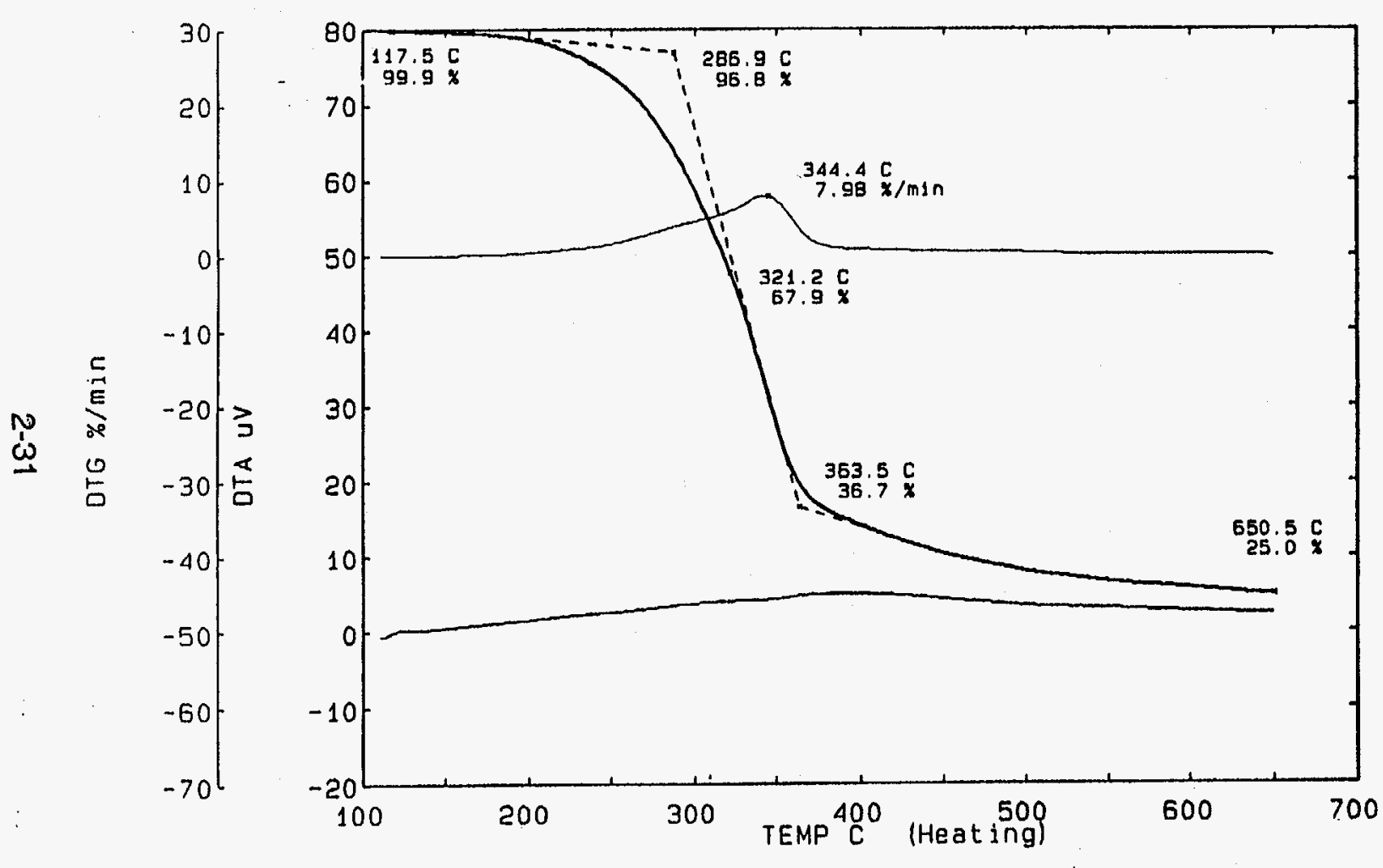

Sericea lespedeza

(Sericea lespedeza cuneata var. serala)
100

\begin{tabular}{|c|}
\hline $\begin{array}{l}\text { Sericen lespedeza } \\
\text { (Lespedeza cuneata ver. } \\
\text { serala) }\end{array}$ \\
\hline Heating rate-- ${ }^{\circ} \mathrm{C} / \mathrm{min}: 10$ \\
\hline Samplo woight-mg: 6.029 \\
\hline$T_{\text {tolwat }}{ }^{-0} \mathrm{C}: 117.5$ \\
\hline Volatilization \\
\hline $\mathrm{T}_{\text {mantpodsex }}{ }^{-\mathrm{C}:}: 286.9$ \\
\hline $\mathrm{T}_{\text {matpolax }}{ }^{\circ \mathrm{C}:} 321.2$ \\
\hline $\mathrm{T}_{\text {datax }}{ }^{\circ} \mathrm{C}: 363.5$ \\
\hline$T_{\max }{ }^{\circ} \mathrm{C}: 344.4$ \\
\hline $\mathrm{Rato}_{\max }-\mathrm{mg} / \mathrm{min}: 8.0$ \\
\hline Wt. $\%$ aso $c^{-:}: 25$ \\
\hline$\Delta H_{\text {vol. }}-\mu v . s e c / m g: N D$ \\
\hline Proximate analysis \\
\hline Volatiles $_{\text {Tous }}-\mathrm{wt} \%: 76.5$ \\
\hline Volatiles $_{\Delta s 0} c^{-w t} \%: 75.0$ \\
\hline Volatiles $_{s a, 0 s 0} c^{-w t} \%: 1.5$ \\
\hline Fixed carbon-wt\%: 21.6 \\
\hline Ash-wt\%: 1.9 \\
\hline
\end{tabular}



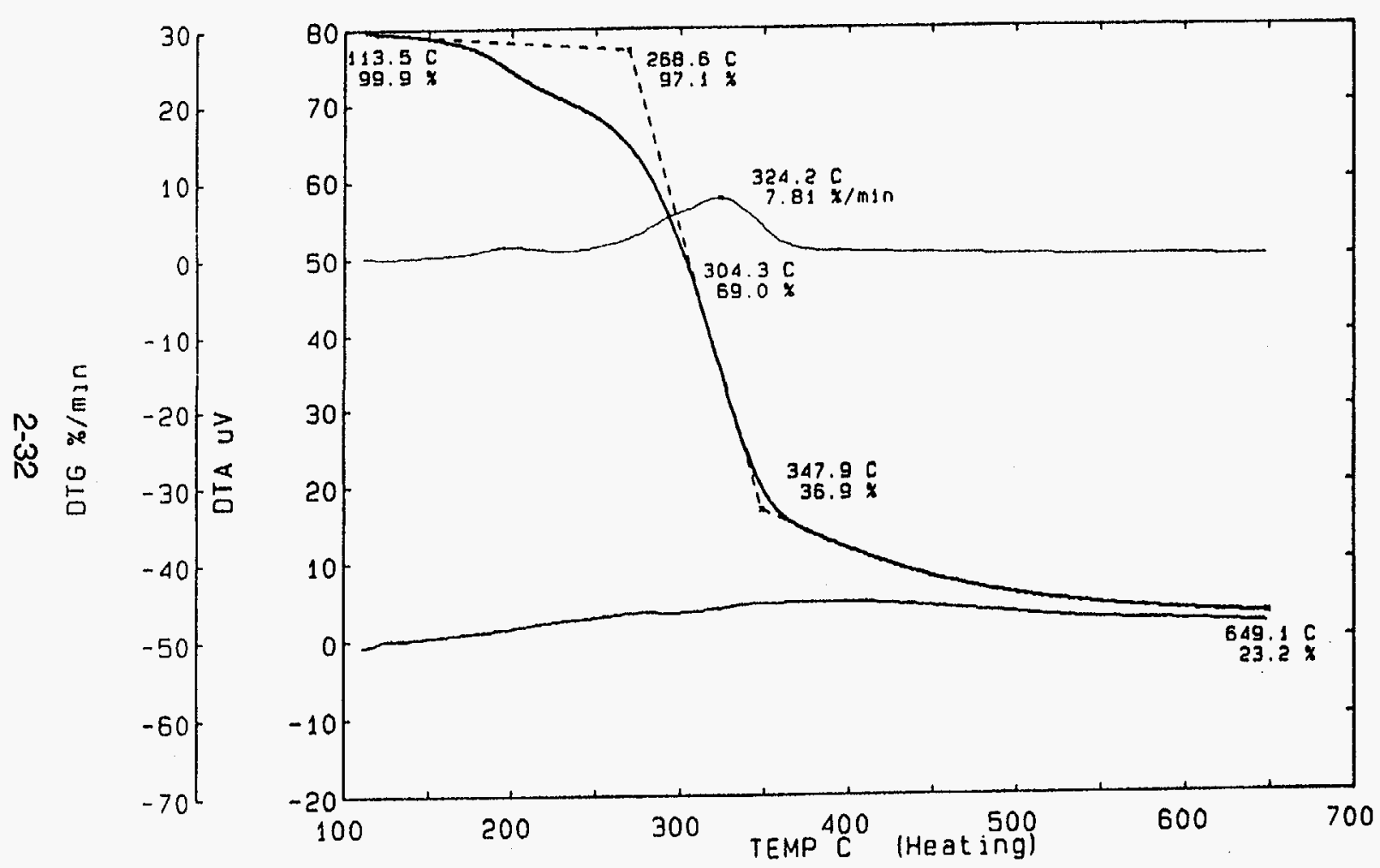

\begin{tabular}{|c|c|}
\hline 90 & $\begin{array}{c}\text { Sorghum } \\
\text { (Sorghum bicolor) }\end{array}$ \\
\hline 80 & Heating rate- $0^{\circ} \mathrm{C} / \min : 10$ \\
\hline \multirow[t]{2}{*}{70} & Sample weight-mg: 3.947 \\
\hline & $\mathrm{T}_{\text {wilut }}{ }^{\circ} \mathrm{C}: 113.5$ \\
\hline 60 & Volatilization \\
\hline \multirow{3}{*}{$\begin{array}{ll}50 \\
40 \\
40\end{array}$} & $T_{\text {oun:pobser }}{ }^{\circ} \mathrm{C}: 268.6$ \\
\hline & $T_{\text {mblpolnx }}{ }^{\circ}{ }^{\circ}: 304.3$ \\
\hline & $T_{\text {and.polent }}{ }^{-C}$ C: 347.9 \\
\hline \multirow[t]{2}{*}{30} & $T_{\max }{ }^{\circ} \mathrm{C}: 324.2$ \\
\hline & Rate $_{\max }-\mathrm{mg} / \mathrm{min}: 7.81$ \\
\hline 20 & WI. $\%_{\text {Aso } c^{*}: 23.2}$ \\
\hline \multirow[t]{2}{*}{10} & $\Delta \mathrm{H}_{\mathrm{vol}}, \mu \mathrm{\mu v}, \mathrm{sec} / \mathrm{mg}: \mathrm{NS}$ \\
\hline & Proximate analysis \\
\hline \multirow[t]{5}{*}{0} & Volatiles $_{\text {Toul }}-w 1 \%: 77.8$ \\
\hline & Volatiles $_{\omega 00} c^{\cdot w t} \%: 76.8$ \\
\hline & 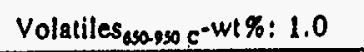 \\
\hline & Fixed carbon-wt\%: 21.6 \\
\hline & Ash-wt \%: 0.6 \\
\hline
\end{tabular}

\section{(Sorghum bicolor) \\ Sorghum}




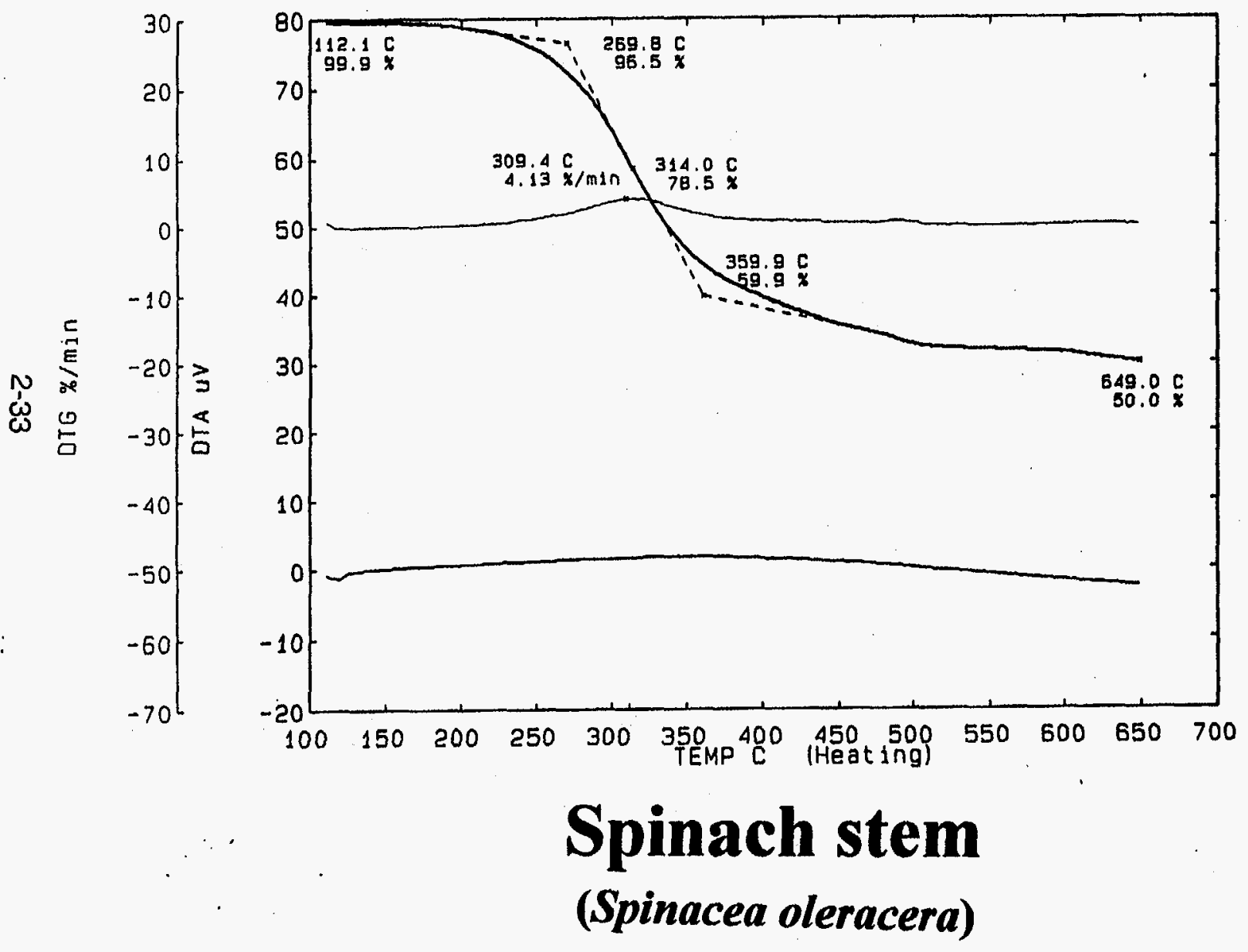

\begin{tabular}{|c|c|}
\hline 90 & $\begin{array}{c}\text { Spinach stem Xylem and } \\
\text { Phloem } \\
\text { (Spinacea oleracea) }\end{array}$ \\
\hline \multirow[t]{2}{*}{80} & \\
\hline & Heating rate- ${ }^{\circ} \mathrm{C} / \mathrm{min}: 10$ \\
\hline 70 & Sample weight-mg: 0.929 \\
\hline \multirow{2}{*}{60} & $\mathrm{~T}_{\text {thllut| }}{ }^{\circ} \mathrm{C}: 112.1$ \\
\hline & Volatilization \\
\hline \multirow{3}{*}{$\begin{array}{ll}50 \\
40\end{array}$} & $T_{\text {ountpoint }}{ }^{\circ} \mathrm{C}: 269.8$ \\
\hline & $T_{\text {mbl-porm }}{ }^{-0} \mathrm{C}: 314.0$ \\
\hline & $T_{\text {end.polm }}-^{\circ} \mathrm{C}: \quad 359.9$ \\
\hline 30 & $\mathrm{~T}_{\mathrm{mm}}{ }^{-{ }^{\circ} \mathrm{C}:} 309.4$ \\
\hline \multirow{2}{*}{20} & Rate $_{\text {max }}-\mathrm{mg} / \mathrm{min}: 4.13$ \\
\hline & Wt. $\%_{\text {asso }} c^{-:}: 50.0$ \\
\hline 10 & Delta $H_{\text {vol. }}$-uv.sec/mg: NS \\
\hline \multirow{2}{*}{0} & Proximate analysis \\
\hline & 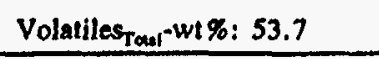 \\
\hline & Volatiles $_{\Delta x a c}-$ wt \%: 50.0 \\
\hline & Volatiles $_{S 60,050} c^{-w t} \%: 3.7$ \\
\hline & Fixed carbon-wt\%: 11.8 \\
\hline & Ash-w1\%: 34.5 \\
\hline
\end{tabular}



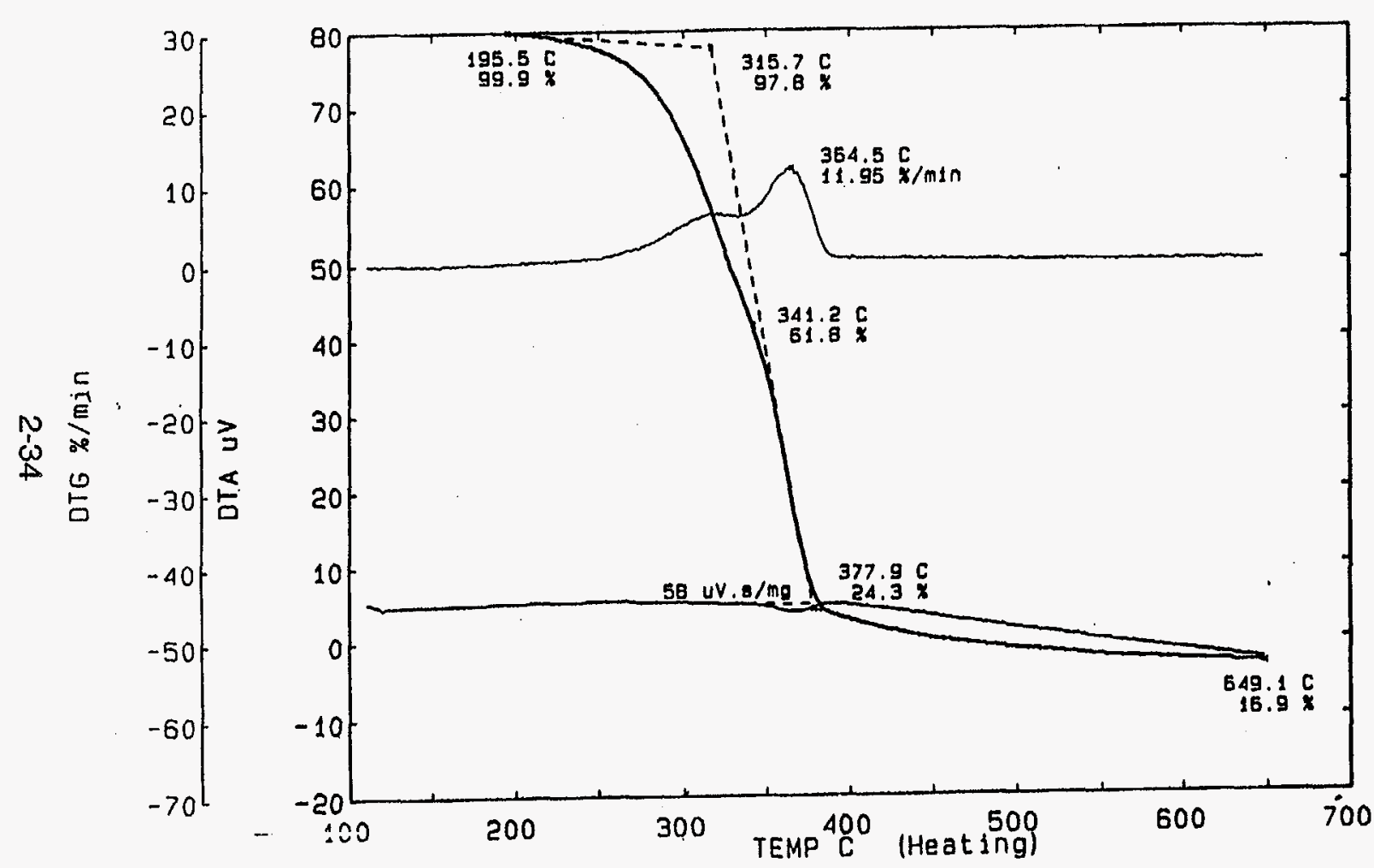

100

90

Bo

70

60

50

$x$

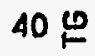

30

20

10

0

\title{
Sugar cane baggase
}

\author{
(Saccharum officinarum)
}

\begin{tabular}{|c|}
\hline $\begin{array}{l}\text { Sugar cane baggase stored for } \\
\text { zero weeks } \\
\text { (Saccharum officinarum) }\end{array}$ \\
\hline Heating rate- ${ }^{\circ} \mathrm{C} / \mathrm{min}: 10$ \\
\hline Sample weight-mg: 2.480 \\
\hline$T_{\text {tollat }}{ }^{\circ} \mathrm{C}: 195.5$ \\
\hline Volatilization \\
\hline$T_{\text {surmplax }}^{-0}{ }^{-0}: 315.7$ \\
\hline$T_{\text {milpolax }}{ }^{\circ} \mathrm{C}: 341.2$ \\
\hline$T_{\text {end.polat }}{ }^{-0} \mathrm{C}: 377.9$ \\
\hline$T_{\operatorname{men}}{ }^{\circ} \mathrm{C}: 364.5$ \\
\hline Rate $_{\max }-\mathrm{mg} / \mathrm{min}: 11.95$ \\
\hline Wt.\% oso c: 16.9 \\
\hline$\Delta \mathrm{H}_{\text {val. }}-\mu \mathrm{v} . \mathrm{sec} / \mathrm{mg}: 58$ \\
\hline Proximate analysis \\
\hline Volatiles $_{\text {Toul }}-w 1 \%: 83.5$ \\
\hline 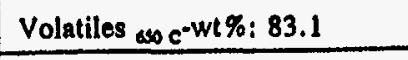 \\
\hline Volatiles asasso $c^{-W 1 \%: 0.4}$ \\
\hline Fixed carbon-wt\%: 14.7 \\
\hline Ash-wt \%: 1.8 \\
\hline
\end{tabular}



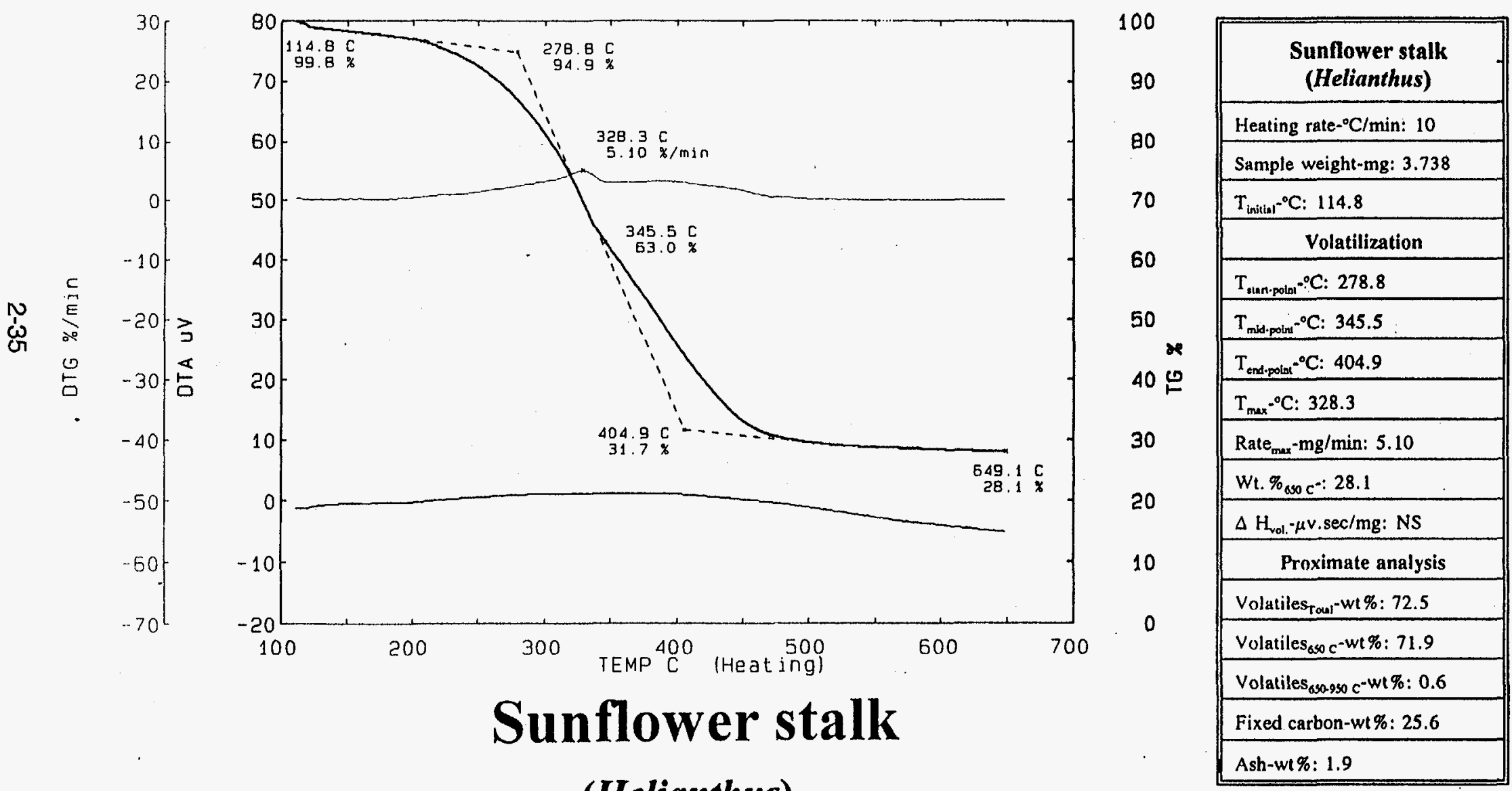

(Helianthus) 

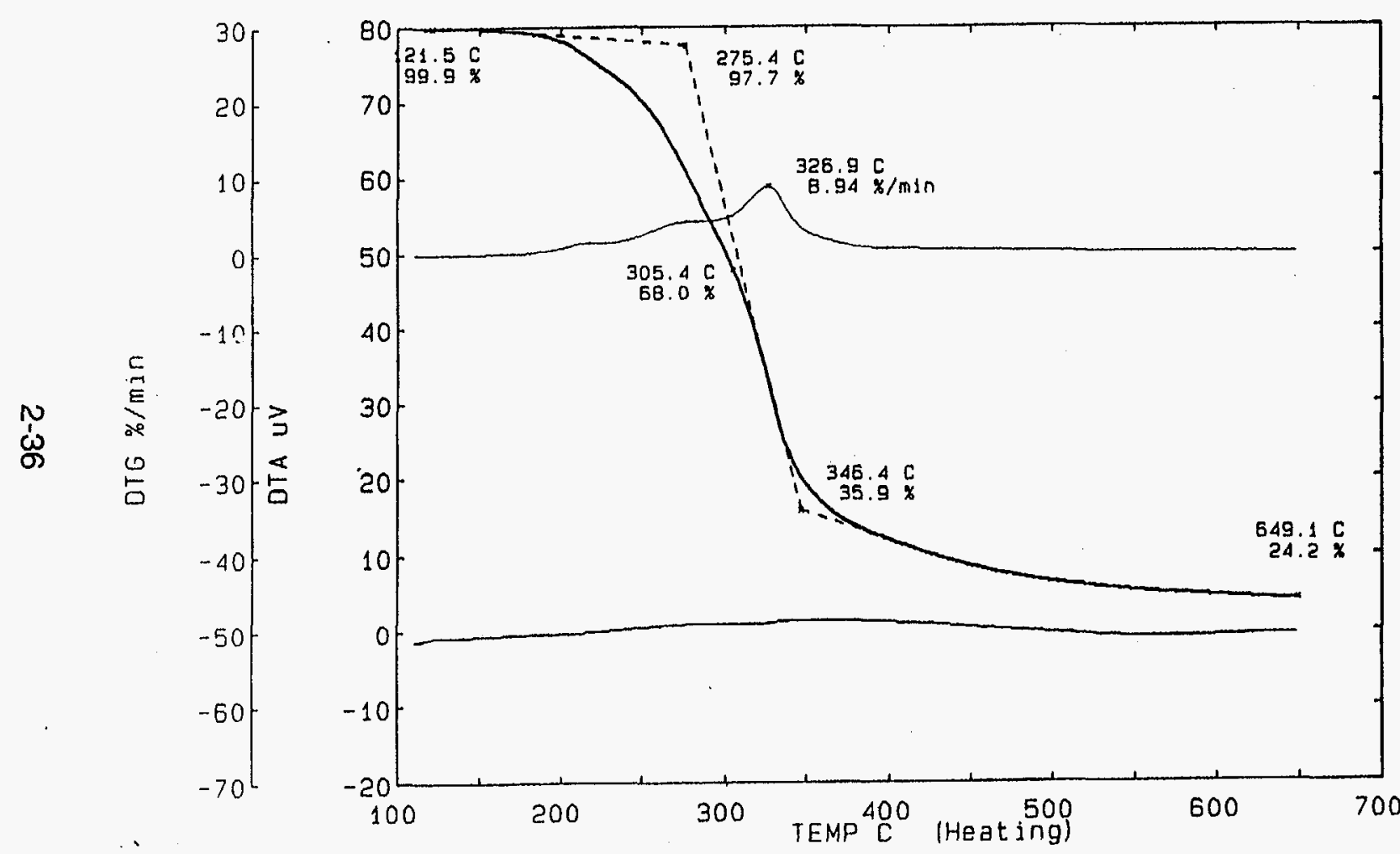

\section{Switch grass}

(Panicum virgatum L.)
100

90

80

70

60

50

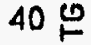

30

20

10

0
Heating rate- ${ }^{\circ} \mathrm{C} / \mathrm{min}: 10$

Sample.weight-mg: 4.445

$T_{\text {tnllula }}{ }^{\circ} \mathrm{C}: 121.5$

Yolatilization

$T_{\text {our:posin }}{ }^{\circ} \mathrm{C}: 275.4$

$\mathrm{T}_{\text {mb-potnt }}{ }^{\circ} \mathrm{C}: 305.4$

$\mathrm{T}_{\text {axt-polst }}{ }^{-\mathrm{C}} \mathrm{C}: 346.4$

$\mathrm{T}_{\max }{ }^{-0} \mathrm{C}: 326.9$

Rate $_{\text {mex }}$-mg/min: 8.94

$W_{t} \% \%_{850} \mathrm{c}^{-:} 24.2$

$\Delta \mathrm{H}_{\mathrm{vol}}-\mu \mathrm{v}, \mathrm{sec} / \mathrm{mg}: \mathrm{NS}$

Proximate analysis

Volatiles $_{\text {Tous }}-\mathrm{wt} \%: 77.0$

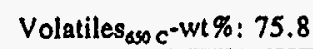

Volatiles $600.950 \mathrm{c}^{-w t} \%: 1.2$

Fixed carbon-wt $\%: 21.2$

Ash-wt\%: 1.8 

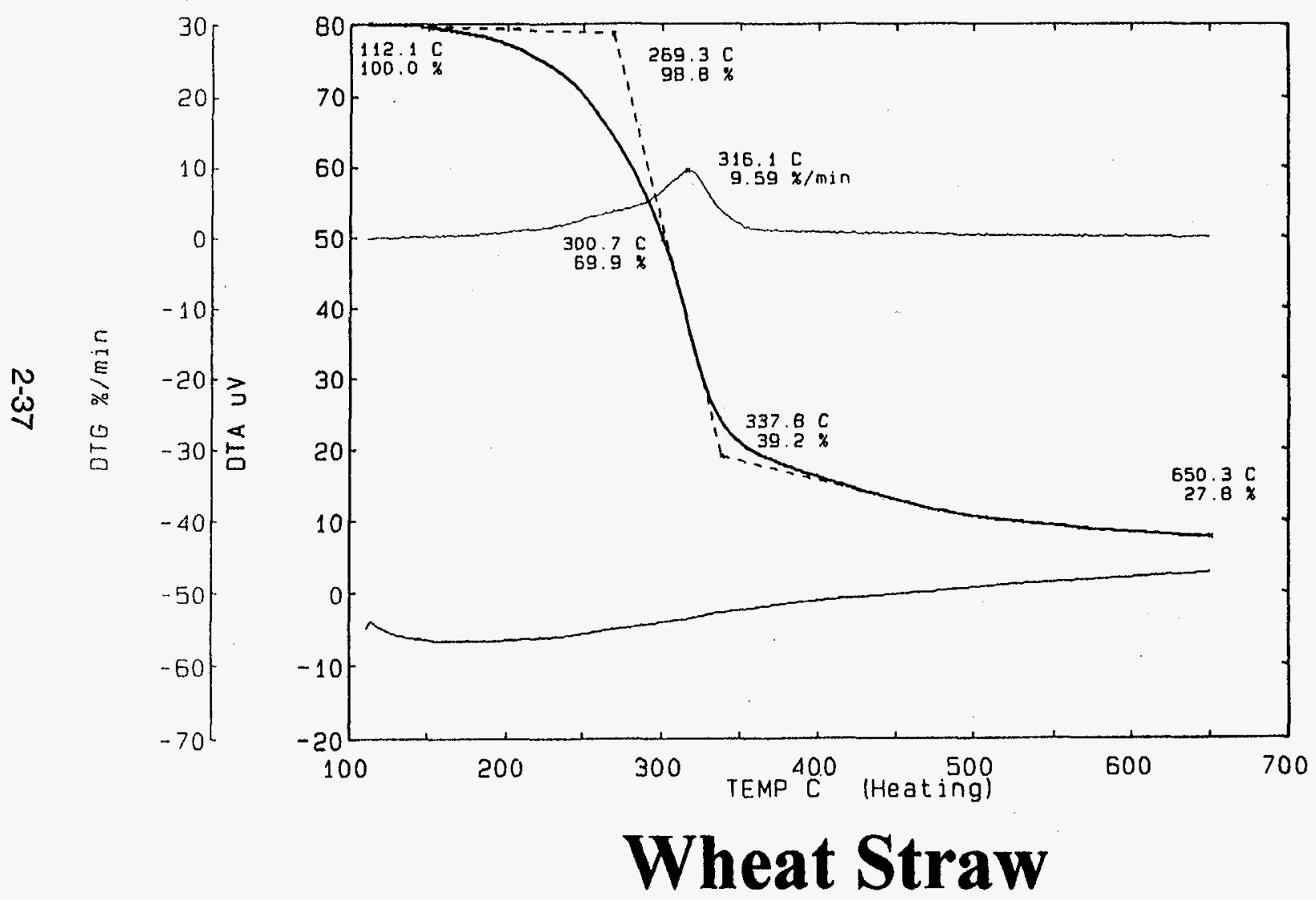

\begin{tabular}{||l||}
100 \\
90
\end{tabular}

(Triticum aestivum, var Thunderbird) 

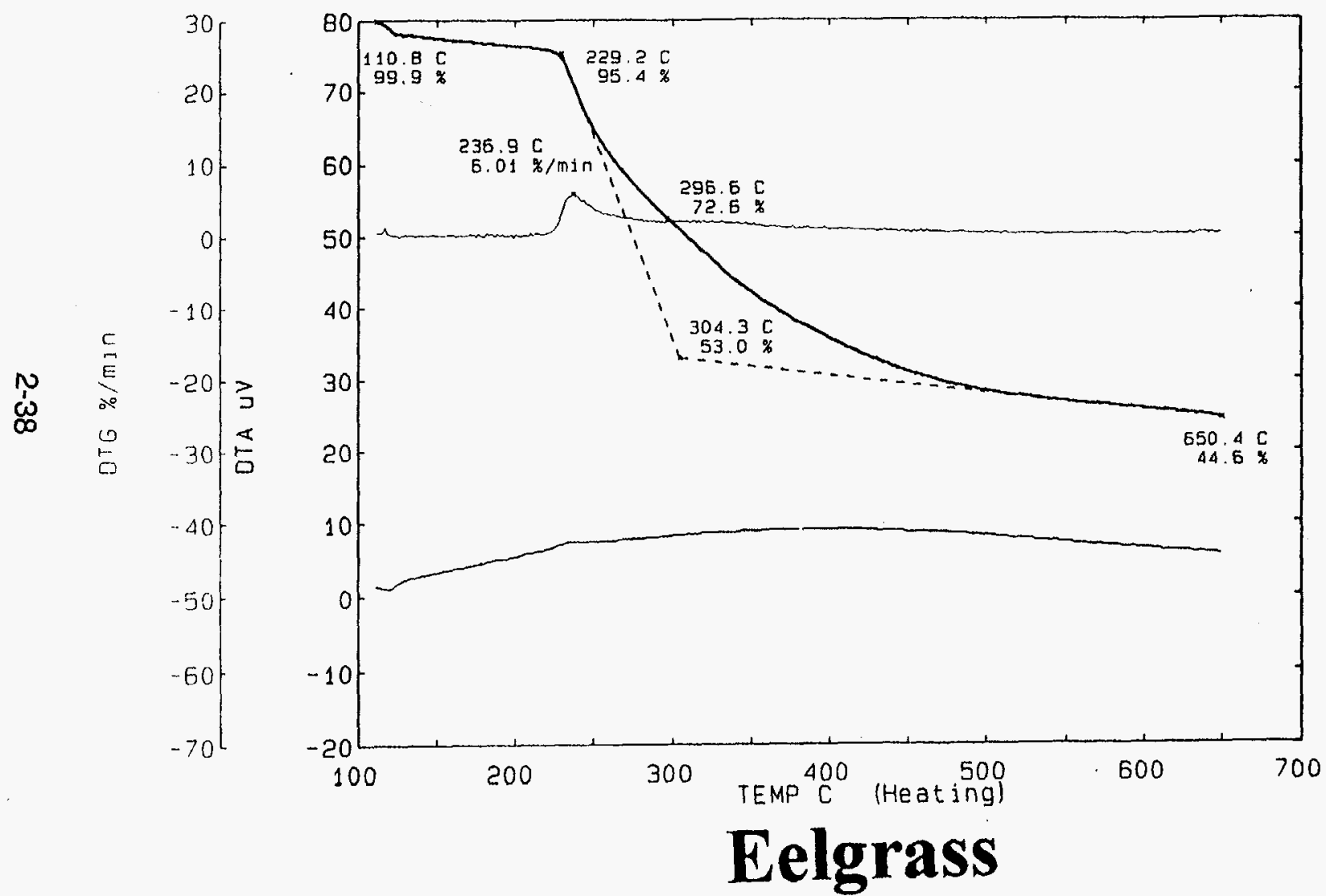

(Zostera marina)

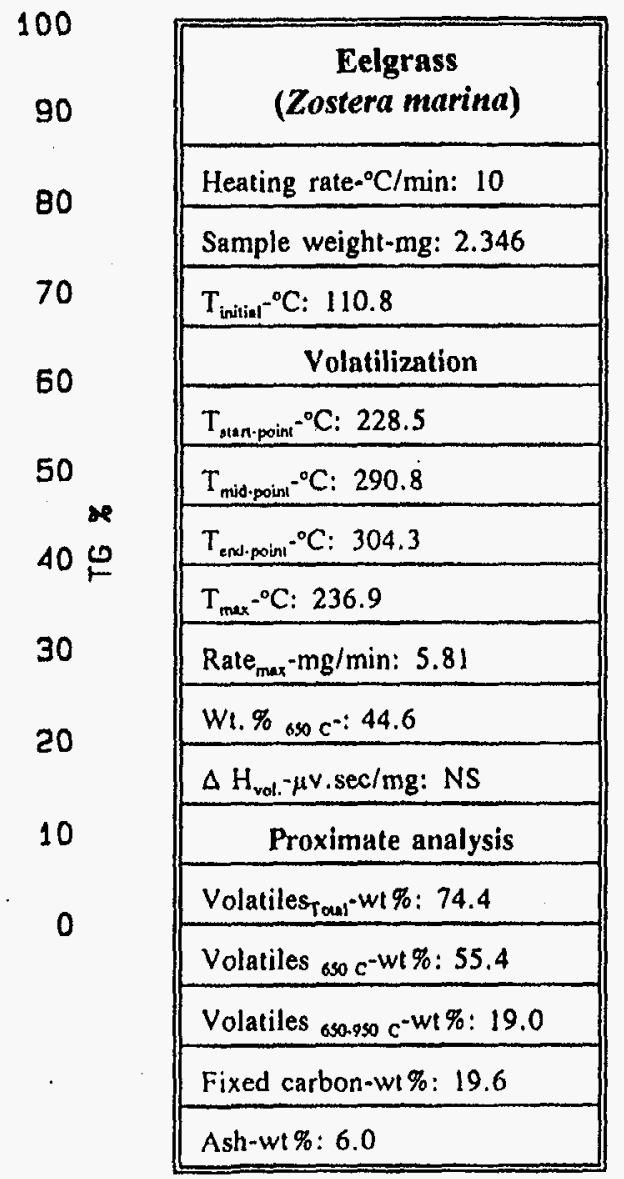



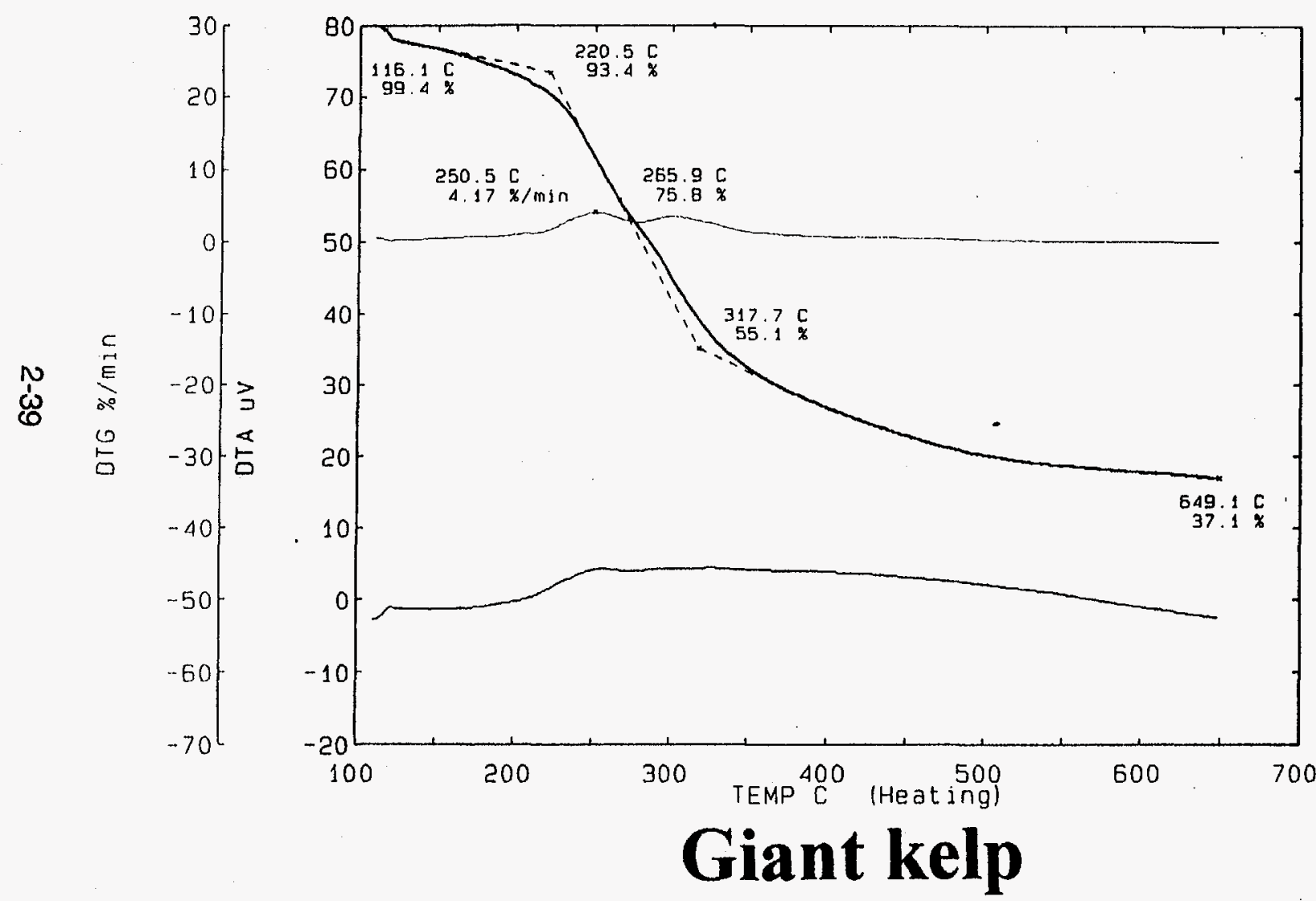

100

80

70

60

50

se

$40 \%$

30

20

10

0

(Laminariaceae)

\begin{tabular}{|c|}
\hline $\begin{array}{c}\text { Giant kelp } \\
\text { (Laminariaceae) }\end{array}$ \\
\hline Heating rate ${ }^{\circ} \mathrm{C} / \mathrm{min}: 10$ \\
\hline Sample weight-mg: 8.672 \\
\hline $\mathrm{T}_{\text {inimilat }}{ }^{\circ} \mathrm{C}: 116.1$ \\
\hline Volatilization \\
\hline $\mathrm{T}_{\text {aun-point }}{ }^{-\mathrm{C}} \mathrm{C}: 220.5$ \\
\hline$T_{\text {mis-poin }}{ }^{-} \mathrm{C}: 265.9$ \\
\hline$T_{\text {end-polin }}{ }^{\circ} \mathrm{C}: \quad 317.7$ \\
\hline$T_{\max }{ }^{\circ} \mathrm{C}: 250.5$ \\
\hline Rate $_{\max }-\mathrm{mg} / \mathrm{min}: 4.17$ \\
\hline Wt.\% aso c-: 37.1 \\
\hline$\Delta \mathrm{H}_{\mathrm{vol} .}-\mu \mathrm{v} . \mathrm{sec} / \mathrm{mg}: \mathrm{NS}$ \\
\hline Proximate analysis \\
\hline Volatiles $_{\text {Tout }}-w 1 \%: 73.9$ \\
\hline Volatiles ${ }_{a s 0} c^{-w 1 \%:} 62.9$ \\
\hline Volatiles sso-9so $c^{-w 1} \%: 11.0$ \\
\hline Fixed carbon-wt \%: 17.9 \\
\hline Ash-wt \%: 8.2 \\
\hline
\end{tabular}



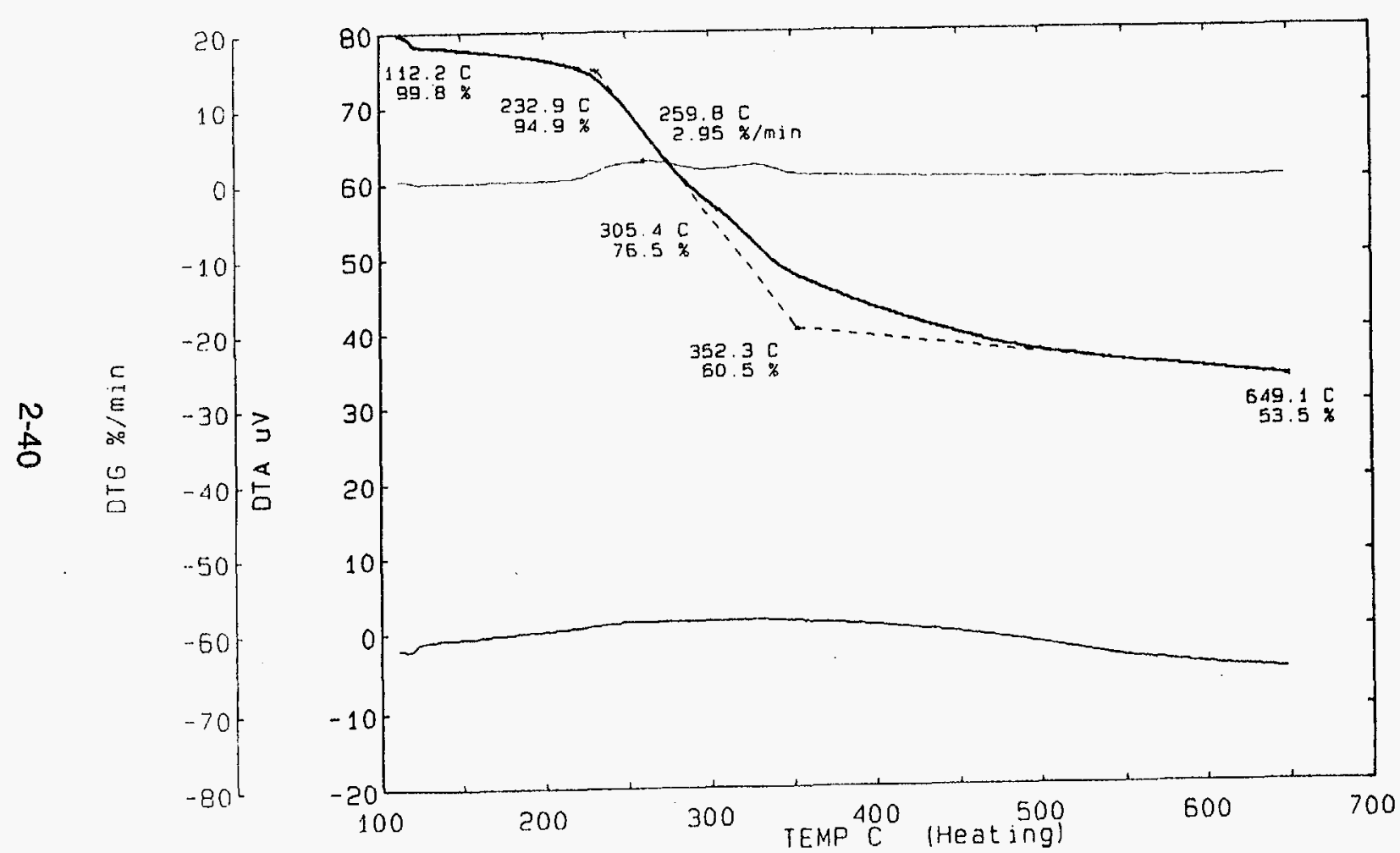

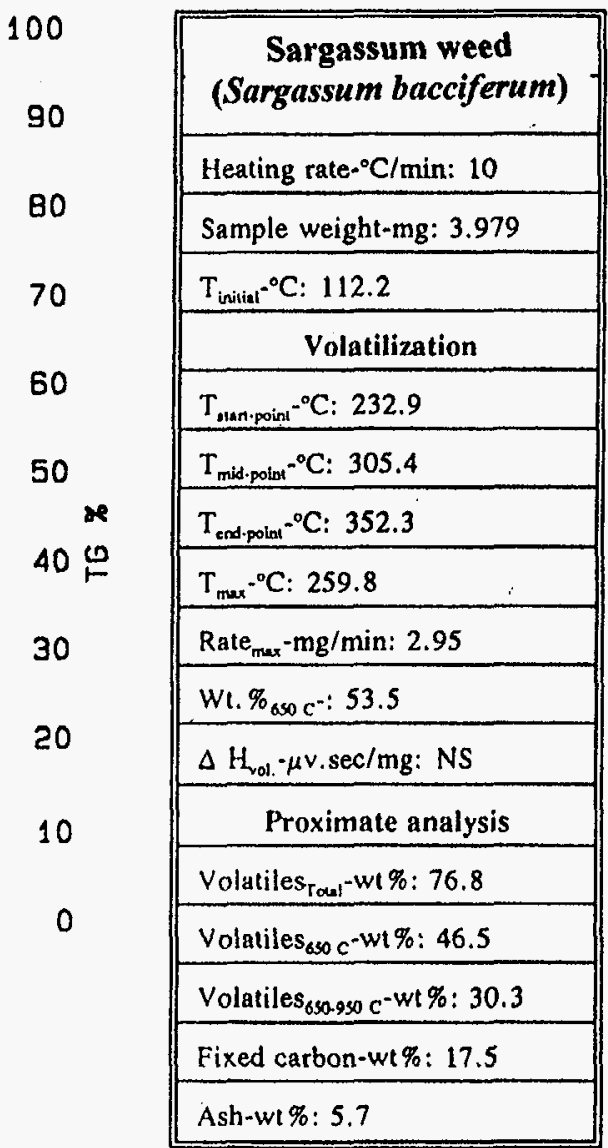

\section{(Sargassum bacciferum)}




\section{Chapter 3}

\section{PROCESSED BIOMASS}

\subsection{Paper}

The manufacture of paper represents the largest use of wood. Paper can also be manufactured from other biomass fibers such as rags or kenaff [Weber, 1982]. In general newsprint is relatively low in ash, while magazine grade paper is high in ash (i.e. $30 \%$ ) due to the clay or other filler used for whiteness. Chemical pulping produces a business paper from softwood called lightweight paper and the one produced from hardwoods is called as heavy paper for example manila folders.

The papers used in this Atlas were obtained from The Institute for Paper Science and Technology, located at Georgia Tech in Atlanta, Ga [IPST, 1993].

\subsection{Densified Biomass}

Biomass occurs in many forms, often of very low density, unsuitable for storage and transportation as fuel. The density of biomass can be increased many times by briquetting, cubing or pelletizing processes in which pressures up to $10^{3}$ atmospheres squeeze out the void space and cement the particles together [Reed, 1978; Bain, 1981]. However, it is observed that the thermal decomposition parameters are very little affected by the processing.

\subsection{Observations}

Since paper is primarily composed of the cellulose fibers of wood, it is not surprising that the thermograms all have midpoints in the $315^{\circ} \mathrm{C}-350^{\circ} \mathrm{C}$ range (like cellulose itself). Possibly a paper chemist would see significant differences. 

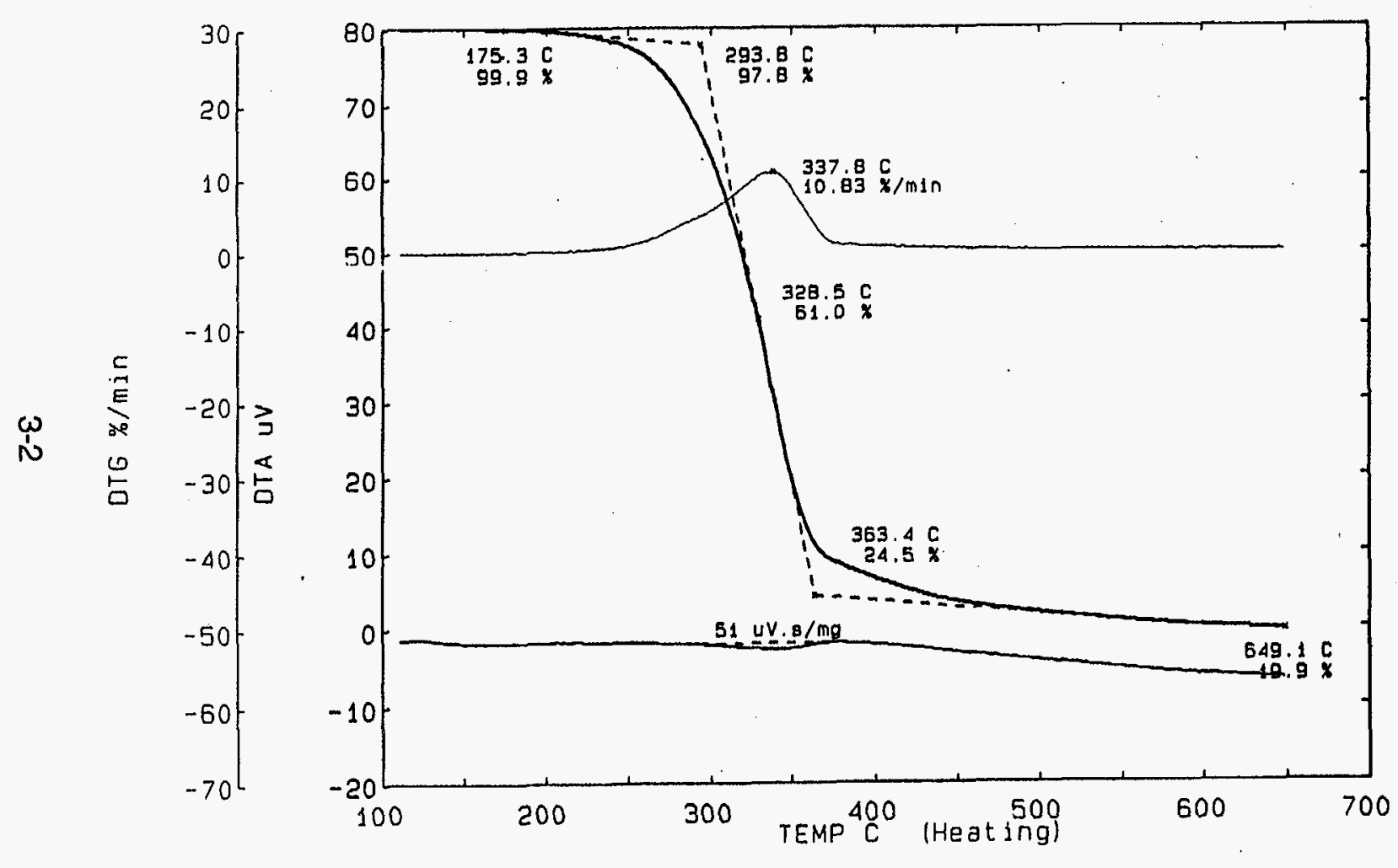

\begin{tabular}{|c|c|}
\hline 90 & Paper, Newsprint \\
\hline \multirow{2}{*}{80} & Heating rate- ${ }^{\circ} \mathrm{C} / \mathrm{min}: 10$ \\
\hline & Sample weight-mg:3.863 \\
\hline 70 & $\mathrm{~T}_{\text {|linit| }}{ }^{-0} \mathrm{C}: 175.3$ \\
\hline \multirow{2}{*}{60} & Volatilization \\
\hline & $\mathrm{T}_{\text {ount-platex }}{ }^{\circ} \mathrm{C}: 293.8$ \\
\hline \multirow{3}{*}{$\begin{array}{r}50 \\
40 \stackrel{0}{4}\end{array}$} & $\mathrm{~T}_{\text {mat. polat }}{ }^{\circ} \mathrm{C}: 328.5$ \\
\hline & $\mathrm{T}_{\text {cospodat }}{ }^{-0} \mathrm{C}: 363.4$ \\
\hline & $T_{\max }{ }^{-0} \mathrm{C}: 337.8$ \\
\hline 30 & Rate $_{\max }-\mathrm{mg} / \mathrm{min}: 10.83$ \\
\hline \multirow{2}{*}{20} & Wt. $\%_{0 s 00} c^{-:} 19.9$ \\
\hline & $\Delta \mathrm{H}_{\mathrm{vol}}-\mu \mathrm{v} . \mathrm{sec} / \mathrm{mg}: 51$ \\
\hline 10 & Proximate analysis \\
\hline \multirow{5}{*}{0} & Volatiles $_{\text {Tow }}-w t \%: 80.8$ \\
\hline & Volatiles $_{s 00} c^{-w t} \%: 80.1$ \\
\hline & Volatiles $_{\text {ssa.9so }} c^{-w t} \%: 0.7$ \\
\hline & Fixed carbon-wt \%: 4.1 \\
\hline & Ash-wt\%: 15.1 \\
\hline
\end{tabular}



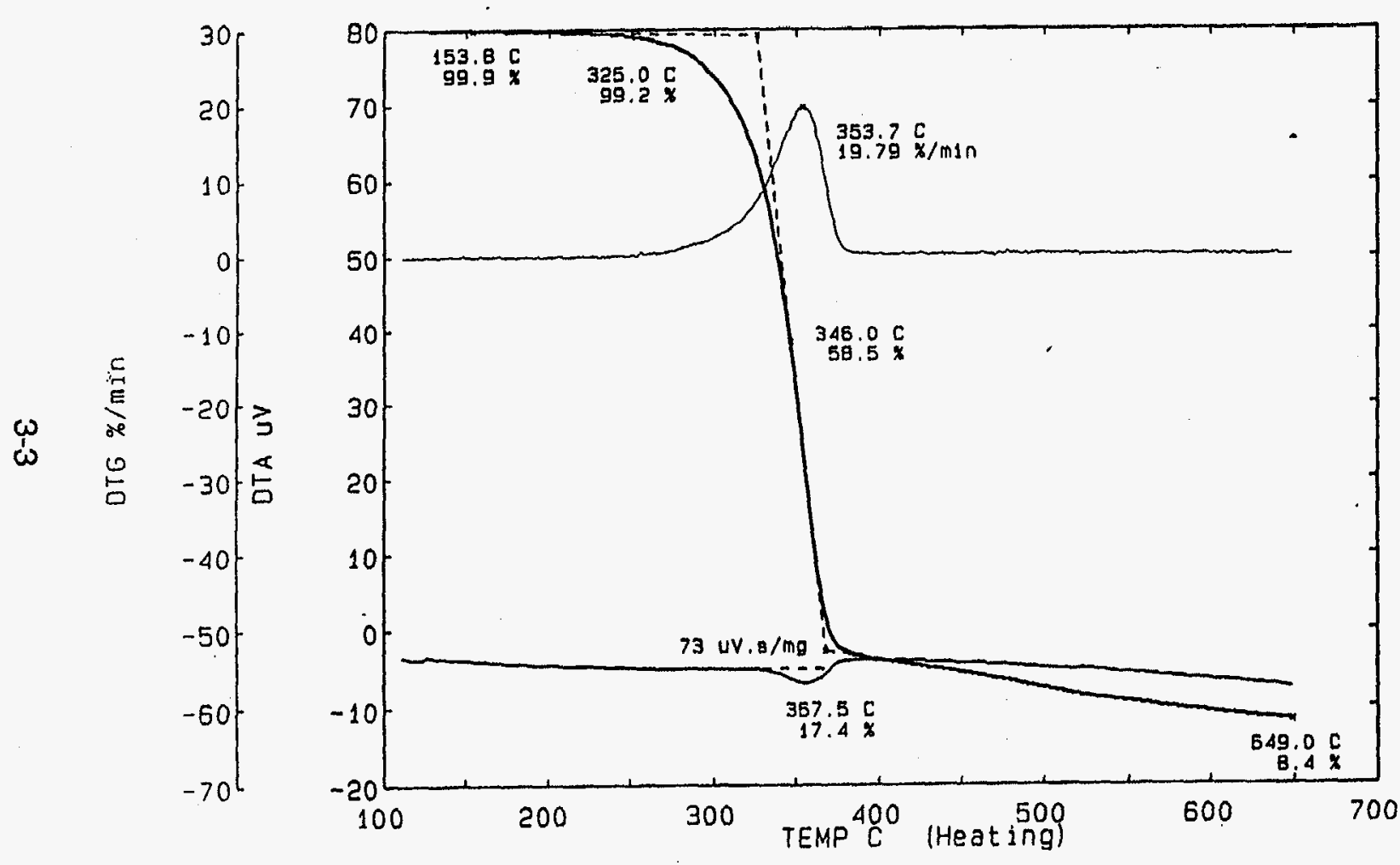

100

90

80

70

60

50

$x$

$40 \stackrel{0}{\leftarrow}$

30

20

10

\section{Paper, Kimwipe tissue}

\begin{tabular}{|c|}
\hline Paper, Kimwipe tissue \\
\hline Heating rate- ${ }^{\circ} \mathrm{C} / \mathrm{min}: 10$ \\
\hline Sample weight-mg: 3.676 \\
\hline$T_{\text {wluat }}{ }^{\circ} \mathrm{C}: 153.8$ \\
\hline Volatilization \\
\hline$T_{\text {oun-polx }} \cdot{ }^{\circ} \mathrm{C}: 325.0$ \\
\hline$T_{\text {mbepoln }}=^{\circ} \mathrm{C}: 346.0$ \\
\hline$T_{\text {exd.polsx }}{ }^{\circ} \mathrm{C}: 367.5$ \\
\hline$T_{\max } 0^{\circ} \mathrm{C}: 353.7$ \\
\hline Rate $_{\max }-\mathrm{mg} / \min : 19.79$ \\
\hline Wt. \% ${ }_{a s o} c^{*:} 8.4$ \\
\hline$\Delta \mathrm{H}_{\mathrm{vol}}, \mu \mathrm{v}, \mathrm{sec} / \mathrm{mg}: 73$ \\
\hline Proximate analysis \\
\hline Volatiles $_{\text {Tout }}-w 1 \%: 95.5$ \\
\hline Volatiles ${ }_{\text {sso }} c^{-w 1} \%: 91.6$ \\
\hline Volatiles ${ }_{650.950} c^{-w t} \%: 3.9^{\circ}$ \\
\hline Fixed carbon-wt $\%: 3.7$ \\
\hline Ash-wt\%: 0.8 \\
\hline
\end{tabular}



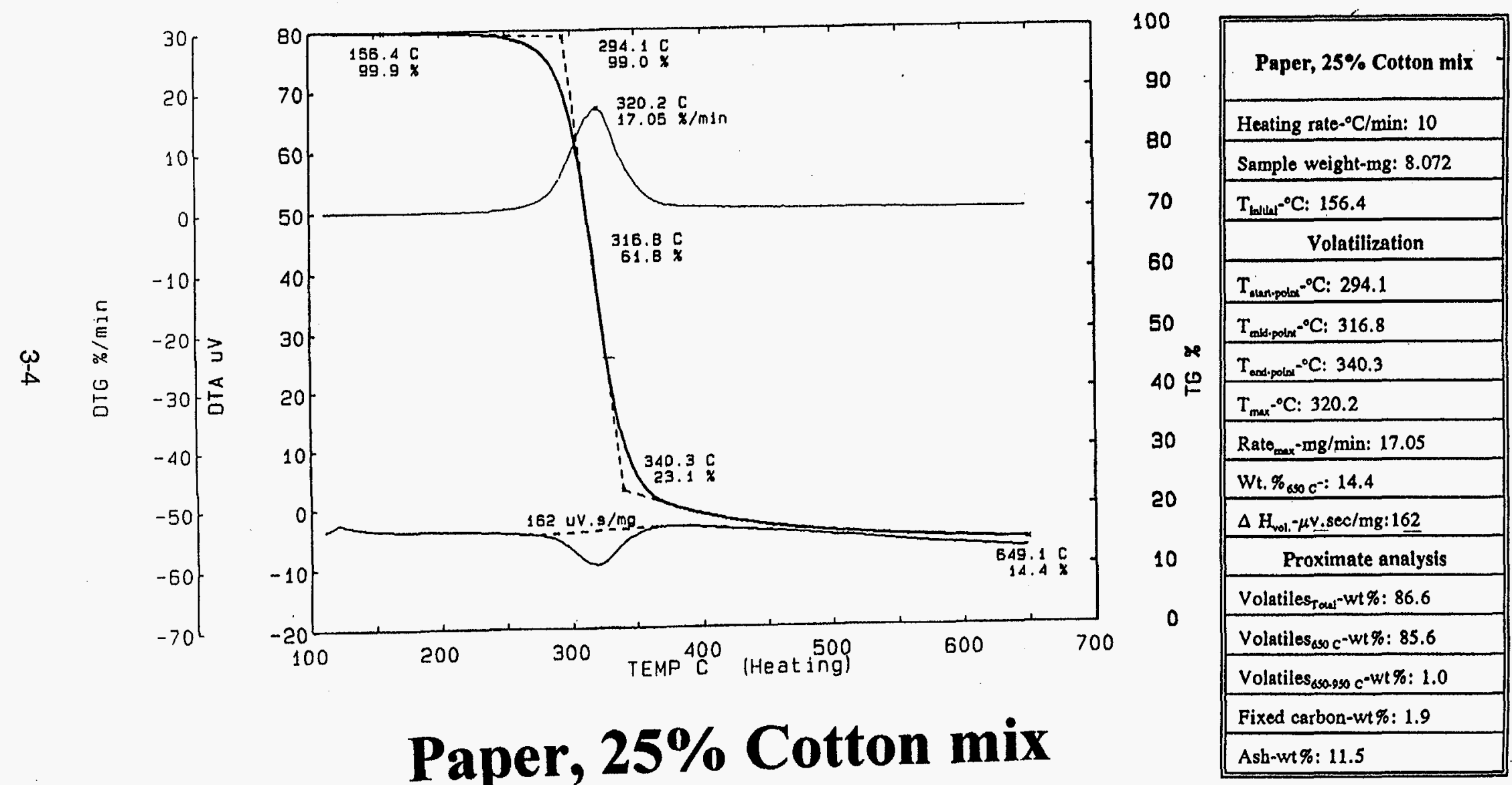

\section{Paper, 25\% Cotton mix}



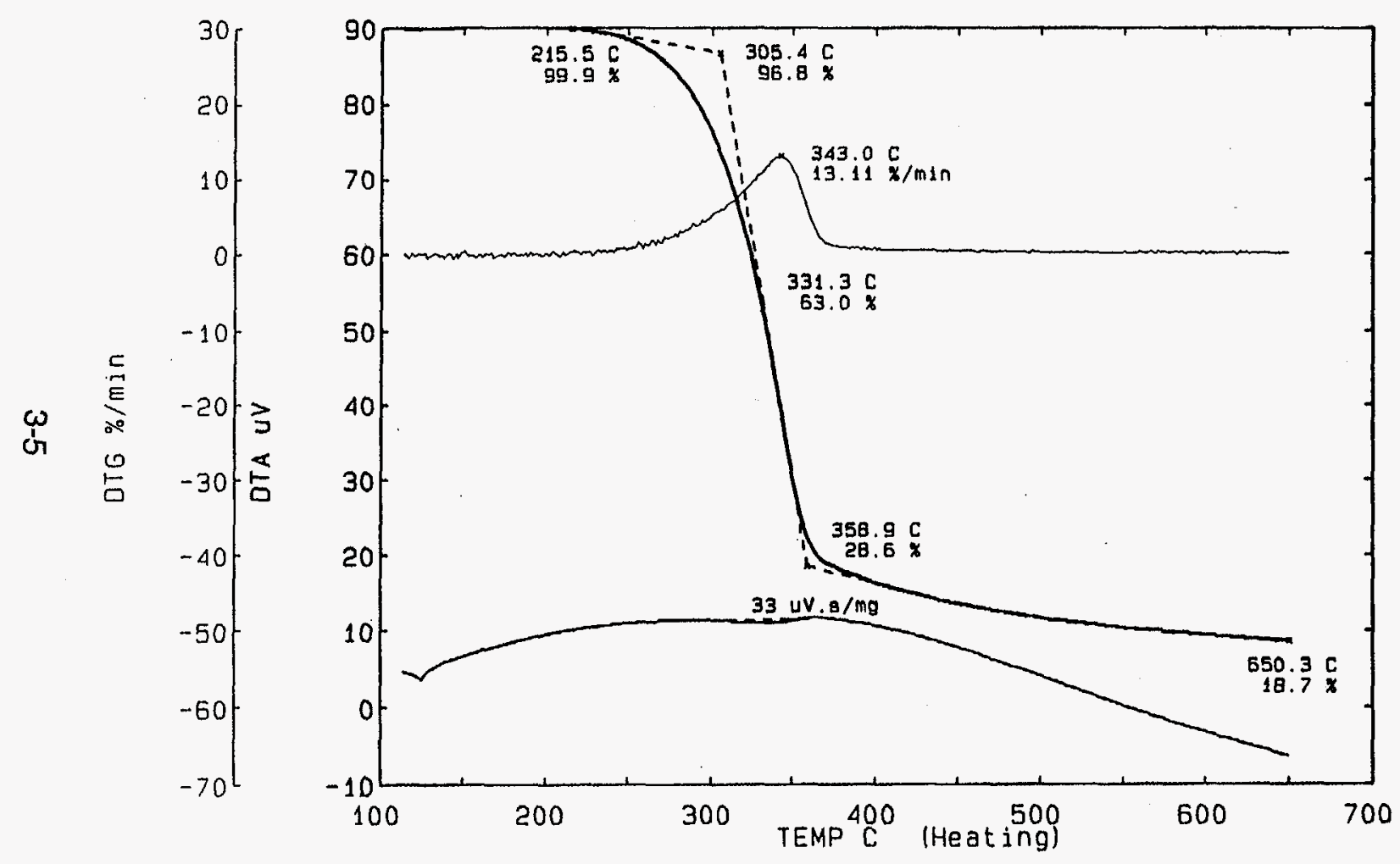

\begin{tabular}{|c|c|}
\hline 90 & Cardboard, corrugated sheet \\
\hline \multirow{2}{*}{80} & Heating rate- ${ }^{\circ} \mathrm{C} / \mathrm{min}: 10$ \\
\hline & Sample weight-mg: 2.128 \\
\hline 70 & 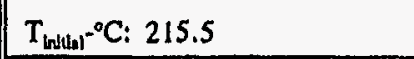 \\
\hline \multirow{2}{*}{60} & Volatilization \\
\hline & $T_{\text {uann-poimm }}{ }^{\circ} \mathrm{C}: 305.4$ \\
\hline 50 & $T_{\text {mid.polx }}{ }^{-0} \mathrm{C}: \quad 331.3$ \\
\hline \multirow{2}{*}{ 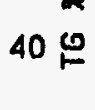 } & $\mathrm{T}_{\text {end-potyx }}{ }^{-0} \mathrm{C}: 358.9$ \\
\hline & $\mathrm{T}_{\operatorname{mex}}{ }^{-} \mathrm{C}: 343.0$ \\
\hline 30 & Rate $_{\max }-\mathrm{mg} / \mathrm{min}: 13.11$. \\
\hline \multirow{2}{*}{20} & Wt. $\%_{\text {Sso }} c^{-:}: 18.7$ \\
\hline & Delta $\mathrm{H}_{\text {vol }}$-uv.sec/mg: 33 \\
\hline 10 & Proximate analysis \\
\hline \multirow{5}{*}{0} & Volatiles $_{\text {Totel }}-\mathrm{w}$ \%: 85.5 \\
\hline & Volatiles $_{\text {cso } c^{-w} w: 81.3}$ \\
\hline & Volatiles $_{650.950} c^{-w t \%: 4.2}$ \\
\hline & Fixed carbon-wt $\%: 13.8$ \\
\hline & Ash-wt\%: 0.7 \\
\hline
\end{tabular}

\section{Cardboard, corrugated sheet}



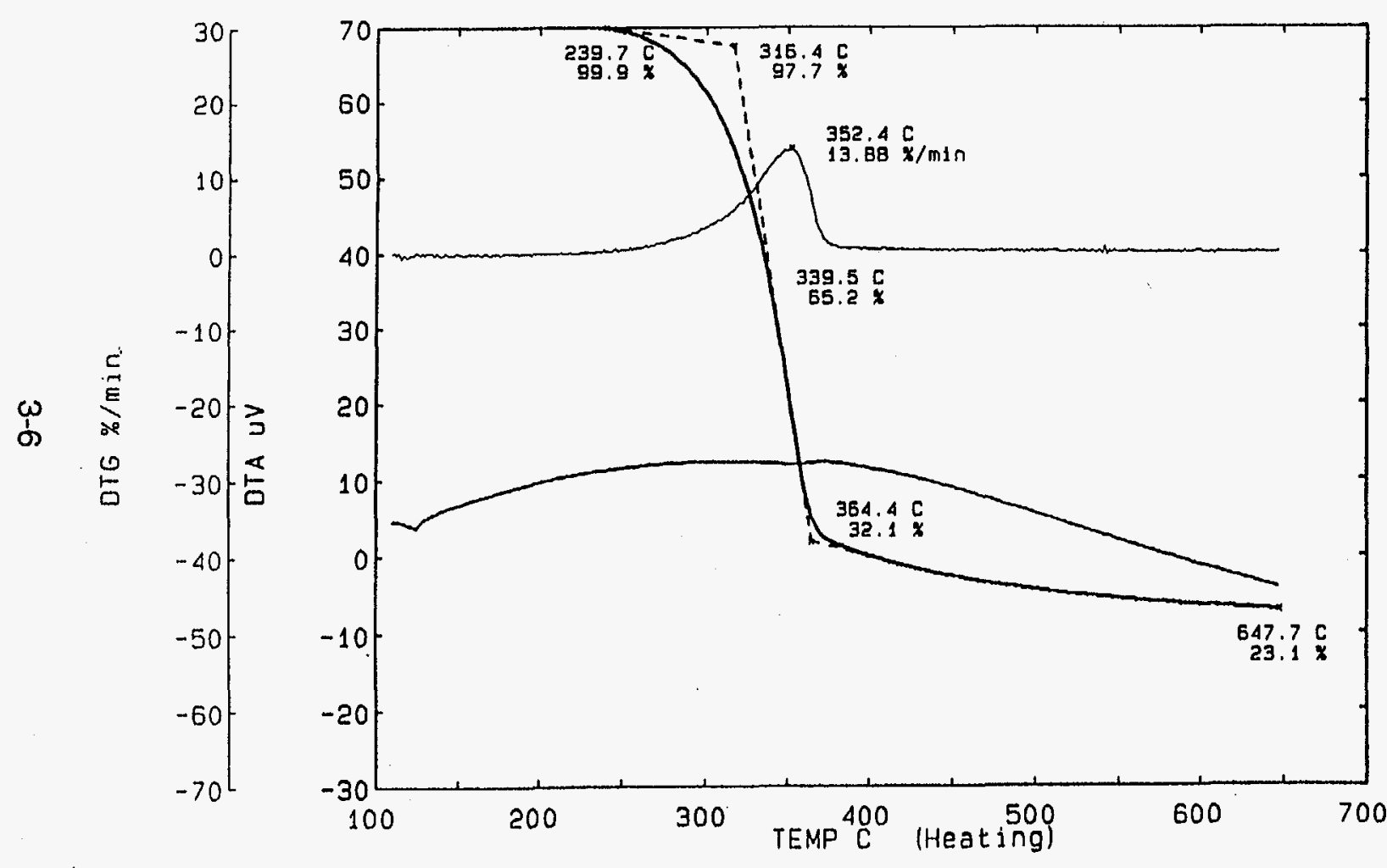

\begin{tabular}{|c|c|}
\hline \multicolumn{2}{|l|}{100} \\
\hline 90 & Cardboard - Liner board \\
\hline \multirow{2}{*}{ BO } & Heating rate- ${ }^{\circ} \mathrm{C} / \mathrm{min}: 10$ \\
\hline & Sample weight-mg: 2.065 \\
\hline 70 & $\mathrm{~T}_{\text {lanilut }}{ }^{-} \mathrm{C}: 239.7$ \\
\hline \multirow{2}{*}{60} & Volatilization \\
\hline & $T_{\text {dann podxt }}{ }^{-} \mathrm{C}: 316.4$ \\
\hline \multirow{3}{*}{$\begin{array}{l}50 \\
40 \stackrel{0}{0} \\
40\end{array}$} & $T_{\text {midpotax }}{ }^{-0} \mathrm{C}: 339.5$ \\
\hline & $T_{\text {eod polat: }}{ }^{\circ} \mathrm{C}: 364.4$ \\
\hline & $T_{\max } x^{\circ} \mathrm{C}: 352.4$ \\
\hline 30 & Rate $_{\max }-\mathrm{mg} / \min : 13.88$ \\
\hline \multirow[t]{2}{*}{20} & Wt. \% ${ }_{630} c^{-:}: 23.1$ \\
\hline & $\Delta \mathrm{H}_{\text {vol. }}-\mu v . \sec / \mathrm{mg}: \mathrm{NS}$ \\
\hline 10 & Proximate analysis \\
\hline \multirow[t]{5}{*}{0} & Volatiles $_{\text {Toul }}-w t \%: 81.2$ \\
\hline & Volatiles ${ }_{6 s 0} \mathrm{c}^{-w t} \%: 76.9$ \\
\hline & Volatiles $_{650-950} \mathrm{c}^{-w 1} \%: 4.3$ \\
\hline & Fixed carbon-wi\%: 12.9 \\
\hline & Ash-wt \%: 5.9 \\
\hline
\end{tabular}




\section{Chapter 4}

\section{MUNICIPAL SOLID WASTE}

\subsection{Municipal Solid Waste}

Municipal solid waste, (MSW), is a potentially large source of several quads of energy as shown in Table 2.1. While it contains some plastics, the major combustible portion is lignocellulosic, and so MSW is classified with biomass. The degradation of MSW is being considered from the view point of environmental aspects as well as a source of energy.

Samples of many of the typical components in MSW are found in the previous chapters. Samples of several well characterized RDFs were obtained from NREL and their thermal data are listed in the Atlas.

\subsection{Plastics}

A significant fraction of RDF is plastics and we show thermograms of the common plastics also obtained from NREL. The midpoint of the breakdown curve is higher than cellulose for all of the plastics shown except a part of PVC and PVA. This makes it easy to distinguish a large component of plastic from biomass in thermograms.

\subsection{Observations}

All three of the MSW samples show a toe of about $10 \%$ on the curve that is probably due to the plastic content. Again, it is possible that thermograms could be used to estimate the plastic content of the samples, since all the samples except PVC decompose at a temperature well above that of cellulose, the marker for paper. 

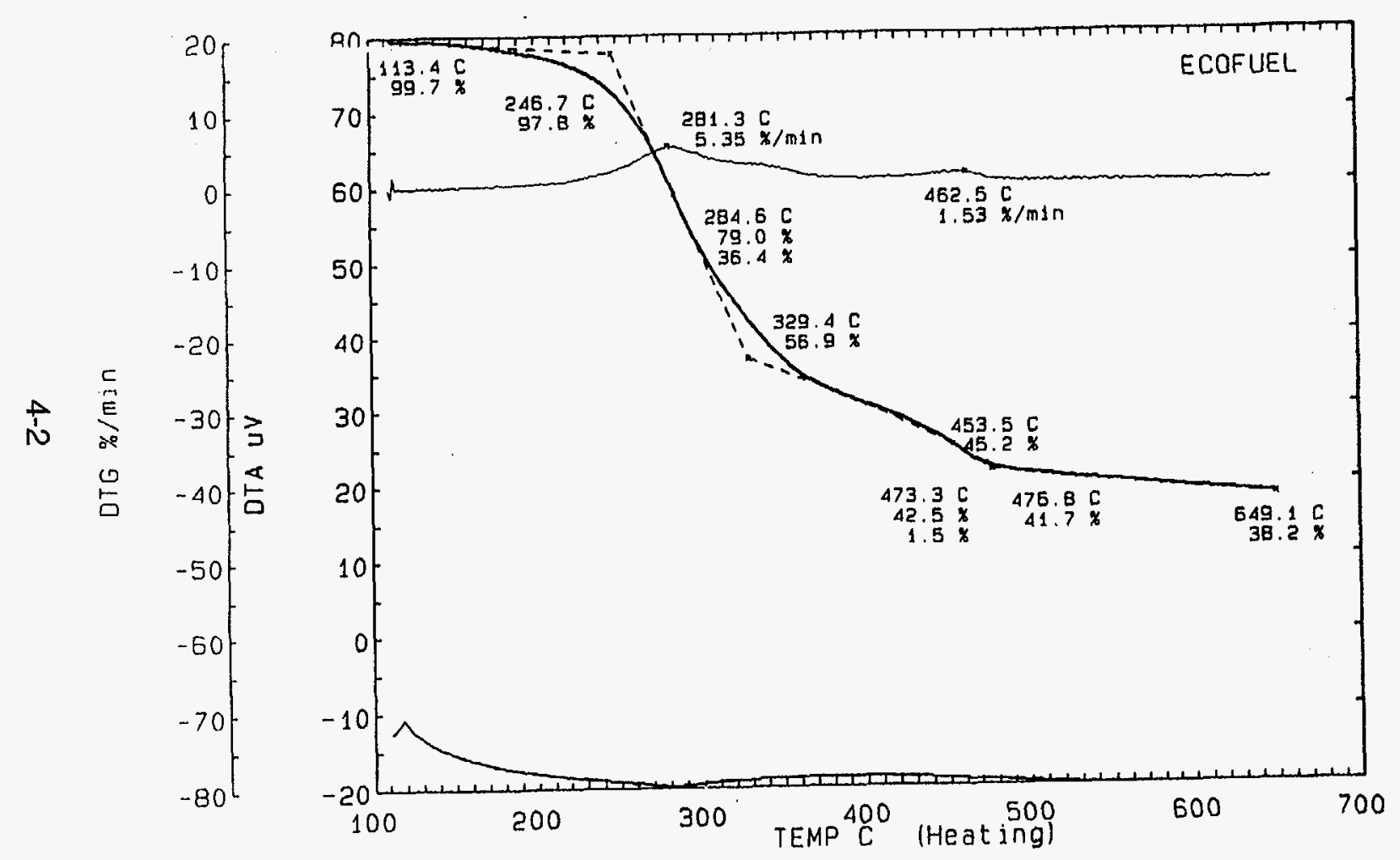

\begin{tabular}{|c|c|}
\hline 90 & $\begin{array}{l}\text { Ecofuel-2 } \\
\text { (Arthur D. Little) }\end{array}$ \\
\hline \multirow[t]{2}{*}{90} & Heating rate- ${ }^{\circ} \mathrm{C} / \mathrm{min}: 10$ \\
\hline & Sample weight-mg: 8.934 \\
\hline 70 & $T_{\text {iniliat }}{ }^{-0} \mathrm{C}: 113.4$ \\
\hline \multirow[t]{2}{*}{60} & Volatilization \\
\hline & 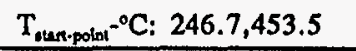 \\
\hline 50 & $\mathrm{~T}_{\text {mul-polex }}{ }^{\circ} \mathrm{C}: 284.6,473.3$ \\
\hline \multirow[t]{2}{*}{ 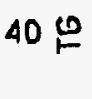 } & $T_{\text {end-pedin }}{ }^{-0} \mathrm{C}: \quad 329.4,476.8$ \\
\hline & $\mathrm{T}_{\max }{ }^{-} \mathrm{C}: 281.3,462.5$ \\
\hline 30 & Rate $_{\max }-\mathrm{mg} / \min : 5.35,1.53$ \\
\hline \multirow[t]{2}{*}{20} & 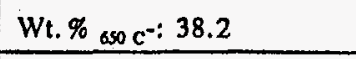 \\
\hline & $\Delta \mathrm{H}_{\mathrm{rol}-1}-\mu \mathrm{v} . \mathrm{sec} / \mathrm{mg}: \mathrm{NS}$ \\
\hline 10 & Proximate analysis \\
\hline \multirow[t]{5}{*}{0} & Volatiles $_{\text {Tout }}-\mathrm{wt} \%: 68.9$ \\
\hline & Volatiles $_{\text {sso }} c^{-w t} \%: 61.8$ \\
\hline & 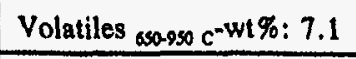 \\
\hline & Fixed carbon-wt\%: 22.6 \\
\hline & Ash-wt \%: 8.5 \\
\hline
\end{tabular}

\section{Ecofuel-2}

(Arthur D. Little) 

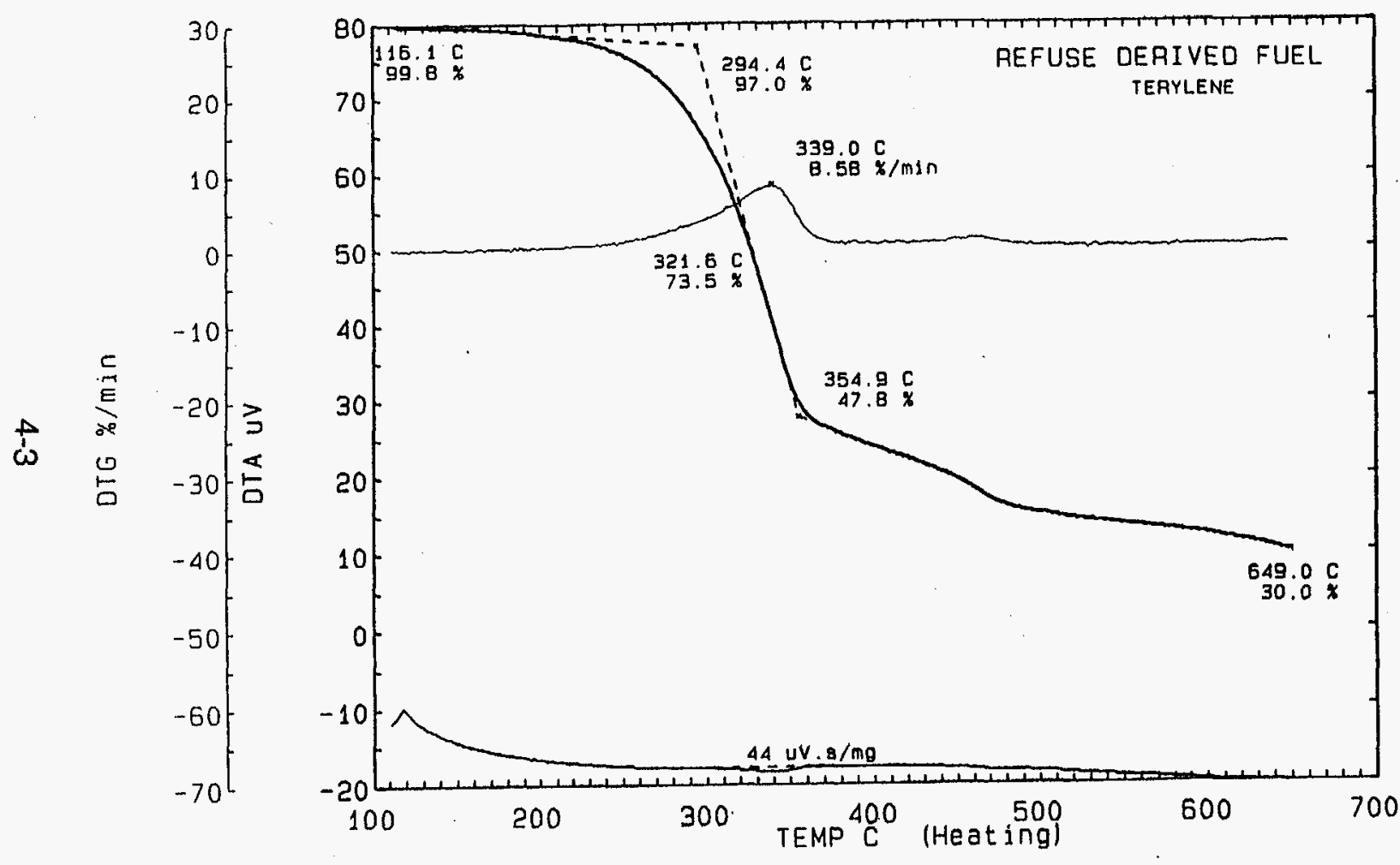

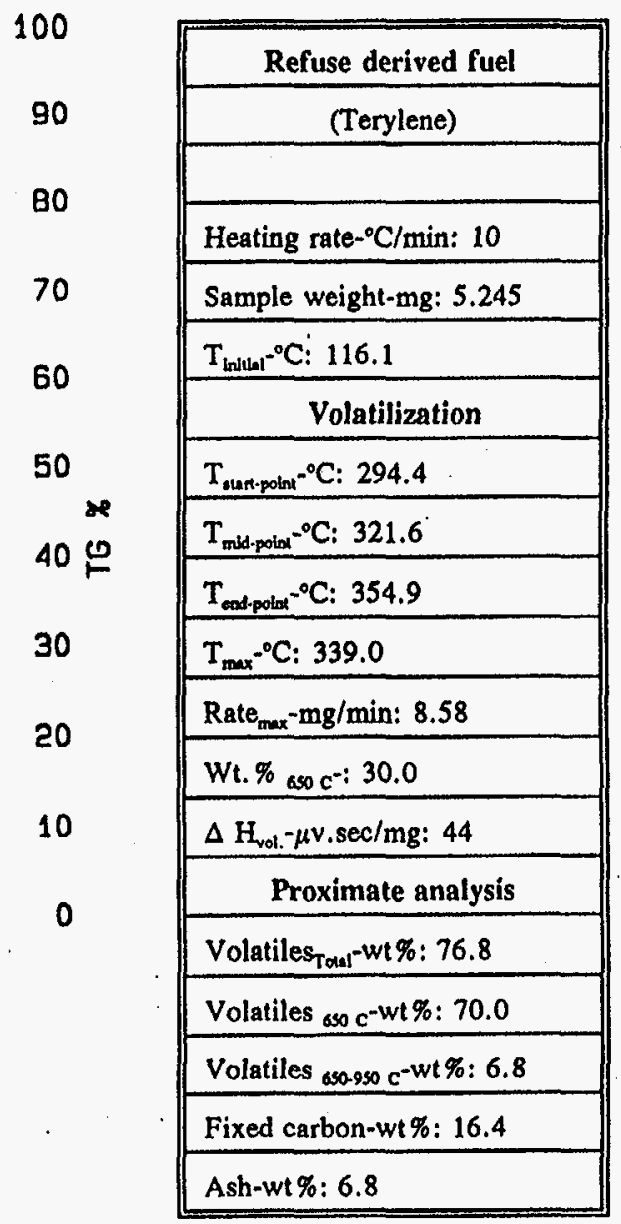

\section{Refuse derived fuel} (Teledyne) 

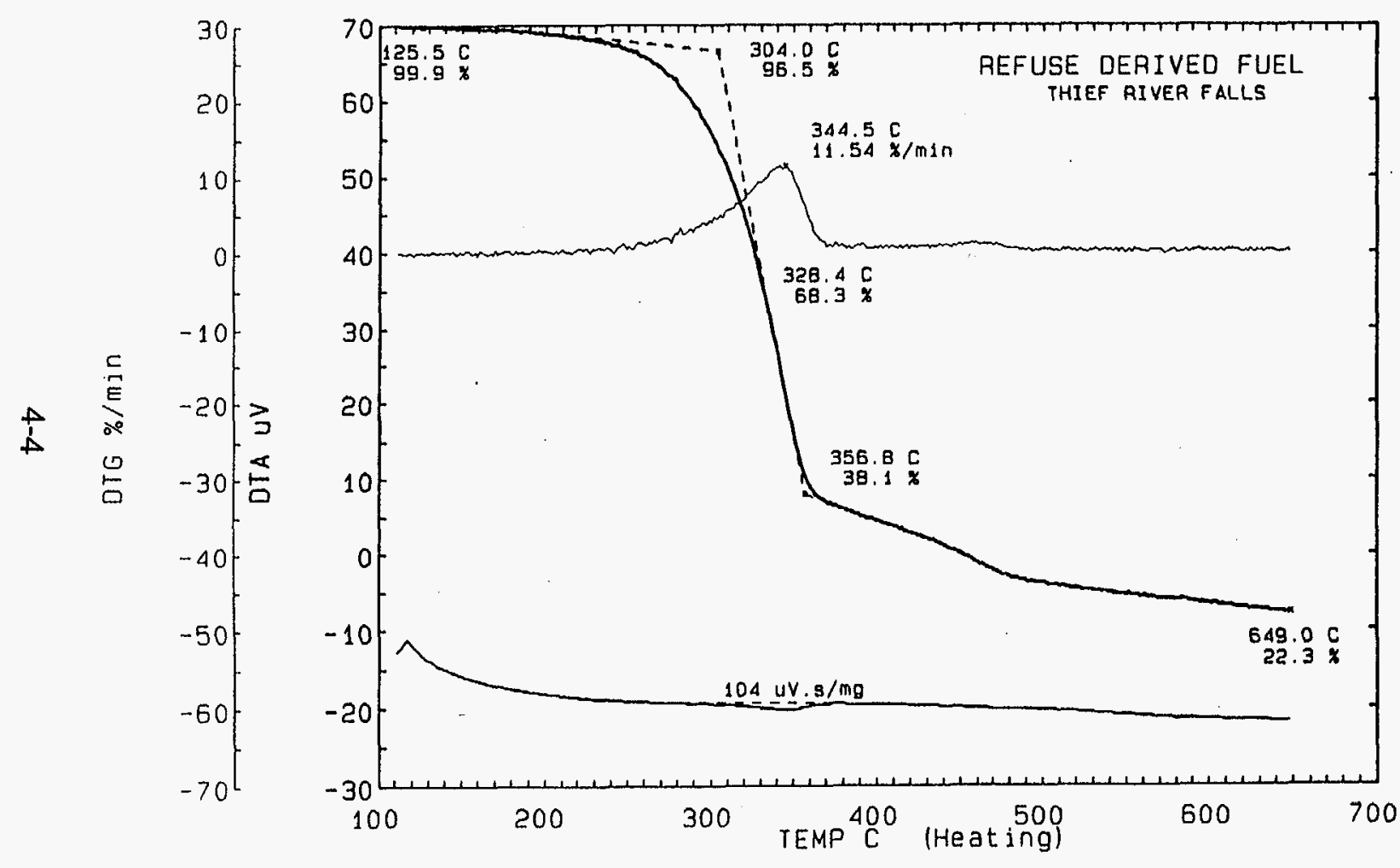

\begin{tabular}{|c|c|}
\hline \multirow[b]{2}{*}{90} & Refuse derived fuel \\
\hline & (Thief river falls) \\
\hline \multirow{2}{*}{80} & \\
\hline & Heating rate $-0^{\circ} \mathrm{C} / \mathrm{min}: 10$ \\
\hline 70 & Sample weight-mg: 4.567 \\
\hline \multirow{2}{*}{60} & $\mathrm{~T}_{\text {miluti: }}{ }^{\circ} \mathrm{C}: 125.5$ \\
\hline & Volatilization \\
\hline \multirow{3}{*}{$\begin{array}{l}50 \\
40 \stackrel{P}{口}\end{array}$} & $T_{\text {mann-polx }}{ }^{-0} \mathrm{C}: 304.0$ \\
\hline & $T_{\text {mid-polx }}{ }^{\circ} \mathrm{C}: 328.4$ \\
\hline & $T_{\text {ent.polnt }}{ }^{-0} \mathrm{C}: 356.8$ \\
\hline 30 & $\mathrm{~T}_{\max }{ }^{\circ} \mathrm{C}: 344.5$ \\
\hline \multirow{2}{*}{20} & Rate $_{\max }-\mathrm{mg} / \mathrm{min}: 11.54$ \\
\hline & Wt. $\%_{a s 0} c^{-:}: 22.3$ \\
\hline \multirow[t]{2}{*}{10} & $\Delta \mathrm{H}_{\mathrm{vol}}-\mu \mathrm{v}, \mathrm{sec} / \mathrm{mg}: 104$ \\
\hline & Proximate analysis \\
\hline \multirow{5}{*}{0} & Volatiles $_{\text {Toul }}-w 1 \%: 80.9$ \\
\hline & Volatiles ${ }_{6 s s} c^{-w t} \%: 77.7$ \\
\hline & Volatiles ${ }_{\text {ssa.9so }} c^{-w t} \%: 3.2$ \\
\hline & Fixed carbon-wt $\%: 84.7$ \\
\hline & Ash-wt\%: 3.8 \\
\hline
\end{tabular}




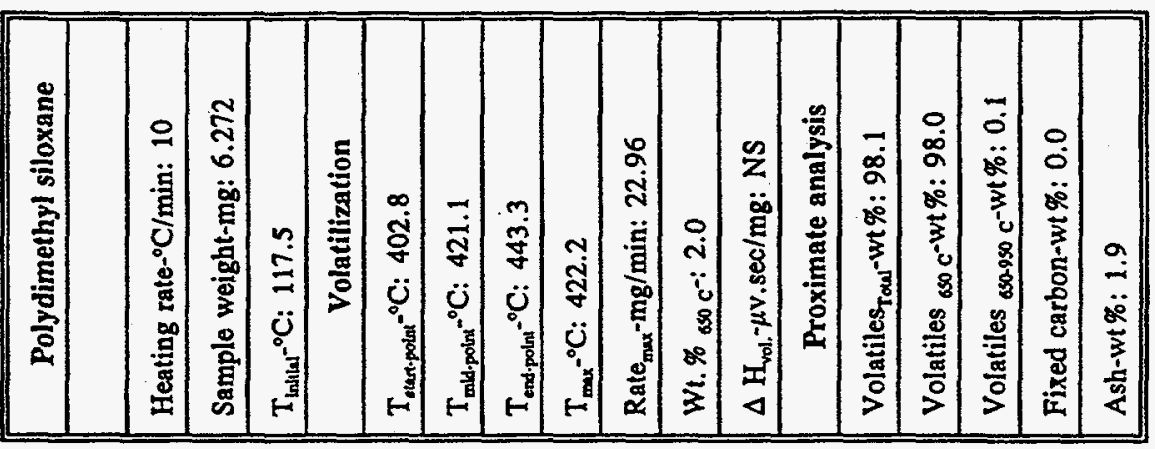

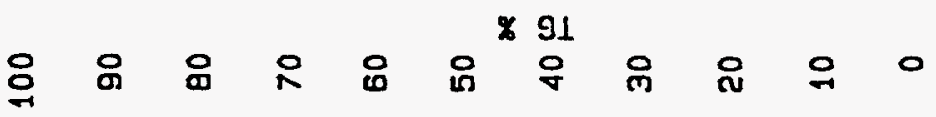

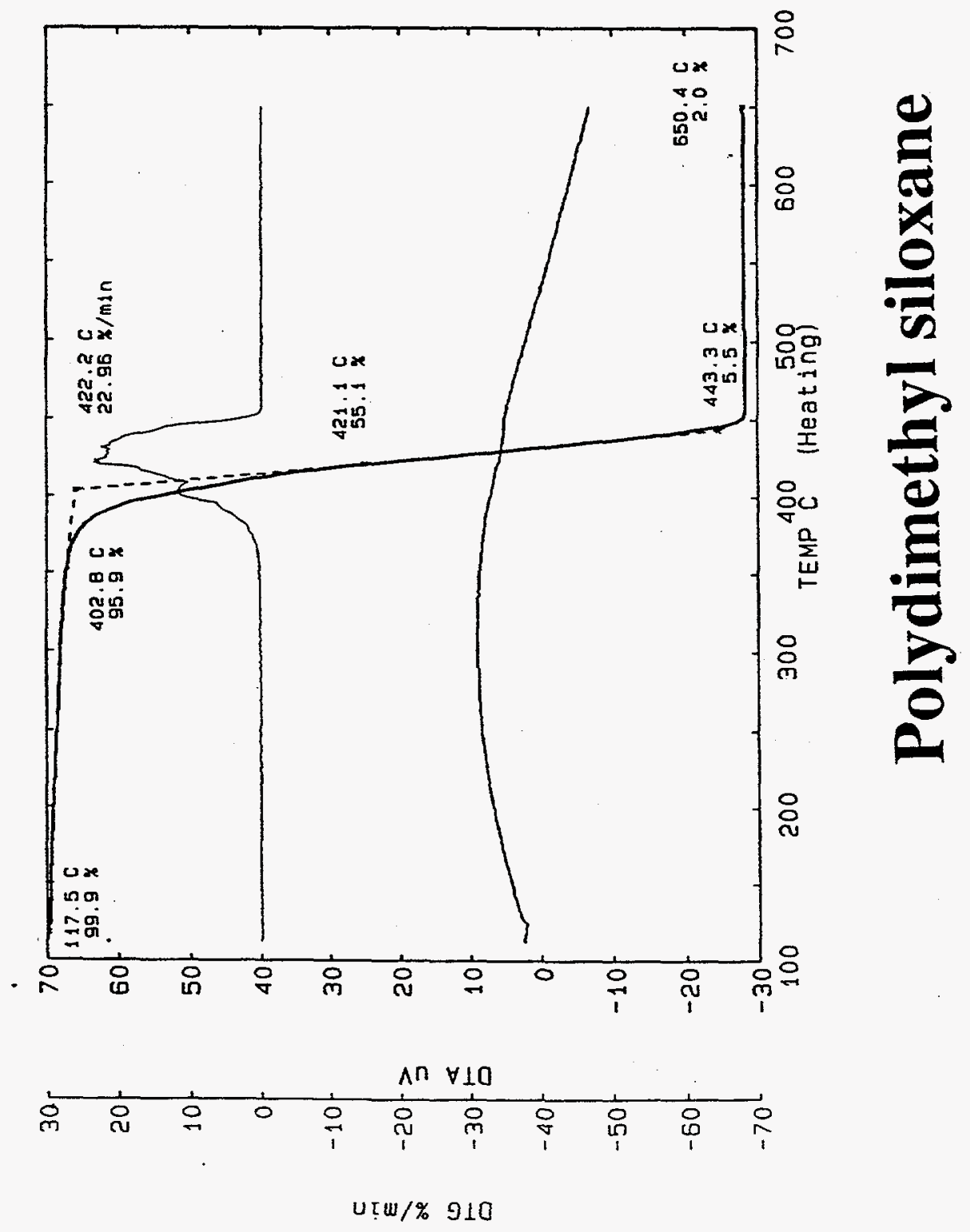




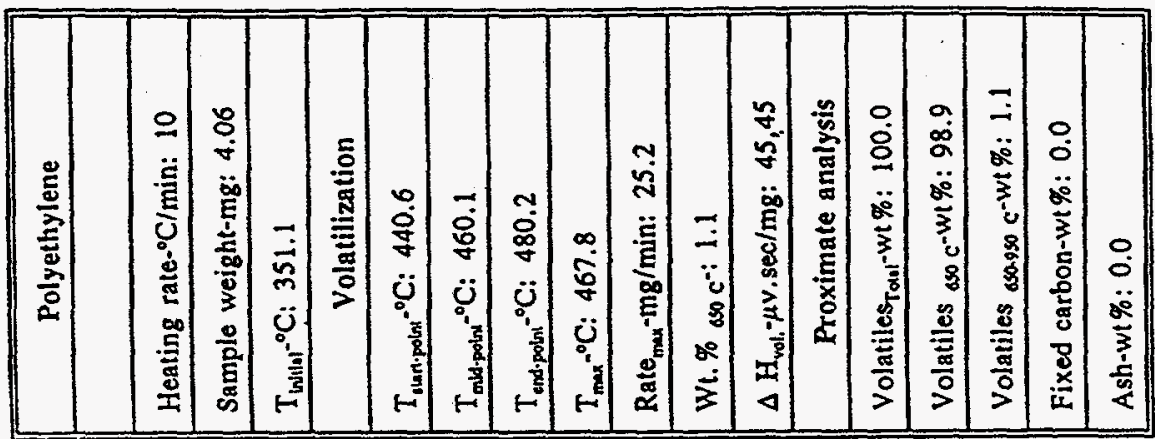

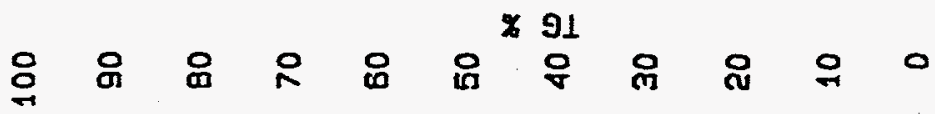

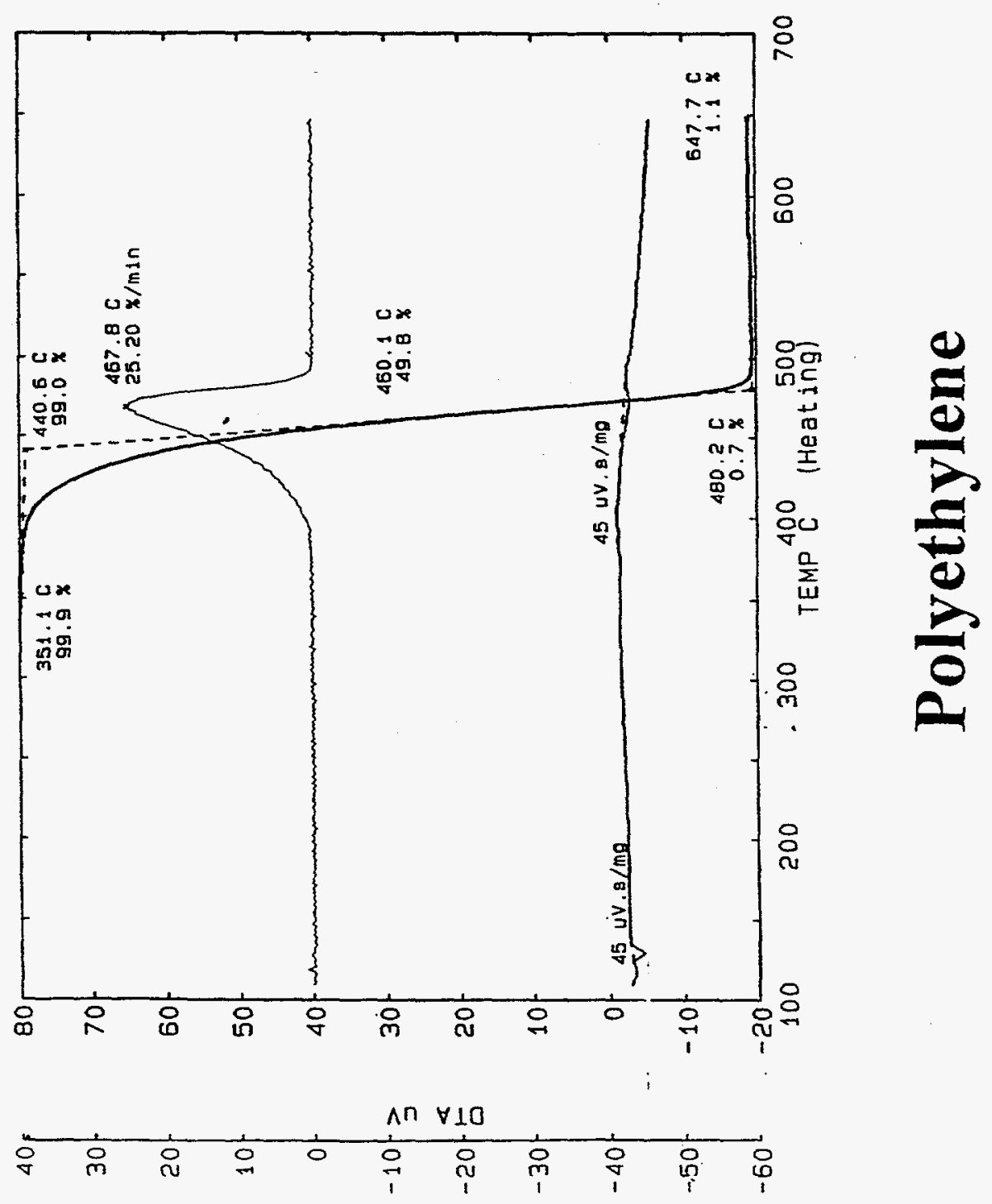

บ โบแ/\% 910 

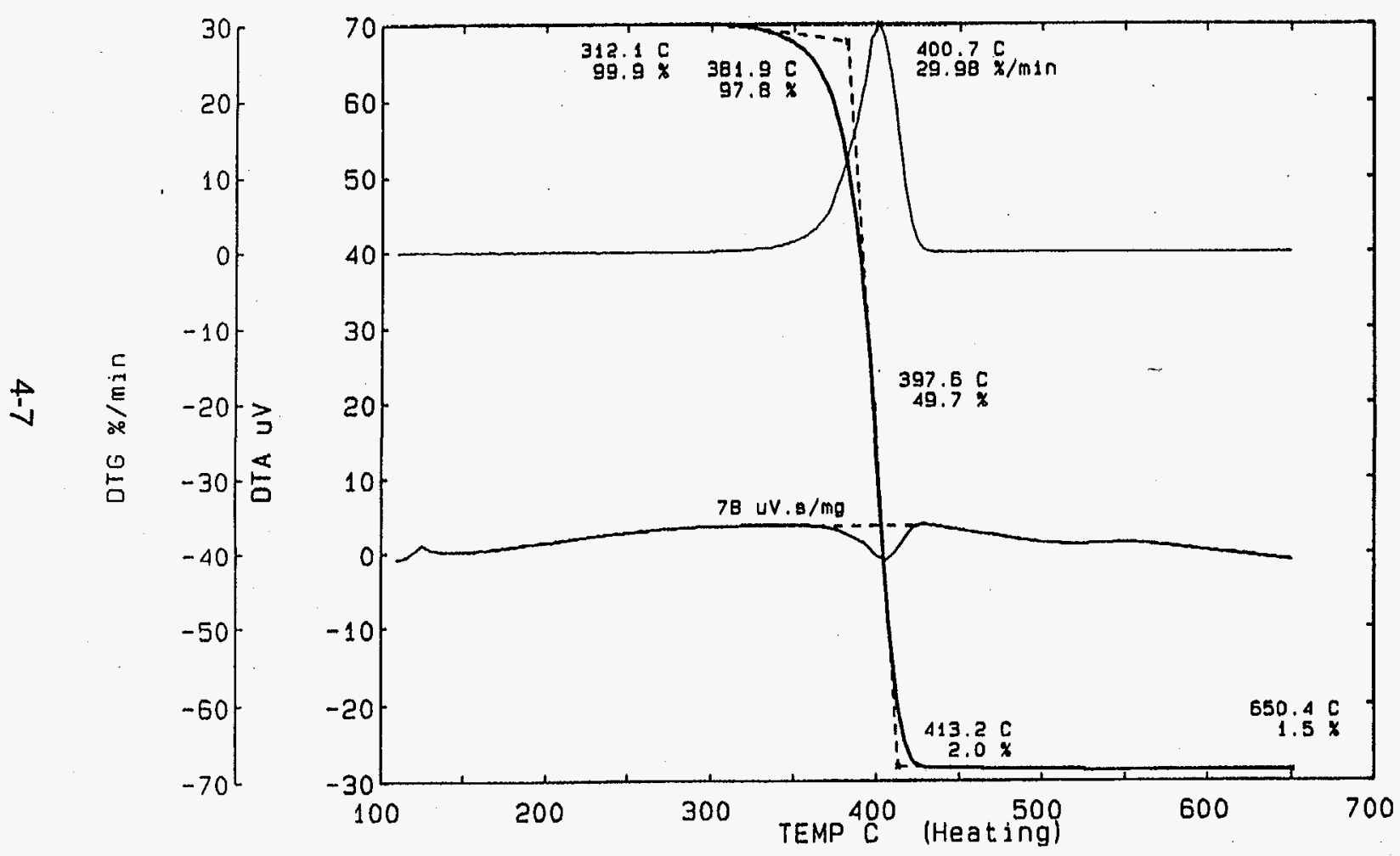

Polyethylene glycol

\begin{tabular}{|c|}
\hline Polyethylene glycol \\
\hline Heating rate- ${ }^{\circ} \mathrm{C} / \mathrm{min}: 10$ \\
\hline Sample weight-mg: 8.825 \\
\hline$T_{\text {thlual }} 0^{\circ} \mathrm{C}: 312.1$ \\
\hline Volatilization \\
\hline $\mathrm{T}_{\text {gun-podx- }}{ }^{-\mathrm{C}}: 381.9$ \\
\hline$T_{\text {mulpotax }} x^{\circ} \mathrm{C}: 397.6$ \\
\hline$T_{\text {add podex }}{ }^{\circ} \mathrm{C}: 413.2$ \\
\hline$T_{\max } x^{\circ} \mathrm{C}: 400.7$ \\
\hline Rate $_{\max }-\mathrm{mg} / \mathrm{min}: 29.98$ \\
\hline Wt. \% aso c $: 1.5$ \\
\hline$\Delta \mathrm{H}_{\mathrm{vol},}, \mu \mathrm{v}, \mathrm{sec} / \mathrm{mg}_{\mathrm{g}}: 78$ \\
\hline Proximate analysis \\
\hline Volatiles Tout $-w t \%: 98.5$ \\
\hline Volatiles oso $c^{-w}$ t\%: 98.5 \\
\hline Volatiles \\
\hline Fixed carbon-wt\%: 1.3 \\
\hline Ash-wt\%: 0.2 \\
\hline
\end{tabular}



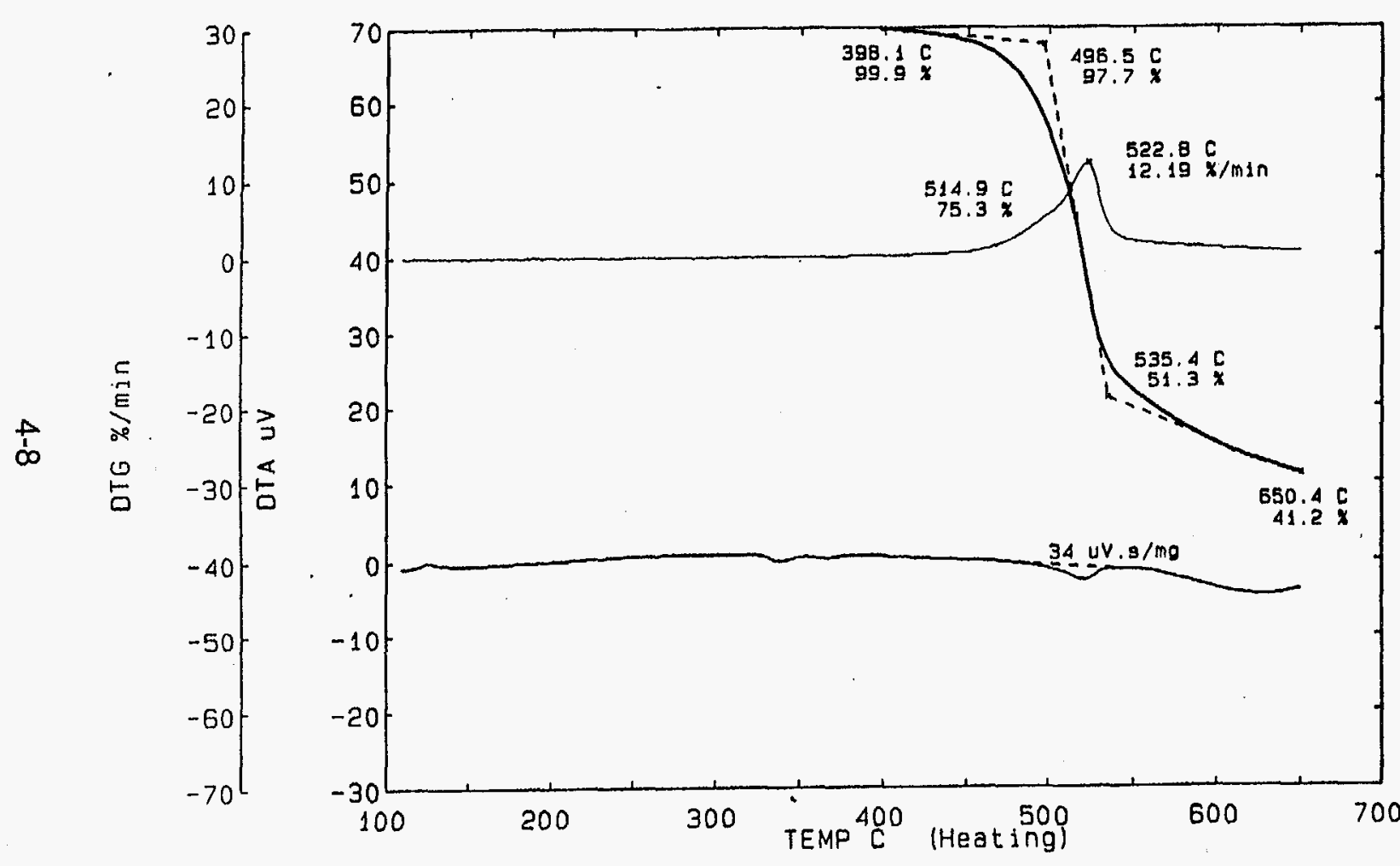

100

90

BO

70

60

50

x

$40 \stackrel{\circ}{\leftarrow}$

30

20

10

0

Polyhydroxy benzoic acid

\begin{tabular}{|c|}
\hline Polyhydroxy benzoic acid \\
\hline Heating rate- ${ }^{\circ} \mathrm{C} / \mathrm{min}: 10$ \\
\hline Samplo weight-mg: 6.918 \\
\hline $\mathrm{T}_{\text {minut }}{ }^{-\mathrm{C}} \mathrm{C}: 398.1$ \\
\hline Volatilization \\
\hline$T_{\text {and.polax }}{ }^{\circ} \mathrm{C}: 496.5$ \\
\hline$T_{\text {matpolex }}{ }^{\circ} \mathrm{C}: 514.9$ \\
\hline $\mathrm{T}_{\text {exd.potax }}{ }^{-\mathrm{C}} \mathrm{C}: 535.4$ \\
\hline$T_{\max }-^{\circ} \mathrm{C}: 522.8$ \\
\hline Rate $_{\max }-\mathrm{mg} / \mathrm{min}: 12.19$ \\
\hline Wt. \% a c c: 41.2 \\
\hline$\Delta \mathrm{H}_{\text {vol. }}-\mu \mathrm{v}: \mathrm{sec} / \mathrm{mg}: 34$ \\
\hline Proximate analysis \\
\hline 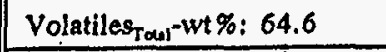 \\
\hline Volatiles oso $c^{-w t} \%: 58.8$ \\
\hline Volatiles ssa-sso c-wt\%: 5.8 \\
\hline Fixed carbon-wt\%: 35.4 \\
\hline Ash-wt\%: 0.0 \\
\hline
\end{tabular}



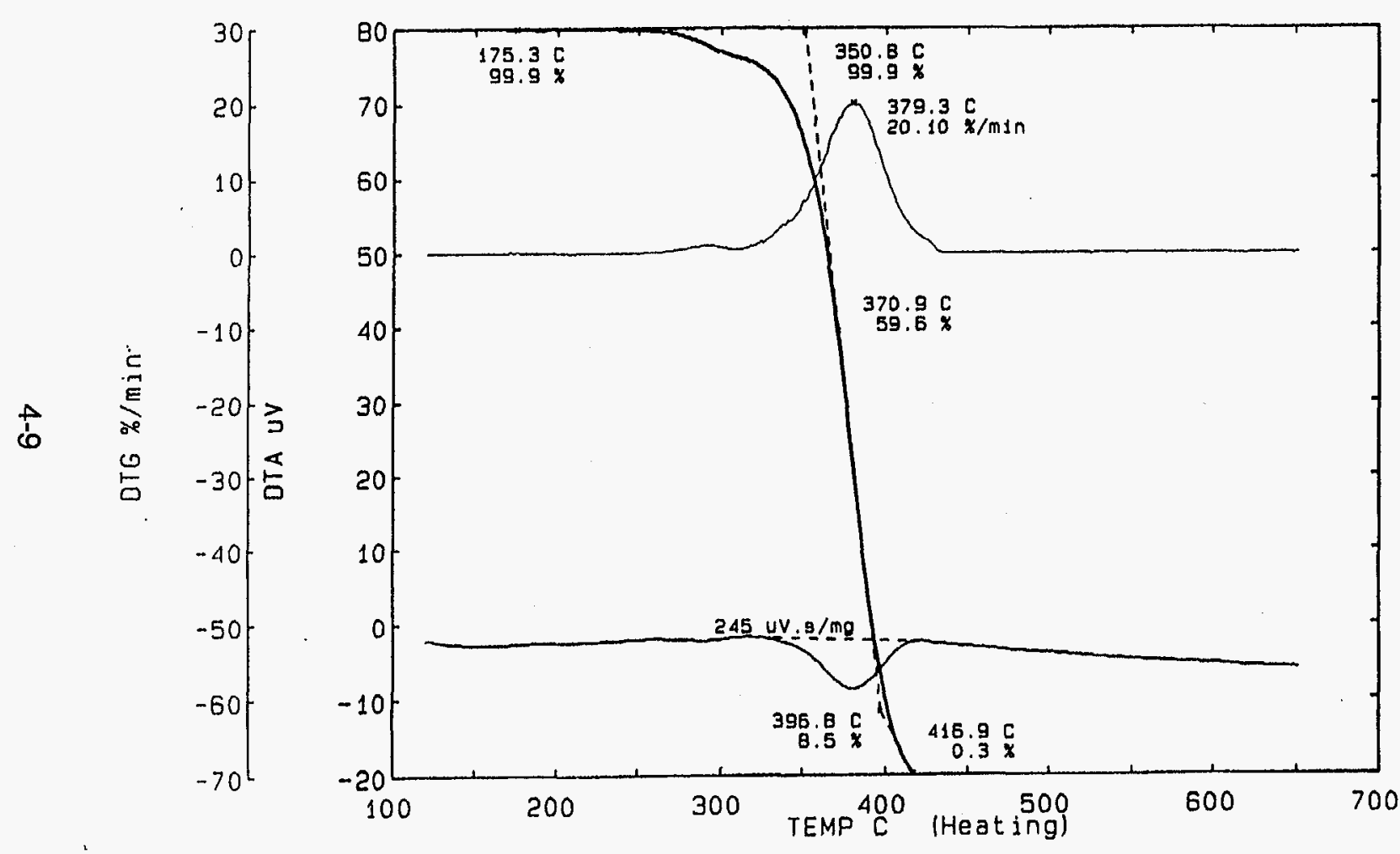

100

90

Bo

70

60

50

$x$

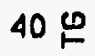

30

20

10

0

Polymethyl methacrylate

\begin{tabular}{|c|}
\hline Polymethyl methacrylate \\
\hline Heating rato- $0^{\circ} \mathrm{C} / \mathrm{min}: 10$ \\
\hline Sample weight-mg: 4.457 \\
\hline 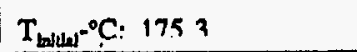 \\
\hline Volatilization \\
\hline$T_{\text {aun polat }}{ }^{\circ} \mathrm{C}: 350.8$ \\
\hline$T_{\text {mil-poldx }}{ }^{-0} \mathrm{C}: 370.9$ \\
\hline$T_{\text {end-podx }}$ - $^{\circ} \mathrm{C}: 396.8$ \\
\hline$T_{\max }{ }^{\circ} \mathrm{C}: 379.3$ \\
\hline Rate $_{\max } \cdot \mathrm{mg} / \mathrm{min}: 20.10$ \\
\hline Wt. $\%_{0000} c^{-:} 0.0$ \\
\hline$\Delta H$ vol. $-\mu ;$ v.sec/mg: NS \\
\hline Proximate analysis \\
\hline Volatiles ${ }_{\text {Toul }}-w t \%: 100.0$ \\
\hline Volatiles $_{s s 0} c^{-w t \%:} 100.0$ \\
\hline Volatiles $_{\triangle 50-950} c^{-w t \%: 0.0}$ \\
\hline Fixed carbon-wt $\%: 0.0$ \\
\hline Ash-wt\%: 0.0 \\
\hline
\end{tabular}




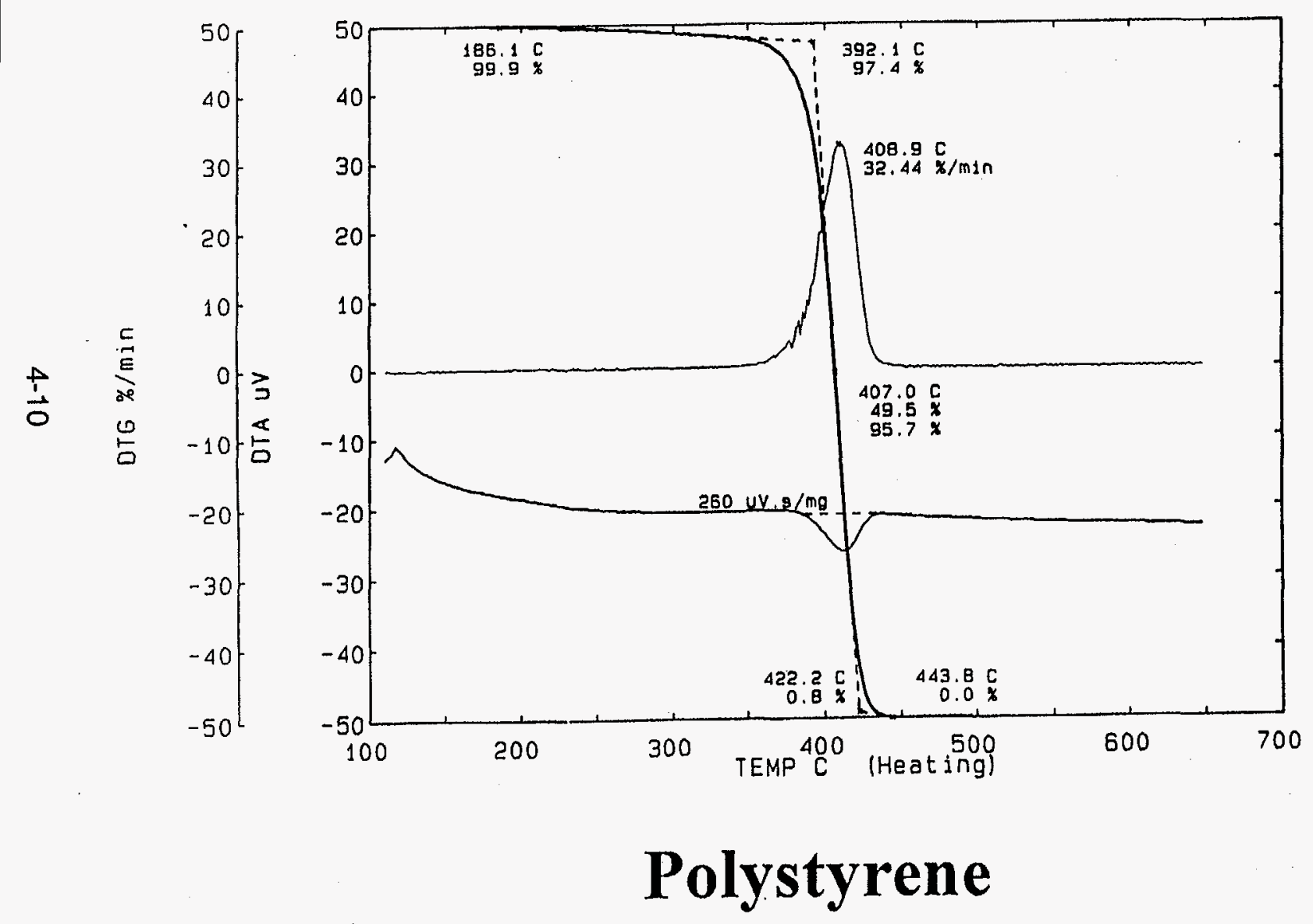

\begin{tabular}{|c|c|}
\hline 100 & Polystyrene \\
\hline 90 & \\
\hline & Heating rate- $-^{\circ} \mathrm{C} / \mathrm{min}: 10$ \\
\hline 80 & Sample weight-mg: 1.876 \\
\hline 70 & $\mathrm{~T}_{\text {isllhe }}--^{\circ} \mathrm{C}: 186.1$ \\
\hline & Volatilization \\
\hline ou & $T_{\text {sunn-polmx }}{ }^{-0} \mathrm{C}: 392.1$ \\
\hline 50 & $T_{\text {mbl-polot }}{ }^{-} \mathrm{C}: 407.0$ \\
\hline$x$ & $T_{\text {odd-polat }}{ }^{\circ}{ }^{\circ}: 443.8$ \\
\hline $40 \%$ & $\mathrm{~T}_{\operatorname{mex}}{ }^{\circ} \mathrm{C}: 408.9$ \\
\hline 30 & Rate $_{\max }-\mathrm{mg} / \mathrm{min}: 32.44$ \\
\hline & Wt. \% aso $c^{-:}: 0.0$ \\
\hline 20 & $\Delta H_{\text {vol. }}-\mu \mathrm{v} . \sec / \overline{m g}: 260$ \\
\hline 10 & Proximate analysis \\
\hline & Volatiles $_{\text {Toul }}-\mathrm{wt} \%: 100.0$ \\
\hline 0 & Volatiles sos c-w1\%: 100.0 \\
\hline & Volatiles ${ }_{650-950} c^{-w t} \%: 0.0$ \\
\hline & Fixed carbon-wt\%: 0.0 \\
\hline & Ash-wt\%: 0.0 \\
\hline
\end{tabular}




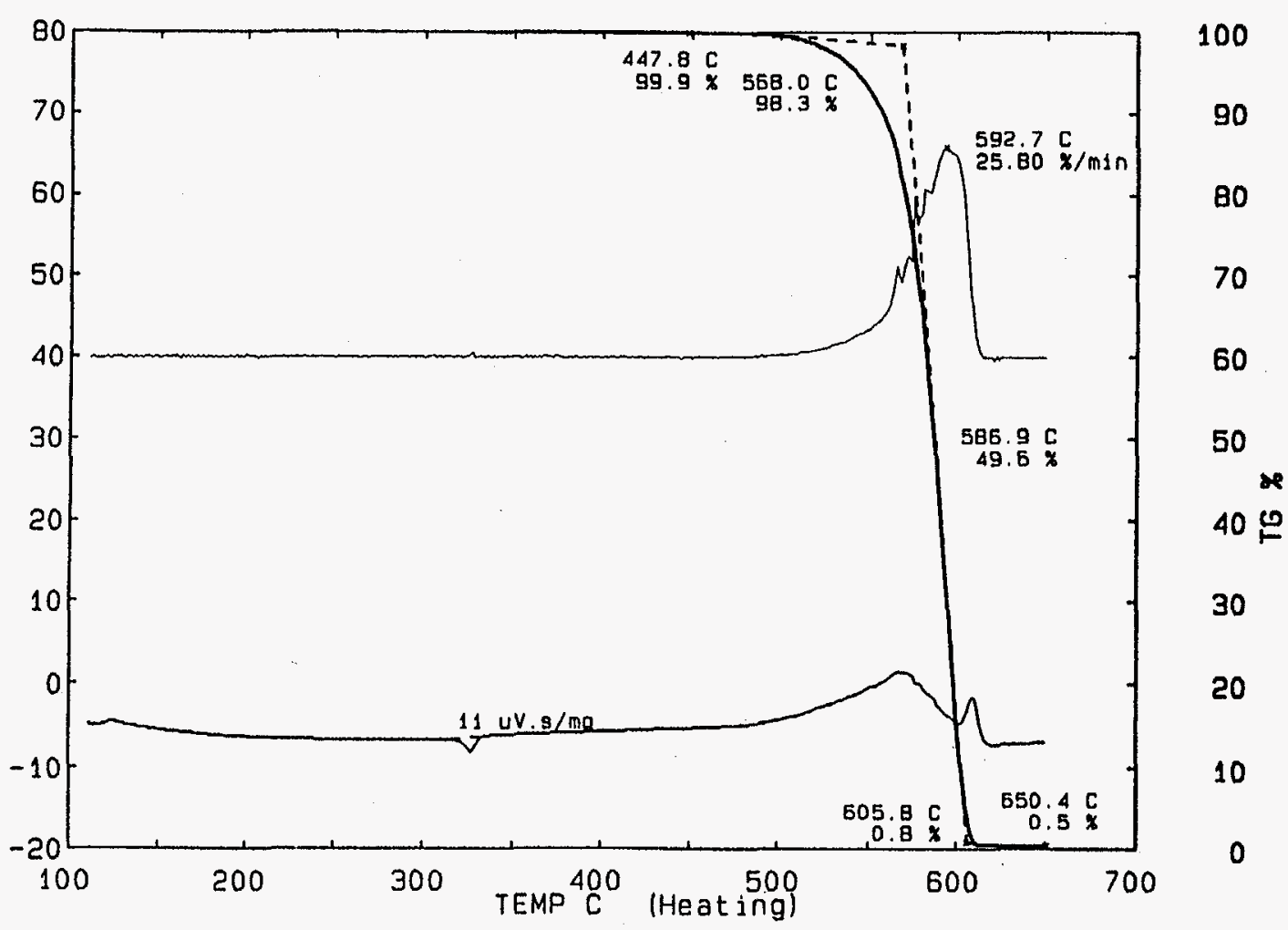

\section{Polytetraflouroethylene} (Teflon)

\begin{tabular}{|c|}
\hline Polytetraflouroethylene \\
\hline (Teflon) \\
\hline Heating rate- ${ }^{\circ} \mathrm{C} / \mathrm{min}: 10$ \\
\hline Sample weight-mg: 3.076 \\
\hline $\mathrm{T}_{\text {intatat }}{ }^{\circ} \mathrm{C}: 447.8$ \\
\hline Volatilization \\
\hline$T_{\text {sun:plax }}{ }^{\circ} \mathrm{C}: 568.0$. \\
\hline$T_{m b-p o l m}={ }^{\circ} \mathrm{C}: 586.9$ \\
\hline$T_{\text {exdpolx }}{ }^{\circ} \mathrm{C}: 605.8$ \\
\hline$T_{\max }{ }^{\circ} \mathrm{C}: 592.7$ \\
\hline Rate $_{\max }-\mathrm{mg} / \mathrm{min}: 25.8$ \\
\hline$W_{t . \%} \%$ sos c $: 0.5$ \\
\hline$\Delta \mathrm{H}_{\mathrm{vol}-}-\mu \mathrm{v} . \mathrm{sec} / \mathrm{mg}:$ \\
\hline Proximate analysis \\
\hline Volatiles $_{\text {Towe }}-w t \%: 100.0$ \\
\hline Volatiles ${ }_{\text {aso }} \mathrm{c}^{-\mathrm{wt} \%:} 99.5$ \\
\hline Volatiles ssa-9so $c^{-W t \%: 0.5}$ \\
\hline Fixed carbon-wt \%: 0.0 \\
\hline Ash-wt\%: 0.0 \\
\hline
\end{tabular}



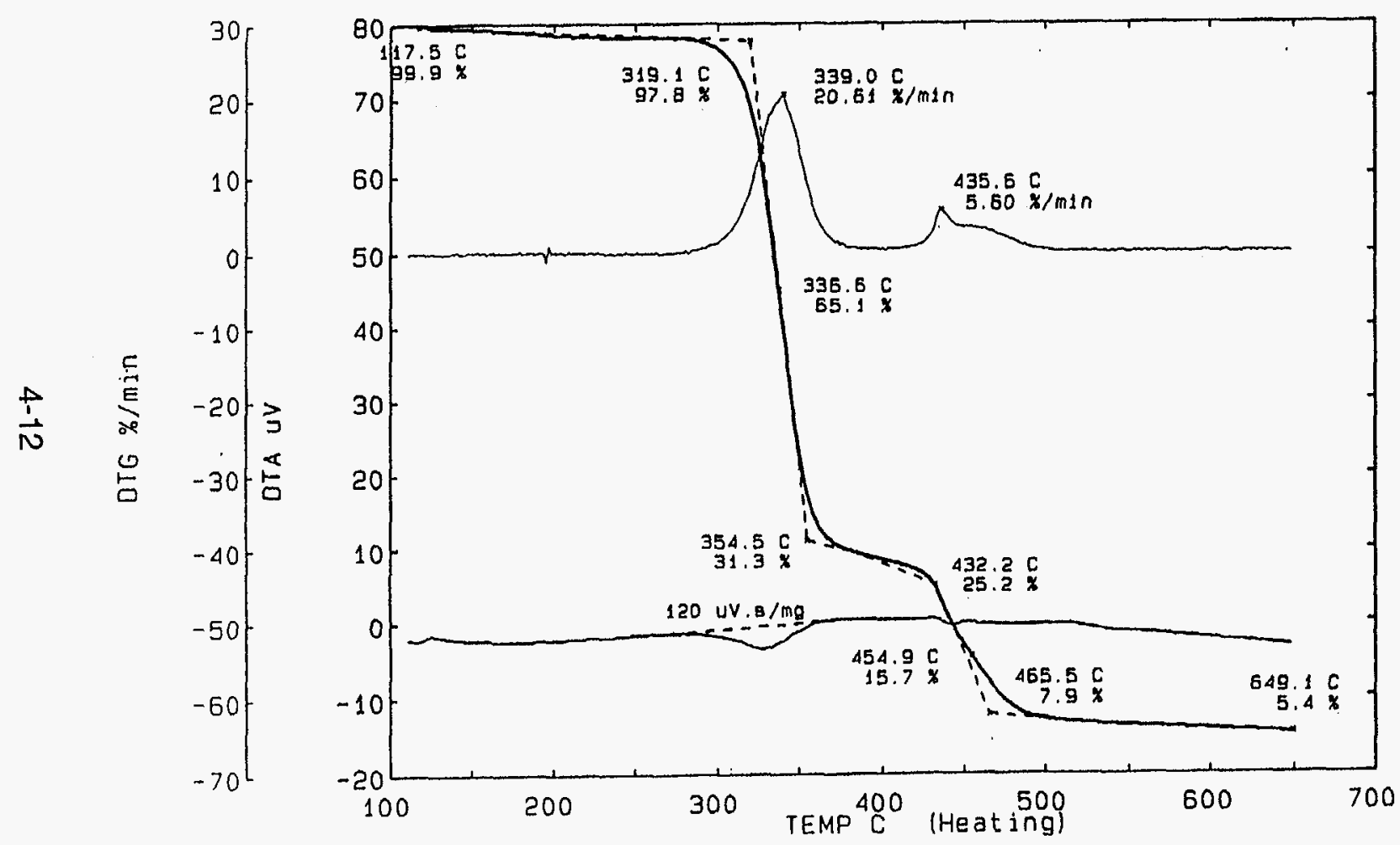

100

90

Polyvinyl acetate

\begin{tabular}{|c|}
\hline Polyvinyl acetate \\
\hline Heating rate- ${ }^{-} \mathrm{C} / \mathrm{min}: 10$ \\
\hline Samplo weight-mg: 2.121 \\
\hline$T_{\text {Indwat }}{ }^{\circ} \mathrm{C}: 117.5$ \\
\hline Volatilization \\
\hline $\mathrm{T}_{\text {aur-polax }}{ }^{\circ} \mathrm{C}: 319.1$ \\
\hline$T_{\text {mbipolent }}{ }^{\circ} \mathrm{C}: 336.6$ \\
\hline$T_{\text {sedpoptex }} 0^{\circ} \mathrm{C}: 354.5$ \\
\hline$T_{\max } x^{\circ} \mathrm{C}: 339.0$ \\
\hline Rate $_{\operatorname{mx}}-\mathrm{mg} / \mathrm{min}: 20.61$ \\
\hline Wt. $\%_{\text {oso } c^{-}: 5.4}$ \\
\hline$\Delta H$ vol. $-\mu ;$ v.sec/mg: 120 \\
\hline Proximate analysis \\
\hline Volatiles $_{\text {Toul }}-w t \%: 94.6$ \\
\hline Volatiles $_{s s 0} c^{-w t} \%: 94.6$ \\
\hline Volatiles $_{30,950} c^{-w t} \%: 0.0$ \\
\hline Fixed carbon-wt\%: 5.4 \\
\hline Ash-wt \%: 0.0 \\
\hline
\end{tabular}




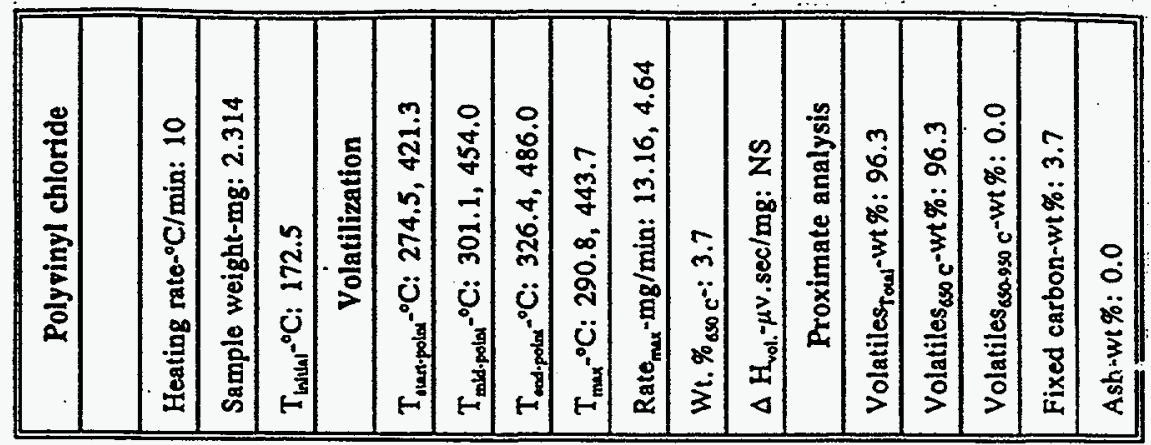

$$
\text { × } 91
$$

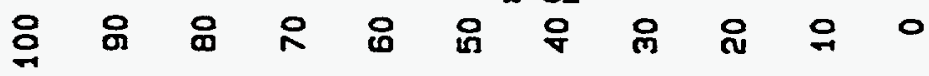

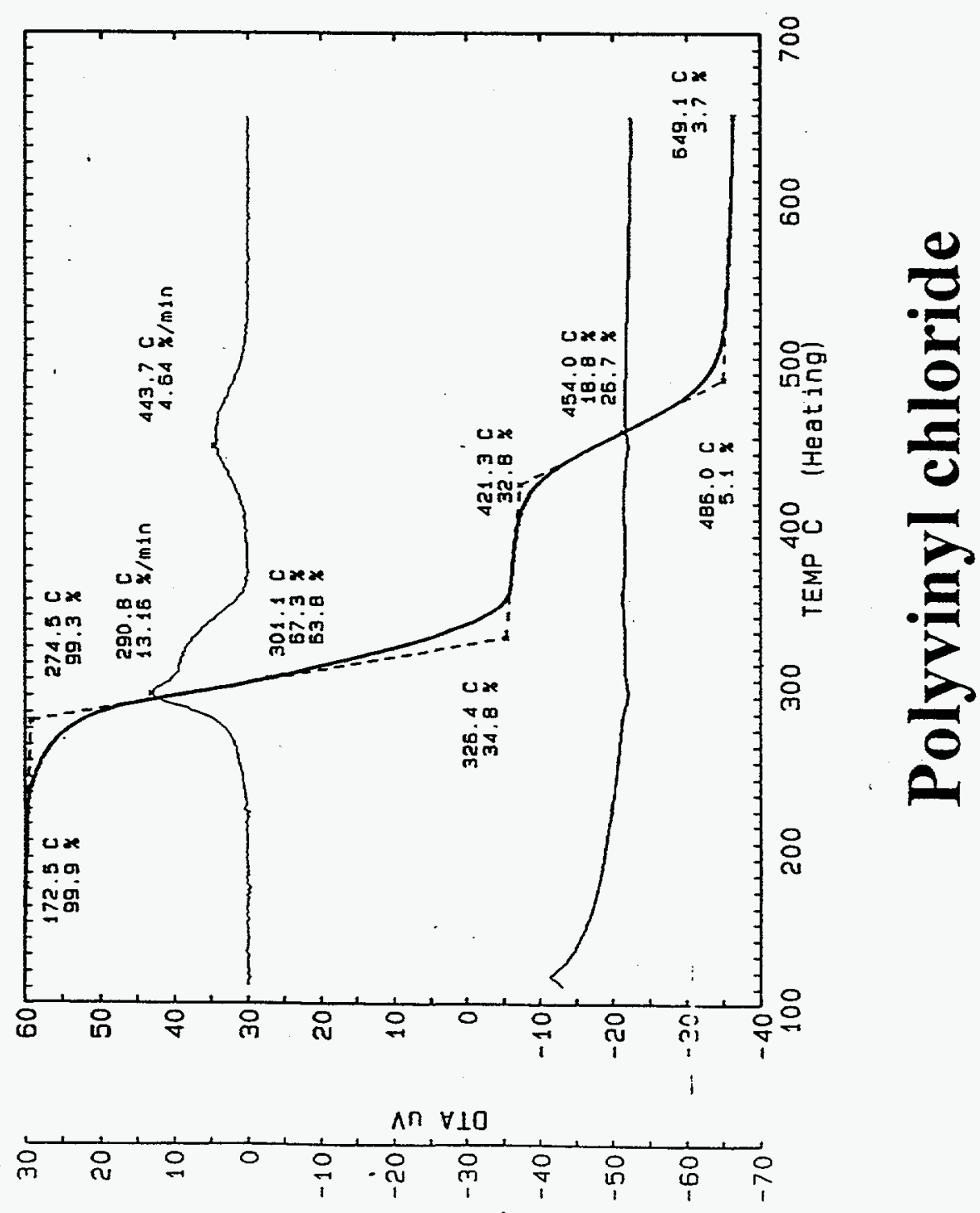

$u \underline{u / \%} 910$ 


\section{Chapter 5}

\section{HIGH CARBON SOLID FUELS}

\subsection{Charcoal}

If wood and other biomass materials are considered as humankind's earliest fuel, then the oldest synthetic fuel is charcoal. The use of wood for fuel produces a smoky low efficiency fire, unless it is burned in well designed stoves and furnaces. On the other hand charcoal produces a very clean and hot fire, needed for indoor cooking, metal working and now used in many chemical processes. Conversion of wood to charcoal is a very old art. In early times charcoal was made by the slow heating of wood in the absence of air [Earl, 1974]. More recently processes have been developed to make pyrolysis oils and charcoal simultaneously [Diebold, 1988, Richard, 1994].

The name charcoal does not begin adequately to describe the hundreds of varieties of materials in commercial use. One simple index of charcoals is the percent of volatiles remaining after pyrolysis. A very high volatile charcoal called torrefied wood is produced in the range $230-270^{\circ} \mathrm{C}$. It is claimed that torrefied wood contains most of the fuel value of the original wood in a much higher concentration.

If wood is pyrolyzed in the range $300-360^{\circ} \mathrm{C}$, the hemicellulose is selectively destroyed, while the strength and cellular structure of the wood is preserved. This material is rendered hydrophobic and oleophilic and is an excellent absorbent for oils and chemicals marketed under the name Sea Sweep [Reed, 1994].

If wood is heated to about $300^{\circ} \mathrm{C}$ the reaction becomes exothermic and can go spontaneously to about $450^{\circ} \mathrm{C}$. This composition would represent the "cooking charcoal" still widely used around the world and responsible for much deforestation. While most easily made from wood, cooking charcoal can also be made from rice hulls, bagasse and other waste materials if it is briquetted. Such processing could reduce the deforestation now of concern.

Charcoal for metallurgical purposes typically is produced by heating above $450^{\circ} \mathrm{C}$. It has a very low sulfur content and is used in making high grade steel among other things.

Finally, if charcoal is heated to $800-900^{\circ} \mathrm{C}$, or chemically treated, an activated charcoal results which has a very high internal surface area. Typically only $20-40 \%$ of the original biomass remains, so that the density is very low. Activated charcoal is useful for absorbing large molecules, toxics, etc. Activated charcoals for liquid purification (i.e. sugar decolorizing) are used in small particle size. For gas purification, large particles are required and so the original biomass must be very dense in order to maintain physical structure for example samples like coconut hulls are good for this purpose. 
The thermograms of charcoal show the degree of volatiles still remaining and thus indicate the degree of charring.

\subsection{Coal}

Coal has been an excellent fuel for hundreds of years and provided the energy basis of the Industrial Revolution. Coal is produced from biomass by varying degrees of compression over the last billion years. A number of degrees of conversion exist from lignite, a soft brown coal, through bituminous to hard anthracite coal. The representative samples studied in this Atlas were obtained from the Argonne National Laboratory, Argonne, Illinois and illustrate the variability in volatiles, fixed carbon and ash [IGT, 1978].

\subsection{Peat}

Like charcoal, peat has been burned as a fuel and used for fertilizer for thousands of years. It is bumed commercially to produce power, particularly in Ireland and Finland. Peat is the remains of plants that have decomposed, usually under water in bogs. The peat must be dried before use. The International Humic Institute, located at the Colorado School of Mines, collects and analyses various forms of peat and has contributed the samples studied here [IHI, 1993]. 

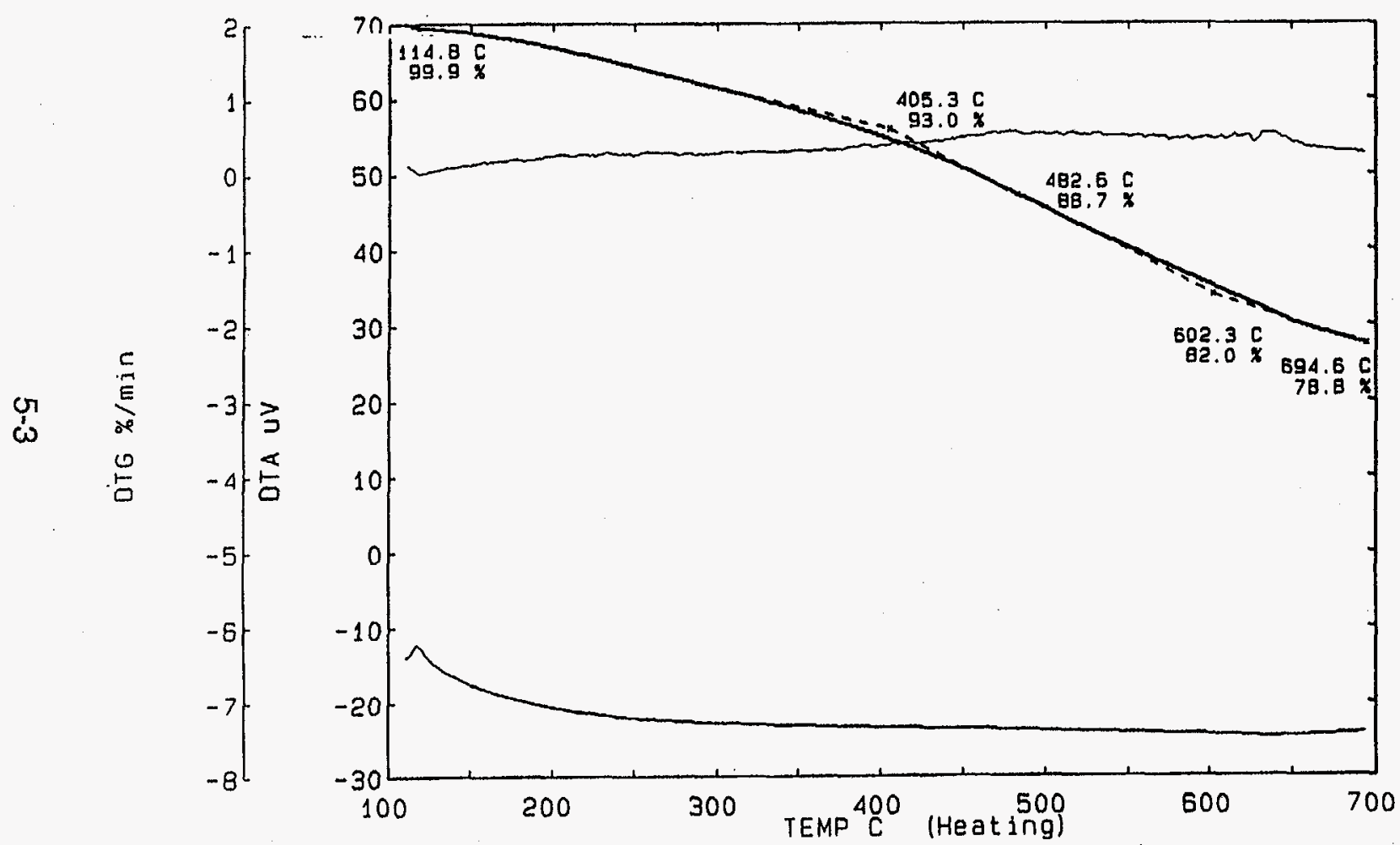

100
95
90
85
80
75
70
65
60
55
50

\section{Switchgrass char}

'(Panicum virgatum L.)

\begin{tabular}{|c|}
\hline $\begin{array}{c}\text { Switch grass char } \\
\text { (Panicum virgatum L }\end{array}$ \\
\hline Heating rate- ${ }^{\circ} \mathrm{C} / \mathrm{min}: 10$ \\
\hline Sample weight-mg: 3.595 \\
\hline $\mathrm{T}_{\text {|lduat }}{ }^{\circ} \mathrm{C}: \mathrm{114.8}$ \\
\hline Volatilization \\
\hline 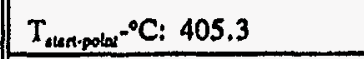 \\
\hline$T_{\text {mid-polut }}{ }^{\circ} \mathrm{C}: 482.6$ \\
\hline $\mathrm{T}_{\text {codspolax }}{ }^{\circ} \mathrm{C}: 602.3$ \\
\hline$T_{\text {max }}-{ }^{\circ} \mathrm{C}: N S$ \\
\hline $\mathrm{Rate}_{\max }-\mathrm{mg} / \min : \mathrm{NS}$ \\
\hline Wt. \% oso c: 78.8 \\
\hline$\Delta \mathrm{H}_{\text {vol. }}-\mu \mathrm{v} . \mathrm{sec} / \mathrm{mg}: \mathrm{NS}$ \\
\hline Proximate analysis \\
\hline Volatiles $_{\text {Toul }}-$ wi \%: 26.5 \\
\hline Volatiles $\operatorname{soc} c^{-w t \%: 21.2}$ \\
\hline Volatiles, ${ }_{9 s 0} c^{-w t} \%: 5.3$ \\
\hline Fixed carbon-wt\%: 47.8 \\
\hline Ash-wt\%: 25.7 \\
\hline
\end{tabular}



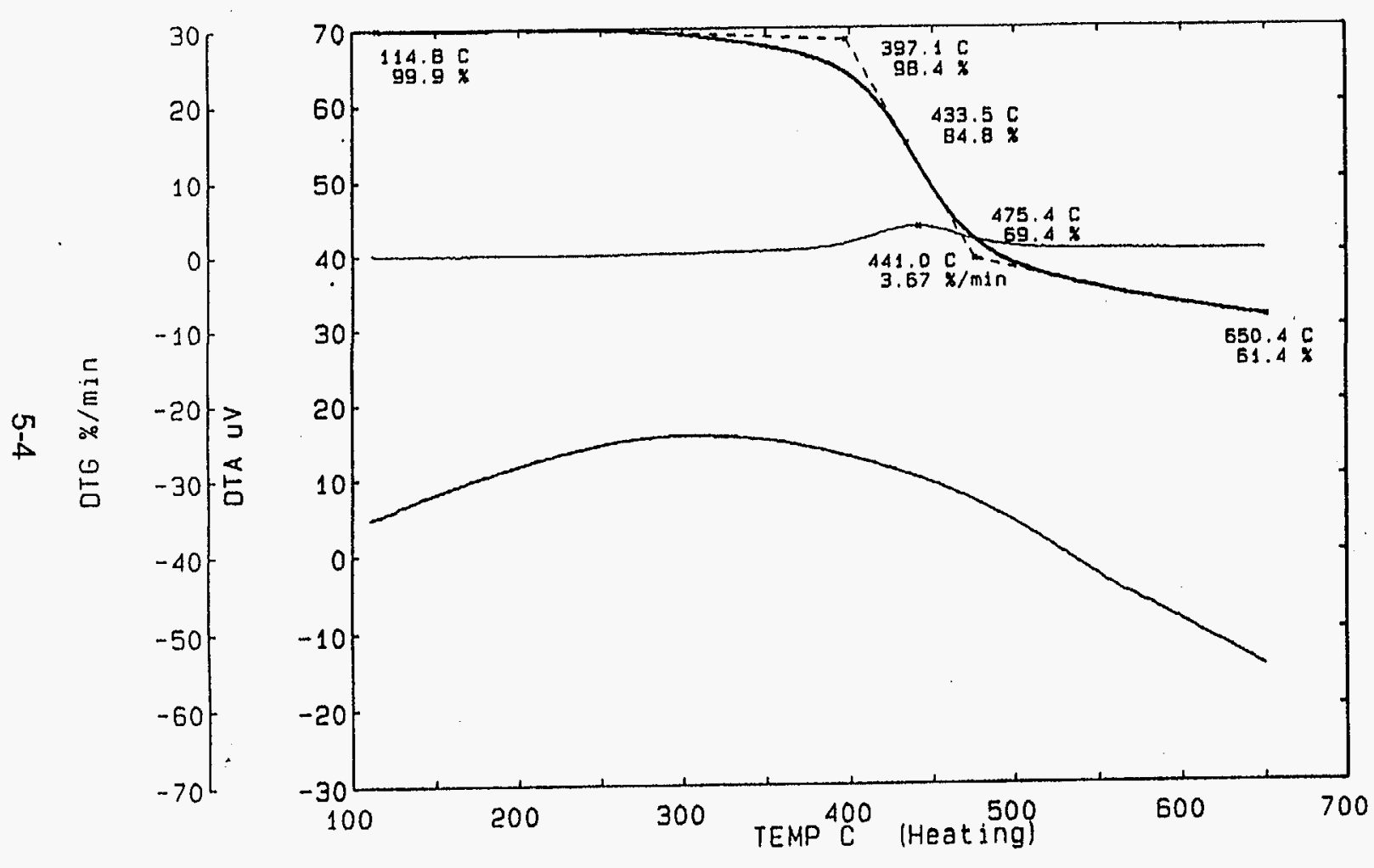

\begin{tabular}{|c|c|}
\hline & \\
\hline 90 & $\begin{array}{l}\text { Blind canyon coal } \\
\text { (High volatile bituminous\}) }\end{array}$ \\
\hline $\mathrm{PO}$ & Heating rate- ${ }^{\circ} \mathrm{C} / \mathrm{min}: 10$ \\
\hline & Sample weight-mg: 7.258 \\
\hline 70 & $T_{\text {bldstat }} 0^{\circ} \mathrm{C}: \quad 114.8$ \\
\hline 60 & Volatilization \\
\hline & $\mathrm{T}_{\text {ann.polex }}{ }^{-0} \mathrm{C}: 397.1$ \\
\hline 5 & $\mathrm{~T}_{\text {mit.polux }}{ }^{\circ} \mathrm{C}: 433.5$ \\
\hline$\infty$ & 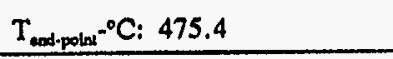 \\
\hline & $T_{\text {max }}{ }^{-0}: 441.0$ \\
\hline 30 & Rate $_{\max }-\mathrm{mg} / \mathrm{min}: 3.67$ \\
\hline 20 & Wt. \% oso c: 61.4 \\
\hline & $\Delta \mathrm{H}_{\mathrm{vol}}-\mu \mathrm{v}, \mathrm{sec} / \mathrm{mg}: \mathrm{NS}$ \\
\hline 10 & Proximate analysis \\
\hline 0 & Volatiles $_{\text {Tow }^{-}-w t \%: 45.1}$ \\
\hline & Volatiles $_{s 00} c^{-w t} \%: 38.6$ \\
\hline & Volatiles ${ }_{s s a .950} c^{-w t} \%: 6.5$ \\
\hline & Fixed carbon-wt\%: 50.9 \\
\hline & Ash-wt\%: 4.0 \\
\hline
\end{tabular}

\section{Coal, Blind canyon} (High volatile bituminous, Utah) 

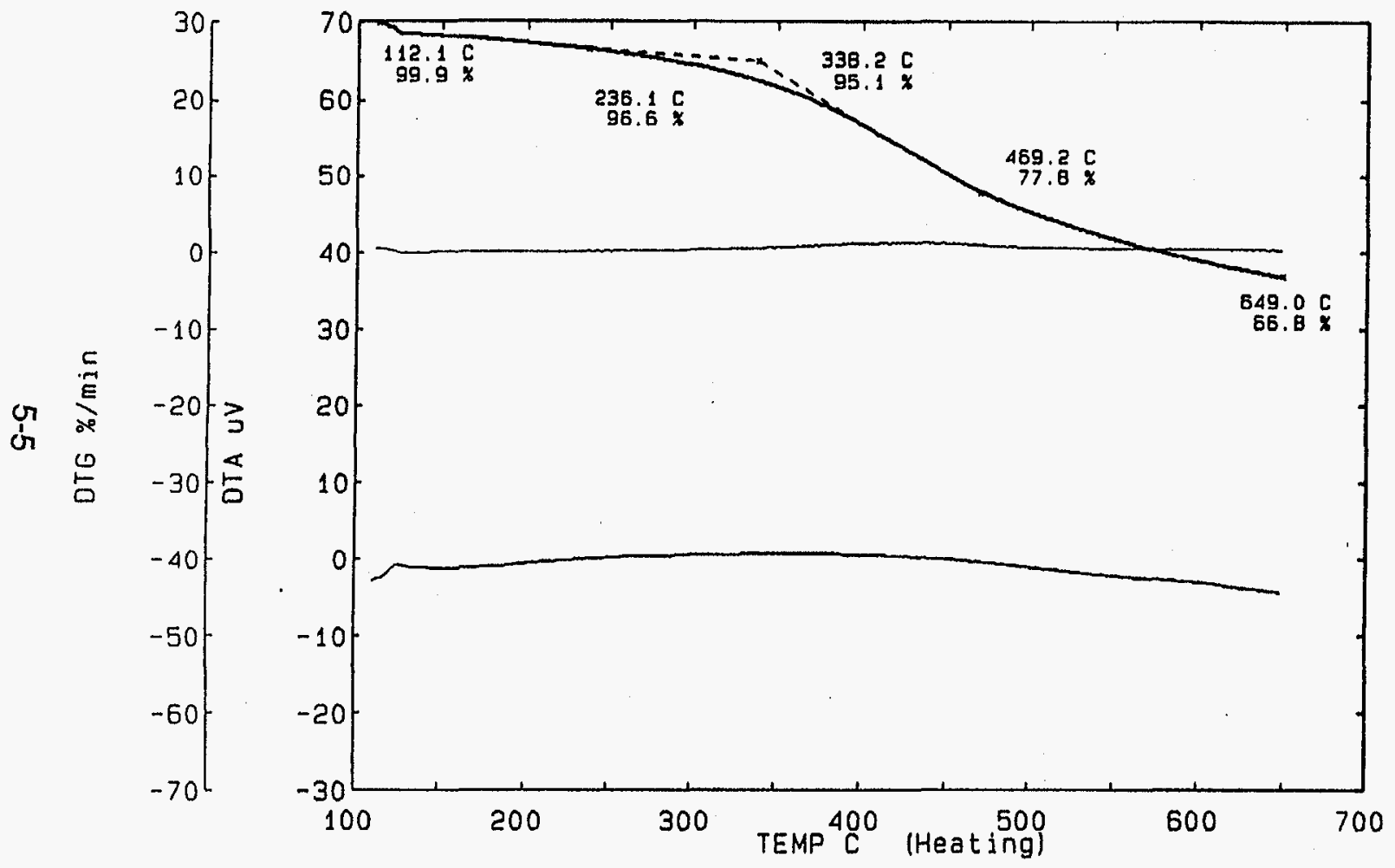

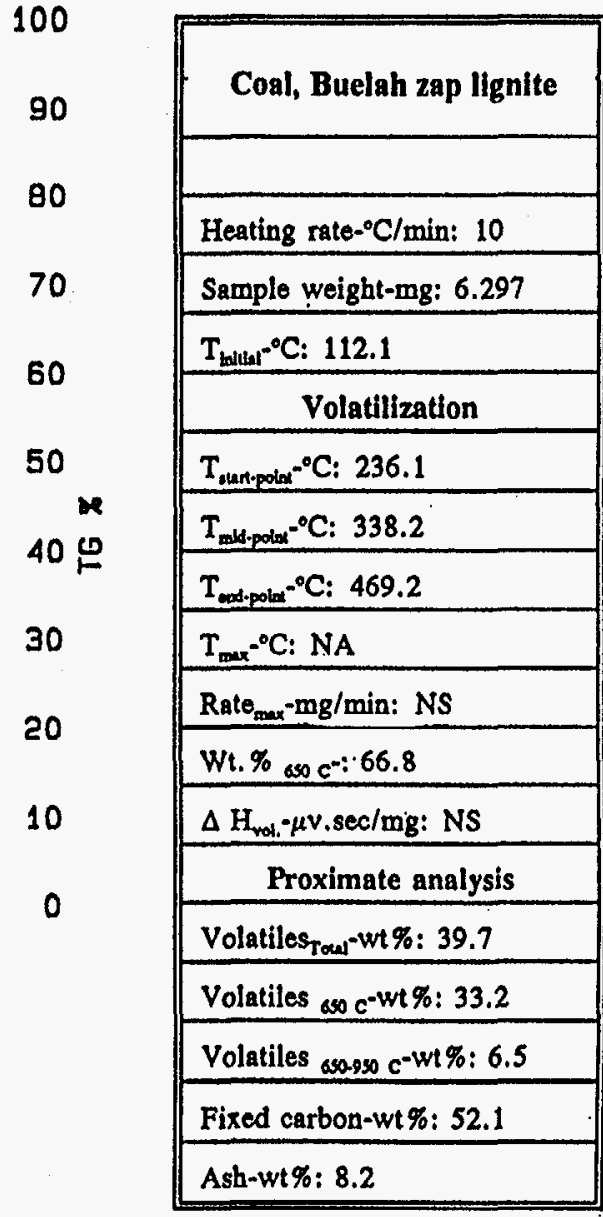

\section{Coal, Buelah zap lignite (North Dakota)}



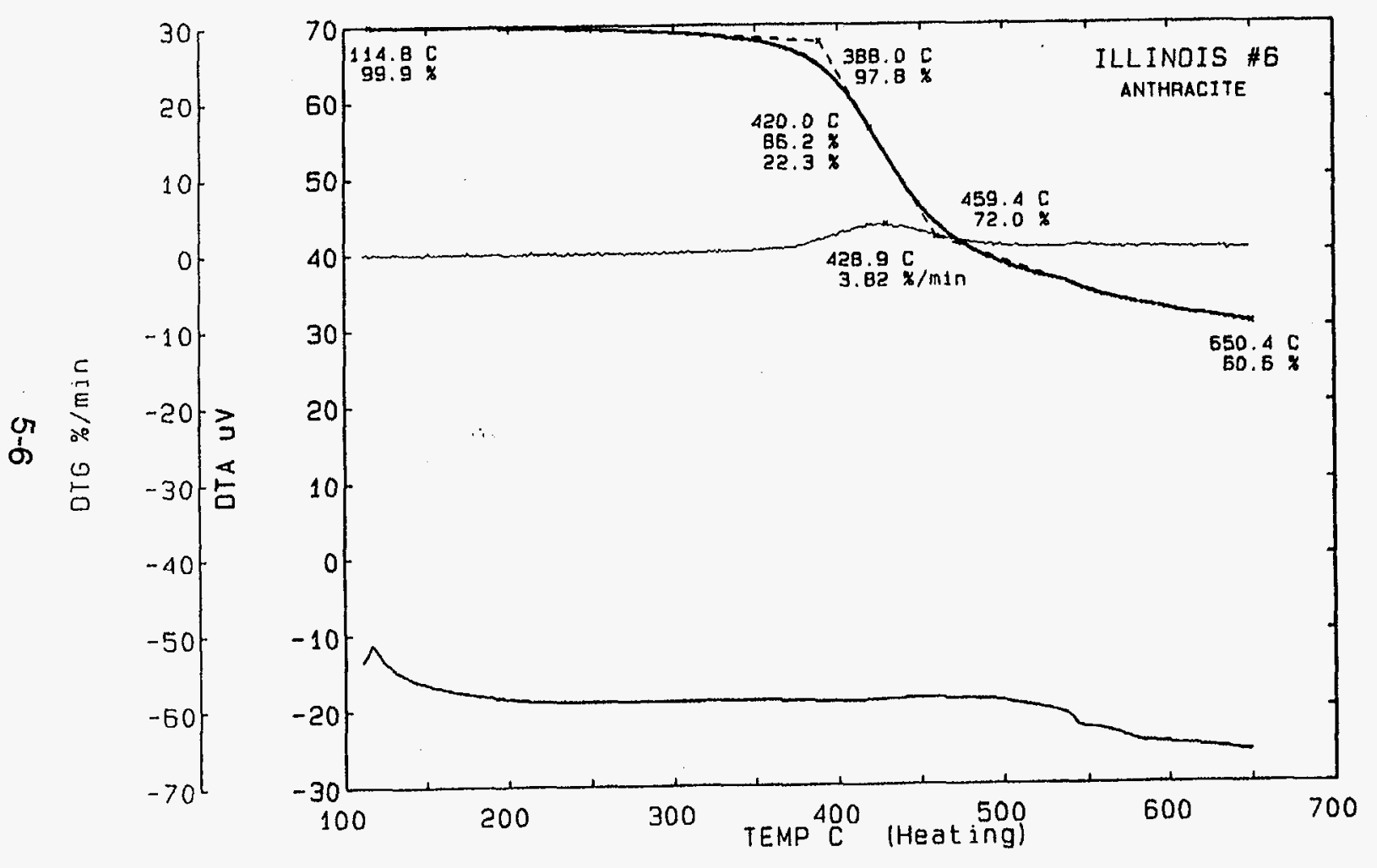

\begin{tabular}{|c|c|}
\hline & \\
\hline 90 & $\begin{array}{l}\text { Coal, Illinois \#6 } \\
\text { (High volatile bituminous }\end{array}$ \\
\hline 80 & Heating rate- ${ }^{\circ} \mathrm{C} / \mathrm{min}: 10$ \\
\hline & Sample weight-mg: 7.647 \\
\hline 70 & $\mathrm{~T}_{\text {inllat }\left.\right|^{\circ}}{ }^{\circ} \mathrm{C}: 114.8$ \\
\hline 50 & Volatilization \\
\hline & $\mathrm{T}_{\text {sunn-polm }}{ }^{\circ \mathrm{C}}: \quad 388.0$ \\
\hline 50 & $T_{\text {mblpoplext }}{ }^{\circ} \mathrm{C}: 420.0$ \\
\hline An & $\mathrm{T}_{\text {end-polnt }}{ }^{\circ} \mathrm{C}: 459.4$ \\
\hline & $\mathrm{T}_{\max }{ }^{\circ} \mathrm{C}: 428.9$ \\
\hline 30 & Rate $_{\text {mex }}-\mathrm{mg} / \mathrm{min}: 3.82$ \\
\hline ח & Wt. \% $\%_{0 s 0} c^{-:} 60.6$ \\
\hline & $\Delta H$ vol. - $\mu ; v . s e c / m g: ~ N S i$ \\
\hline 10 & Proximate analysis \\
\hline n & Volatiles $_{\text {Tout }}-w t \%: 40.6$ \\
\hline 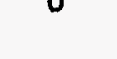 & Volatiles $_{\Delta s 0} c^{-w t} \%: 39.4$ \\
\hline & Volatiles $_{500.950} c^{-w t \%: ~} 1.2$ \\
\hline & Fixed carbon-w1\%: 43.9 \\
\hline & Ash-wl\%: 15.5 \\
\hline
\end{tabular}

Coal, Illinois \#6

(High volatile bituminous, Illinois) 


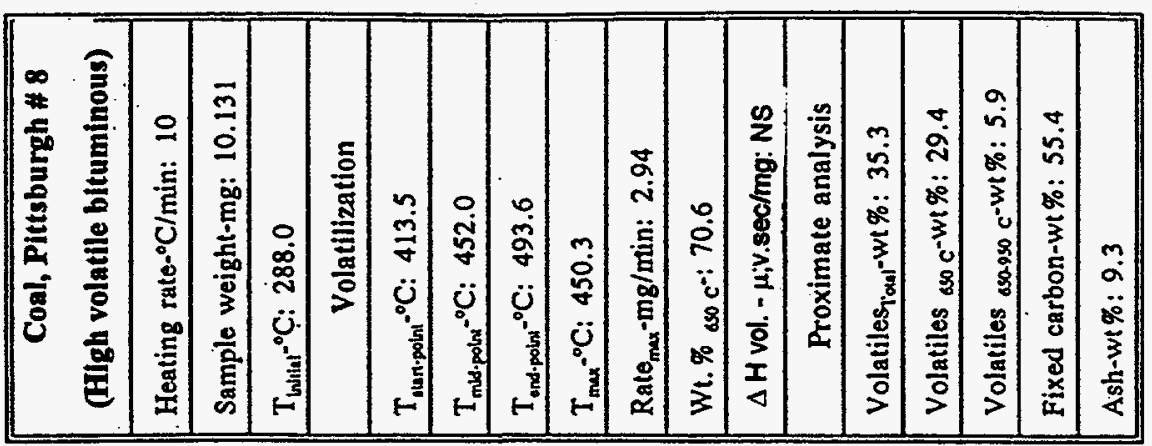

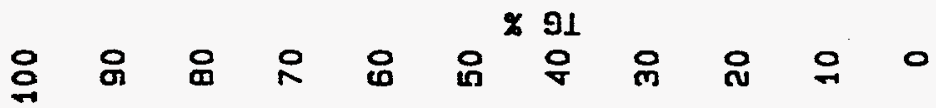
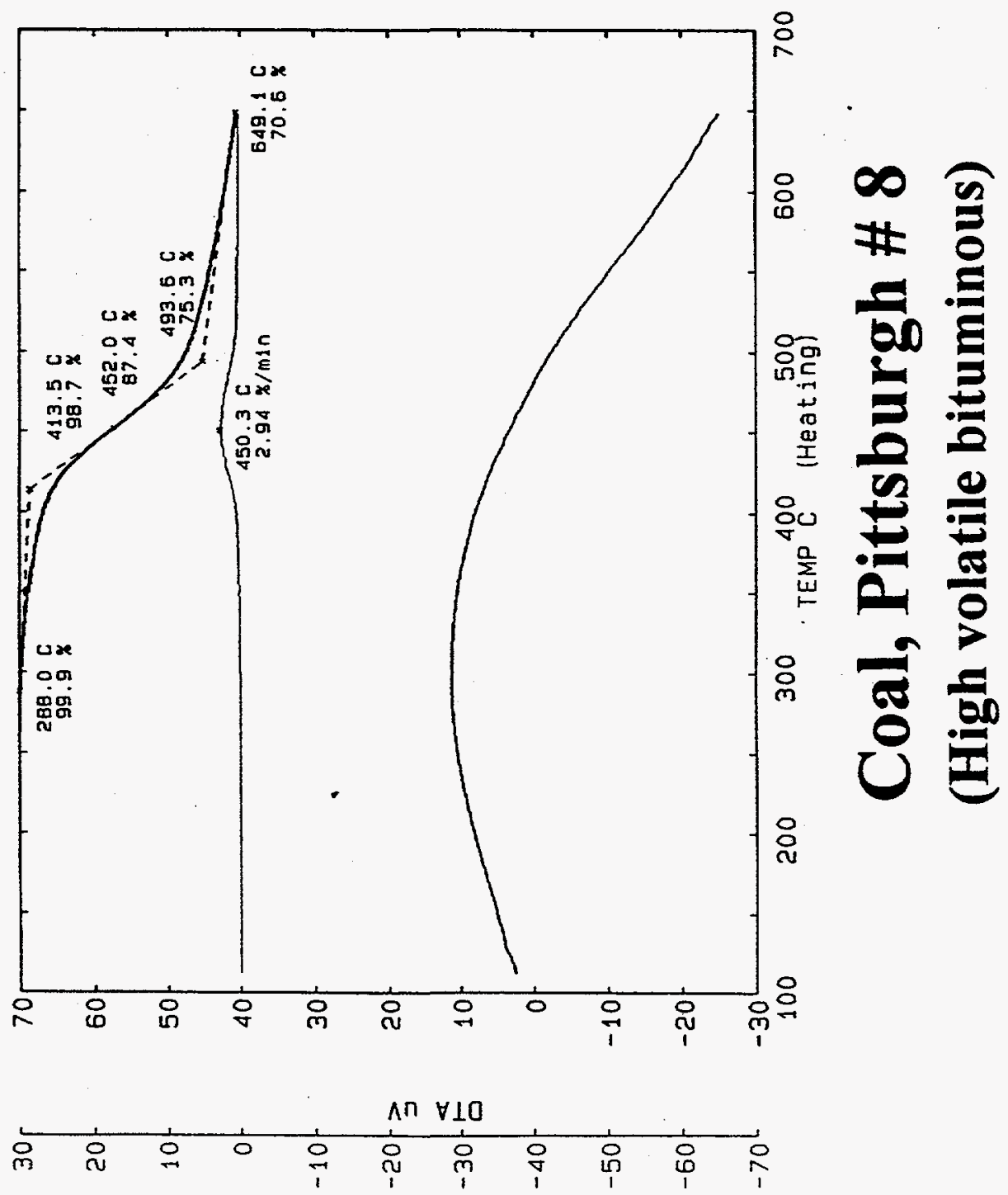

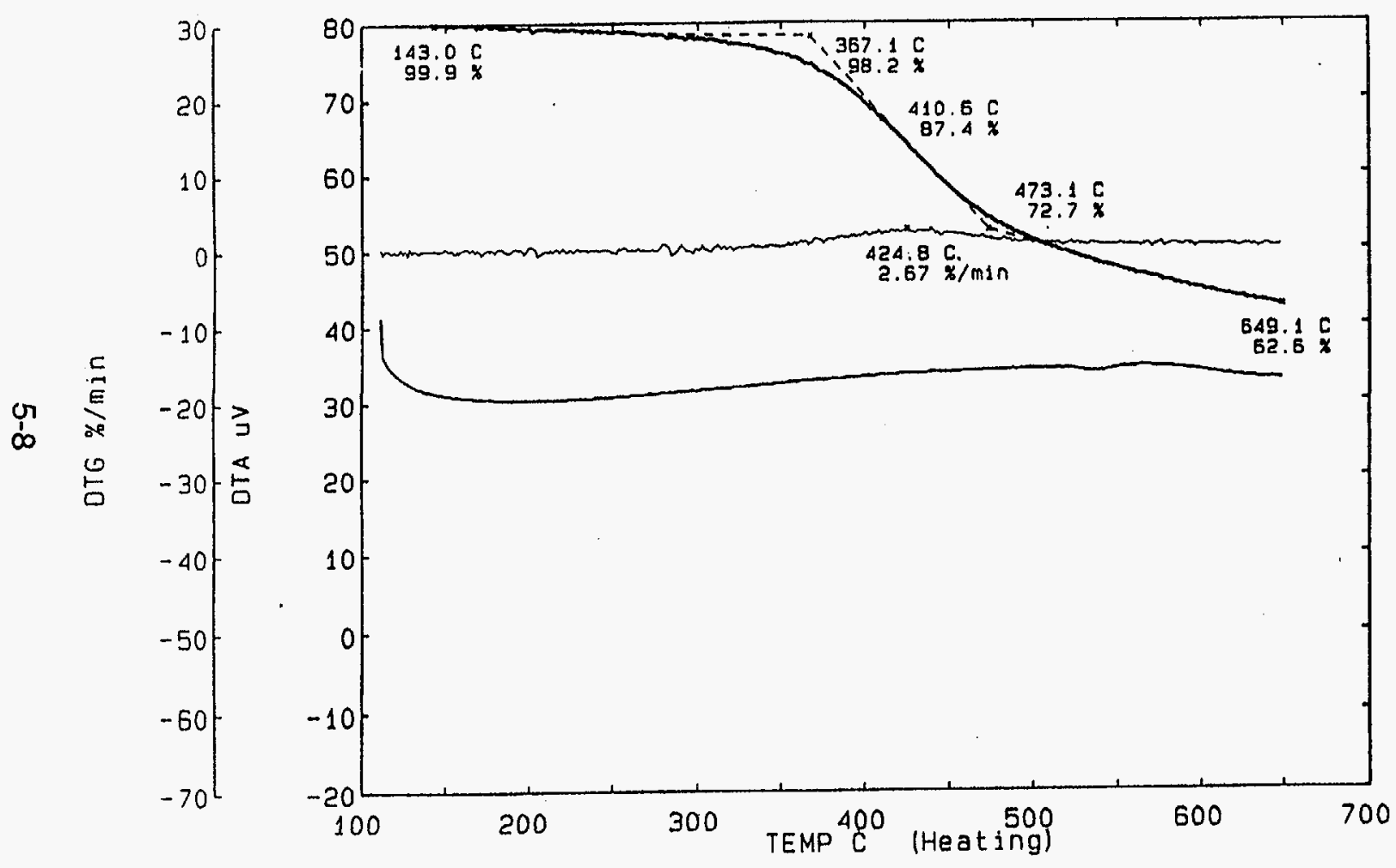

\begin{tabular}{|c|c|}
\hline 90 & $\begin{array}{l}\text { Coal, Wyodak bituminous } \\
\text { (Subbituminous, Wyoming) }\end{array}$ \\
\hline \multirow{2}{*}{80} & Heating rate- ${ }^{\circ} \mathrm{C} / \mathrm{min}: 10$ \\
\hline & Sample weight-mg: 7.06 \\
\hline 70 & $T_{\text {milutit }}{ }^{\circ} \mathrm{C}: 143.0$ \\
\hline \multirow{2}{*}{60} & Volatilization \\
\hline & $\mathrm{T}_{\text {sunnpolm }}{ }^{\circ} \mathrm{C}: 367.1$ \\
\hline 50 & $\mathrm{~T}_{\text {med-polnt }}{ }^{-0} \mathrm{C}: 410.6$ \\
\hline \multirow{2}{*}{40} & $T_{\text {ond.polex }}{ }^{\circ} \mathrm{C}: 473.1$ \\
\hline & $\mathrm{T}_{\max }{ }^{\circ} \mathrm{C}: 424.8$ \\
\hline 30 & Rate $_{\max }-\mathrm{mg} / \mathrm{min}: 2.67$ \\
\hline \multirow{2}{*}{20} & Wt. $\dot{\%}_{\Delta s 0} c^{-:}: 62.6$ \\
\hline & $\Delta H_{\mathrm{vol}}, \mu v . \sec / \mathrm{mg}: \mathrm{NS}$ \\
\hline 10 & Proximate analysis \\
\hline \multirow{5}{*}{0} & Volatiles $_{\text {Toxt }}-w 1 \%: 55.1$ \\
\hline & Volatiles ${ }_{\text {aso }} \mathrm{c}^{-w t} \%: 37.4$ \\
\hline & Volatiles $850.950 c^{-w t} \%: 17.7$ \\
\hline & Fixed carbon-wt\%: 37.9 \\
\hline & Ash-wt\%: 7.0 \\
\hline
\end{tabular}

- Coal, Wyodak bituminous (Subbituminous, Wyoming) 


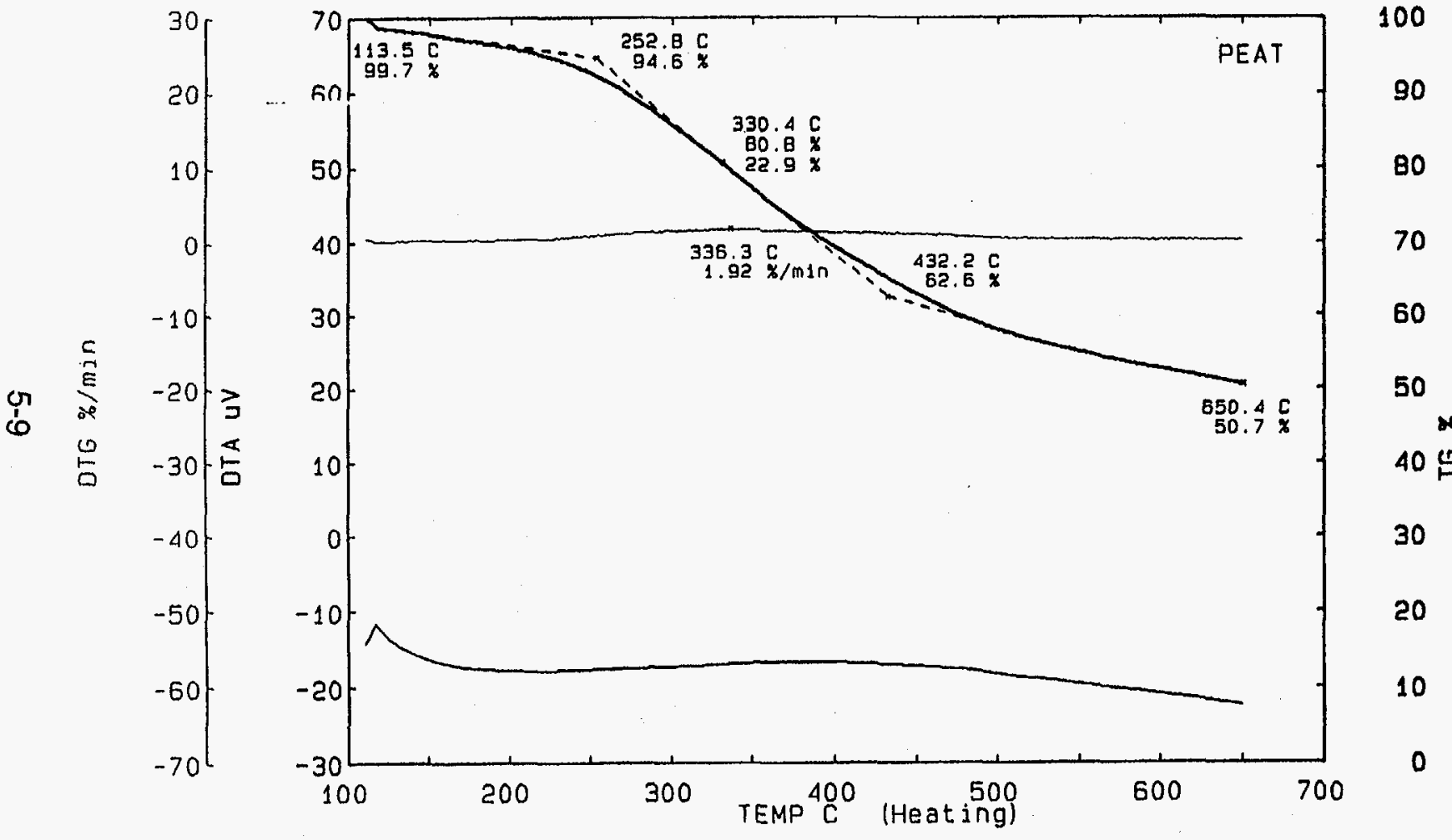

\begin{tabular}{|c|}
\hline $\begin{array}{c}\text { Peat } \\
\text { (Pahokee Polnt, Fla.) }\end{array}$ \\
\hline Heating rate- ${ }^{\circ} \mathrm{C} / \mathrm{min}: 10$ \\
\hline Sample weight-mg: 2.687 \\
\hline $\mathrm{T}_{\text {intitu- }}{ }^{-0} \mathrm{C}: 113.5$ \\
\hline Volatilization \\
\hline $\mathrm{T}_{\text {ular } \cdot \text { poln }}-^{\circ} \mathrm{C}: 252.8$ \\
\hline $\mathrm{T}_{\text {mid-point }} 0^{\circ} \mathrm{C}: 330.4$ \\
\hline $\mathrm{T}_{\text {end-point }}-^{\circ} \mathrm{C}: 432.2$ \\
\hline $\mathrm{T}_{\max }{ }^{\circ} \mathrm{C}: 336.3$ \\
\hline Rate $_{\max }-\mathrm{mg} / \min : 1.92$ \\
\hline Wt. $\%$ oss c c: 50.7 \\
\hline$\Delta H$ vol. - $\mu$;v.sec/mg: NS \\
\hline Proximate analysis \\
\hline Volatiles $_{\text {Tout }}-w t \%: 58.1$ \\
\hline Volatiles $_{6 s 0} c^{-w t \%: ~} 49.3$ \\
\hline Volatiles $_{650-950 c^{-w 1} \%: 8.8}$ \\
\hline Fixed carbon-wt $\%: 7.1$ \\
\hline Ash-wt\%: 34.8 \\
\hline
\end{tabular}

(Pahokee Point, Fla.) 

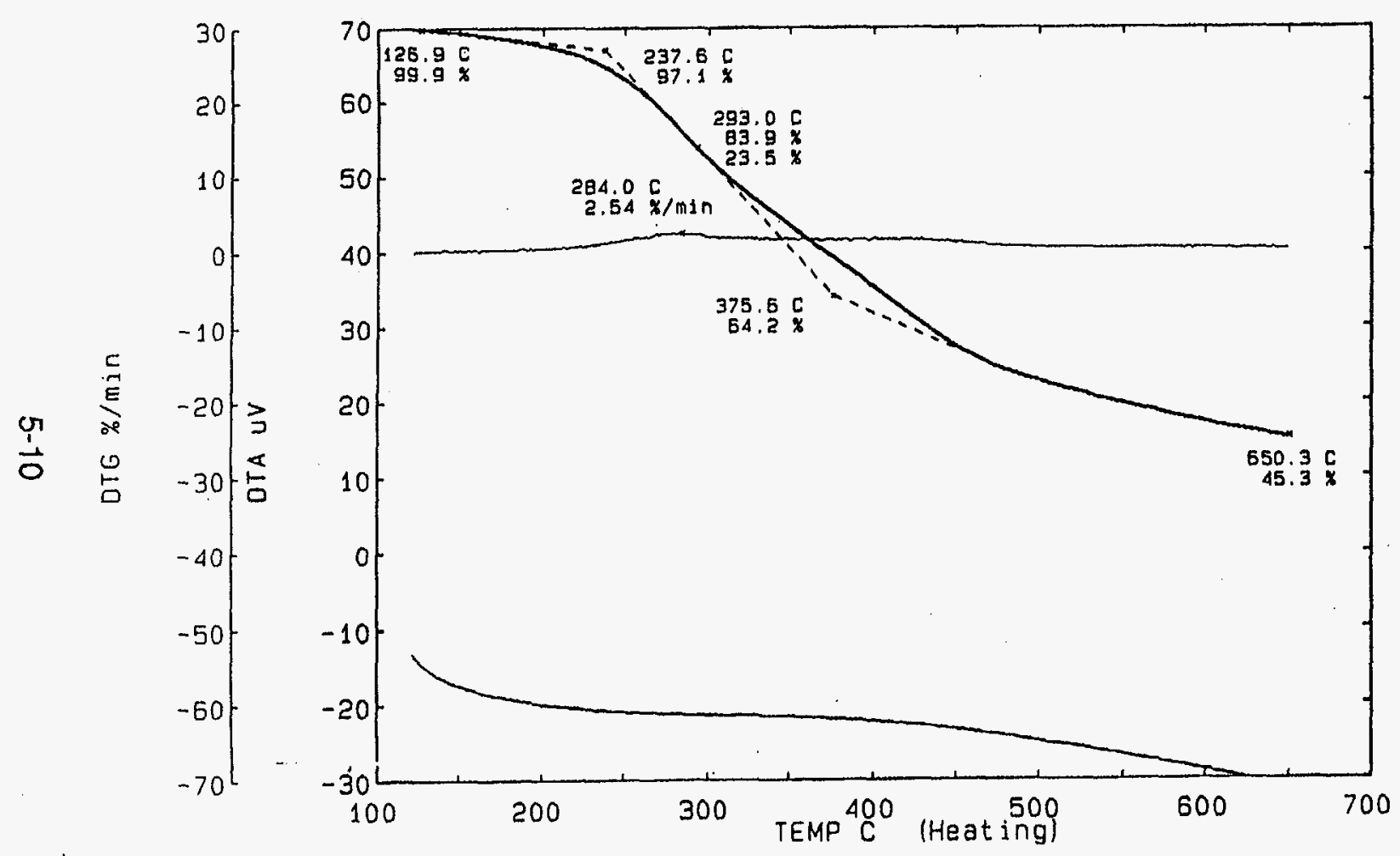

100

90

80

70

60

50

10

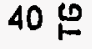

30

20

10

0

Peat

(Steam exploded)

\begin{tabular}{|c|}
\hline $\begin{array}{c}\text { Peat } \\
\text { (Steam exploded) }\end{array}$ \\
\hline Heating rate- ${ }^{\circ} \mathrm{C} / \mathrm{min}: 10$ \\
\hline Samplo weight-mg: 2.117 \\
\hline $\mathrm{T}_{\text {melut }}{ }^{\circ} \mathrm{C}: 126.9$ \\
\hline Volatilization \\
\hline $\mathrm{T}_{\text {oun-polax }}{ }^{\circ} \mathrm{C}: 237.6$ \\
\hline $\mathrm{T}_{\text {mid.poles }}{ }^{-0} \mathrm{C}: 293.0$ \\
\hline$T_{\text {codppolat }}{ }^{\circ} \mathrm{C}: 375.6$ \\
\hline$T_{\max } x^{-0} \mathrm{C}: 284.0$ \\
\hline Rate $_{\max }-\mathrm{mg} / \mathrm{min}: 2.54$ \\
\hline Wt. \% ose c: 45.3 \\
\hline$\Delta \mathrm{H}_{\mathrm{vol},}-\mu \mathrm{v}, \mathrm{sec} / \mathrm{mg}: \mathrm{NS}$ \\
\hline Proximate analysis \\
\hline Volatiles $_{\text {Toun }}$-wt \%: 61.5 \\
\hline Volatiles sso $\mathrm{c}^{-w t \%: ~} 54.7$ \\
\hline Volatiles $_{\Delta 50.950} c^{-w t \%: ~} 6.8$ \\
\hline Fixed carbon-wt $\%: 3.8$ \\
\hline Ash-wt\%: 34.7 \\
\hline
\end{tabular}




\section{Chapter 6}

\section{LIQUID FUELS}

Biomass suffers from being a solid fuel, hard to collect, hard to store, hard to feed into combustion equipment. The simplest liquid fuels made from biomass are methanol (wood alcohol) from synthesis gas; ethanol (grain alcohol) made by fermentation of sugars from starch or cellulose. However, these chemical compounds have exact boiling points, so that TGA is not applicable. However, there is wide interest in a number of other liquid fuels from biomass. A recent survey [Stevens, 1994] summarizes ten years of work of the Department of Energy Laboratories on conversion of biomass, primarily to liquid and gas fuels.

\subsection{Vegetable oil}

Vegetable oils such as olive oil and animal fats such as whale oil and tallow have been used for fuels and for cooking for thousands of years. They are remarkably similar in composition, being the esters of glycerol with high molecular weight fatty acids of primarily 16-18 carbon atoms. Most animal fats contain saturated fatty acids primarily stearic, $\left(\mathrm{C}_{18}\right)$ and are solid at room temperature. Vegetable oil fatty acids typically have one or two double bonds (oleic, linoleic acids) and are liquid at room temperature [Swern, 1979].

The very high vaporization temperature of fats and oils (triglycerides) accounts for their use in cooking. However, it makes them less desirable as lighting or diesel fuels.6.2 Biodiesel

\subsection{Biodiesel}

Recently a superior diesel fuel called biodiesel has been made from the renewable fats and oils by transesterification with methanol or ethanol [Reed, 1992; Reed, 1993]. The resulting fuel has a high Cetane number and low sulfur which leads to clean combustion in diesel engines.

Conversion of the corn oil to its methyl ester lowers the vaporization temperature by $150^{\circ} \mathrm{C}$ which is part of the reason it can be used as an alternate diesel fuel.

\subsection{Pyrolysis Oils}

If the volatile materials emitted during charcoal manufacture (slow pyrolysis) are condensed, they form an aqueous layer, pyroligneous acid and an insoluble layer of tar. Many chemicals can be separated from these materials and they formed the basis of our chemical industries (along with coal volatiles) through the 1930 s.

When biomass is heated rapidly to about $200^{\circ} \mathrm{C}$ it begins to decompose a liquid consisting of the monomer, oligomers and fragments of the polymers hemicellulose, cellulose and lignin. These oils have been called "Fast pyrolysis" oils and they can be used as boiler fuels, for chemical synthesis and even as a diesel fuel. A recent conference explored the use of these oils [NREL, 1994].

Processes are now being developed that give yields up to $70 \%$ (including $10 \%$ water) yields of pyrolysis oils[Scott, 1980; Diebold, 1988; Graham, 1988; NREL, 1994]. A number of laboratories are now producing pyrolysis oils on an experimental basis. Several fast pyrolysis oils are included in this section. 


\subsection{Hydrocarbons}

Crude oil has provided most of the liquid fuel for the 20th century and will continue to provide energy for the next century until it is gone or environmental considerations limit its use. A thermogram of diesel is included in this Atlas for purposes of comparison. A sample of paraffin is also included. 

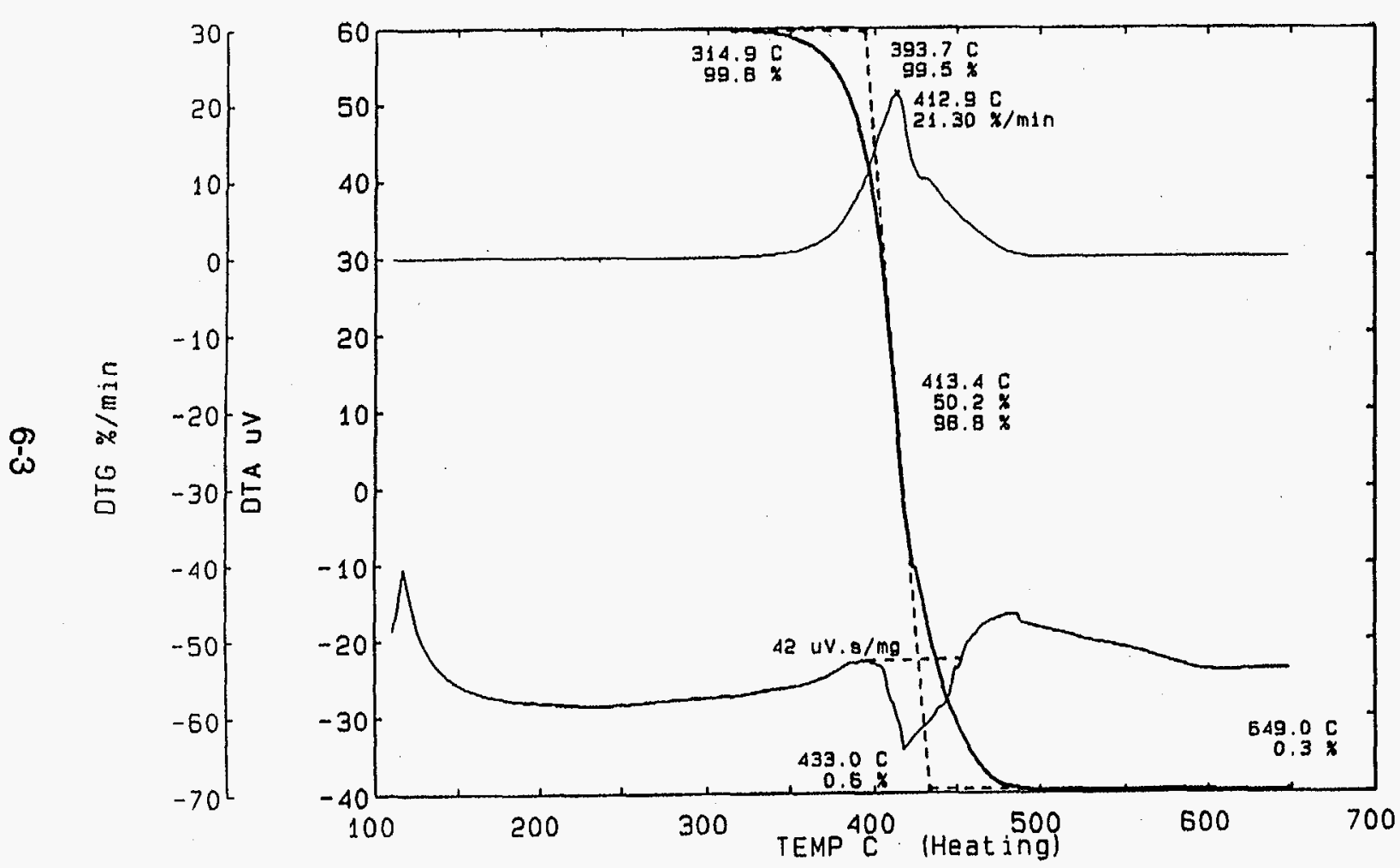

100

80

70

60

50

$\leftrightarrow$

$40 \stackrel{5}{\circ}$

30

20

10

0

Corn oil

(Unsaturated triglyceride)

\begin{tabular}{|c|}
\hline $\begin{array}{c}\text { Corn oil } \\
\text { (Unsaturated triglyceride) }\end{array}$ \\
\hline Heating rate- ${ }^{\circ} \mathrm{C} / \mathrm{min}: 10$ \\
\hline Sample weight-mg: 15.023 \\
\hline $\mathrm{T}_{\text {toltulat }}{ }^{-\mathrm{C}:} \mathbf{3 1 4 . 9}$ \\
\hline Volatilization \\
\hline$T_{\text {ourtpotan: }}{ }^{\circ} \mathrm{C}: 393.7$ \\
\hline$T_{m b-p o l a x} 0^{\circ} \mathrm{C}: 413.4$ \\
\hline$T_{\text {candpolat }}{ }^{\circ} \mathrm{C}: 433.0$ \\
\hline $\mathrm{T}_{\max }{ }^{\circ} \mathrm{C}: \quad 393.7$ \\
\hline Rate $_{\max }=\mathrm{mg} / \min : 21.30$ \\
\hline Wt. \% aso c $c^{-:} 0.3$ \\
\hline$\Delta H_{r o l}-\mu v . s e c / m g: ~ 42$ \\
\hline Proximate analysis \\
\hline Volatiles $_{\text {Tou }^{-}-\mathrm{W}}{ }^{2} \%: 100.0$ \\
\hline Volatiles as $c^{-w t} \%: 989.7$ \\
\hline Volatiles ${ }_{000-500} c^{-w t} \%: 0.3$ \\
\hline Fixed carbon-wt\%: 0.0 \\
\hline Ash-wt\%: 0.0 \\
\hline
\end{tabular}



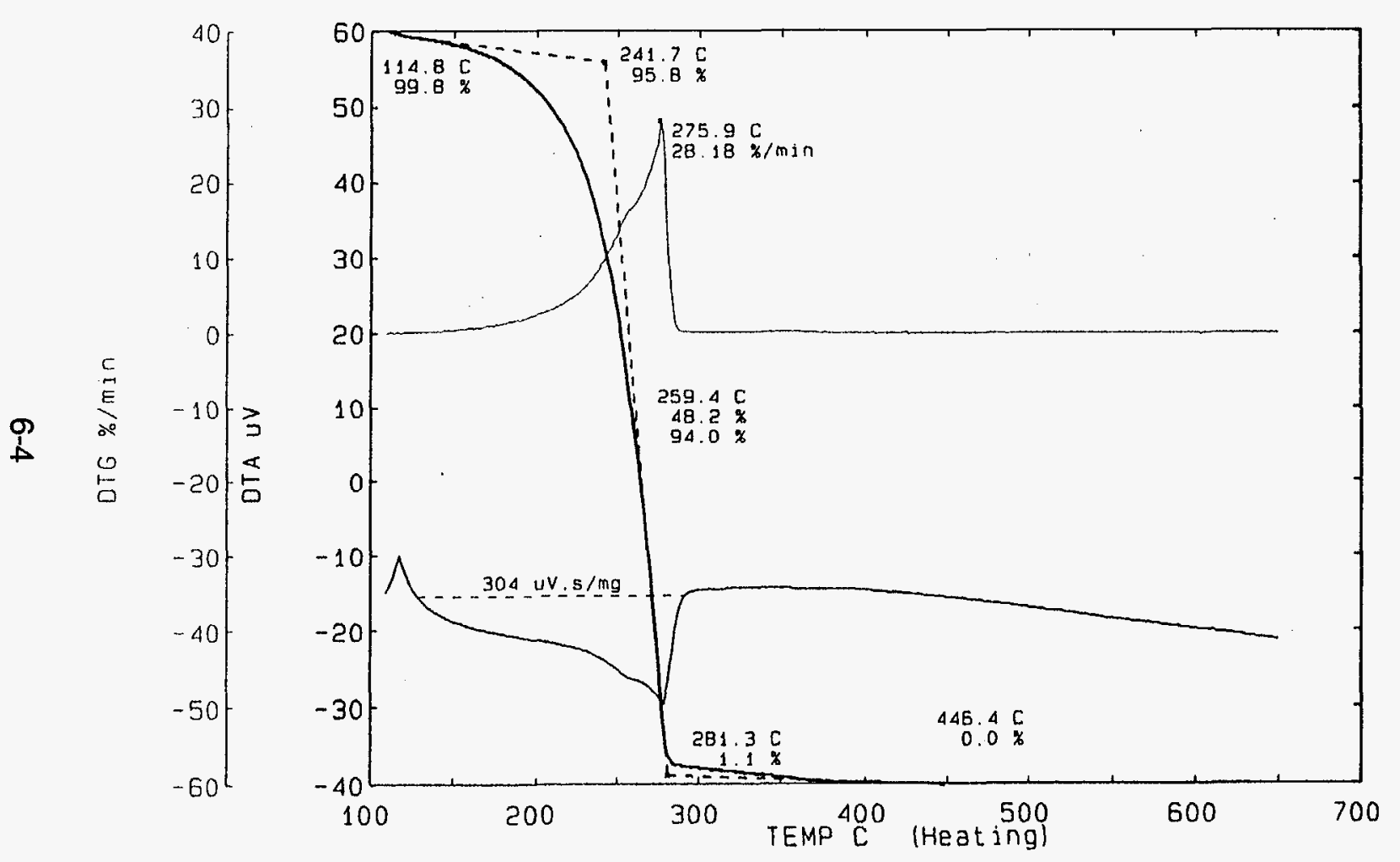

100

90

Bo

70

60

50

se

$40 \%$

30

20

10

Biodiesel

(Corn Oil - methyl ester)

\begin{tabular}{|c|}
\hline $\begin{array}{c}\text { Biodiesel } \\
\text { (Corn Oil - methyl ester) }\end{array}$ \\
\hline Heating rate- ${ }^{\circ} \mathrm{C} / \mathrm{min}: 10$ \\
\hline Sample weight-mg: 19.81 \\
\hline$T_{\text {Lniltal }}{ }^{\circ} \mathrm{C}: 114.8$ \\
\hline Volatilization \\
\hline$T_{\text {olart-potat }}{ }^{\circ} \mathrm{C}: 241.7$ \\
\hline $\mathrm{T}_{\text {midpopint }}{ }^{\circ} \mathrm{C}: \quad 259.4$ \\
\hline$T_{\text {end point }}{ }^{\circ} \mathrm{C}: 281.3$ \\
\hline $\mathrm{T}_{\max }{ }^{-{ }^{\circ} \mathrm{C}: 275.9}$ \\
\hline Rate $_{\max }-\mathrm{mg} / \mathrm{min}: 28.18$ \\
\hline Wt. \% $650 \mathrm{c}^{-:} 0.0$ \\
\hline$\Delta \mathrm{H}_{\mathrm{vol}}, \mu \mathrm{vv} . \mathrm{sec} / \mathrm{mg}: 304$ \\
\hline Proximate analysis \\
\hline Volatiles $_{\text {Tout }}-\mathrm{wt} \%: 100.0$ \\
\hline Volatiles ${ }_{\text {sso }} c^{-w t} \%: 100.0$ \\
\hline Volatiles $_{\text {ssa-9so }} \mathrm{c}^{-w t} \%: 0.0$ \\
\hline Fixed carbon-wt\%: 0.0 \\
\hline Ash-wt \%: 0.0 \\
\hline
\end{tabular}



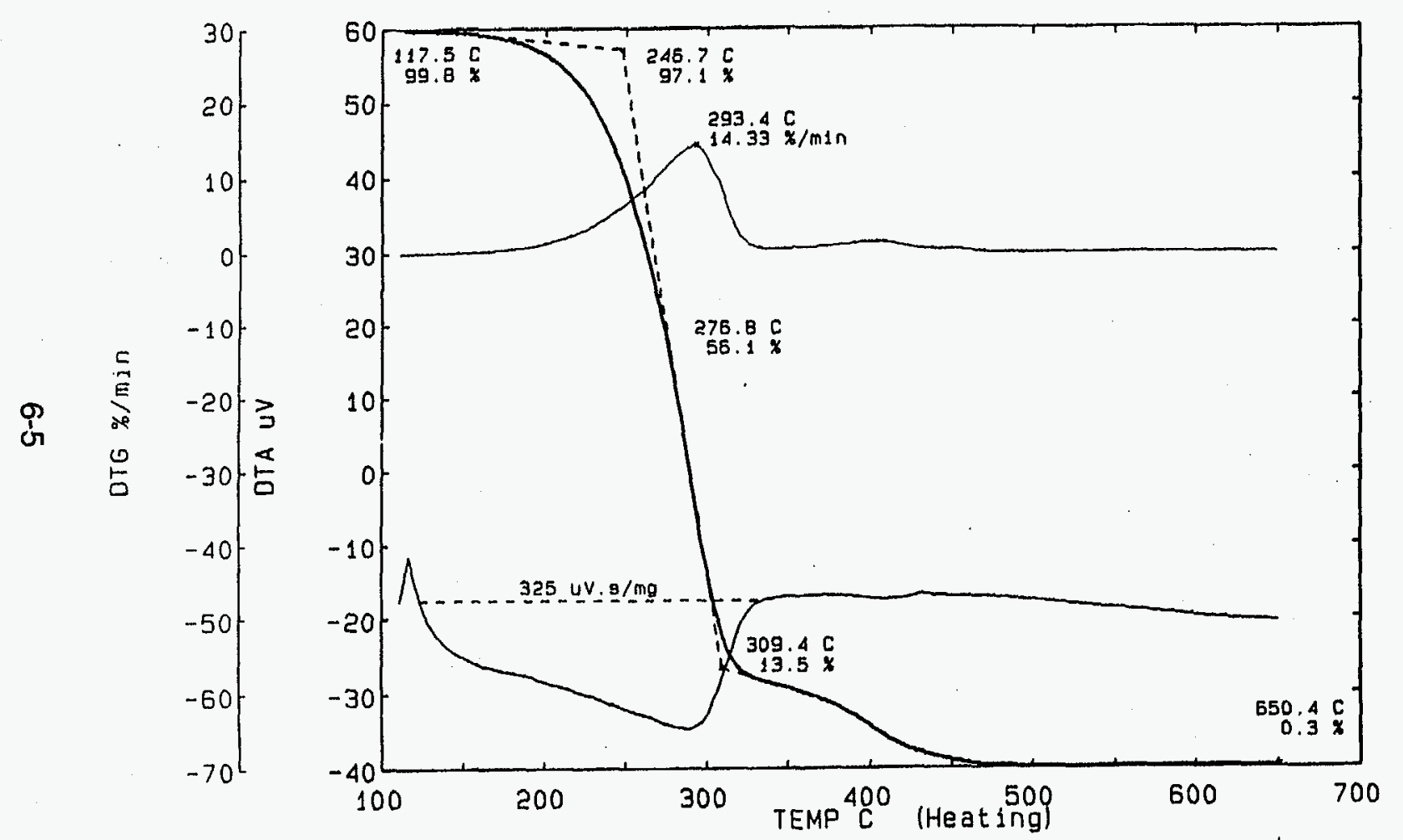

100

90

80

70

60

50

$x$

$40 \%$

30

20

10

$-70$

Waste vegetable oil

(Methyl ester)

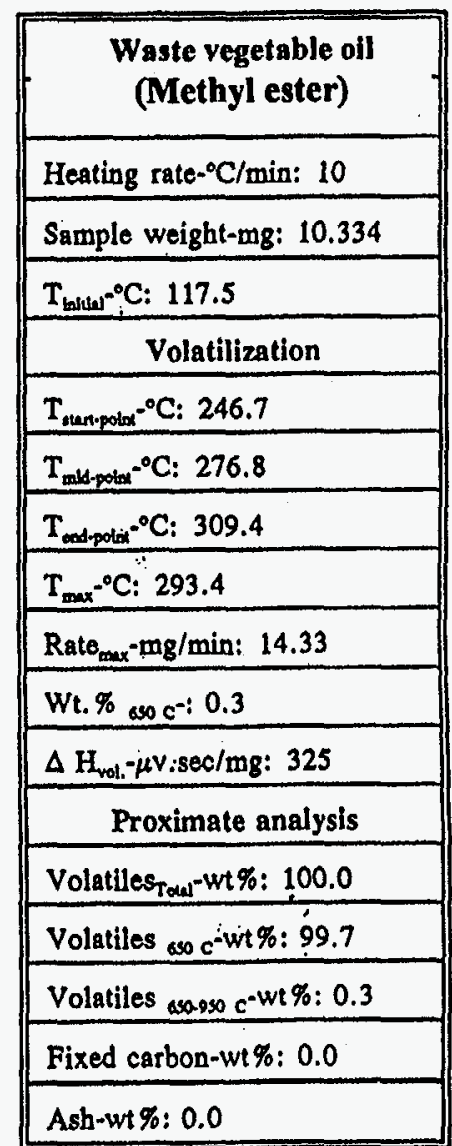




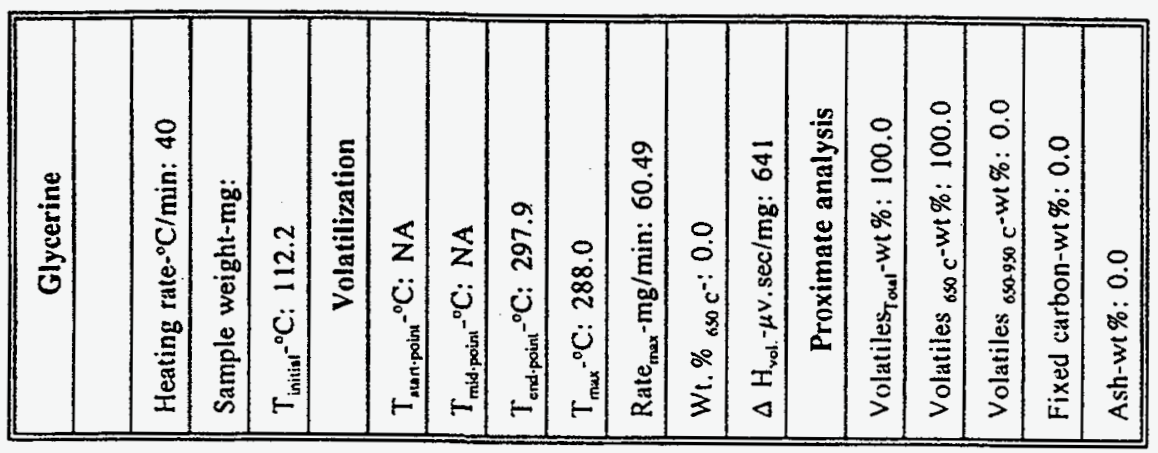

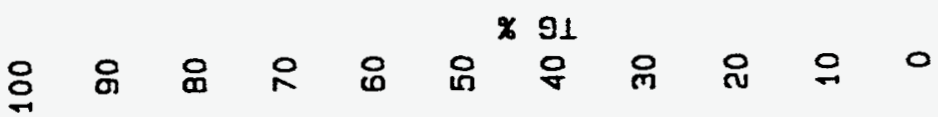

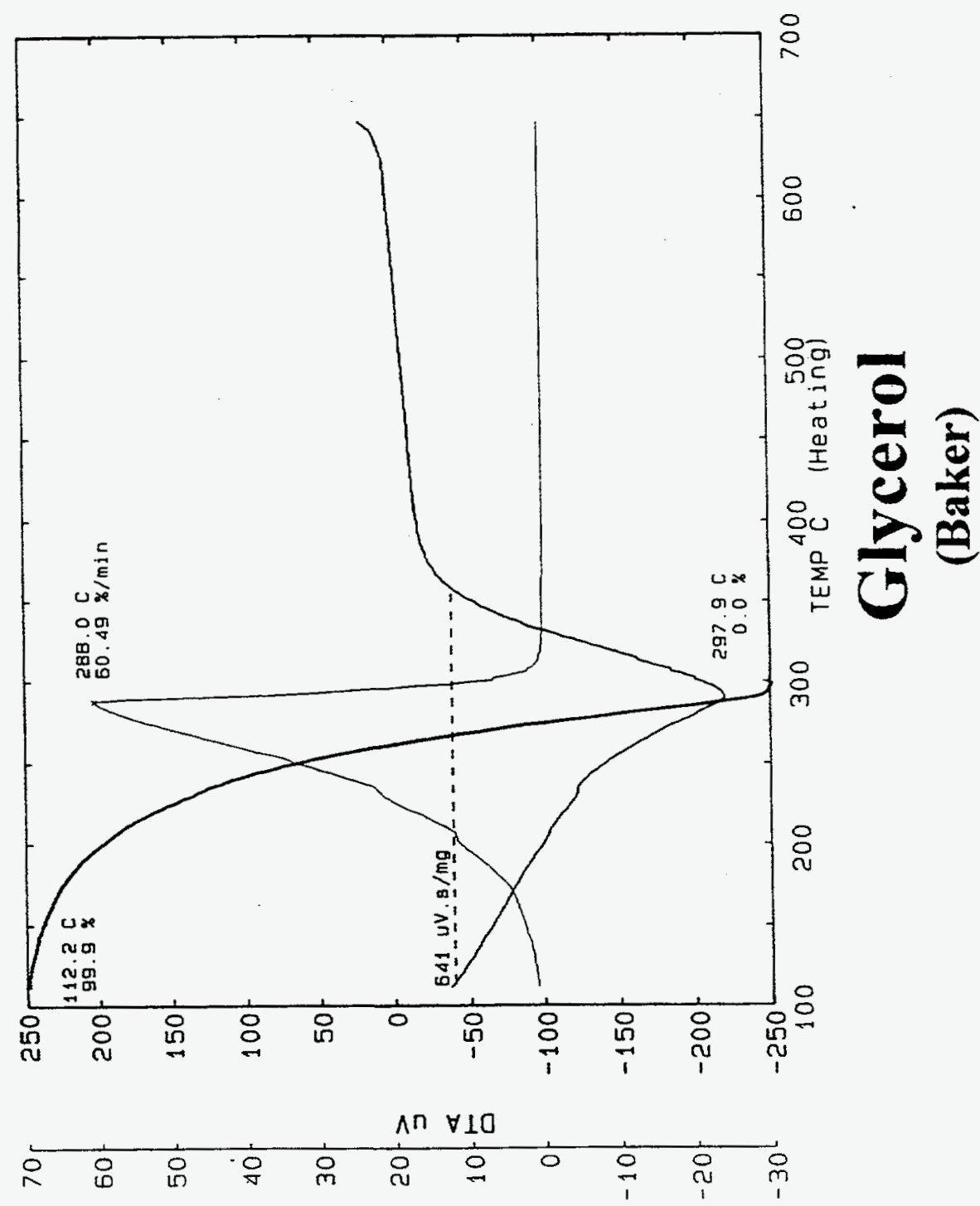

utu/\% 910 


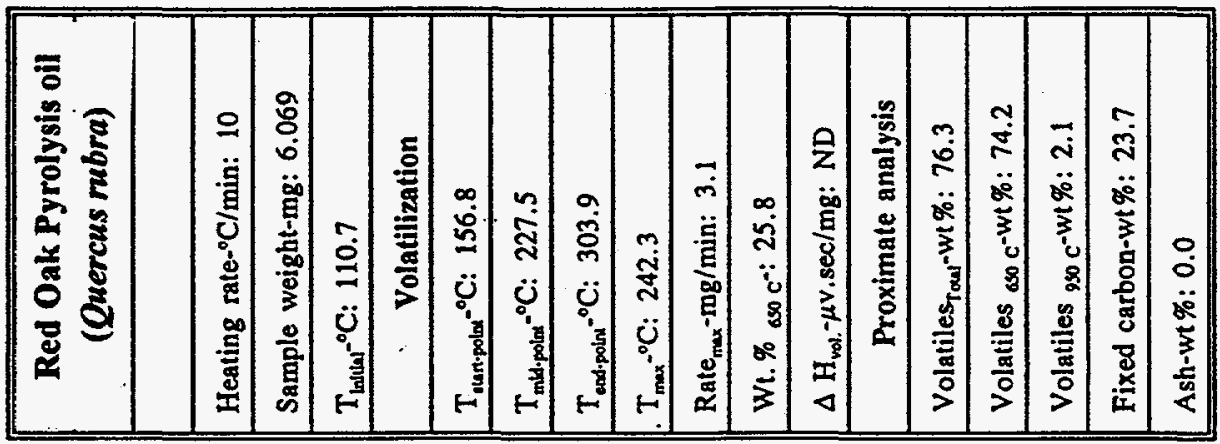

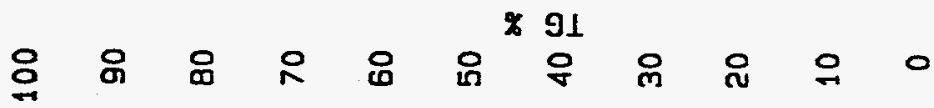

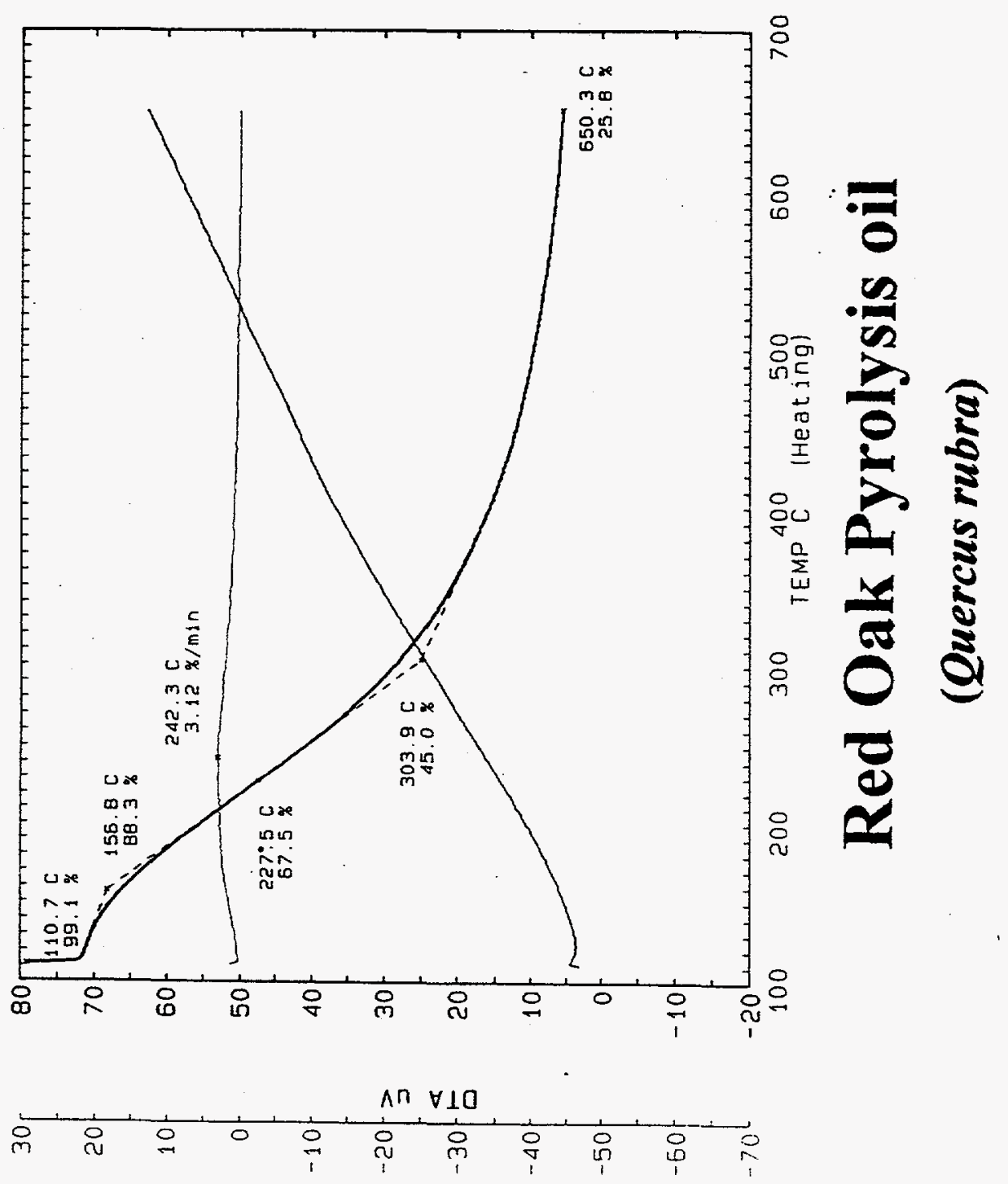

uโய $/ \% 910$ 

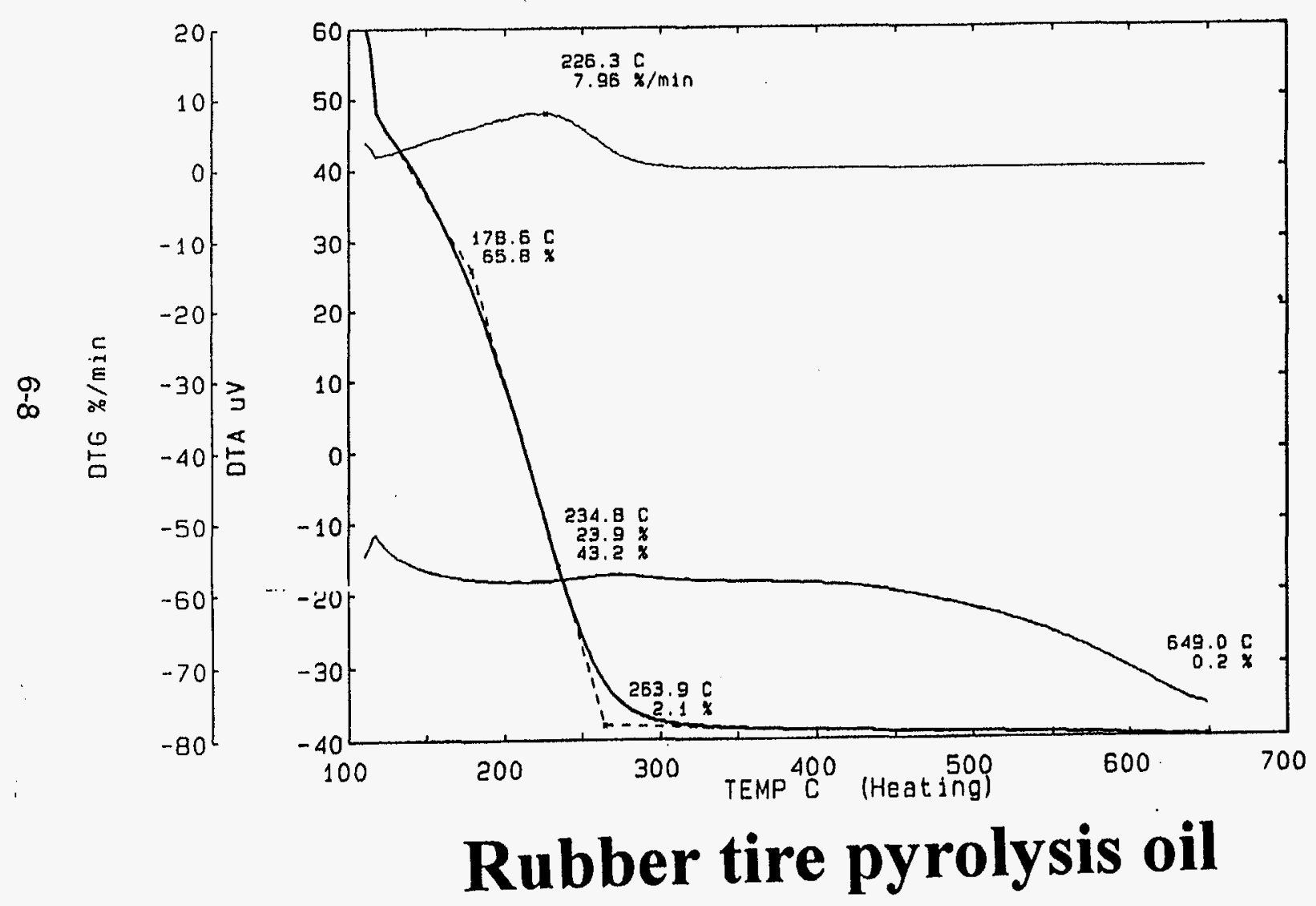

\begin{tabular}{|c|c|}
\hline 90 & $\begin{array}{l}\text { Rubber tire } \\
\text { Pyrolysis oil }\end{array}$ \\
\hline 80 & Heating rate ${ }^{\circ} \mathrm{C} / \min : 10$ \\
\hline 70 & Sample weight-mg: 7.382 \\
\hline \multirow{2}{*}{60} & $\left.T_{\text {londul| }}\right|^{-0} \mathrm{C}: \mathrm{NA}$ \\
\hline & Volatilization \\
\hline \multirow{3}{*}{$\begin{array}{l}50 \\
40 \stackrel{2}{\circ}\end{array}$} & $T_{\text {suanpolax }}{ }^{-\mathrm{C}}: 178.6$ \\
\hline & $T_{\text {mbt-pobx }}-^{\circ} \mathrm{C}: 234.8$ \\
\hline & $\mathrm{T}_{\text {end-polat }} 0^{\circ} \mathrm{C}: 263.9$ \\
\hline 30 & $\mathrm{~T}_{\max }{ }^{\circ} \mathrm{C}: 226.3$ \\
\hline \multirow{2}{*}{20} & Rate $_{\text {max }}-\mathrm{mg} / \mathrm{min}: 7.96$ \\
\hline & Wt. $\%_{0 s 0} c^{-:}: 0.2$ \\
\hline \multirow[t]{2}{*}{10} & $\Delta \mathrm{H}_{\mathrm{rol}}-\mu \mathrm{v} . \mathrm{sec} / \mathrm{mg}: \mathrm{NA}$ \\
\hline & Proximate analysis \\
\hline \multirow[t]{5}{*}{0} & 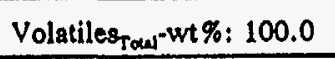 \\
\hline & Volatiles $_{s s 0} c^{-w t} \%: 99.8$ \\
\hline & Volatiles $_{\text {ssa-9so }} c^{-w t} \%: 0.2$ \\
\hline & Fixed carbon-wt\%: 0.0 \\
\hline & Ash-wt\%: 0.0 \\
\hline
\end{tabular}




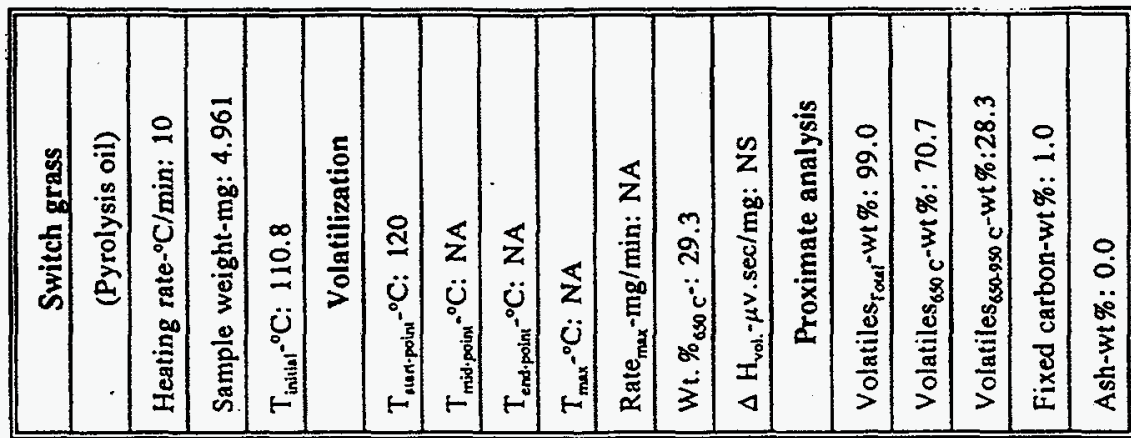

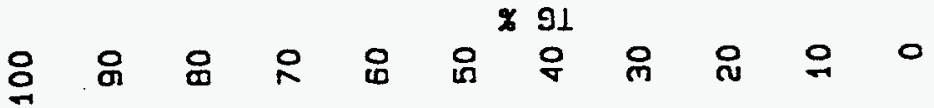
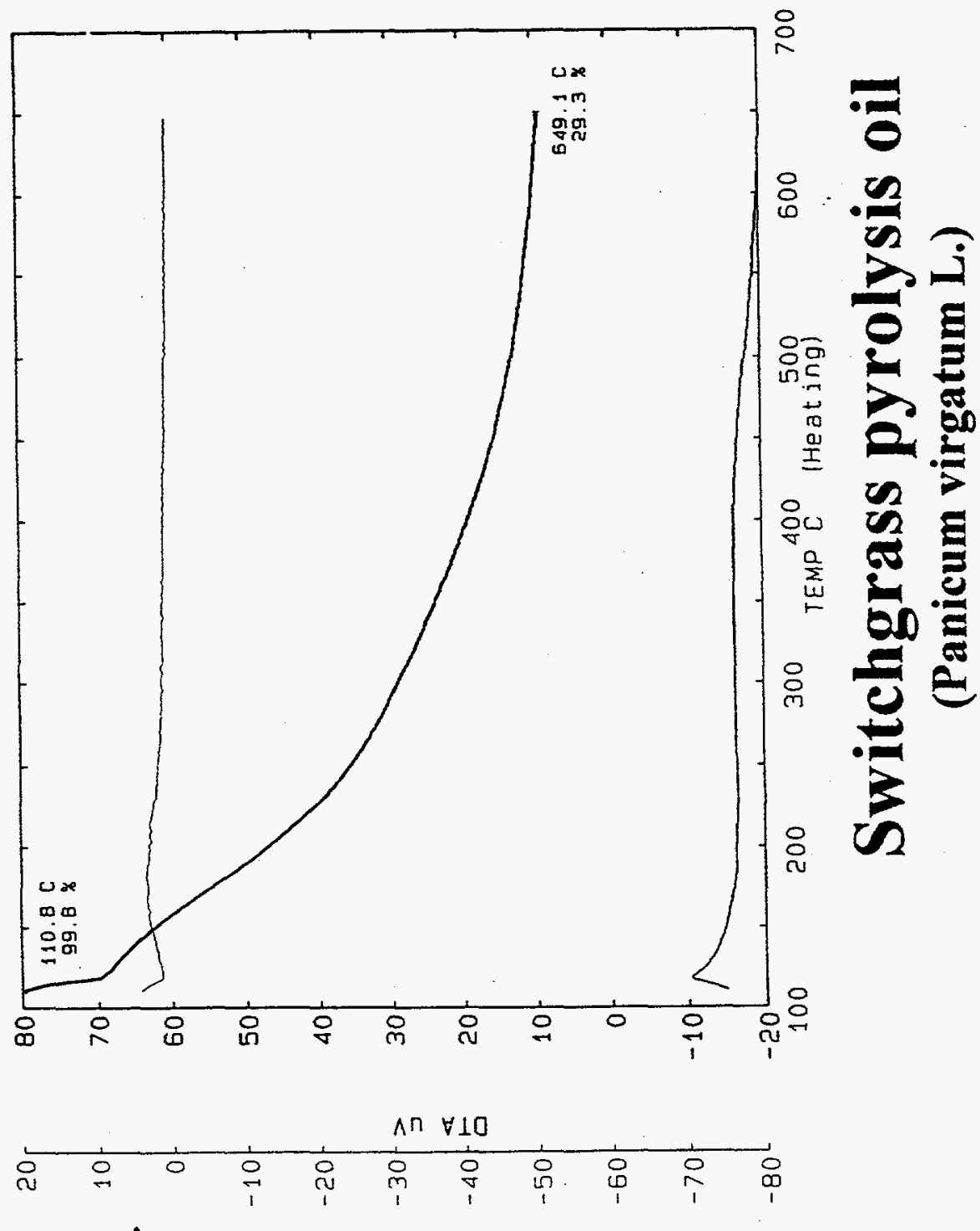

uโฺแ/\% 910 

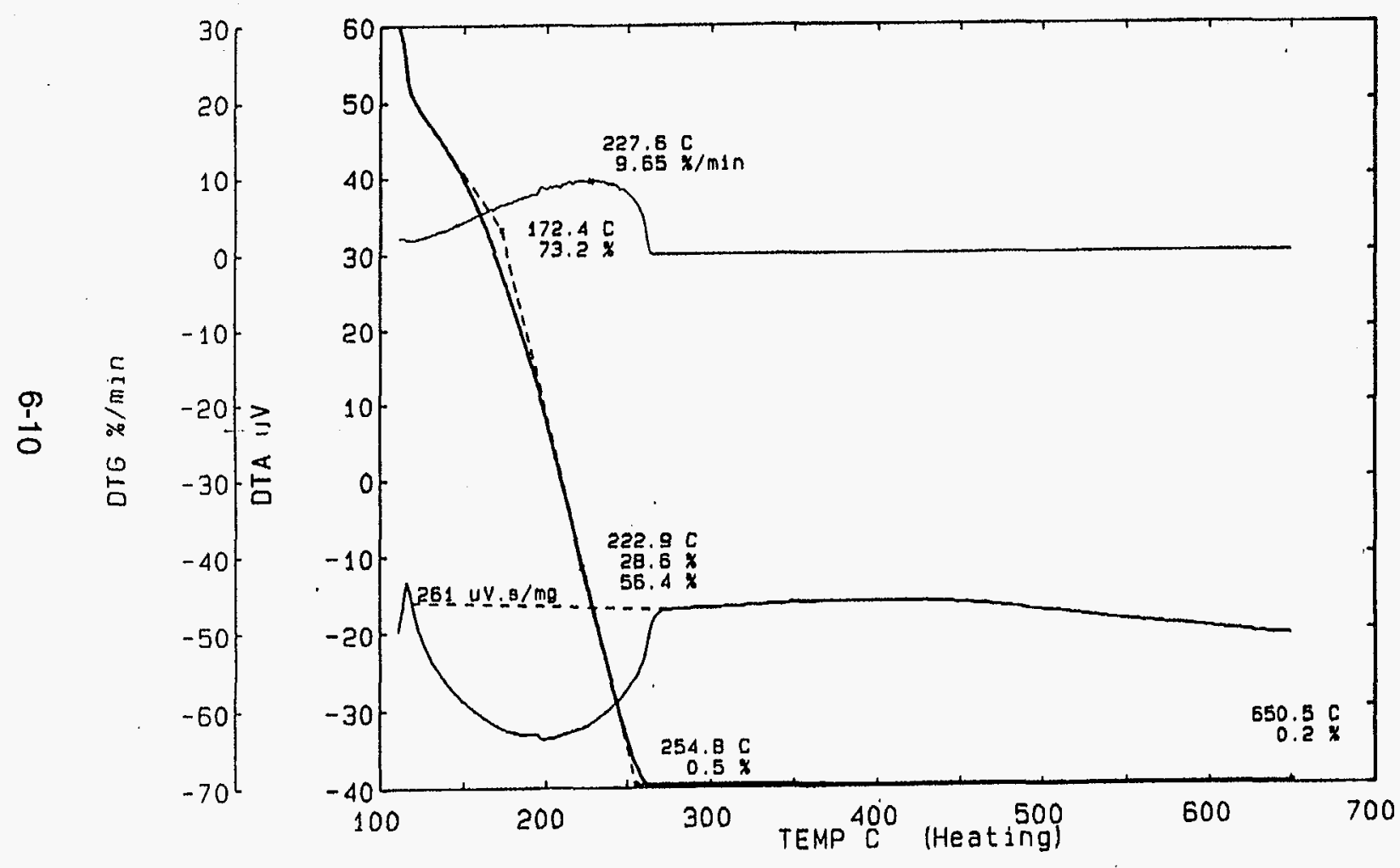

\begin{tabular}{|c|c|}
\hline & Diesel fuel \\
\hline \multicolumn{2}{|l|}{90} \\
\hline \multirow{2}{*}{ BO } & Heating rate- ${ }^{\circ} \mathrm{C} / \mathrm{min}: 10$ \\
\hline & Sample weight-mg: \\
\hline 70 & $\mathrm{~T}_{\text {Inluat }}{ }^{\circ} \mathrm{C}:<110$ \\
\hline \multirow{2}{*}{60} & Volatilization \\
\hline & $T_{\text {tun poial }}{ }^{\circ} \mathrm{C}: 172.4$ \\
\hline \multirow{3}{*}{ 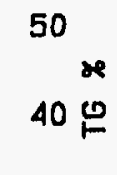 } & $T_{\text {matpolat }}{ }^{\circ} \mathrm{C}: 222.9$ \\
\hline & $T_{\text {exd-polat }}{ }^{\circ} \mathrm{C}: 254.8$ \\
\hline & $T_{\operatorname{mex}}{ }^{\circ} \mathrm{C}: 227.6$ \\
\hline 30 & Rate $_{\max }-\mathrm{mg} / \min : 9.65$ \\
\hline \multirow{2}{*}{20} & Wt. $\%$ asoc $c^{-1} 0.5$ \\
\hline & $\Delta \mathrm{H}_{\mathrm{rel},}, \mu \mathrm{v} . \mathrm{sec} / \mathrm{mg}: 261$ \\
\hline \multirow[t]{2}{*}{10} & Proximate analysis \\
\hline & Volatiles $_{\text {Tow }}-w t \%: 100.0$ \\
\hline \multirow[t]{4}{*}{0} & Volatiles ${ }_{a s 0} c^{-w t} \%: 99.8$ \\
\hline & Volatiles $_{\text {ssa-0so }} c^{-w t} \%: 0.2$ \\
\hline & Fixed carbon-wt\%: 0.0 \\
\hline & Ash-wt \%: 0.0 \\
\hline
\end{tabular}

Diesel fuel 


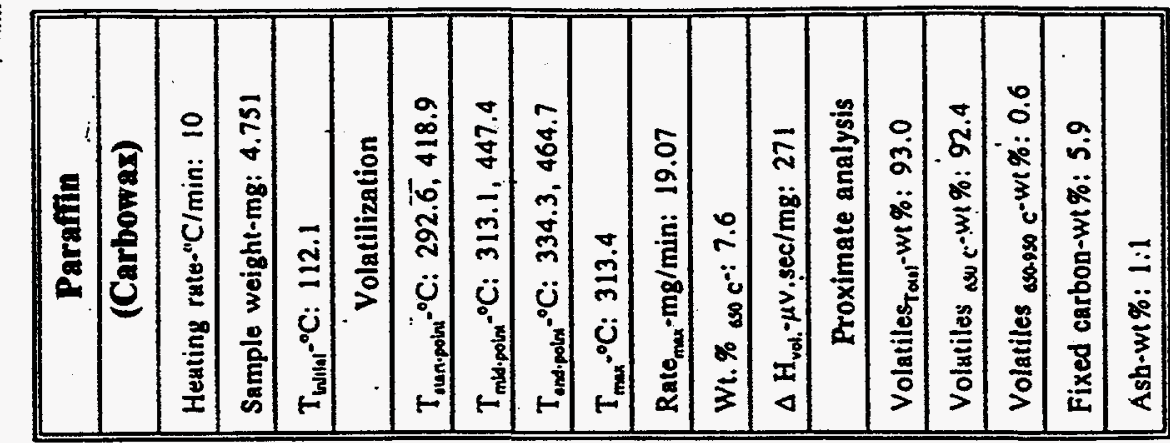

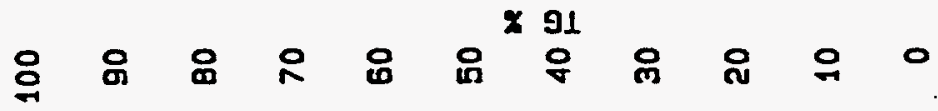
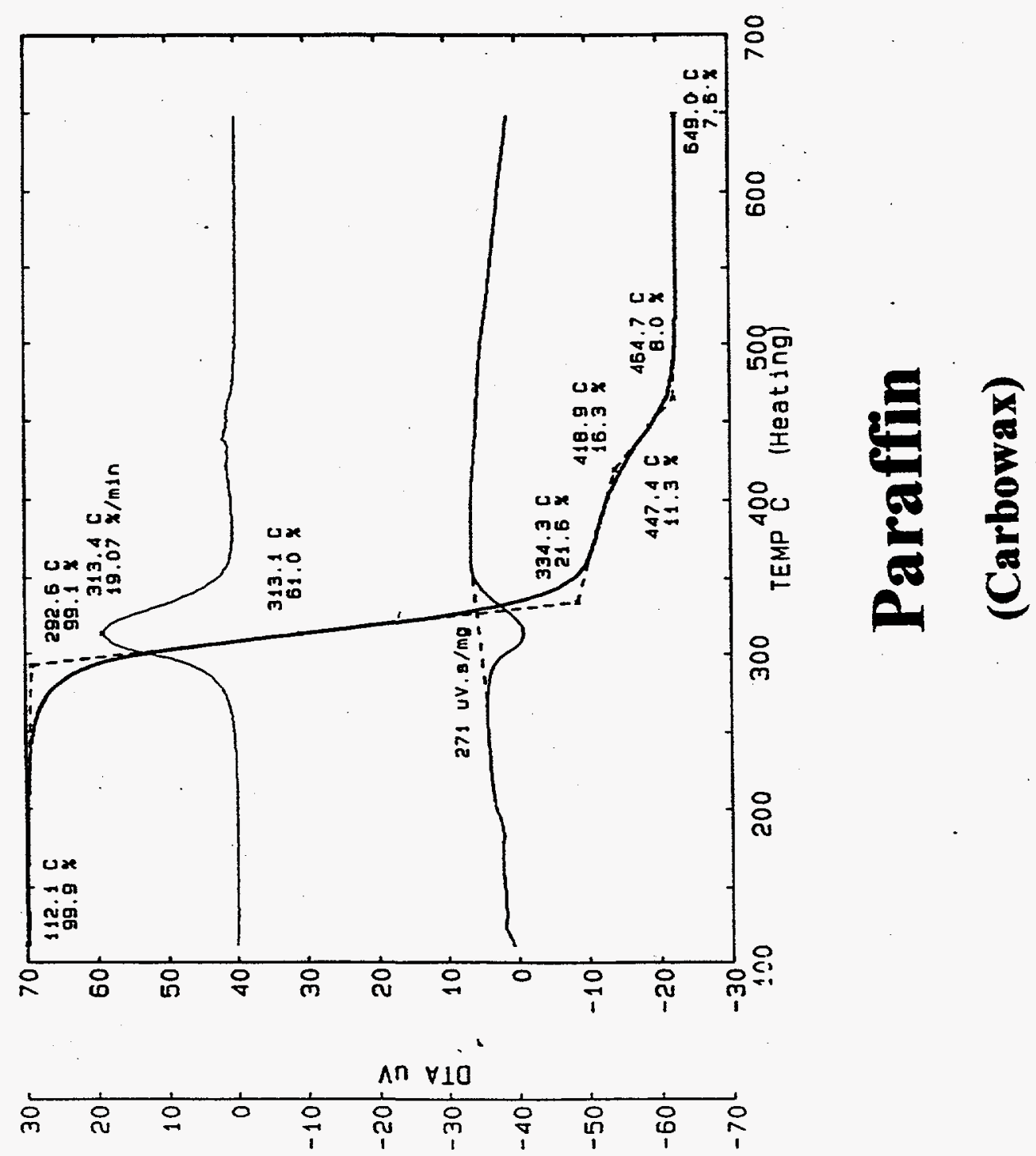

Uțแ/\% 910 


\section{Chapter 7}

\section{VARIATIONS IN BIOMASS}

\subsection{Effect Of Plant Storage}

A number of species supplied by Agblevor at NREL were tested for the effect of storage. Fresh samples were divided into two parts. One part was refrigerated, the other was stored at room temperature for half a year to see the effect of storage.

Examination of the samples shows that storage increased the volatility and decreased the fixed carbon (except for bagasse).

When wood is decomposed in the forest, typically the cellulose and hemicellulose are decomposed, leaving a form of lignin thought to be relatively unaltered. The samples of brown and white rotted woods look very similar to the other lignins, but have a much higher ash content.

\subsection{Effect Of Plant Anatomy}

The various parts of plants have different compositions appropriate to their structures and functions. We show several examples of these differences. We also show several extracts from plants.

\subsection{Effect Of Growth Conditions}

The conditions of growth of a plant affects its composition in terms of volatiles, ash content and fixed carbon. We show the affect of irrigation on the thermal behavior of cotton. 


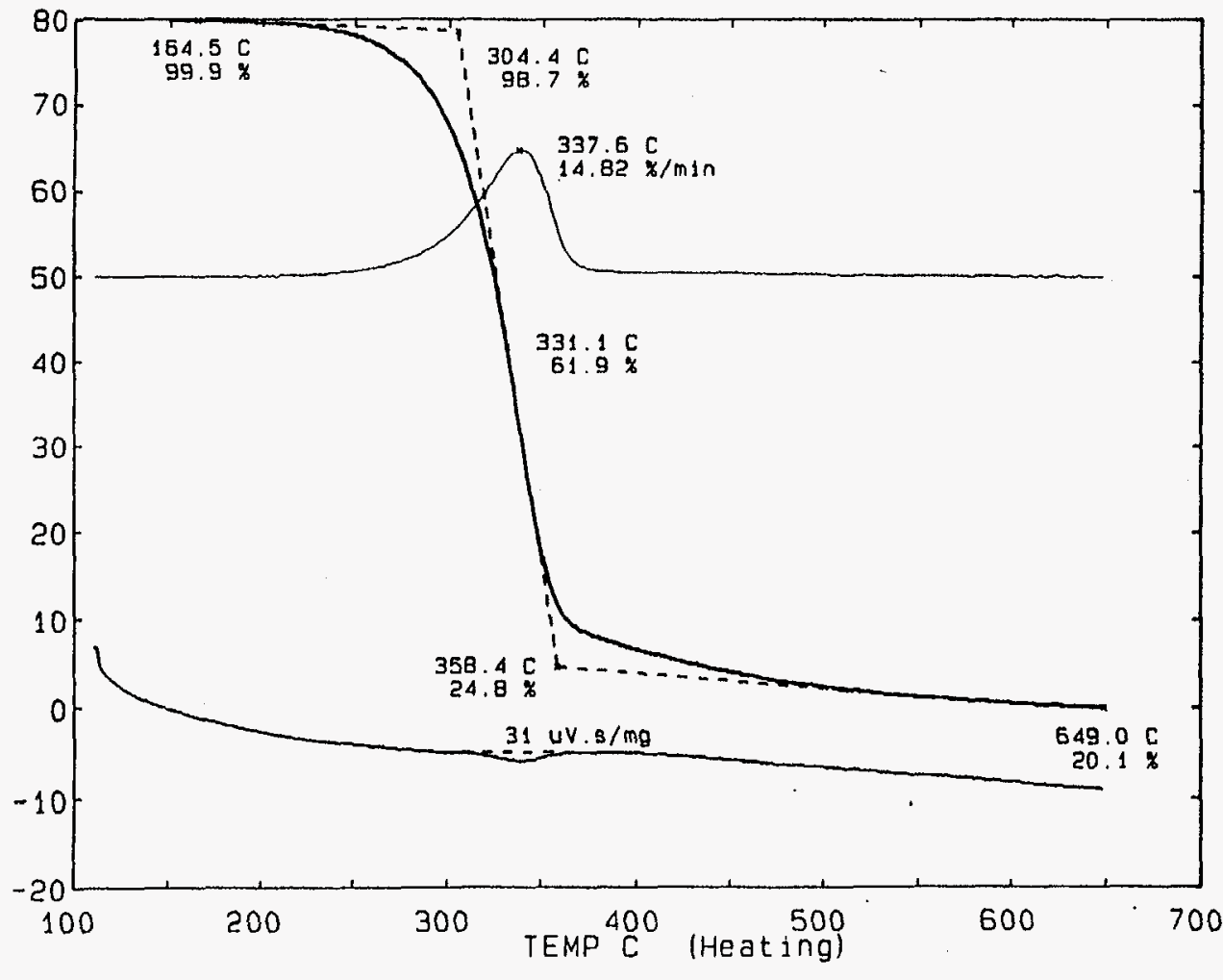

100
90
80
70
60
50
$40 \%$
30
20
10
0

\begin{tabular}{|c|}
\hline $\begin{array}{c}\text { Cotton, dryland } \\
\text { (Gossypium) }\end{array}$ \\
\hline Heating rate- ${ }^{\circ} \mathrm{C} / \mathrm{min}: 10$ \\
\hline Sample weight-mg: 4.884 \\
\hline $\mathrm{T}_{\text {(ndutut }}{ }^{-0} \mathrm{C}: 164.5$ \\
\hline Volatilization \\
\hline 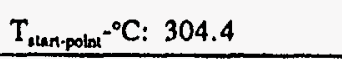 \\
\hline$T_{\text {mb-polum }}{ }^{\circ} \mathrm{C}: 331.1$ \\
\hline$T_{\text {end.poloxt }}{ }^{\circ} \mathrm{C}: \quad 358.4$ \\
\hline$T_{\max }{ }^{\circ} \mathrm{C}: 337.6$ \\
\hline Rate $_{\max }-\mathrm{mg} / \mathrm{min}: 14.82$ \\
\hline Wt. \% csoc c: 20.1 \\
\hline$\Delta \mathrm{H}_{\mathrm{vol}} \cdot \mu \mathrm{v}, \mathrm{sec} / \mathrm{mg}: 31$ \\
\hline Proximate analysis \\
\hline Volatiles $_{\text {Toul }}-w 1 \%: 82.4$ \\
\hline Volatiles sso c$^{-w 1}$ t : $^{\prime} 79.9$ \\
\hline Volatiles ssa.9so $c^{-w 1 \%: ~} 2.5$ \\
\hline Fixed carbon-wt\%: 15.8 \\
\hline Ash-wt\%: 1.8 \\
\hline
\end{tabular}



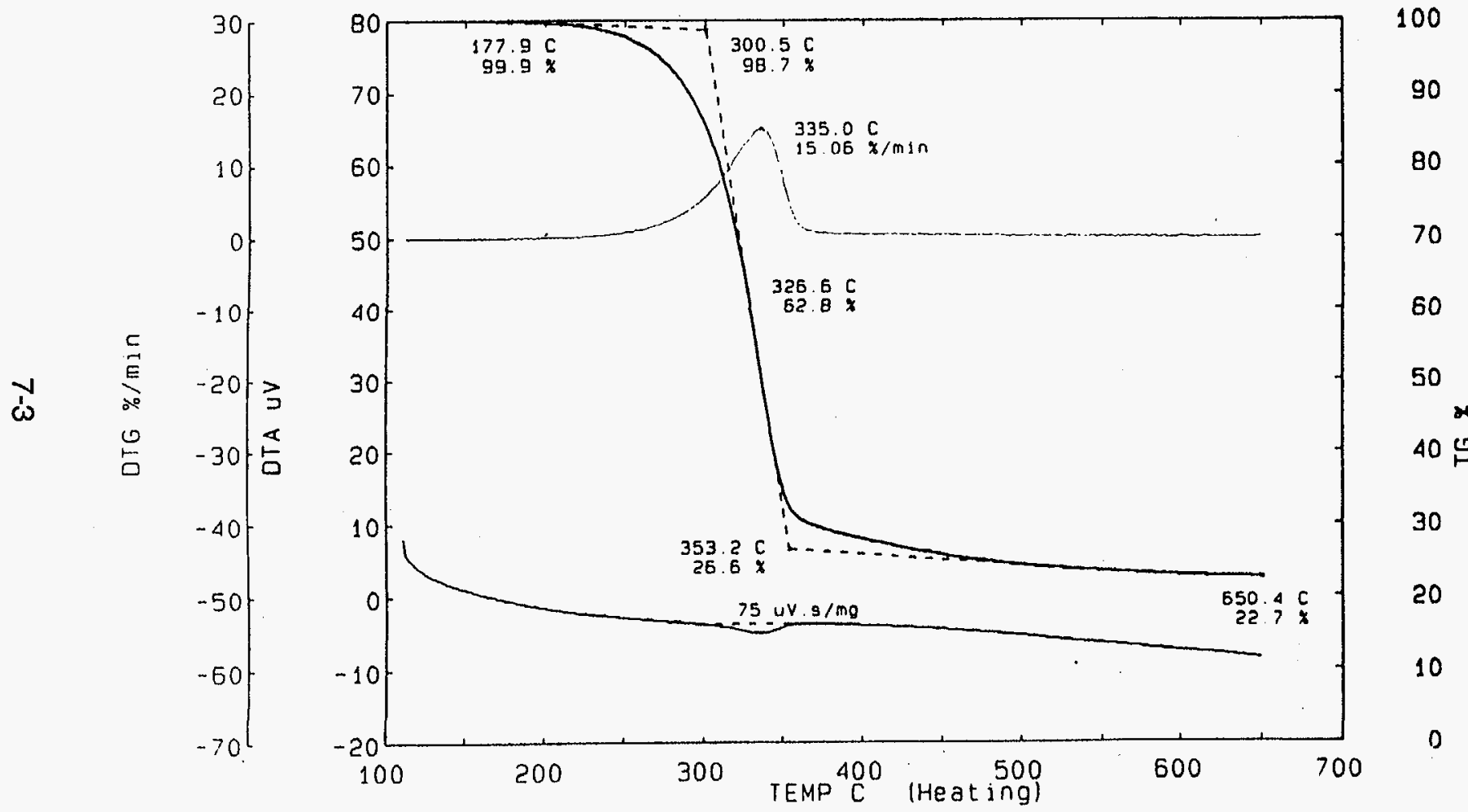

\section{Cotton, irrigated}

(Gossypium)

\begin{tabular}{|c|}
\hline $\begin{array}{l}\text { Cotton, irrigated } \\
\text { (Gossypium }\end{array}$ \\
\hline Heating rate- ${ }^{\circ} \mathrm{C} / \mathrm{min}: 10$ \\
\hline Sample weight-mg: 3.090 \\
\hline$T_{\text {imial }}{ }^{\circ} \mathrm{C}: 177.9$ \\
\hline Volatilization \\
\hline$T_{\text {oun.poinx }}{ }^{\circ} \mathrm{C}: 300.5$ \\
\hline$T_{\text {mis.point }}{ }^{\circ} \mathrm{C}: 326.6$ \\
\hline$T_{\text {end-poins }}{ }^{\circ} \mathrm{C}: 353.2$ \\
\hline$T_{\max }-{ }^{\circ} \mathrm{C}: 335.0$ \\
\hline Rate $_{\operatorname{mu}} \cdot \mathrm{mg} / \mathrm{min}: 15.06$ \\
\hline$W_{1} . \%$ aso c $c^{-:} 22.7$ \\
\hline$\Delta H_{\text {vol }} \cdot \mu \mathrm{v} \cdot \mathrm{sec} / \mathrm{mg}: 75$ \\
\hline Proximate analysis \\
\hline Volatiles $_{\text {Tout }}-w t \%: 79.4$ \\
\hline Volatiles oso $c^{-w 1} \%: 77.3$ \\
\hline Volatiles sso.9so $c^{-w 1 \%:} 2.1$ \\
\hline Fixed carbon-w1\%: 13.4 \\
\hline Ash-w1\%: 7.2 \\
\hline
\end{tabular}



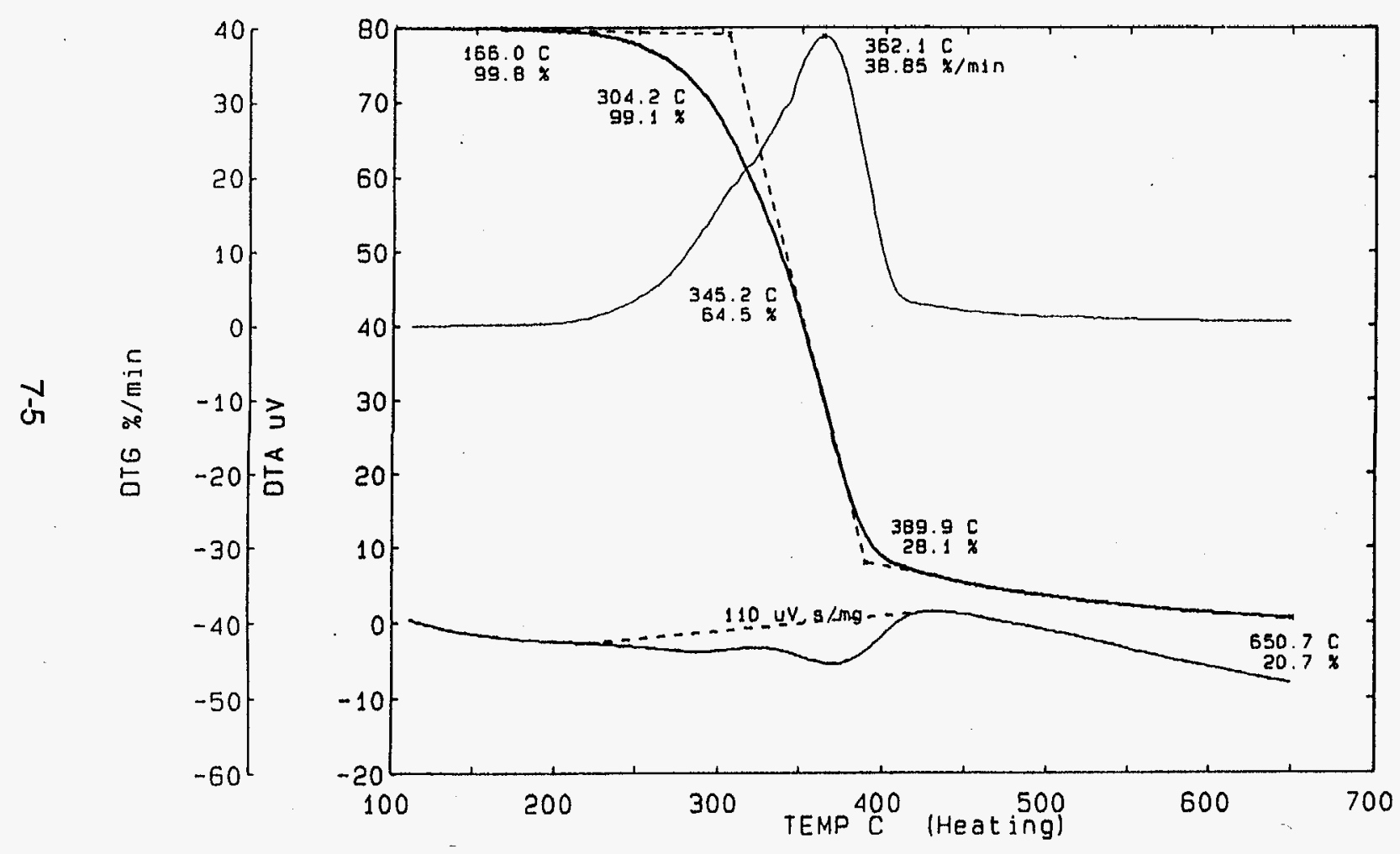

100

90

Eastern Cottonwood Stored Zero Weeks

80

Heating rate- ${ }^{\circ} \mathrm{C} / \mathrm{min}: 40$

Sample weight-mg: 6.390

70

60

50

*e

$40 \stackrel{5}{1}$

30

20

$T_{\text {willat }}{ }^{\circ} \mathrm{C}: 166.0$

\begin{tabular}{||c||}
\hline Volatilization \\
\hline$T_{\text {ounnposin }}{ }^{-0} \mathrm{C}: 304.2$ \\
\hline$T_{\text {mat. polm }}{ }^{-0} \mathrm{C}: 345.2$ \\
\hline
\end{tabular}

Eastern Cottonwood Stored Zero Weeks (Populus deltoides) 


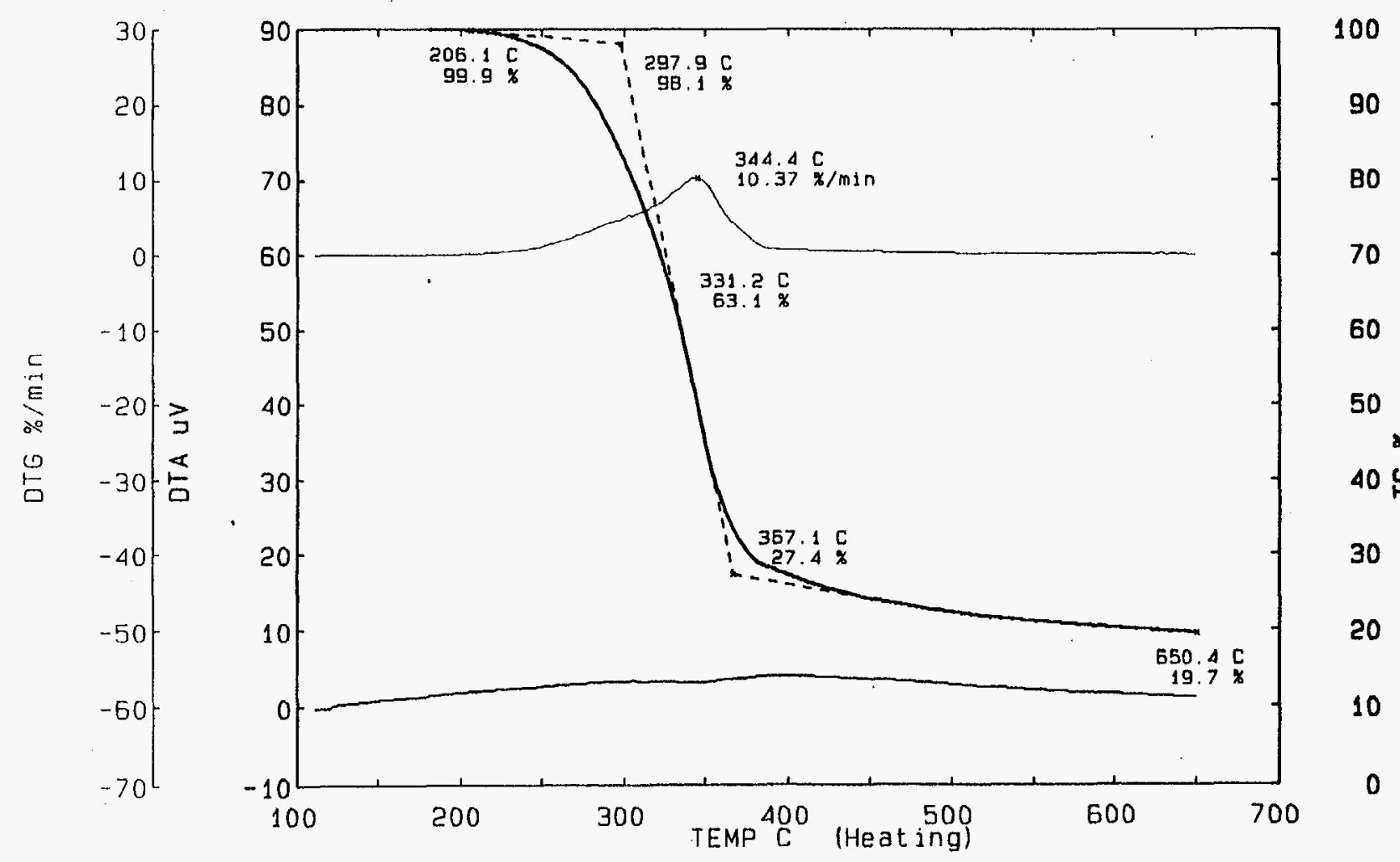

Eastern Cottonwood, Stored 26 weeks

(Populus deltoides) 

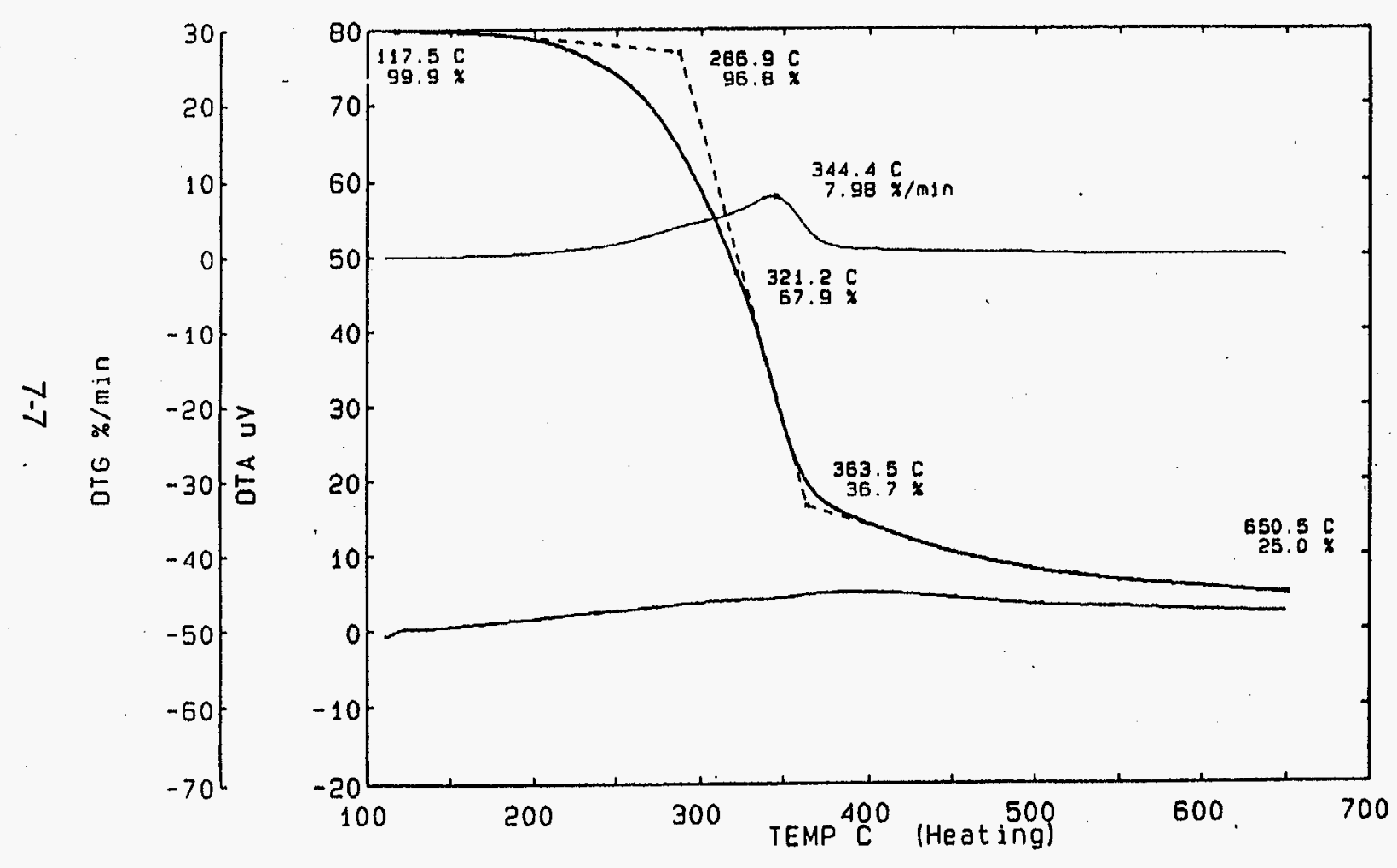

\section{Sericea lespedeza stored for zero weeks}

(Lespedeza cuneata var. serala) 

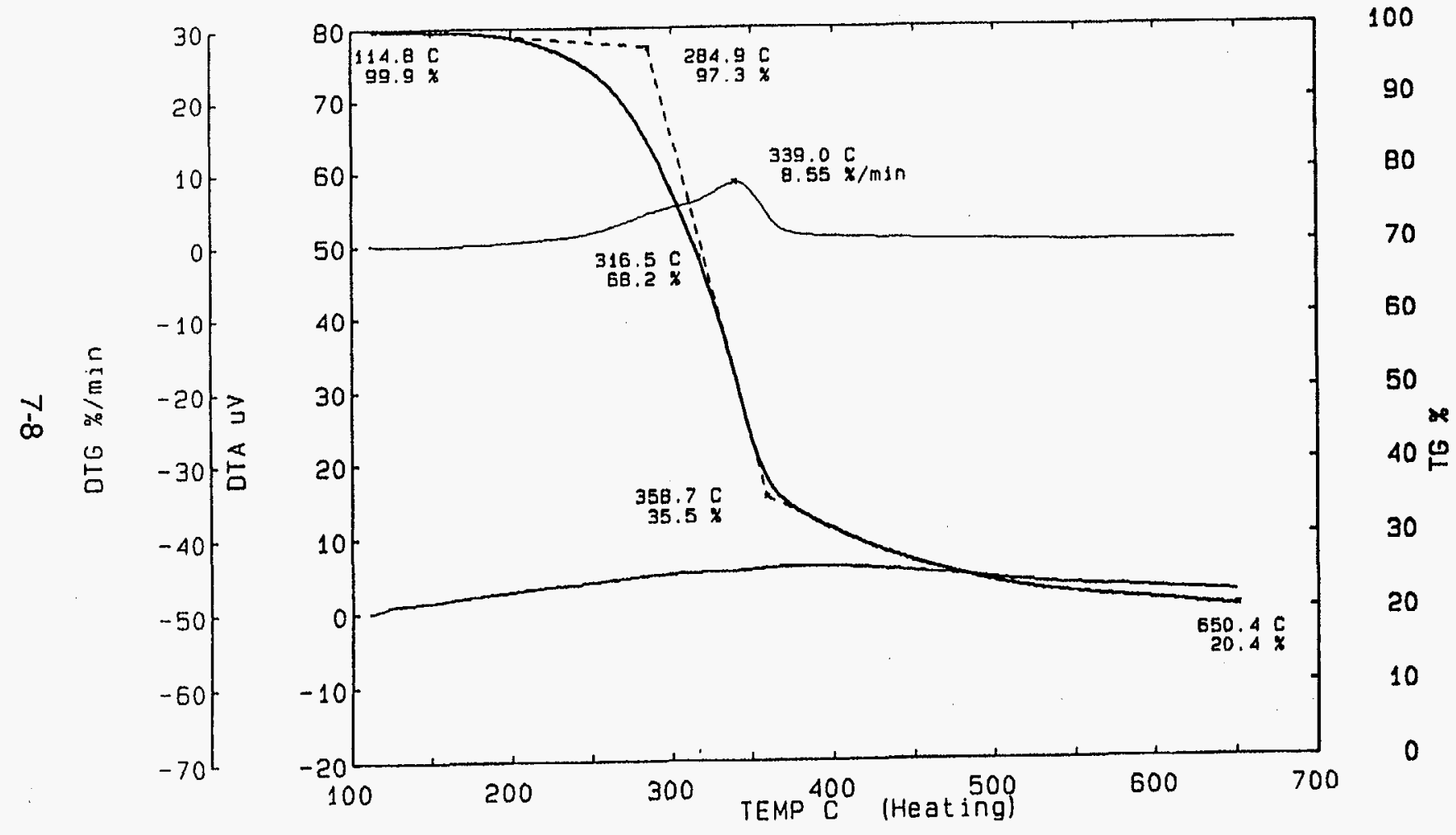

Sericea lespedeza stored for 26 weeks

(Lespedeza cuneata var. serala)

\begin{tabular}{|c|}
\hline $\begin{array}{l}\text { leza stored for } 26 \\
\text { veeks } \\
\text { ineata ver. serala) }\end{array}$ \\
\hline Heating rate $-{ }^{\circ} \mathrm{C} / \mathrm{min}: 10$ \\
\hline Sample weight-mg: 4.391 \\
\hline $\mathrm{T}_{\text {bolutur }} 0^{\circ} \mathrm{C}: 114.8$ \\
\hline Volatilization \\
\hline$T_{\text {olantpolax }}{ }^{\circ} \mathrm{C}: 284.9$ \\
\hline$T_{\text {mid.polax }}{ }^{\circ} \mathrm{C}: 316.5$ \\
\hline $\mathrm{T}_{\text {exd.poles }}{ }^{\circ} \mathrm{C} \mathrm{C}: \quad 358.7$ \\
\hline$T_{\max } x^{\circ} \mathrm{C}: 339.0$ \\
\hline $\mathrm{Rato}_{\max }-\mathrm{mg} / \mathrm{min}: 8.5$ \\
\hline Wl. $\%_{0 s 0} c^{-:} 20.4$ \\
\hline$\Delta \mathrm{H}_{\text {val }}-\mu \mathrm{v} . \mathrm{sec} / \mathrm{mg}: \mathrm{NS}$ \\
\hline Proximate analysis \\
\hline Volatiles $_{\text {Toul }}-w 1 \%: 81.2$ \\
\hline Volatiles $_{\Delta 0_{0} c^{-w 1} \%: 79.6}$ \\
\hline Volatiles $_{\text {ssa-sso }} c^{-w t} \%: 1.6$ \\
\hline Fixed carbon-wt\%: 15.8 \\
\hline Ash-wt\%: 3.0 \\
\hline
\end{tabular}



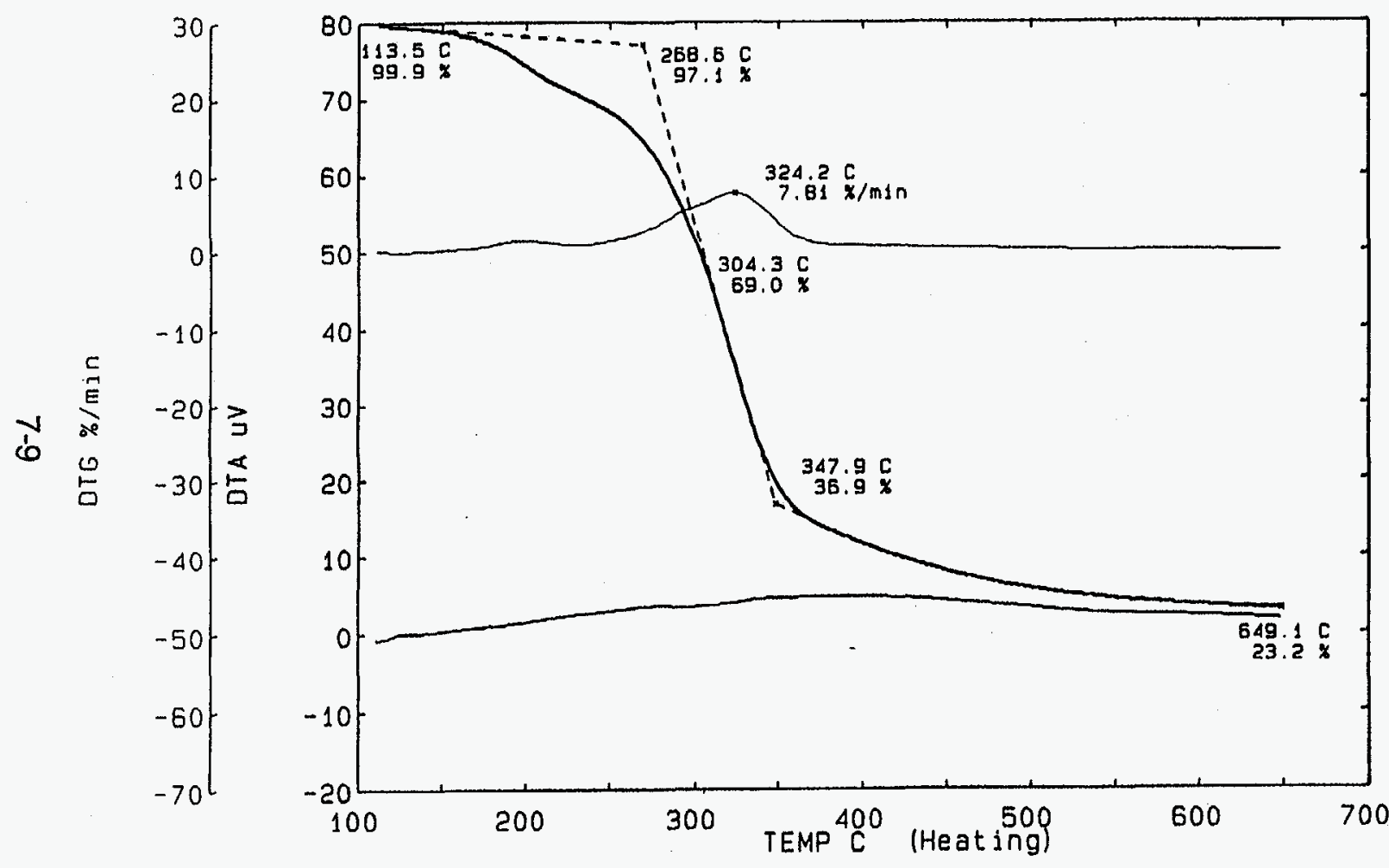

\begin{tabular}{|c|c|}
\hline $\begin{array}{l}00 \\
90\end{array}$ & $\begin{array}{c}\text { Sorghum Stored for zero weeks } \\
\text { (Sorghum bicolor) }\end{array}$ \\
\hline 80 & Heating rate- ${ }^{\circ} \mathrm{C} / \mathrm{min}: 10$ \\
\hline \multirow[t]{2}{*}{70} & Sample weight-mg: 3.947 \\
\hline & $\mathrm{T}_{\text {wndut }}{ }^{-\mathrm{C}} \mathrm{C}: 113.5$ \\
\hline 60 & Volatilization \\
\hline 50 & $T_{\text {tunnpolat }}{ }^{\circ} \mathrm{C}: 268.6$ \\
\hline \multirow{2}{*}{$40 \stackrel{\infty}{5}$} & $\mathrm{~T}_{\text {mL. pols }} 0^{\circ} \mathrm{C}: 304.3$ \\
\hline & $\mathrm{T}_{\text {endpolins }}{ }^{\circ \mathrm{C}}: \mathbf{3 4 7 . 9}$ \\
\hline \multirow[t]{2}{*}{30} & $T_{\operatorname{mex}}{ }^{\circ}{ }^{\circ}:=324.2$ \\
\hline & Rate $_{\operatorname{mex}}-\mathrm{mg} / \mathrm{min}: 7.81$ \\
\hline 20 & Wt. $\%_{\text {oss } c^{-:}: 23.2}$ \\
\hline \multirow[t]{2}{*}{10} & $\Delta \mathrm{H}_{\mathrm{vol} 1}, \mu \mathrm{v}, \mathrm{sec} / \mathrm{mg}: \mathrm{NS}$ \\
\hline & Proximate analysis \\
\hline \multirow[t]{5}{*}{0} & Volatiles $_{\text {Toul }}-w t \%: 77.8$ \\
\hline & Volatiles $_{650} c^{-w t} \%: 76.8$ \\
\hline & Volatiles $_{\text {ssa-9so }} c^{-w t} \%: 1.0$ \\
\hline & Fixed carbon-wt $\%: 21.6$ \\
\hline & Ash-wt\%: 0.6 \\
\hline
\end{tabular}



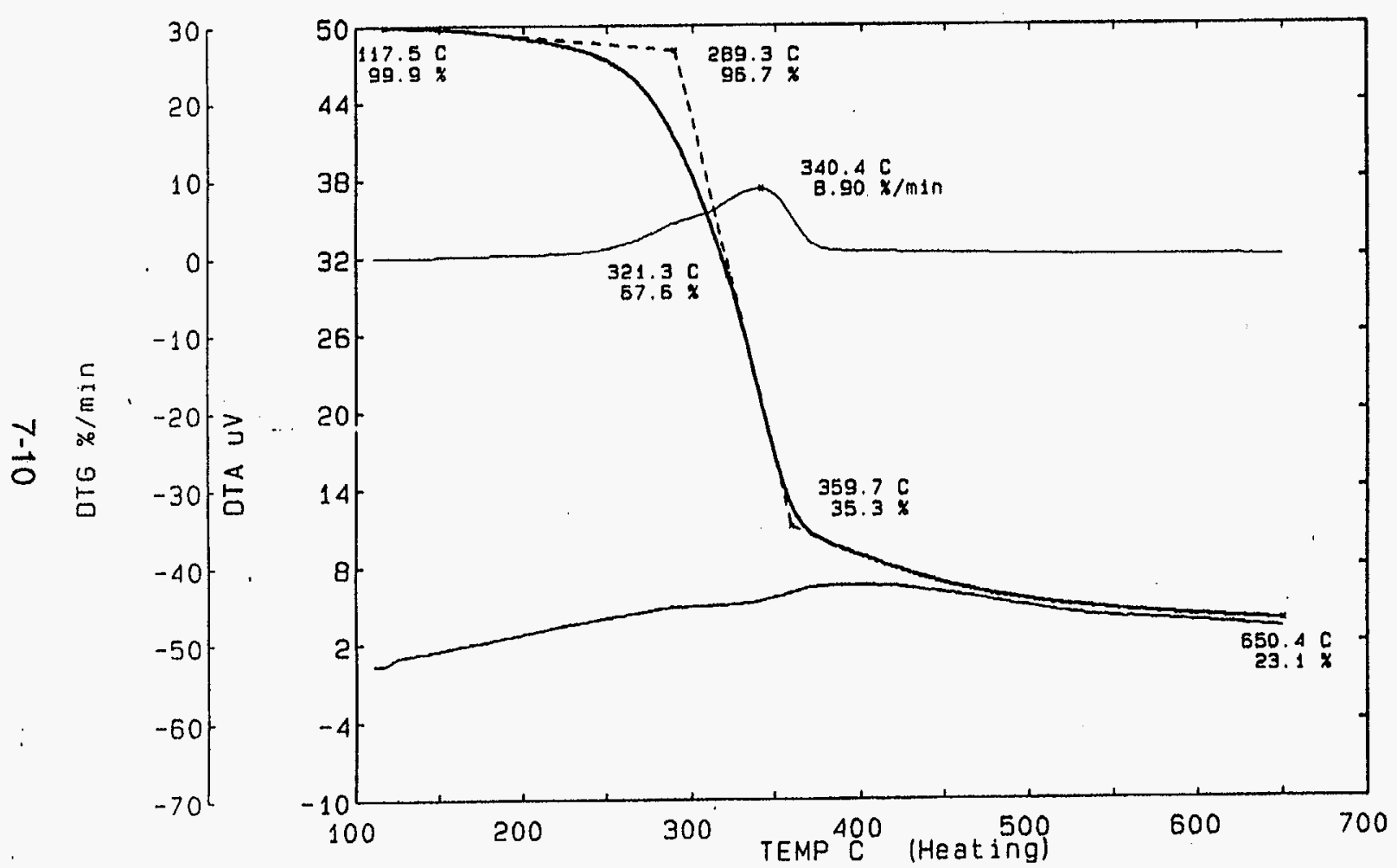

100
90
80
70
60
50
40
30
20
10
0

\section{Sorghum Stored for 26 weeks}

(Sorghum bicolor)

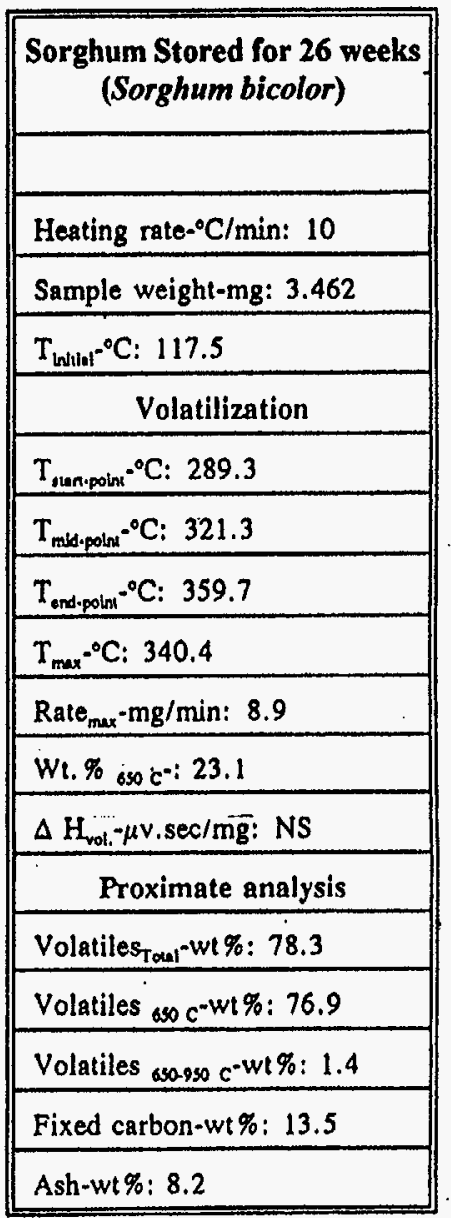



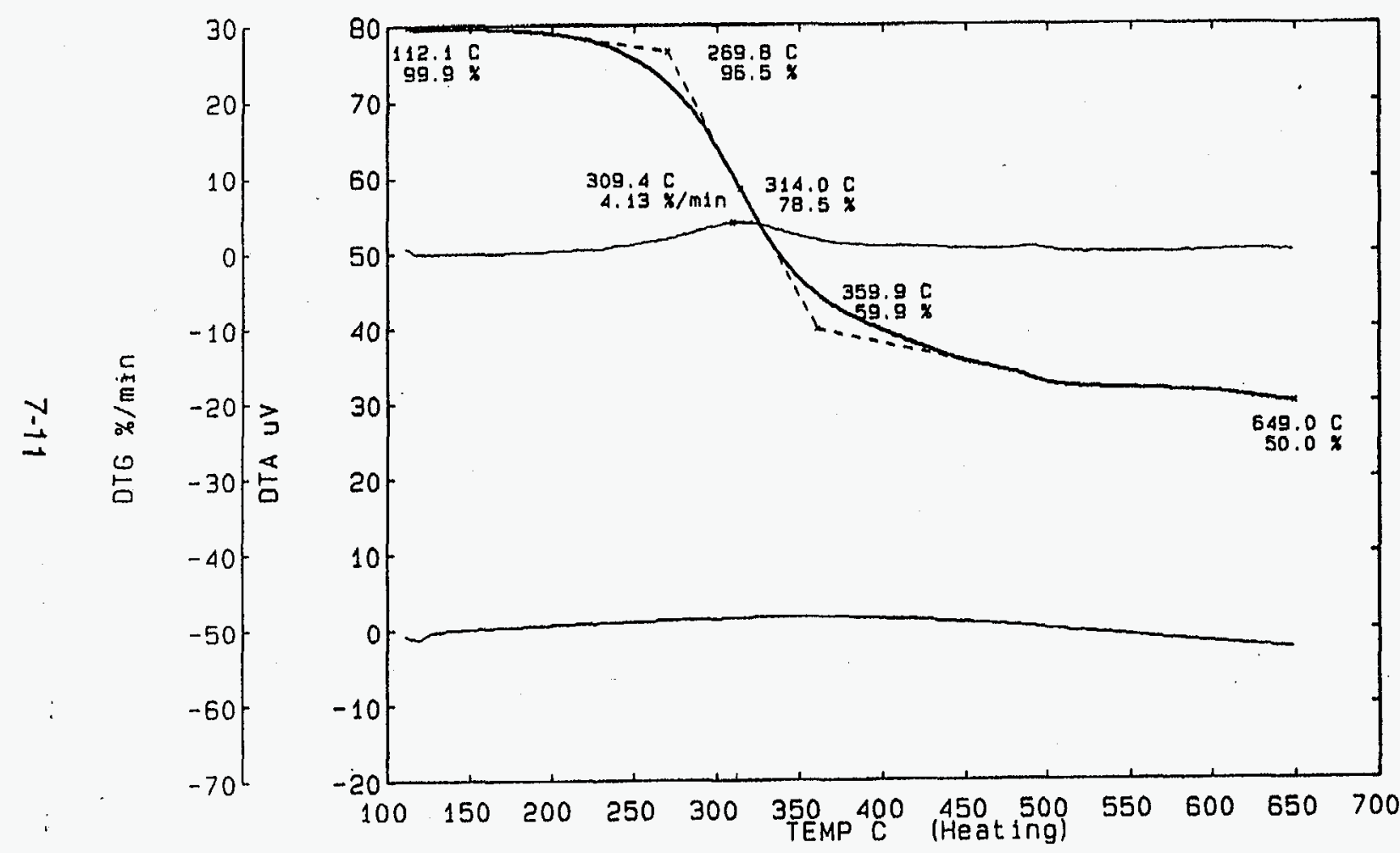

\begin{tabular}{|c|c|}
\hline 90 & $\begin{array}{l}\text { Spinach stem Xylem and } \\
\text { Phloem } \\
\text { (Spinacea oleracea) }\end{array}$ \\
\hline \multirow{2}{*}{80} & \\
\hline & Heating rate- $-^{\circ} \mathrm{C} / \mathrm{min}: 10$ \\
\hline 70 & Sample weight-mg: 0.929 \\
\hline \multirow{2}{*}{60} & 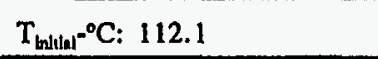 \\
\hline & Volatilization \\
\hline 50 & $T_{\text {santpotnn }}{ }^{-0} \mathrm{C}: 269.8$ \\
\hline \multirow[t]{2}{*}{$40 \stackrel{0}{0}$} & $T_{\text {mblpolyx }}{ }^{-\mathrm{C}}: 314.0$ \\
\hline & $\mathrm{T}_{\text {ent.pont }}{ }^{-} \mathrm{C}: \quad 359.9$ \\
\hline 30 & $\mathrm{~T}_{\max }{ }^{-0} \mathrm{C}: 309.4$ \\
\hline \multirow{2}{*}{20} & Rate $_{\operatorname{mex}}-\mathrm{mg} / \mathrm{min}: 4.13$ \\
\hline & Wit. $\%_{0 S 0} c^{-:}: 50.0$ \\
\hline 10 & $\Delta H$ vol. $-\mu ; v . s e c / m g:$ NS \\
\hline \multirow[t]{6}{*}{0} & Proximate analysis \\
\hline & Volatiles $_{\text {Tow }}-w t \%: 53.7$ \\
\hline & Volatiles $_{6 s 0 \mathrm{c}} \mathrm{c}^{-\mathrm{w}} \%: 50.0$ \\
\hline & Volatiles $_{s s 0-950} c^{-w t} \%: 3.7$ \\
\hline & Fixed carbon-wt \%: 11.8 \\
\hline & Ash-wt\%: 34.5 \\
\hline
\end{tabular}

\section{Spinach stem Xylem and Phloem} (Spinacea oleracea) 

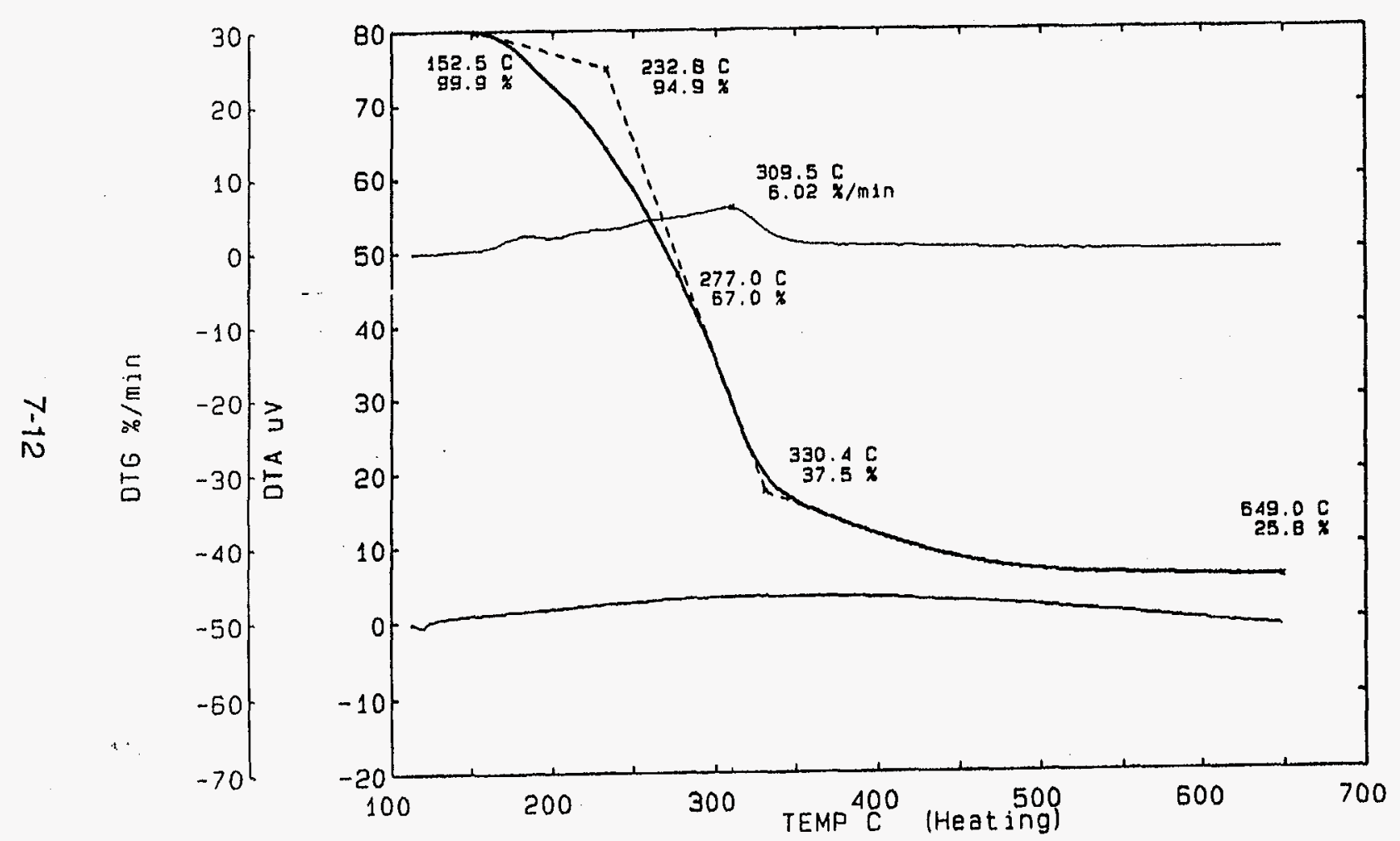

\begin{tabular}{|c|c|}
\hline 90 & $\begin{array}{l}\text { Spinach stem cell Chloroplast } \\
\text { (Spinacea oleracea) }\end{array}$ \\
\hline \multirow{2}{*}{80} & \\
\hline & Heating rate $-^{\circ} \mathrm{C} / \mathrm{min}: 10$ \\
\hline 70 & Sample weight-mg: 0.888 \\
\hline \multirow{2}{*}{60} & $T_{\text {bllitio }}^{-0} \mathrm{C}: 152.5$ \\
\hline & Volatilization \\
\hline 50 & $T_{\text {tunn,potnx }}{ }^{\circ} \mathrm{C}: 232.8$ \\
\hline \multirow{2}{*}{$40 \Leftrightarrow$} & $T_{\text {mbl-polx }}{ }^{\circ} \mathrm{C}: 277.0$ \\
\hline & $T_{\text {end.potax }}{ }^{\circ} \mathrm{C}: 330.4$ \\
\hline 30 & $\mathrm{~T}_{\text {max }}{ }^{\circ} \mathrm{C}: 309.5$ \\
\hline \multirow{2}{*}{20} & Rate $_{\max }-\mathrm{mg} / \mathrm{min}: 6.02$ \\
\hline & $W_{1 .} \%_{0 s 0} c^{-:} 25.8$ \\
\hline 10 & $\Delta H$ vol. $-\mu, v . s e c / m g:$ NS \\
\hline \multirow{6}{*}{0} & Proximate analysis \\
\hline & Volatiles $_{\text {Tout }}-\mathrm{w1} \%: 76.9$ \\
\hline & Volatiles $_{\text {so }} \mathrm{c}^{-\mathrm{w}} \mathrm{w} \%: 74.2$ \\
\hline & Volatiles $_{\text {sso. }}$ sso $c^{-w t} \%: 2.7$ \\
\hline & Fixed carbon-wt\%: 21.6 \\
\hline & Ash-wt\%: 1.5 \\
\hline
\end{tabular}

\section{Spinach stem cell Chloroplast}

(Spinacea oleracea) 

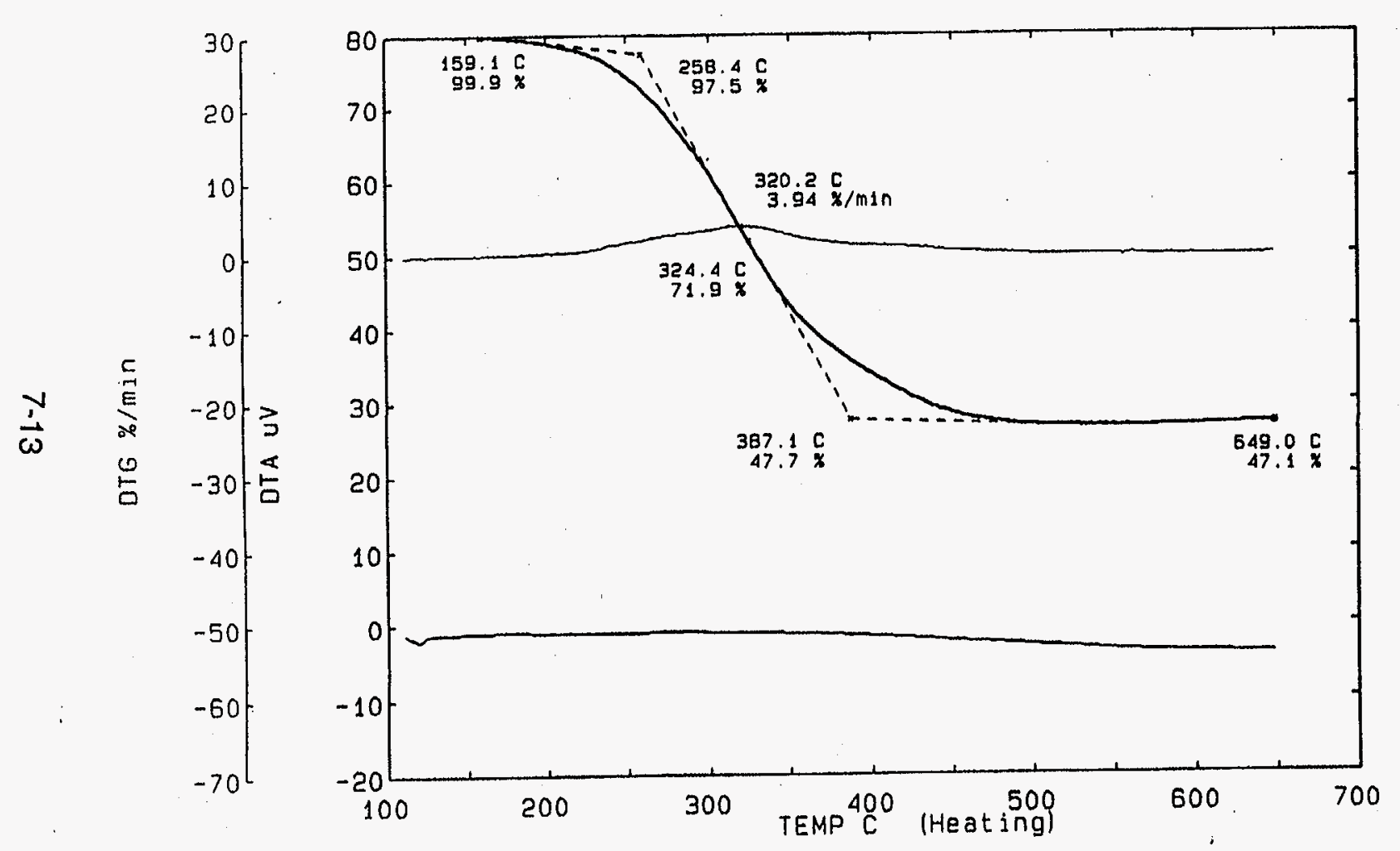

\begin{tabular}{|c|c|}
\hline 90 & $\begin{array}{l}\text { Spinach cell } \\
\text { Cytoplasmic liquid . } \\
\text { (Spinacea oleracea) }\end{array}$ \\
\hline BO & Heating rate- ${ }^{\circ} \mathrm{C} / \mathrm{min}: 10$ \\
\hline 70 & Sample weight-mg: \\
\hline \multirow{2}{*}{60} & $\mathrm{~T}_{\text {inidus }}$ - $^{\circ} \mathrm{C}: 159.1$ \\
\hline & Volatilization \\
\hline \multirow{3}{*}{$\begin{array}{ll}50 \\
40 \stackrel{0}{0}\end{array}$} & $\mathrm{~T}_{\text {aun-polxt }}{ }^{\circ} \mathrm{C}: 258.4$ \\
\hline & $T_{\text {mddpolmx }}{ }^{-\mathrm{C}:} \quad 324.4$ \\
\hline & $T_{\text {exd.poth }}{ }^{-0} \mathrm{C}: 649.0$ \\
\hline 30 & $T_{\max }{ }^{-0} \mathrm{C}: 320.2$ \\
\hline \multirow{2}{*}{20} & Rate $_{\operatorname{mex}}-\mathrm{mg} / \mathrm{min}: 3.94$ \\
\hline & Wt. $\%_{650} c^{-:}: 47.1$ \\
\hline \multirow[t]{2}{*}{10} & $\Delta H$ vol. $-\mu ;$ v.sec/mg: NS \\
\hline & Proximate analysis \\
\hline \multirow{5}{*}{0} & Volatiles $_{\text {Tout }}-w 1 \%$ : \\
\hline & Volatiles $_{\text {oso }} \mathrm{c}^{-\mathrm{w} t \%: 52.9}$ \\
\hline & Volatiles $_{650.950} c^{-w t} \%:$ \\
\hline & Fixed carbon-wt\%: \\
\hline & Ash-wt\%: \\
\hline
\end{tabular}

Spinach cell Cytoplasmic liquid

(Spinacea oleracea) 

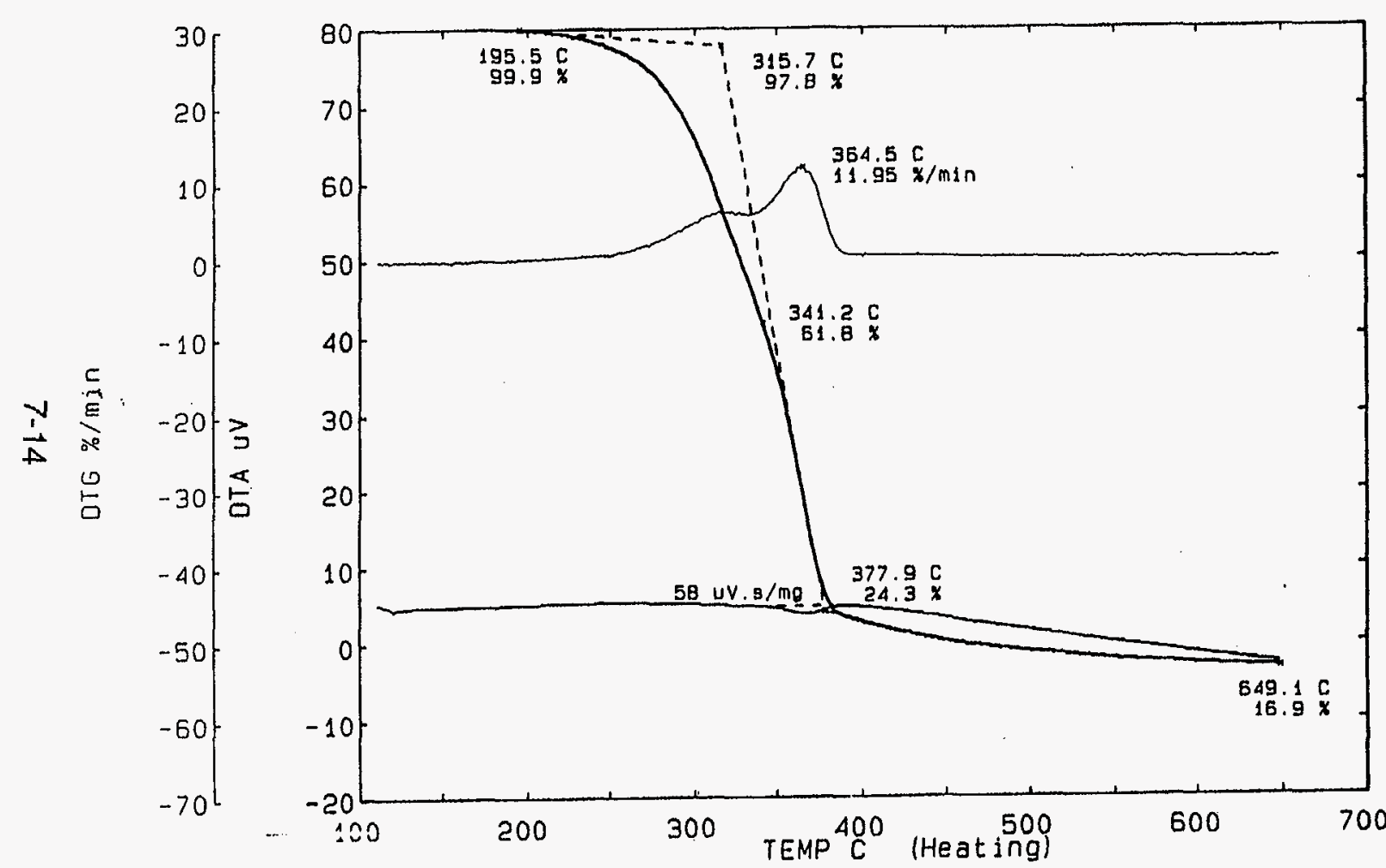

\begin{tabular}{|c|c|}
\hline 100 & $\begin{array}{c}\text { Sugar cane baggase stored for } \\
\text { zero weeks } \\
\text { (Saccharum officinarum) }\end{array}$ \\
\hline 80 & Heating rate- ${ }^{\circ} \mathrm{C} / \mathrm{min}: 10$ \\
\hline \multirow[t]{2}{*}{70} & Sample weight-mg: 2.480 \\
\hline & $T_{\text {lnduat }}{ }^{\circ} \mathrm{C}: 195.5$ \\
\hline 60 & Volatilization \\
\hline \multirow{3}{*}{$\begin{array}{l}50 \\
40 \stackrel{2}{\circ}\end{array}$} & $\mathrm{T}_{\text {our poins }}{ }^{\circ} \mathrm{C}: 315.7$ \\
\hline & 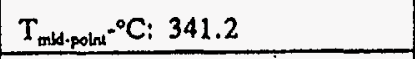 \\
\hline & $\mathrm{T}_{\text {end.polot: }}{ }^{\circ} \mathrm{C}: 377.9$ \\
\hline 30 & $\mathrm{~T}_{\text {max }}{ }^{-0} \mathrm{C}: 364.5$ \\
\hline \multirow{2}{*}{20} & Rate $_{\text {max }}-\mathrm{mg} / \min : 11.95$ \\
\hline & Wt. \% $600 c^{-:}: 16.9$ \\
\hline \multirow[t]{2}{*}{10} & $\Delta \mathrm{H}_{\mathrm{vol}}-\mu \mathrm{v} . \mathrm{sec} / \mathrm{mg}: 58$ \\
\hline & Proximate analysis \\
\hline \multirow[t]{2}{*}{0} & Volatiles $_{\text {Toul }}-$ wt $\%: 83.5$ \\
\hline & Volatiles sso c-wi\%: 83.1 \\
\hline \multirow{3}{*}{ exs } & 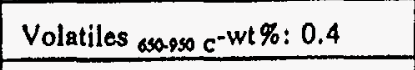 \\
\hline & Fixed carbon-wt\%: 14.7 \\
\hline & Ash-wt\%: 1.8 \\
\hline
\end{tabular}

Sugar cane baggase stored for zero weeks

(Saccharum officinarum) 

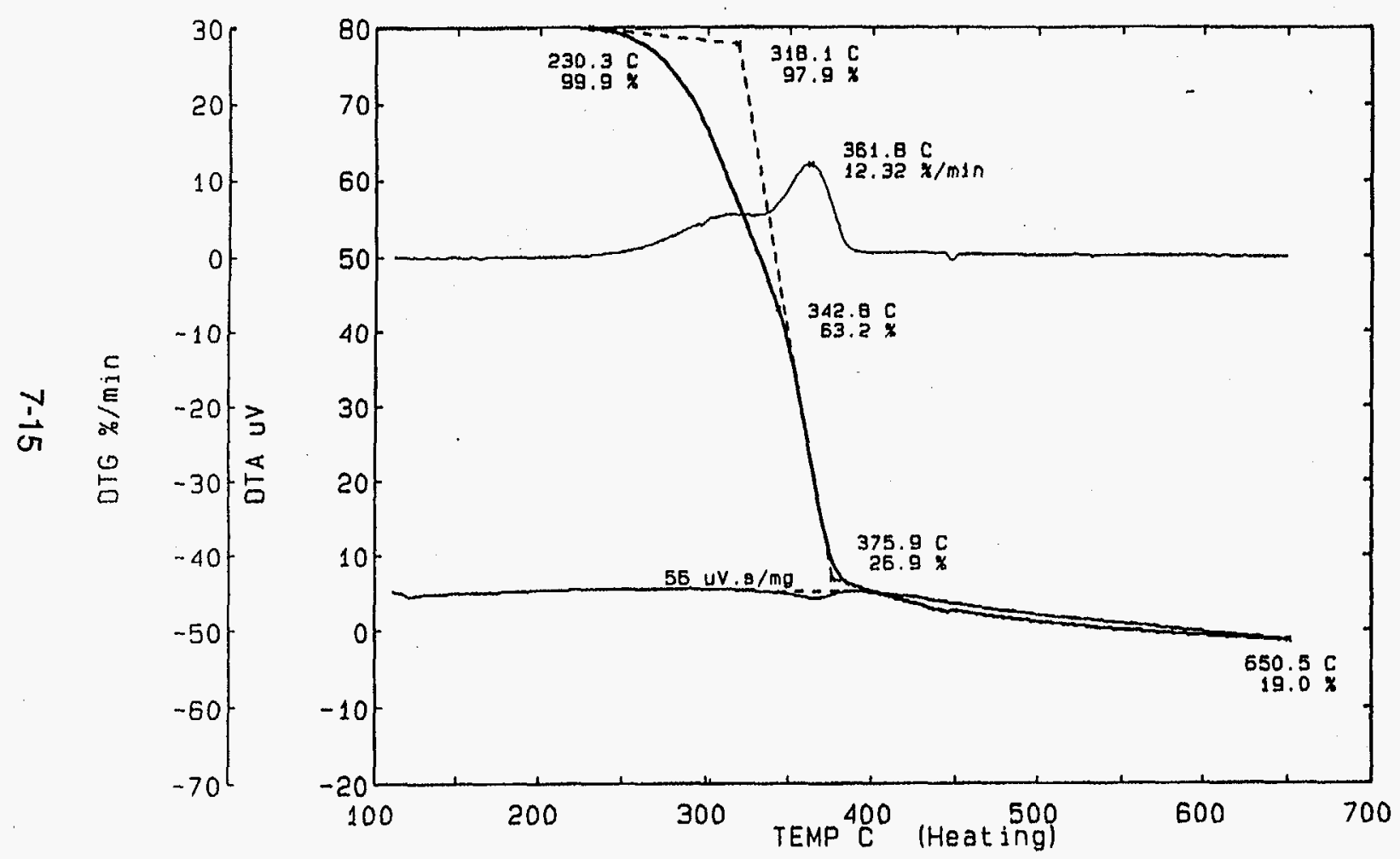

\begin{tabular}{|c|c|}
\hline 90 & \begin{tabular}{|c|} 
Sugar cane baggase stored for \\
26 weeks \\
(Saccharum officinarum) \\
\end{tabular} \\
\hline 80 & Heating rate- ${ }^{\circ} \mathrm{C} / \mathrm{min}: 10$ \\
\hline 70 & Sample weight-mg: 2.711 \\
\hline \multirow{2}{*}{60} & $\mathrm{~T}_{\text {Inulat } 1^{\circ} \mathrm{C}: 230.3}$ \\
\hline & Volatilization \\
\hline \multirow{3}{*}{$\begin{array}{ll}50 \\
40 \stackrel{9}{2}\end{array}$} & $T_{\text {sun:polet }}{ }^{\circ} \mathrm{C}: 318.1$ \\
\hline & $T_{\text {mbl-polate }}{ }^{\circ} \mathrm{C}: 342.8$ \\
\hline & $\mathrm{T}_{\text {end.polnt }}{ }^{\circ} \mathrm{C}: 375.9$ \\
\hline 30 & $T_{\text {max }}={ }^{\circ} \mathrm{C}: 361.8$ \\
\hline \multirow{2}{*}{20} & Rate $_{\max }=\mathrm{mg} / \min : 12.32$ \\
\hline & Wt. \% osoc: 19.0 \\
\hline 10 & $\Delta H_{\text {vol. }}-\mu v .8 \mathrm{ec} / \mathrm{mg}: 56$ \\
\hline \multirow{3}{*}{0} & Proximate analysis \\
\hline & Volatiles $_{\text {Toul-wt }}$ - $: 81.5$ \\
\hline & Volatiles ${ }_{\text {sos }} \mathrm{c}^{-\mathrm{wt}} \%: 81.0$ \\
\hline \multirow[t]{3}{*}{$\mathbf{K S}$} & Volatiles ssa-9so $c^{-w 1 \%: 0.5}$ \\
\hline & Fixed carbon-wt\%: 16.7 \\
\hline & Ash-wt\%: 1.8 \\
\hline
\end{tabular}

\section{Sugar cane baggase stored for 26 weeks}

(Saccharum officinarum) 

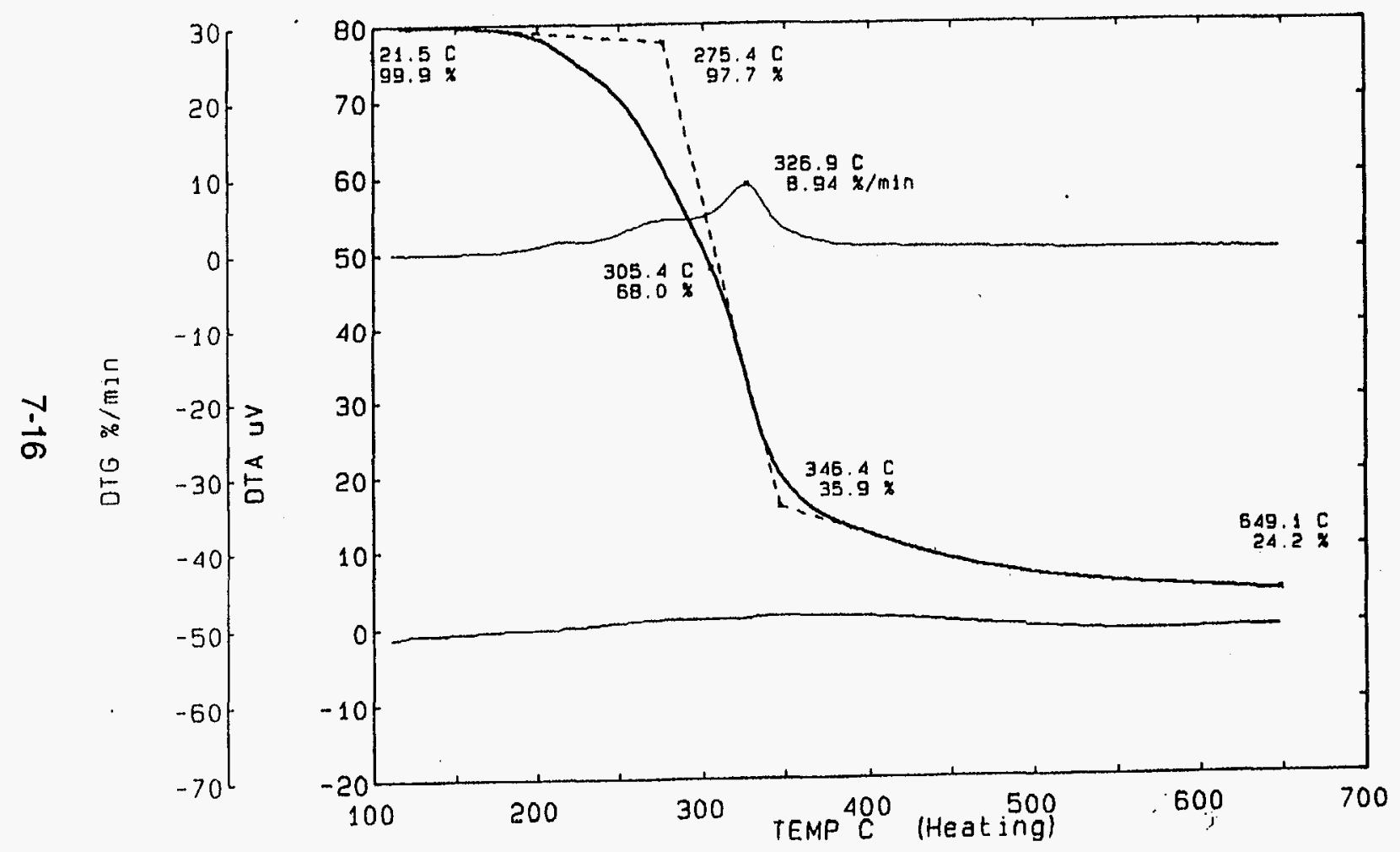

100

\section{Switchgrass Stored for Zero Weeks}

(Panicum virgatum L.) 


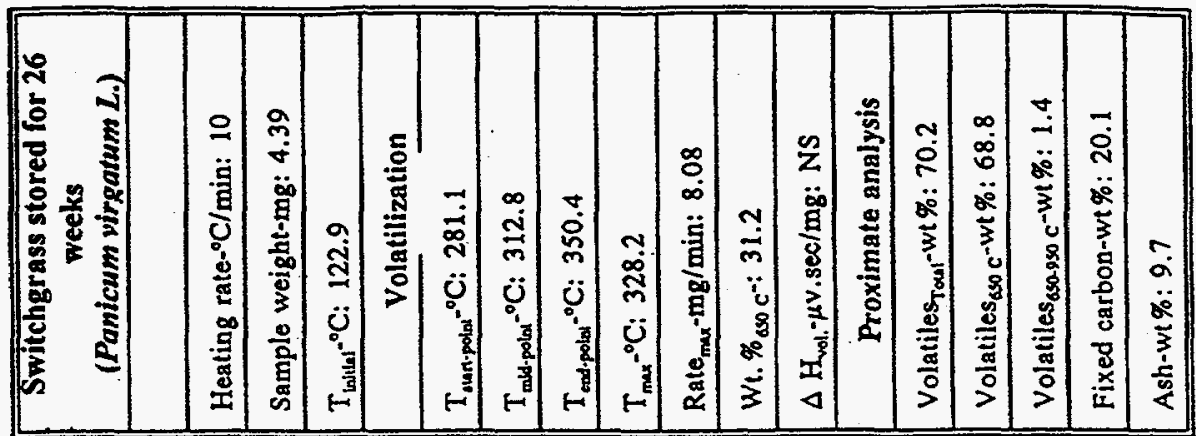

* 91

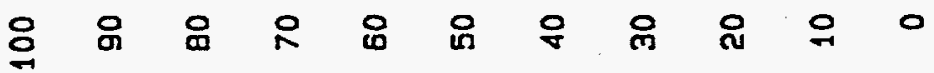
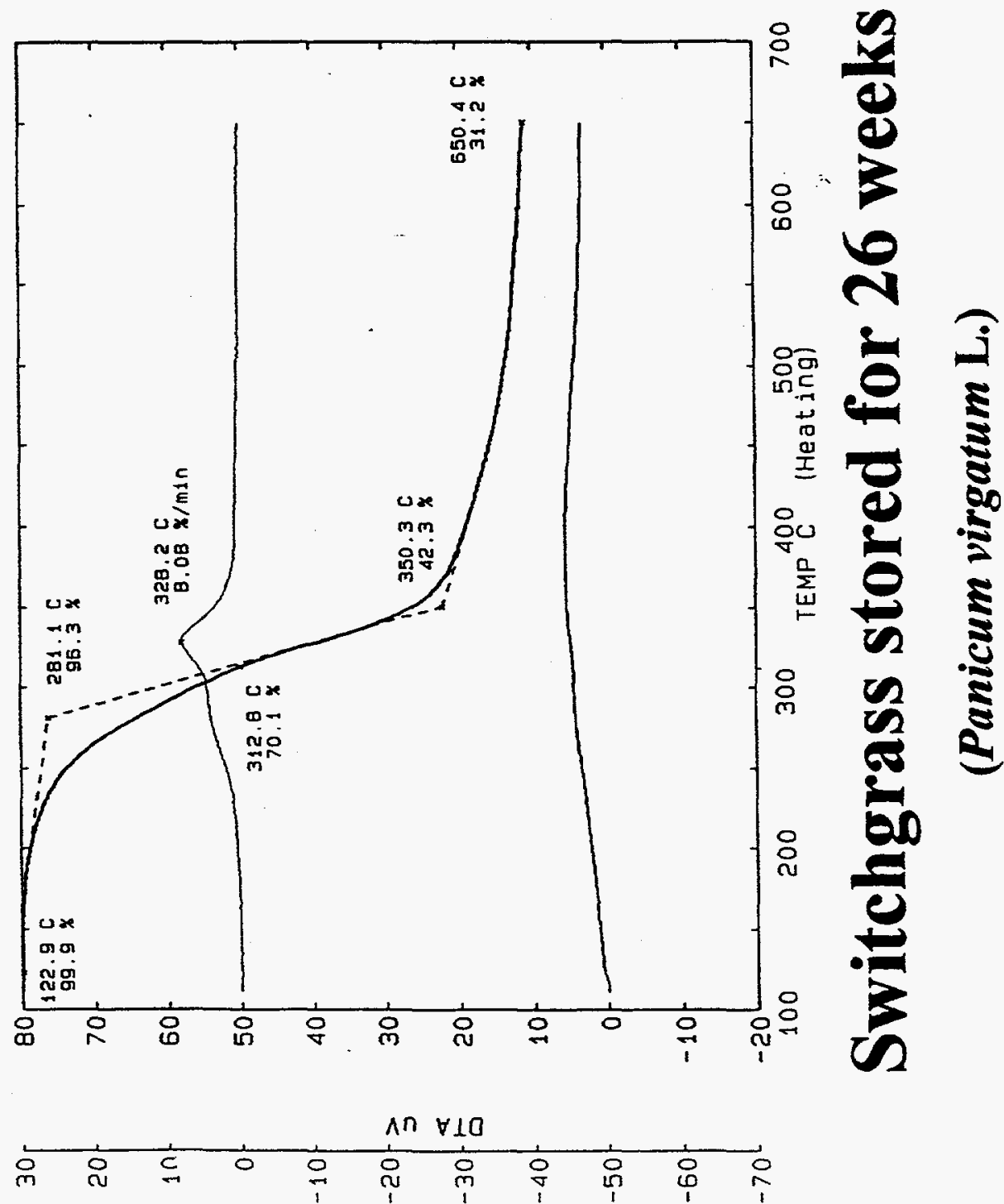

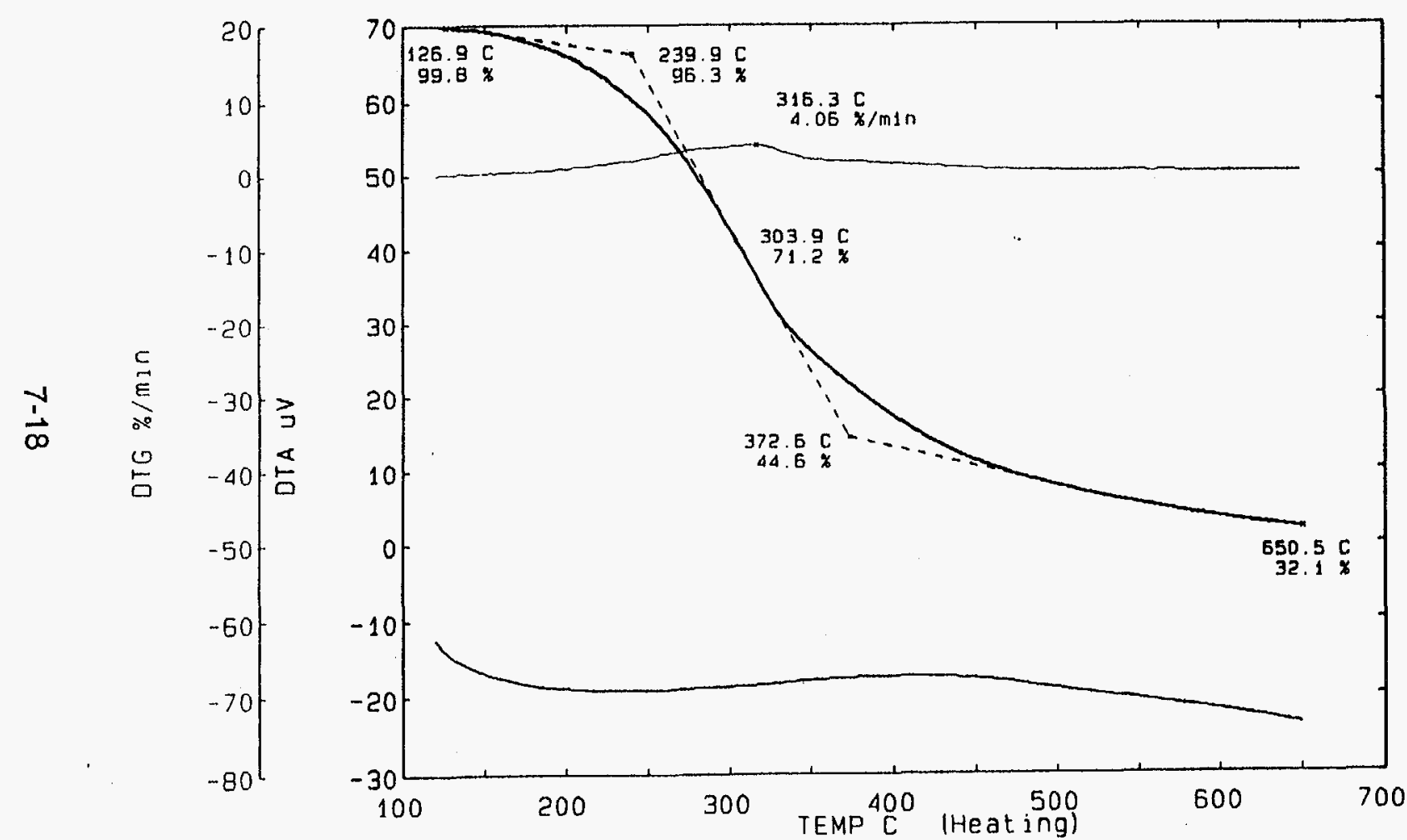

\begin{tabular}{|c|c|}
\hline $\begin{array}{r}100 \\
90\end{array}$ & $\begin{array}{l}\text { Brown Rotted Wood } \\
\text { (S. pine by P. placenta) }\end{array}$ \\
\hline \multirow{2}{*}{80} & Heating rate. $\cdot^{\circ} \mathrm{C} / \mathrm{min}: 10$ \\
\hline & Sample weight-mg: 7.657 \\
\hline 70 & $\mathrm{~T}_{\text {inimit1 }}{ }^{\circ} \mathrm{C}: 126.9$ \\
\hline \multirow{2}{*}{60} & Volatilization \\
\hline & $\mathrm{T}_{\text {dunn.poime }}{ }^{-0} \mathrm{C}: 239.9$ \\
\hline \multirow{3}{*}{ 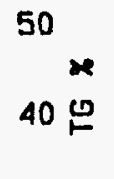 } & $T_{\text {mad.polm }}{ }^{-0} \mathrm{C}: 303.9$ \\
\hline & $T_{\text {ent.poim }}{ }^{-0} \mathrm{C}: 372.6$ \\
\hline & $\mathrm{T}_{\operatorname{mx}}{ }^{\circ} \mathrm{C}: 316.3$ \\
\hline 30 & $\mathrm{Rate}_{\max }-\mathrm{mg} / \mathrm{min}: 4.06$ \\
\hline \multirow[t]{2}{*}{20} & Wt. $\%$ ossoc: 32.1 \\
\hline & $\Delta H_{\text {vol. }} \cdot \mu v . \sec / \mathrm{mg}: \mathrm{NS}$ \\
\hline 10 & Proximate analysis \\
\hline \multirow{5}{*}{0} & Volatiles $_{\text {Tout }}-w 1 \%: 72.8$ \\
\hline & Volatiles $_{\Delta s 0} \mathrm{c}^{-\mathrm{wt}} \%: 67.9$ \\
\hline & Volatiles $_{580.950} c^{-w 1} \%: 4.9$ \\
\hline & Fixed carbon-w1\%: 3.8 \\
\hline & Ash-wt\%: 23.4 \\
\hline
\end{tabular}



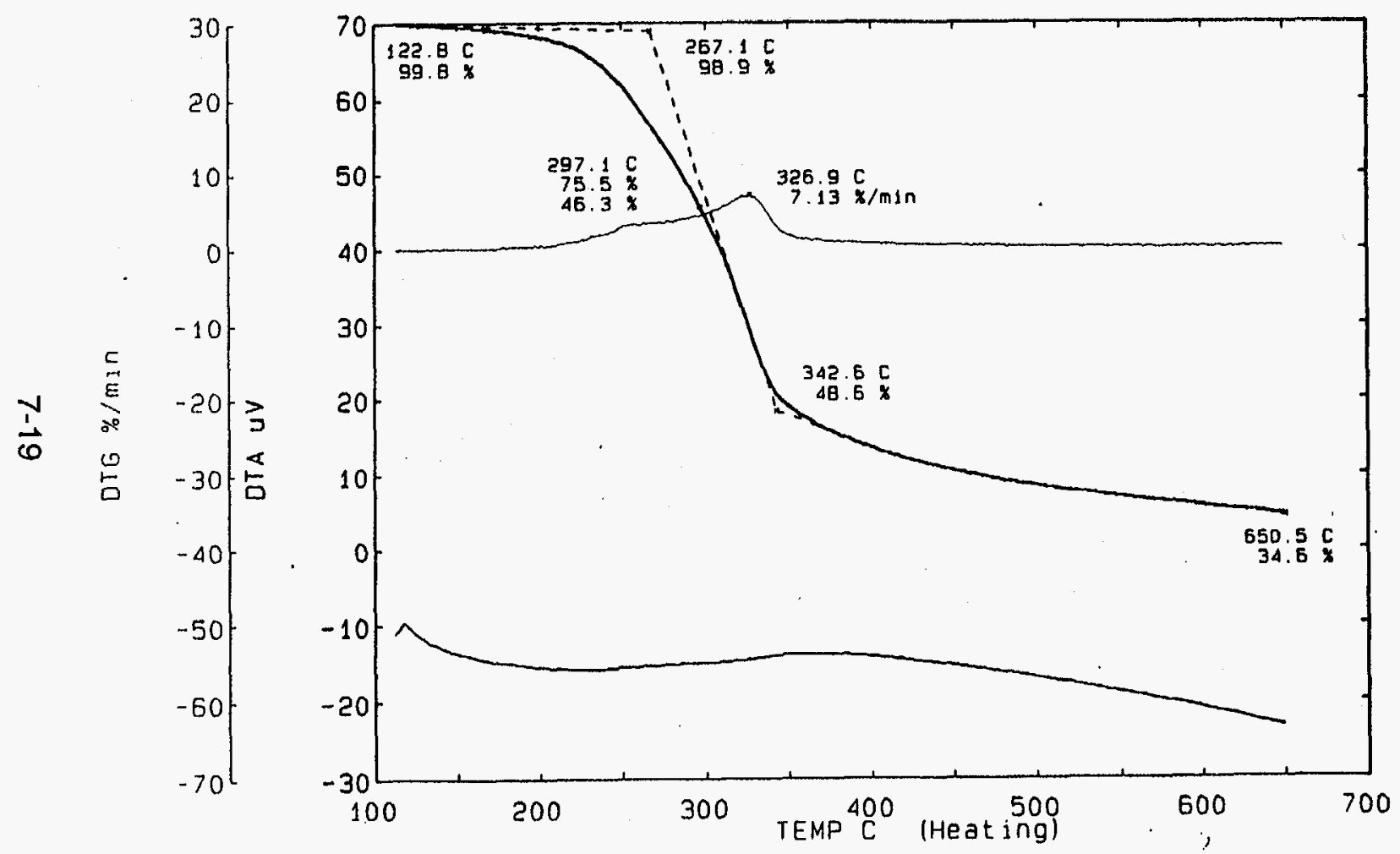

\begin{tabular}{|c|c|}
\hline 100 & White rotten wood \\
\hline \multicolumn{2}{|l|}{90} \\
\hline \multirow{2}{*}{80} & Heating rate- ${ }^{\circ} \mathrm{C} / \mathrm{min}: 10$ \\
\hline & Sample weight-mg: 3.176 \\
\hline 70 & $\mathrm{~T}_{\text {inilut }}{ }^{-0} \mathrm{C}: 122.8$ \\
\hline \multirow{2}{*}{60} & Volatilization \\
\hline & $T_{\text {aunipolmt }}{ }^{\circ} \mathrm{C}: 267.1$ \\
\hline \multirow{3}{*}{$\begin{array}{l}50 \\
40 \stackrel{0}{\circ}\end{array}$} & $T_{\text {midpolux }}-^{\circ} \mathrm{C}: 297,1$ \\
\hline & $T_{\text {ent.pext } 0^{\circ} \mathrm{C}:} 342.6$ \\
\hline & $\mathrm{T}_{\max } \mathrm{-}^{-\mathrm{C}} \mathrm{C}: 326.9$ \\
\hline 30 & Rute $_{\max }-\mathrm{mg} / \min : 7.13$ \\
\hline \multirow{2}{*}{20} & $W_{1 .} \%_{\text {asoc }::} 34.6$ \\
\hline & $\Delta H_{v o l}-\mu v . \sec / m g: N S$ \\
\hline \multirow[t]{2}{*}{10} & Proximate analysis \\
\hline & Volatiles $_{\text {Tous }}$-wt\%: 78.5 \\
\hline \multirow{4}{*}{0} & Volatiles $_{\Delta s 0 c^{-w t} \%: 65.4}$ \\
\hline & Volatiles $_{\text {asa-9so }} c^{-w 1 \%: 13.1}$ \\
\hline & Fixed carbon-w1\%: 6.3 \\
\hline & Ash-wt\%: 15.2 \\
\hline
\end{tabular}

\section{White Rotten wood} (White spruce by Ganodermaiaplanatum) 


\section{Chapter 8}

\section{EFFECT OF OPERATING CONDITIONS}

The thermograms shown in this Atlas were taken at one set of conditions except where otherwise noted, and thus are directly comparable. However, the data can be extrapolated to a wider set of conditions. In this chapter the effects of varying the standard experimental conditions are shown so that the user can estimate the effect of various conditions.

\subsection{Effect of heating rate}

The heating rate is the most important factor in determining the temperature at which the decomposition reactions occur. It has been noted by many researchers that an increase in heating rate causes the thermogram to shift towards higher temperatures, and the effect is predicted theoretically in kinetic studies (see Chapter 10). It is important to understand this effect because most of the thermal data is collected at low heating rates while, the engineering application of kinetic parameters obtained from this study may be applied to cases where the heating rate is several orders of magnitude higher or lower.

We have provided thermograms of some samples at different heating rates to enable the user to compare their data with the use of kinetic equations given in chapter 10 . In this example (Western Red Cedar) a tenfold increase in heating rate increases the decomposition temperature about $70^{\circ} \mathrm{C}$.

\subsection{Effect of Sample size}

The sample size is an important aspect in determining the temperature of decomposition. For large sample size, say greater than $10-20 \mathrm{mg}$ for most of the biomass materials at heating rates greater than $10^{\circ} \mathrm{C} / \mathrm{min}$, there is a time lag for the heat transferred from the sample surface to its center. This causes the establishment of a temperature gradient in the sample. This then makes the temperature of the sample measured by the TG less meaningful, because the temperature gradient tends to smear the TG graph to unknown higher temperature. We have shown this effect in a thermogram on a small and large sample. One way to control this effect is to decrease the sample size with the increase in heating rate. This is equivalent to keeping the Biot number of the sample less than 0.1 . This aspect is discussed later in chapter 11 .

\subsection{TG additivity}

It is an unwritten assumption of TG that a mechanical mixture of materials would give a thermogram that would be the arithmetic sum of the proportional quantities of each component. It is not quite so evident that this would occur for the components of biomass which are intimately mixed at the molecular level. It has been shown that kinetic measurements on several components can be added arithmetically to give the observed TG 
curve [Antal, 1994]. Fritsky has shown that The TG curves for several components of MSW can be added arithmetically to give an observed mixture [Fritsky, 1994].

We are indebted to Dr. Agblevor for supplying a sample of the cellulose, lignin and xylan components obtained from populous deltoides. These were mechanically mixed in the proportions in which they are bound together in the natural plant. We show that the thermogram of the both the samples, the natural plant and the component mix are quite similar, if not identical. This suggests that at least in this case there is little or no interaction between the components to affect their temperature of volatilization. 


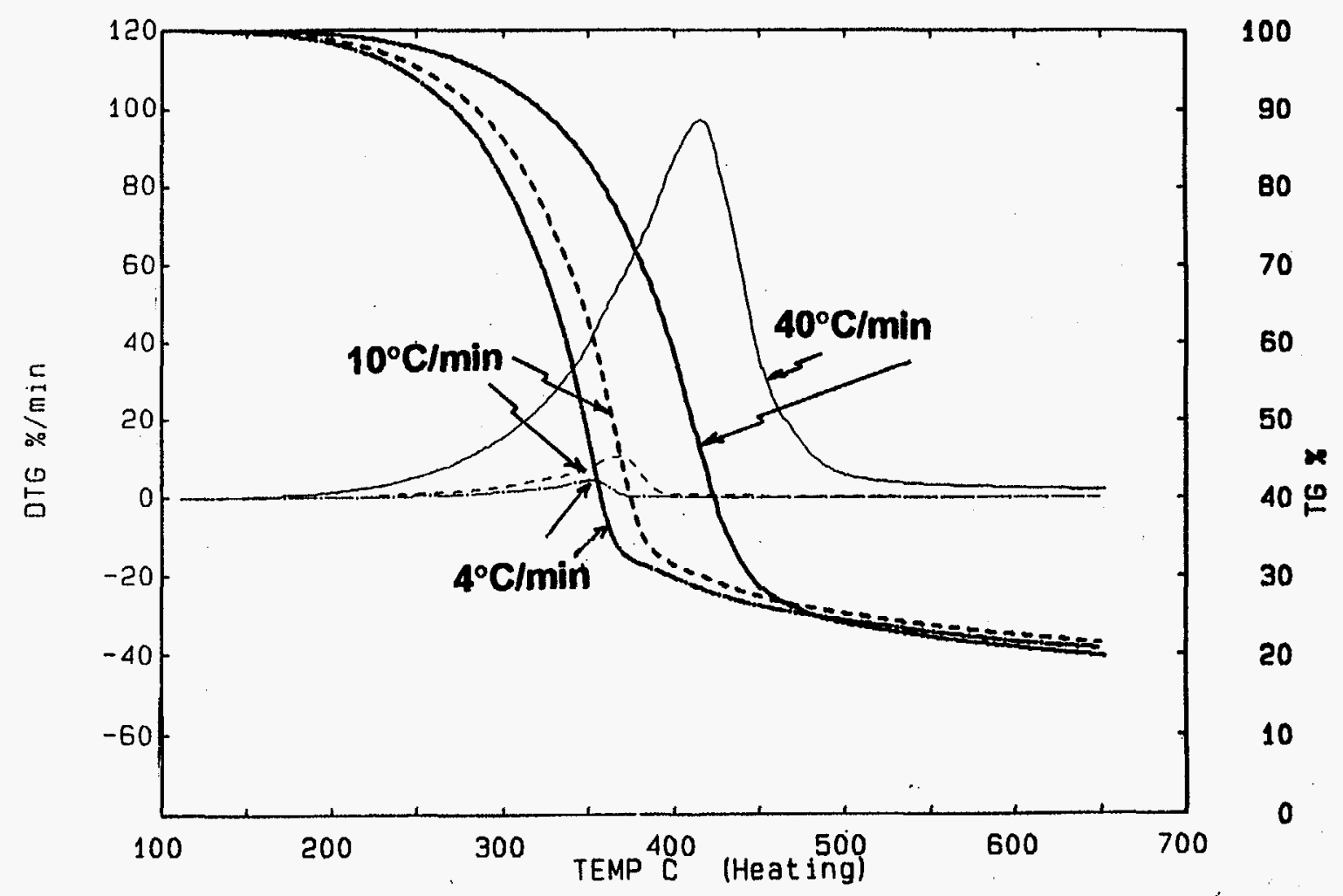

\section{Effect of heating rate} (Western red ceder) 


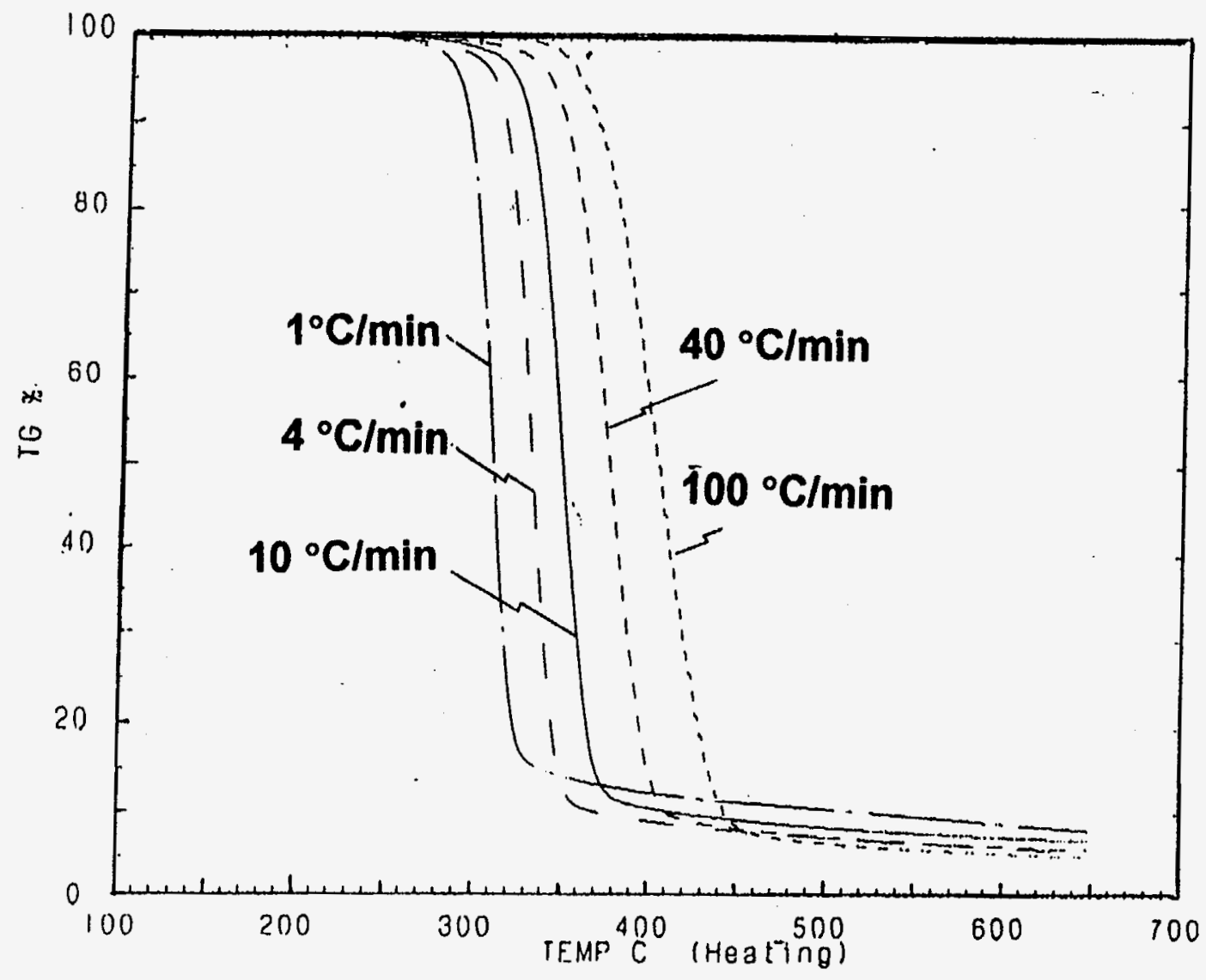

\section{Effect of Heating Rate}




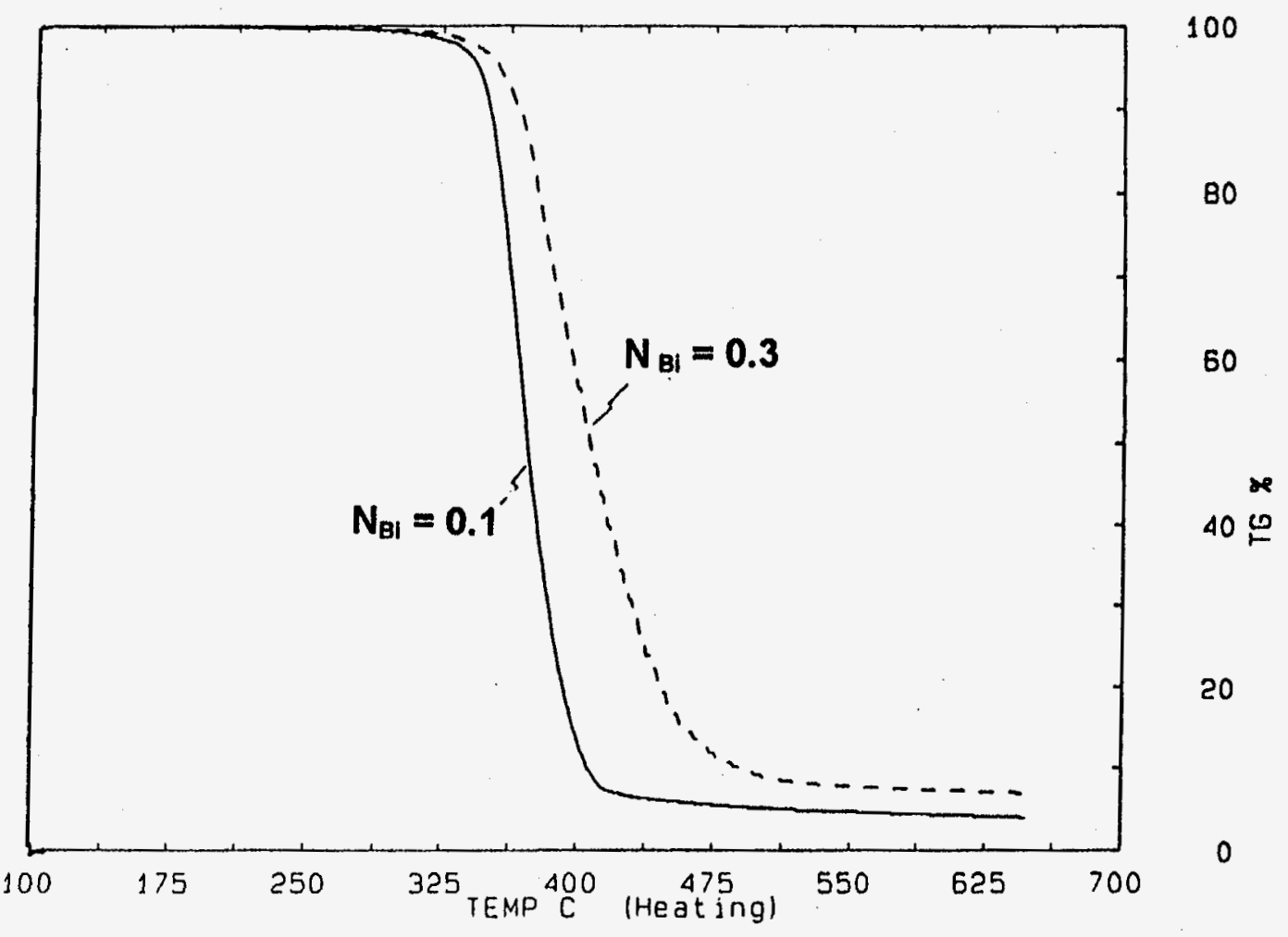

\section{Effect of Particle Size}

Pelletized Baker Cellulose, $5.8 \mathrm{mg}$ and $180 \mathrm{mg}$ with $\mathrm{N}_{B \mathrm{i}}=0.1$ and 0.3 respectively 


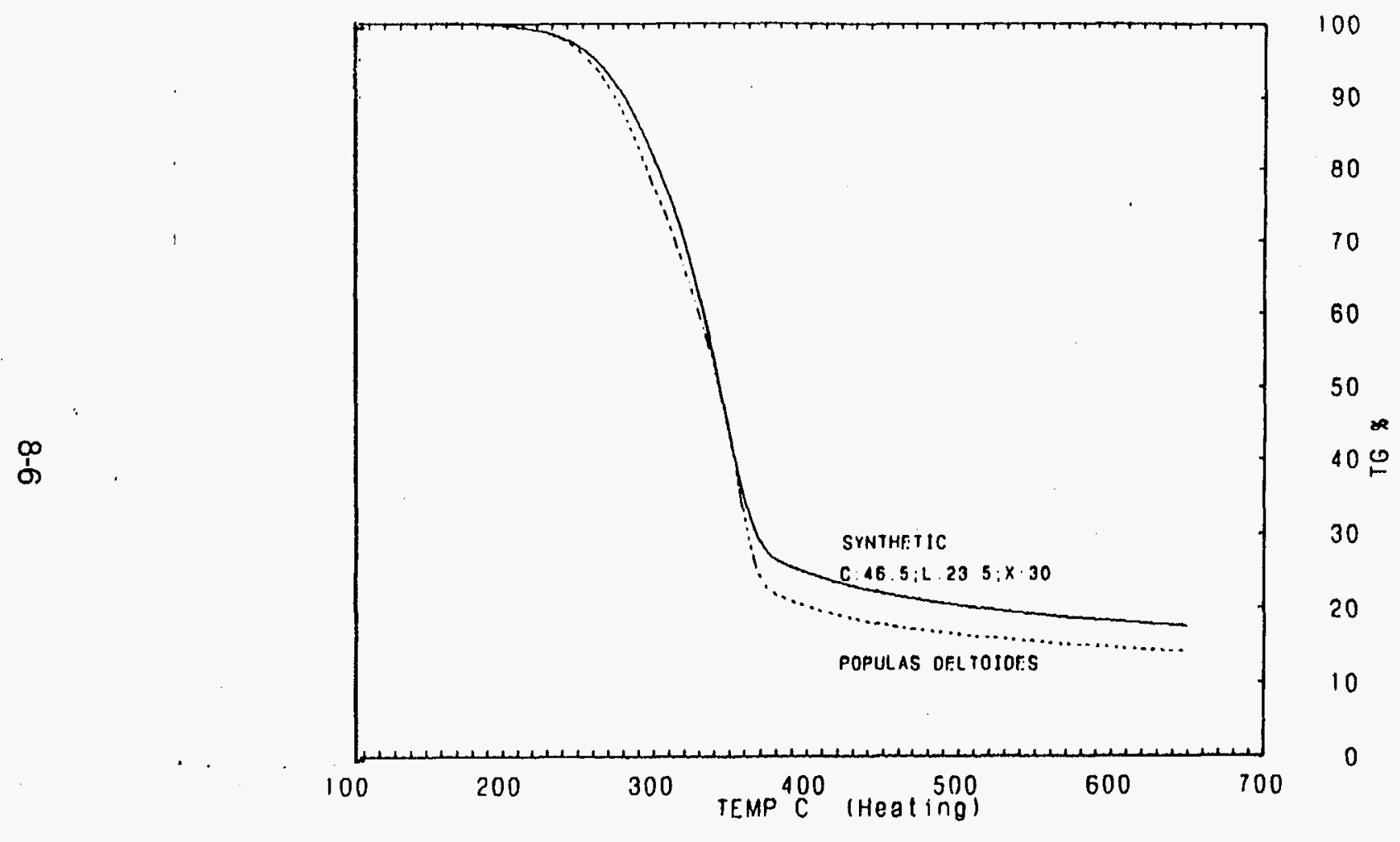

\section{Biomass Component Additivity}

(Populus deltoides - Cell 46.5\%; Lignin 23.5\%; Xylan 30\%) 


\section{Chapter 9}

\section{OVERVIEW OF THERMAL ANALYSIS METHODS}

\subsection{Introduction}

The name thermal analysis (TA), applies to all analytical techniques where the dependence of any physical property of a substance can be related to temperature measurements under controlled conditions. Analytical techniques such as Proximate and Ultimate analysis, Thermometry, Thermogravimetry (TG), Differential thermogravimetry (DTG), Differential thermal analysis (DTA), Differential scanning calorimetry, (DSC), and Thermomechanical analysis (TMA) are all classified under thermal analysis [Wunderlich, 1990].

The proximate analysis is an older technique, originally applied to coal, but it has been widely used for biomass and MSW (ASTM method D3172-73)[ASTM, 1989]. It includes the determination of moisture content, fixed carbon, volatile matter and ash content of the sample. The ultimate analysis was also originally developed for coal and provides the composition of elemental carbon, hydrogen, oxygen, nitrogen and sulfur in a combustible sample (ASTM method D3174-89) [ASTM, 1989].

A given reaction can be followed either by keeping a record of mass change thermogravimetry, and differential thermogravimetry; heat change differential thermal analysis or differential scanning calorimetry; or dimensional change Thermomechanical analysis, as a function of time. The interrelationship of these various methods are illustrated in Figure 9.1.

Thermogravimetry (TG) involves the measurement of mass change during a reaction. The technique is applied to reactions where significant volatilization of sample mass occurs. Thermogravimetry was formerly known as thermogravimetric analysis, or TGA but the International Confederation on Thermal Analysis recommends against using this term.
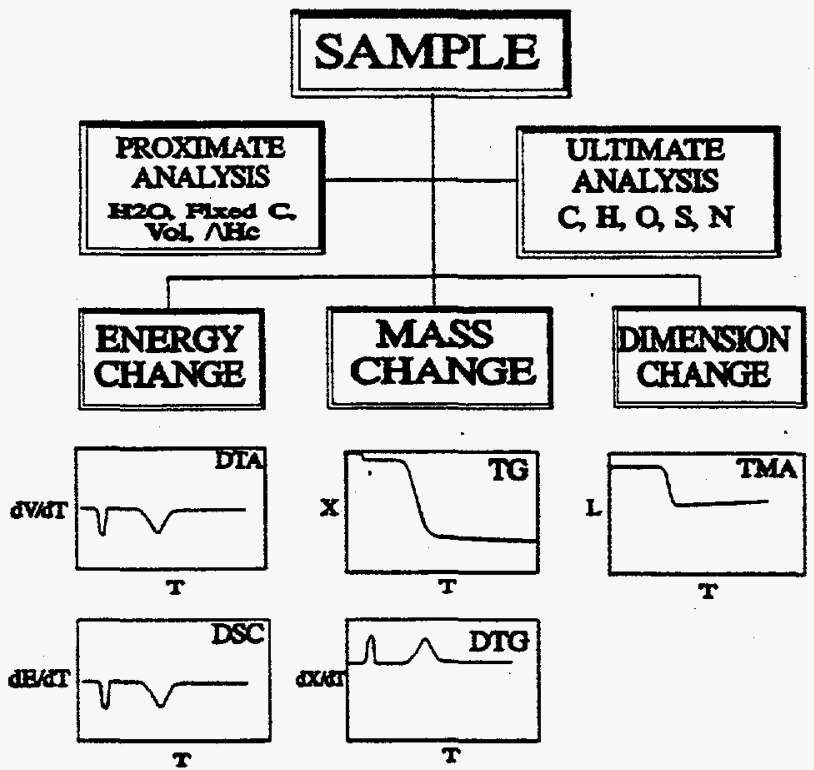

Figure 9.1 Relationship of various thermal techniques 


\subsection{Proximate analysis}

The proximate analysis is a determination of moisture content, fixed carbon, volatile matter and ash content of the sample. It sometimes includes the heat of combustion of the sample, ASTM method (3173-87)[ASTM, 1989].

\subsection{Ultimate analysis}

The ultimate analysis provides the composition of elemental carbon, hydrogen, oxygen, nitrogen and sulfur for a combustible sample (ASTM D3177-84) [ASTM,1989]. While no ultimate analyses were performed on the samples in this atlas, we have included analyses on over 100 samples in Appendix A [Channiwala, 1992].

A number of investigators have found that the elemental composition determined in the ultimate analysis is closely related to the heat of combustion [Tillman, 1978, IGT 1976, Graboski, 1981]. It has recently been found in a survey of these earlier works that the heat of combustion can be predicted from the uitimate analysis according to

$$
H H V(k J / g)=0.3491 \mathrm{C}+1.1783 \mathrm{H}+0.1005 \mathrm{~S}-0.1034 \mathrm{O}-0.0151 \mathrm{~N}-0.0211 \text { Ash }
$$

where HHV is the high heating value of the sample, and $\mathrm{C}, \mathrm{H}, \mathrm{S}, \mathrm{O}, \mathrm{N}$ and Ash are the weight $\%$ of carbon, hydrogen, sulfur, oxygen, nitrogen and ash respectively [Channiwala, 1992]. The average absolute error of prediction is $1.45 \%$, better than that claimed for other correlations, and the bias error for all compounds was found to be negligible which can be approximated to $0 \%$. The accuracy of the ASTM bomb calorimetry method has a reproducibility limit of $240 \mathrm{~J} / \mathrm{g}$, while this correlation for the entire range of fuels offers predictions within $337 \mathrm{~J} / \mathrm{g}$, quite comparable with the measurement uncertainties, particularly considering the widely varying nature of data and the sources of its collection. The proximate, ultimate analysis and heat of combustion data on biomass and some fuel materials samples taken from this work are listed in Appendix A.

\subsection{Thermogravimetry and Differential thermogravimetry}

Isothermal thermogravimetry deals with the change in sample mass at constant temperature as a function of time and was initially used to measure rate constants. In this process a known amount of sample is placed in a furnace in a controlled gaseous atmosphere at the desired temperature. The weight loss of the sample is recorded as a function of time. The graph of weight loss vs. time is referred to as thermogram. It is used to analyze the thermal stability of the sample, the rate of decomposition, the effect of various gases on the decomposition of a sample, possible adsorption and desorption reactions, and to determine the kinetics of the reactions occurring.

Dynamic thermogravimetry is the most common form of thermogravimetric analysis. In this technique a continuous recording of weight change of the sample in a flowing or static gas atmosphere is made as a function of time or temperature at a fixed heating rate, and plotted against temperature. Modern instruments can vary the heating rate over a wide range and hold at various temperatures for specified times, for instance for drying the sample preliminary to making a run. A typical TG trace is shown in the introduction. 
Gravimetric and temperature data are continuously recorded during the course of a TG measurement. Therefore, it is a simple matter of computation to show the first derivative of weight vs. time or temperature, emphasizing the zones of reaction (DTG). The corresponding DTG curve is also shown in the introduction.

TG and DTG have several advantages over isothermal thermogravimetry [Cardwell and Lunar, 1976]. One of the major advantages is that, by conducting one experiment, it is possible to characterize the behavior of a specimen over a very wide temperature range, e.g. $25-1000^{\circ} \mathrm{C}$, thus providing an overview of thermal behavior at all temperatures of interest. It is also possible to determine kinetic parameters of the various reactions over a much wider temperature range than can be studied in isothermal TG by varying the heating rate. Other advantages are elimination of error from sample to sample as one run is used to determine and the reduction of time in performing the kinetic studies.

Another type of thermogravimetry which is not in very common use, is called quasistatic thermogravimetry, in which the sample is heated to constant mass at each of a series of increasing temperatures.

\subsubsection{Applications of thermogravimetric analysis}

Modern day thermogravimetry started in late 1950's with the availability of high quality thermobalances. Now TG is almost universally applied in the field of metallurgy, ceramics, inorganic and organic chemistry, polymers, biochemistry, geochemistry, forensics etc. Some of the applications of thermogravimetry are: thermal decomposition of organic, inorganic and polymeric substances in an inert atmosphere to help in determining the pyrolysis kinetics of decomposition or to determine the thermal stability of the sample; reaction chemistry of solid samples where one of the products is in gaseous state; distillation and evaporation of liquids; proximate analysis; vapor pressure determinations; evaporation and sublimation.

Automatic thermogravimetric analysis developed by Duval determined metallic ion or mixture of ions by the use of thermogravimetry. In this method the crucible is loaded with precipitate. It is then heated in a controlled manner and the mass loss curve is recorded. Once a horizontal plateau is obtained the mass of the precipitate left over is obtained and subsequently from the other plateau obtained at higher temperature the mass of the remaining precipitate is obtained. These mass levels indicate that a definite stoichiometry of the precipitate has been attained [Duval, 1951].

Griffith [1957] has applied thermogravimetry to the determination of the moisture content in the hydrated and anhydrous salts in different phases. The method is based on the fact that under controlled heating rates a selective decomposition of the phase with the highest dissociation temperature takes place. When one phase is decomposed the phase with the next highest decomposition temperature decomposes and so on.

Hoffman [1959] applied thermogravimetry to the analysis of clay and soils. He was able to determine water content, organic content and the inorganic carbonates from pure clays and mixture of clays. The results obtained from TG were in good agreement with those found by $X$-ray diffraction and wet chemical analysis.

TG analysis has also been applied to polymers. Application of TG in this field includes study on the relative thermal stability, the effect of additives on the thermal stability, decomposition 
kinetics, quantitative analysis of various copolymers, oxidation stability, etc. TG has been used to provide information regarding the presence of additives such as plasticizers in the polymer. A thermogram of pure poly vinyl butyryl [Wundlandt and Barbson, 1959] was compared with a one containing plasticizer. The difference between the two thermogram helped in the quantitative determination of plasticizer content.

Recent application of TG has been for the deconvolution of biomass components such as hemicellulose, cellulose and lignin [Varhegyi and Antal, 1993]. This technique is still at the developmental stage.

\subsection{Differential thermal analysis and Differential scanning calorimetry}

Some reactions are endothermic or exothermic but may not involve a weight change. DTA or DSC are applied for detecting endothermicity or exothermicity of reactions.

A technique similar to DTA was first used by Le Chatelier in 1887 for the identification of clay materials and pyrometry. He introduced heat curves for the identification of different clays. This analysis eventually gave rise to differential thermal analysis technique. In his study for the identification of different clay materials Le Chatelier used the rate of deceleration in temperature rise at the point of dehydration, e.g. he found that crystalline Kaolin from Red Mountain Colorado, USA has a deceleration point at $770^{\circ} \mathrm{C}$ and $1000^{\circ} \mathrm{C}$. This characteristic deceleration in temperature was also found in Kaolin from places like France and China. It was observed by Le Chatelier that the adsorption and evolution of heat for a given sample as a function of temperatures is a unique property of that material [Le Chatelier, 1887].

Differential thermal analysis, DTA, is a technique of recording the difference in temperature between a substance and a thermally inert reference material as the two specimens are subjected to identical temperature treatment in an environment heated or cooled at a controlled rate. In this technique the temperature of a sample is compared to the temperature of the thermally inert material as both are heated at a linear heating rate. It is a differential method in that the temperature of the sample $T_{s}$, is compared to the temperature of the reference material $T_{r}$ and the difference between the two is used to determine the heat of reaction. The reference material is usually calcined alumina or a similar inert material. It is known that there are no phase transformations for calcined alumina up to the temperature of $1100^{\circ} \mathrm{C}$. This ensures that the energy changes that are recorded will be due to the transformations taking place in the sample under consideration. DTA does not spell out what net reaction is taking place when an exothermic or endothermic effect is recorded. It simply records that there is a change in energy content taking place and the rate of that change.

The reactions that can be reflected by the DTA curve are: phase transitions; solid state reactions; decompositions; surface reactions and second order transitions, a change in entropy without a change in enthalpy.

Since DTA is based on the measurement of temperature changes in relation to a reference material, the choice of thermocouple and thermocouple placement is an important aspect of the design of DTA instruments [Murphy, 1958].

The amount of reference material used is such that the total heat capacity or mass of the reference material and the sample are about the same, in order to avoid any thermal lags 
between the two thermocouples during the heating of the furnace. Whenever, there is a transition taking place in the sample, the temperature of the sample increases less or more rapidly than that of the reference, depending on the endothermic or exothermic process. This change in heating rate is recorded in terms of temperature difference between the reference and the sample thermocouple. The position of the peaks is related to the chemical change of the substance and the area of the peak is proportional to the energy involved in the reaction occurring. In cases where the entire or the major portion of the sample vaporizes during transition, there can be large deviation between the reference and the sample due to the change in heat capacity of the sample. In such cases it is recommended that the sample be doped in the reference material e.g. Alumina in small quantity so that the heat capacity of both the reference and the sample pans are about the same due to the presence of Alumina in both the pans during the entire range of temperature of interest. This procedure limits the temperature deviation caused due to heating of the sample to a negligible value.

A typical DTA curve is shown in the introduction, showing endothermic peaks, usually from vaporization. The point where a tangent to the side of the peak and to the base line meet is the commencement of the peak. The base lines ideally should be aligned but in practice there is a drift with increasing temperature, due to changes in thermal conductivity or mass.

For an endothermic peak, part of the heat is being used for reaction and part is being used to maintain the sample at constant temperature. At constant specific heat the heat supplied by the source, because of negative value of $\Delta T$, balances the heat being absorbed by the reaction at that point. The whole peak represents an endothermic reaction. If the peak is positive then the whole peak represents an exothermic reaction.

One thing which is evident from examining the endotherm and exotherm is that the heating rate within the sample during the occurrence of a reaction is not the same as that when no reaction is occurring nor is it uniform. This is because the sample temperature lags from that of the reference material which causes the deviation in temperature between the two pans.

Differential Scanning Calorimetry, DSC, is another technique for measuring heats involved in phase changes. In DSC a pilot heater is placed around the sample holder and heat is added or subtracted in small quantities to keep the temperature of the sample and the reference the same. The amount of heat required is monitored and presented graphically. Typical accuracies of heat measurements range from $\pm 10 \%$ to a few tenths of a percent depending on the heating rate employed.

\subsubsection{Applications of differential thermal analysis}

The merit of the DTA curve is that all energy changes occurring in the sample during heating are clearly observable, (provided the sensitivity is high enough) and that the peak areas reflect the energy involved. It can be said with certainty that no two materials give exactly the same complete DTA curve since even slight differences in chemical composition or crystal structure are reflected in differences in either the temperature at which the reaction occurs or the heat of reaction. Because of this property, each substance gives a unique DTA curve for a particular sample. By the selection of a limited number of substances having DTA characteristics very similar to that of unknown samples, it is possible to deconvolute the properties of unknown samples. When this technique is applied to mixtures, sometimes it is 
possible to interpret the entire DTA as the sum of pure components. Therefore, it is advisable to have some idea about the origin of the unknown sample. This limitation of DTA suggests that in itself alone it is not a good identification technique and that the results should be interpreted in conjunction with other techniques such as X-ray diffraction, or spectroscopic analysis.

Roberts and Austin in 1899, suggested the use of reference inert material to measure the differential temperature. This method was first tested on different metals. Fenner first applied this technique for the identification of clay and silica minerals in United States, and it soon became the standard technique for the identification of different clays. Hollings and Cobb [1915] introduced the control of gaseous atmosphere around the sample.

Norton conducted studies on the identification of different clays by using DTA. He found a relationship in the area of the endotherm vs. the weight percent of a mineral in the mixture indicating proportionality between the weight of the mineral and the heat effect [Norton, 1939].

Wittels [1951] used DTA as a microcalorimeter. He found that the area of the endotherm is linearly related to the heat of reaction for given mass of the sample. Around the same time, Berger and Whitehead [1951] used DTA to investigate the coalification process. In their study they find that lignin gives an exothermic peak between $400-500^{\circ} \mathrm{C}$ while cellulose yields an endothermic peak at $400^{\circ} \mathrm{C}$. Measurements on a variety of samples show that the lignin peak is identifiable in bituminous coals and lignites. The peak is absent for anthracites, indicating the complete conversion of the lignin type structures to thermally more stable forms at the end of coalification. Indications for small amounts of cellulosic structures were found in peat and lignite but they were absent in bituminous coals. Based upon this lignin theory a coal formation was reemphasized.

An extensive investigation of the relationship between coal rank and the characteristics of DTA curve was made by Glass [1955]. The DTA curves are classified into five distinct types: a) the meta anthracite type, characterized by single endothermic peak at 725-735 C; b) the anthracite type showing a single volatile loss peak at $630-680^{\circ} \mathrm{C}$; c) the low volatile bituminous type endotherm at $500-620^{\circ} \mathrm{C}$; d) the high volatile bituminous type where primary volatilization endothermic effect is divided into two sharp exothermic reactions; and e) subbituminous type having a large primary volatilization peak at $450^{\circ} \mathrm{C}$.

Garn and Flaschen [1957] report the use of DTA for the analysis of impurities in mineral matter. Schwenker and Beck [1960] presented a study on the detection of transition temperatures for textile fibers.

Jen Chiu has shown that DTA can be used to identify the organic compounds [Chiu, 1962]. Sanderman and Augustine [1963], applied DTA to determine the thermal stability for different woody components. The order they rank is lignin $>$ cellulose $>$ hemicellulose. Hemicellulose decomposed exothermically at about $200^{\circ} \mathrm{C}$ in nitrogen. However, some polysaccharides would decompose closer to cellulose decomposition temperature. The thermal stability of poly and mono saccharides is reduced by the presence of carboxyl groups. Cellulose decomposes with an endotherm commencing around $290^{\circ} \mathrm{C}$. Sometimes an exotherm is seen at around $350^{\circ} \mathrm{C}$. The lignin has a flat exotherm peak commencing at $300^{\circ} \mathrm{C}$ and reaching a maximum at $425^{\circ} \mathrm{C}$. 
DTA can easily detect the stability ranges for a sample. One of the major advantages is that the results can be obtained with much rapidity.

DTA has proven to be of great value for the analysis of metastable and unstable systems. A metastable system has a higher free energy than the corresponding equilibrium system, but does not change noticeably with time. An unstable system in contrast is in the process of changing towards the equilibrium and can only be analyzed as a transient state. Metastable states usually become unstable during DTA which operates without temperature gradients in the sample and reference. If a sizable temperature gradient exists within the sample the sensitivity may be higher than with smaller gradients because one can work with larger masses, but for quantitative heat measurements it is desirable to have as little temperature gradient in the sample as possible. Samples such as some oxides and organic materials have lower thermal conductivity and therefore may have internal temperature gradients. Detailed history of DTA development and its applications is given by Mackenzie [1970]

\subsection{ICTA recommendations}

In 1965 the First International Conference on Thermal Analysis [ICTA] established a committee on standardization charged with the task of studying how and where standardization might further the value of thermal analysis. One area of concern was for the uniform reporting of data, in view of the profound lack of essential experimental information occurring in much of the thermal analysis literature. Because thermal analysis usually involves dynamic techniques, it is essential that all pertinent experimental details accompany the actual experimental records to allow their critical assessment. This was emphasized by Newkirk and Simmons [Wunderlich, 1990], who offered some suggestions for the information required with the curves obtained by thermogravimetry.

The actual format for communicating these details will of course depend on a combination of the authors' preference, the purpose for which the experiments are reported and the policy of the particular publishing medium. To accompany each TG or DTA record, the following information should be prepared:

1. Identification of all substances (sample, reference, diluent) by a definitive name, empirical formula or an equivalent compositional data list

2. A statement of the source of all substances, details of their histories, pretreatments, and chemical purities, so far as these are known.

3. Measurement of average rate of linear temperature change over the temperature range involving the phenomena of interest.

4. Identification of the sample atmosphere by pressure, composition, and purity whether the atmosphere is static self-generated, or dynamic through or over the sample. Where applicable the ambient atmospheric pressure and humidity should be specified. If the pressure is other than atmospheric, full details of the method of control should be given.

5. A statement of the dimensions, geometry and materials of the sample holder; the method of loading the sample holder; the method of loading the sample where applicable. 
6. Identification of the abscissa scale in terms of time or of temperature at a specified location. Time or temperature should be plotted to increase from left to right.

7. A statement of the methods used to identify intermediates or final products.

8. Faithful reproduction of the original records.

9. Wherever possible, each thermal effect should be identified and supplementary supporting evidence stated.

In the reporting of TG data, the following additional details are also necessary.

10. Identification of the thermobalance, including the location of the temperature measuring thermocouple.

11. A statement of the sample weight and weight scale for the ordinate. Weight loss should be plotted as a downward trend and deviations from this practice should be clearly marked. Additional scales (e.g. fractional decomposition, molecular composition) may be used for the ordinate where desired.

12. If derivative thermogravimetry is employed, the method of obtaining the derivative should be indicated and the units of the ordinate specified.

In reporting DTA traces, these specific details should be presented:

13. Sample weight and dilution of the sample.

14. Identification of the apparatus, including the geometry and materials of the thermocouples and the locations of the differential and temperature measuring thermocouples.

15. The ordinate scale should indicate deflection per degree centigrade at a specified temperature. Preferred plotting will indicate upward deflection as a positive temperature differential, and downward deflection as a negative temperature differential, with respect to the reference. Deviations from this practice should be clearly marked. We have tried to follow these recommendations in this Atlas.

\section{7 . Manufacturers}

Commercial DTA first became available in 1952 from the Robert L. Stone company. Stone's was the first quantitative application of DTA to a chemical system in the study of the polymorphism of $\mathrm{Na}_{2} \mathrm{SO}_{4}$ by Kracek in 1929. In this study temperatures for five different phase transformations were identified. Stone determined the heat of dissociation of magnesite to be $10.1 \mathrm{kcal} / \mathrm{mole}$. The thermogram showed increasing decomposition temperature with increasing $\mathrm{CO}_{2}$ pressure. When log $\mathrm{p}$ is plotted against $1 / \mathrm{T}$, the slope of the plot is $\Delta H / R$ and can be used to determine the heat of reaction from the Clausis-Clapyron equation, i.e.

$$
d(\ln P) / d(I / T)=-Z(\Delta H / R)
$$


Where, $Z$ is the compressibility factor, $P$ is the pressure, $\Delta H$ is the heat of reaction, $T$ is the temperature and $\mathrm{R}$ is the gas constant.

There are several commercial manufactures of TG equipments. Prominent among those are Perkin-Elmer, Du Pont, Stanton Redcroft, Seiko Instruments, Cahn, Sinku Rico, and Mettler. The differences in the design of various equipment in terms of accuracy for parameter measurements is mainly in the placement of the thermocouple for the measurement of sample temperature or the mechanism of gas solid contact, i.e. whether the gas flows over the sample, through the sample or diffuses into the sample. Difference can also be seen in terms of sample holder to sample size ratio. This aspects relates to the temperature deviation within the sample at given heating rates.

The operating limits on these models also varies. Instead of discussing each model we present the extreme limits available. The maximum sample size that can be subjected to thermal decomposition is $10 \mathrm{~g}$ in the Cahn TG-131 model. (This thermobalance can hold a sample size up to $100 \mathrm{~g}$ but the dynamic range is only $10 \mathrm{~g}$ ). All the other models restrict maximum weight up to $200 \mathrm{mg}$. The maximum heating rate of $100^{\circ} \mathrm{C} / \mathrm{min}$. is available in almost all of the commercial models. However, the slowest heating rate of $0.1^{\circ} \mathrm{C} / \mathrm{min}$. is available in the Seiko SSC 5200 model. A pressure variation from 5 torr to ambient is available in most of the commercial designs. Only the Cahn TG-151 has the capability to go up to 1000 psig. The determination of temperature calibration by using Curie point method is easiest in PerkinElmer model TG-7.

The data presented in this book were collected on a Seiko Instruments TG/DTA SSC 5200 model. One of the major advantages of this unit is that it can collect data on the same sample simultaneously for TG and DTA. This feature is not present in most other commercially available units. We will discuss the details of this instrument and the procedure for data collection in this Atlas in chapter 11. Some other models can record data for TG and DTA simultaneously (Cahn TG-131/DTA-131) but they require a different sample for TG and DTA recordings. 


\section{Chapter 10}

\section{DERIVING KINETIC DATA FROM THERMAL ANALYSIS}

\subsection{Introduction}

The determination of kinetics of decomposition for biomass and other fuels from thermal analysis data have long been a source of fascination to those working in this field. It is projected that an accurate determination of the kinetics for a particular substance would be useful in designing plants and processes for that substance. To the best of our knowledge the existing kinetic knowledge has never been applied because there was such a scatter in kinetic data that no one knew which data ato use.

It is a relatively simple matter to fit TG curves with an equation of the form [Milne, 1981]

$$
d V / d t=k V^{n}
$$

where

$k=A \exp (-E / R T)$ and $V$ is the fraction of total volatiles remaining at temperature $T$ and $n$ is the order of reaction. The exponential term is sometimes interpreted as an activation energy; $A$ is the pre-exponential factor and $R$ is the gas constant.

If the sample is heated at a constant rate, $a=d T / d t$, then this equation becomes

$$
d V / d T=k V / a
$$

Because of its importance and its relatively fixed structure, a number of workers have used cellulose to determine the decomposition kinetics and decomposition mechanisms [Friedman, 1965; Chatterjee, 1965; Broido, 1969; Shafizadeh, 1979; Diebold, 1985 and Agrawal, 1986]. In particular Antal has written two extensive reviews on the decomposition kinetics of cellulose and lignocellulosics based on various proposed reaction schemes involving up to five steps [Antal, 1982;1985]. More recently Varhegyi, Jakab and Antal have published another review which suggests that a single step is sufficient for describing the kinetics of the cellulose decomposition [Antal, 1994; Varhyegi, 1994].

Cellulose comes close to being an identifiable "biomass compound" with potentially reproducible kinetics. Yet the kinetic exponential factor $E$ (sometimes interpreted as an activation energy) has been reported to vary from 50 to $250 \mathrm{~kJ} / \mathrm{mole}$ as shown in Fig. 10-1, which shows an excellent correlation between the. This has been called the "compensation effect" [Chornet, 1979, Reed, 1985]. Much of this variation is illusory; TG data plotted from any of the pairs of $A$ and $E$ in Fig. 10-1 would show cellulose breaking down at temperatures between $280^{\circ} \mathrm{C}$ and $400^{\circ} \mathrm{C}$ with varying slopes in between. Much of the design work on cellulose would not need to be more accurate that this.

The variation in the kinetic factors can be attributed to variation in sample size and heating rate (discussed in Chapter 8 ), variations in the methods used for mathematical analysis (Chapter 10), and instrumental errors coupled with sample impurities (Chapter 11). 
Recently there has been some progress in determining the sources of the discrepencies and deriving reliable data [Antal, 1994; Fritsky, 1994; Gaur, 1994;Varhegyi, 1994; Abatzoglou, 1992; Belkacemi, 1991]. We hope that the data in this Atlas can provide data for future kinetic analysis, lead to a better understanding of the kinetics of biomass thermal decomposition and also be used for practical design.

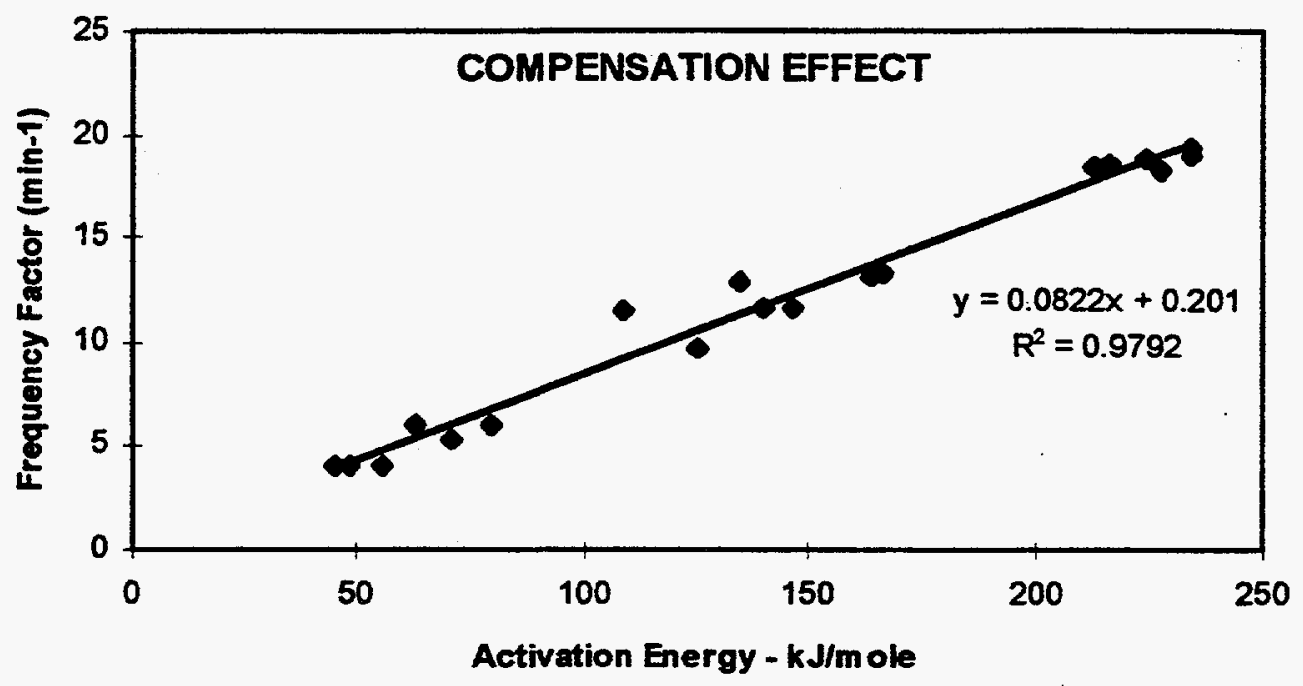

Fig. 10-1 Compensation effect for Cellulose Kinetics, showing measured values of the kinetic factors Log $A$ and $E$ taken from the literature [Chornet 1979, Reed, 1985]

For those wishing to do kinetic analysis, we have tried to put together some of the different methods available in thermal analysis literature (TG and DTA) for the interpretation of data to determine the kinetic parameters. We are however, not attempting to rank these methods because we believe that the reader of this book can make the judgment as to which method will satisfy their needs in the best way.

10. 2 Determination of kinetic parameters using thermogravometric data 10.2.1 Coats and Redfern method:

For a reaction $A_{(s)} \rightarrow B_{(s)}+C_{(g)}$ the rate of conversion of $A$ can be given as

$$
d X / d t=k(1-X)^{n}
$$

where $\mathrm{X}$ is the fractional conversion and $\mathrm{k}$ is the rate constant expressed as

$$
k=A \exp (-E / R T)
$$

$A$ and $E$ are Arrhenius parameters known as frequency factor and the activation energy respectively, while $n$ is the reaction order [Coats, 1964]. Since not much theoretical justification can be provided for frequency factor and reaction order in the case of solid decomposition, Gadalla recommends the use of terms "pre-exponential factor" and 
"exponent term" respectively, to avoid any misconception. In this book we have tried to adhere to this terminology [Gadalla, 1985].

For a linear heating rate, $a=d T / d t$, the substitution for $k$ in equation 1 can be made as follows

or

$$
\begin{aligned}
& d X d T=A a \exp (-E / R T)(1-X)^{n} \\
& d X /(1-X)^{n}=A V \exp (-E / R T) d T
\end{aligned}
$$

In order to integrate equation (10.4) within limits of $X=0$ to $X$ and $T=0$ to $T$, at first substitute $u=E / R T$. The approximate solution for the right hand side of this integral is then given as

$$
\int e^{(-u)} u^{(-b)} d u=u^{(1-b)} e^{-u} \Sigma(-1)^{n}(b)^{n} /\left(u^{(n+1)}\right)
$$

Substituting this solution into equation 4 for $n$ not equal to 1 gives

$$
\left(1-(1-X)^{(1-n)} /(1-n)=A R T^{2} / a E[1-2 R T / E] e^{-E R T}\right.
$$

and for $n=1$ equation (10.4) becomes

$$
-\log \left[(1-X) / T^{2}\right]=A R / a E[1-2 R T / E] e^{-E R T}
$$

To determine the kinetic parameters for any reaction by using this method one has to make a guess at the reaction order. Subsequently, by taking log of both sides, equations 10.6 \& 10.7 transform into the linear function $y=m x+c$. The reaction order for which the linearity of the function is best defined can then be used to determine the kinetic parameters by using the graphical technique.

This method of Coats and Redfern [Coats, 1964], has been used successfully by many researchers. However, one of the drawbacks of this method is that the reaction order has to be known beforehand or one has to do extensive linear fit on the experimental data for various reaction orders. Another limitation is that for cases where there is step wise conversion, such as for calcium oxalate or polyvinyl chloride, the treatment of the data has to be done for separate steps individually.

\subsubsection{Gyulai and Greenhow method:}

This method considers decomposition of solids at two heating rates for the prediction of the kinetic parameters. By doing so the authors have eliminated the requirement of assuming the rate function or the reaction order as is the case with the Coats and Redfern method.

The basic rate equation used by Gyulai and Greenhow [Gyulai, 1974] is similar to equation (10.1) except that they do not define the form of the solid conversion function.

$$
\int d X d T=\int A / a \exp (-E R T) f(X)
$$


In this case the solution is even more complicated because if we separate the variables

$$
\int d X f(X)=\int A V a \exp (-E / R T) d T
$$

then the unknown term is present on both sides of the equation; the right hand side of the expression does not have an exact integral solution and on the left, the form of the function for the conversion of the solid is not known.

By substituting $F(X)=\int[d(X) / f(X)]$ in equation (10.9) we obtain

$$
F(X)=A v \int^{*}\left[\exp (-E R T) d T^{-}\right.
$$

If $i=\int[\exp (-E / R T) d T]$ then

$$
F(X)=(A \vee a)(i)
$$

For two points corresponding to two identical conversion extents $X$, on two TG curves obtained at two different heating rates $a_{1}$ and $a_{2}$ for the same sample and initial weight we can write

$$
\begin{aligned}
& i_{11}=\int[\exp (-E / R T) d T] \\
& \dot{i}_{12}=\int[\exp (-E / R T) d T]
\end{aligned}
$$

and

$$
\begin{aligned}
& F(X)_{11}=\left(A a_{1}\right)(i) \\
& F(X)_{12}=\left(A a_{2}\right)(i)
\end{aligned}
$$

where the first figure on the subscript relates to the conversion extent and the second value for the TG curve.

Since the points have been obtained for the same conversion level and the sample is the same, they would have in all probability exhibit the same conversion function and hence

$$
F(X)_{11}=F(X)_{12}
$$

this follows

or

$$
\begin{aligned}
& F(X)_{11} / F(X)_{12}=\left(a_{2} / a_{1}\right) /\left(i_{11} / i_{12}\right)=1 \\
& a_{1} / a_{2}=i_{11} / i_{12}=\ldots=i_{n j}=i_{n k}
\end{aligned}
$$

For the determination of the activation energy, two points of the same conversion are chosen on two TG curves obtained at two different heating rates and the corresponding temperatures $T_{11}$ and $T_{12}$ are noted. The value of $\log i$ corresponding to these temperatures at various activation energies can be obtained from the tabulated values given by authors, or from any of the approximate solutions such as the one given by Coats and Redfern. The plot of $\log \left(i_{1} / i_{2}\right)$ against $E$, would help in finding that value of $E$ corresponding to which $\log \left(a_{1} / a_{2}\right)$ $=\log \left(i_{1} / i_{2}\right)$. 
Now for the two points on same TG curve we can write

$$
F\left(X_{1}\right)=(A / a) i\left(E_{1}, T_{1}\right) ; F\left(X_{2}\right)=(A / a) i\left(E_{2}, T_{2}\right)
$$

Then depending on the type of conversion function, say $F(X)=\int d X(1-X)^{n}$ we want to explore, the integral solution of the function can be obtained, for example in this case for $n$ not equal to 1

$$
\left.F(X)=(1 /(n-1))\left(1 /(1-X)^{n-1}\right)-1\right)
$$

and for $n=1$

$$
F(X)=-\ln (1-X)
$$

Now we choose one reference conversion say $X_{2}$ and calculate the ratio $\log \left(F\left(X_{1}\right) / F\left(X_{2}\right)\right)$ for a range of values from the conversion $X$ data. This ratio is then plotted against $n$ using equation (10.20). The order of the reaction for a conversion $X_{1}$ is obtained from the graph as the value of $n$ corresponding to $X=X_{1}$ and $\log \left(F\left(X_{1}\right) / F\left(X_{2}\right)=\log \left(i\left(E_{1}, T_{1}\right) / i\left(E_{2}, T_{2}\right)\right)\right.$. After these determinations the pre-exponential factor is calculated with the help of equation (10.11).

\subsubsection{Doyle's method:}

Doyle's method [Doyle, 1961] deals with the decomposition in each step as being independent. The apparent rate of volatilization is found by

$$
d V / d t=-a d W / d T
$$

where, $W$ is the initial weight, $a$ is the heating rate and $V$ is the volatile portion of the solid. However, if there are different volatilization steps, then for a particular step the appropriate residual mass fraction $h$, is calculated on the total fraction volatilized during the step, rather than on the total initial mass

$$
h=(W-G) / H
$$

where, $\mathrm{H}$ is the total mass fraction volatilized during the step and $\mathrm{G}$ is the weight fraction remaining after the step has been completed.

Hence, now we can write

$$
d V / d t=H(d h / d t)
$$

We can define dh/dt with a kinetic expression

$$
-d h / d t=k f(h)
$$


where $k=A \exp (-E / R T)$ and $f(h)$ is the function for solid conversion. The conversion of the solid for a given step has been written by Doyle in the same manner as by others

$$
\int d h / f(h)=A v a[\exp (-E / R T) d T]
$$

same, they would in all probability exhibit the same conversion function and hence

$$
\int d h / f(h)=A V a \int[\exp (-E R T) d T]
$$

The solution of the right hand side of the equation is done by substituting $u=E / R T$ and then obtaining the approximation $p(x)$ for the above integral.

$$
\int d h / f(h)=g(x)=(A / a)(E / R) p(x)
$$

where

$$
\log p(x)=-2.315-0.4567 E / R T
$$

Doyle points out that the value of $\mathrm{Aa}$ can be evaluated on the basis of a single thermogram slope $\mathrm{dV} / \mathrm{dT}$ and the corresponding absolute temperature $\mathrm{T}$.

$$
A / a=(\exp (-E / R T) / H f(h))(d V / d T)
$$

The value of $E$ is evaluated by first determining $X_{a}$ the value of $X$ at the corresponding value of $T_{a}$

$$
E=\left[\left(R H f\left(h_{a}\right) g\left(h_{a}\right) \exp \left(-X_{a}\right)\right) /\left(p\left(x_{a}\right)\right](d T / d V)_{a}\right.
$$

Doyle's method was one of the earliest methods developed for the determination of the kinetic parameters using non-isothermal TG data.

\subsubsection{Zsako method:}

Zsako [Zsako, 1970; 1973] has considered a more general form of rate expression then the methods discussed up to now in this chapter.

$$
d X / d t=k X^{a}(1-X)^{b}
$$

where $a$ and $b$ are empirical constants.

In this method Zsako has tried to provide an improvement in terms of applying Doyle's method for reactions which have more complex conversion functions $f(X)$. Taking log of equation (10.27) gives

$$
\log [(A \vee a)(E / R)]=B=\log g(X)-\log p(X)
$$

Now once again taking log of the left side of equation (10.32)

$$
\log A=B+\log (R a)-\log E
$$


Zsako has determined the analytical forms of conversion function $(1-X)^{b}$ and term $B$ for reaction orders (b) eg; $0,1 / 3,1 / 2,2 / 3,1$ and 2 . In this method the function with most constancy over the entire thermogram can be considered as the conversion function of the solid. The value of $p(x)$ is given by Doyle as a function of activation energy.

\subsubsection{Satava and Skvara method:}

The method of Satava and Skvara [Satava, 1969] is basically a more general form of the method proposed by Zsako. The rate expression used to describe the solid decomposition by Zsako is given as equation (10.31).

At constant heating rate the decomposition of solid can be written as equation (10.9). The integral of the left side of this equation can be performed once the decomposition function for the solid is known. In this method since generalized forms of the function have been considered, no single solution is available. The right hand side has been integrated by Doyle and many others. The authors of this method preferred to use the solution provided by Doyle (equation 10.27). Taking logarithms of both sides of equation (10.27) gives equation (10.32). It can be seen that quantity $B$ is independent of temperature.

Satava and Skvara determined the values of $\log g(X)$ for various functions. The major advantage of this method lies with the fact that the authors have considered and provided solutions to the different forms of conversion functions.

Now, since both the terms on the right hand side of equation (10.32) are known, the values for B can be determined. Satava and Skvara propose a graphical technique for the determination of value for $B$. The values of $X$ at intervals of 0.05 and the corresponding temperature $T_{X}$ are first read from the $T G$ curve. The $\log g(x)$ values for the various rate processes are plotted against the corresponding $T_{x}$ values on a transparent paper. On the same scale a plot of $-\log p(x)$ versus $T$ is also drawn. The plot of $\log g(x)$ is placed on top of the $\log p(x)$ diagram so that the temperature scales coincide and is then shifted along the ordinate until one of the $\log g(x)$ curves fits one of the $\log p(x)$ curves. From this $\log p(x)$ curve, the activation energy $E$, is determined. The $\log g(x)$ curve which fits the $\log p(x)$ curve is the most probable kinetic function describing the thermal decomposition of that particular solid. When the two plots are placed so that the values on the axes coincide, the distance between the $\log g(x)$ and $\log p(x)$ curves is the value for $B$ which is used to quantify preexponential factor $\mathrm{A}$ by using equation (10.31).

It is, however, advised that in order to be fully confident of the conversion mechanism determined by this method one should also check it with some other method apart from TG analysis to rule out the possibility of mathematical coincidence of curve fitting.

\subsubsection{Freeman and Carroll method:}

This method helps in determining the kinetics of a reaction over the entire range of temperature [Freeman, 1958]. The kinetic parameters are determined by using equation $\left(10.34^{\prime}\right.$

$$
\Delta \circ \log (\mathrm{a} C / d t) / \Delta \log (1-C)=n-(E / 2.3 R)(\Delta(1 / T)) /(\Delta \log (1-C))
$$

This method is also called a difference differential method. A plot of $\Delta \log (a \mathrm{dC} / \mathrm{dt}) / \Delta \log (1-$ C) versus $\Delta(1 / T) /(\Delta \log (1-C))$ is used to determine the value for activation energy and then 
gives the reaction order. It has been found that by the use of this equation the results are in good agreement at low conversion levels.

\subsubsection{Ingraham and Marier method:}

Ingraham and Marier developed this method for reactions which follow linear kinetics for the decomposition such as calcium carbonate. The rate expression for such a reaction can be written as

$$
d w / d t=k
$$

where $d w$ is the mass loss per unit area in time $d t$. If the temperature of the sample is increased at a linear heating rate the temperature at any given time can be given as

$$
T=b+a t
$$

where $b$ is the initial temperature and $a$ is the heating rate. These authors developed equation (37) for the determination of activation energy

$$
\log (d w / d T)=\log T-\log a+\log C-(E / 2.3 R)
$$

The activation energy is calculated from the slope of a plot of $[\log (d w / d T)-\log T+\log$ a] versus $1 / T$. The $\log (a)$ value permits the correction of TG curves obtained at different heating rates. We will discuss the correction of TG curves at different heating rates in much detail later in this chapter.

\subsubsection{Vachuska and Voboril method:}

This is a differential method for the determination of kinetic parameters using thermogravimetric data.

$$
\begin{array}{ll}
d X / d t=k(1-X)^{n} \exp (-E / R T) \\
\text { or } & \ln (d X / d t)=\ln k+n \ln (1-X)-E R T
\end{array}
$$

Since $X$ and $T$ are function of time in the case of TG data, we can differentiate the above equation with respect to time

$$
\left(d^{2} X / d t^{2}\right) /(d X / d t)=-(n / 1-X)(d X / d t)+\left(E / R T^{2}\right)(d T / d t) \quad \text { (eq. 10.40) }
$$

rearrangement of equation (10.40) gives

$$
\left(d^{2} X / d t^{2}\right) T^{2} /(d X / d t)(d T / d t)=-n(d X / d t)\left(T^{2}\right) /[(1-X)(d T / d t)+E / R \text { (eq. 10.41) }
$$

This equation can be used to determine reaction order and activation energy. 
10.2.9 Varhegyi's Integral solution:

Varhegyi [Varhegyi,1978] has provided the integral of the rate expression considering the temperature dependency of the pre-exponential factor. He considered the rate expression of the form

$$
k=A T^{b} \exp (-E R T)
$$

and then provided the integral of equation (10.42) with respect to temperature

$$
\int k d T=\int A T^{b} \exp (\cdot E R T) d T
$$

Substituting $y=E / R T$ and $b=s-2$ in equation (10.43)

$$
\int T^{b} \exp (-E R T) d T=p_{s}(y)=(E R)^{(s-1)} \int y^{-s} \exp (-y) d y
$$

The solution of the integral is provided by using continued fractions due to Legendre

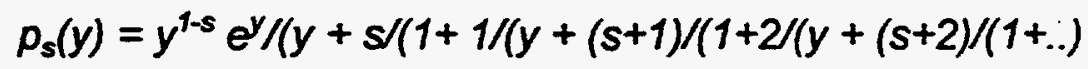

If equation (10.45) is truncated at the sixth sign of division, the relative error of the approximation is about $10^{-5}$ at $y$ values of 10 . In thermal analysis the values of $y$ are not less than 10.

Another solution to $p_{s}(y)$ integral with relative error being of the same order of magnitude as in equation (10.45) is

$$
p_{s}(y)=y^{-s} e^{-y}\left(1+a_{1} /(y+1)+a_{2} /(y+1)(y+2)++a_{n}(y+1)(y+2) . .(y+n)\right)
$$

This series was proposed by Schlomlich [Van Krevelen, 1951]. The method for the determination of coefficents $a_{1} . . a_{n}$ is given by Bateman and Erdelyi [Bateman, 1953]. Varhegyi also lists Pede's approximation [Luke, 1969]

$$
p s(y)=y^{s} e^{y}(y+1) /(y+s+1)
$$

The rate equation for decomposition can be written as

$$
\int d X / f(X)=g(X)=1 / a \int(k(T) d T)
$$

Using equation (10.44), equation (10.48) can be written as

$$
g(X)=A / a(E / R)^{s-1} p_{s}(y)
$$


but $p_{s}(y)$ is a product of $y^{-s} e^{-y} q_{s}(y)$, where $q_{s}(y)$ is the approximation for last term in equation (10.47). Substituting this value for $p_{s}(y)$ we can write

$$
g(X)=(A R / a E) T^{s} e^{-y} q_{s}(y)
$$

or

$$
\ln g(X) / T^{s}=\ln (A R / a E)+\ln q_{s}-y
$$

The term $\ln \left(q_{s}\right)$ can be approximated by expansion using Taylor's series

$$
\ln \left(q_{\mathrm{s}}\right)=c_{0}+c_{1} y
$$

The values for $E$ and $A$ are evaluated in the following manner: At first the term $\ln (g(X) / T s)$ is obtained from the experimental data. Then these values are approximated by a linear function of $1 / \pi$

$$
\ln (g(X) / T s)=B_{0}+B_{1} 1 / T
$$

At the first approximation we can get an estimate of coefficient $B_{1}$ which is equal to $-E / R$. Then an average temperature $T$ is chosen somewhere in the middle of the temperature interval of decomposition and then using the first approximation for $E$ the corresponding value for $y=E / R T$ is determined. Subsequently using this value of $y$ the coefficients for the expansion of in $\left(q_{s}\right) c_{0}$ and $c_{1}$ are calculated.

$$
\begin{aligned}
& c_{1}=s /[(y+1)(y+s+1)] \\
& c_{0}=\ln [(y+1) /(y+s+1)]-c_{1} y
\end{aligned}
$$

Knowing the values of $c_{1}$ and $c_{0}$ will help in determining the more correct values for $E$ and $A$ by using equations (10.49-10.51) and rearranging them to get

$$
\begin{aligned}
& B_{1}=-\left(1-c_{1}\right) E / R \\
& B_{0}=\ln (A R / a E)+c_{0}
\end{aligned}
$$

The difference in the prediction of $E$ value or the activation energy by going through this method is only of the order of $3-5 \%$ but the value for $A$ is improved by a factor of $50 \%$.

Flynn and Wall and Satava and Skavara have also shown in their independent study that the linear dependency of pre-exponential factor on the temperature has no significant effect but the change in heating rate has a dramatic effect. They however do not combine this effect with the temperature dependency of A factor with the power law.

\subsubsection{Gaur and Reed method:}

Varhegyi's integral has shown that if the pre-exponential factor is not considered as temperature dependent with power function its estimation can be off by $40-50 \%$. Satava and Skavara [Satava, 1978] have shown in their independent study that the effect of heating rate and the change in pre-exponential term result in similar changes in the weight loss curve of a 
sample. Flynn and Wall [Flynn, 1966] have shown that there is negligible change on the pre-exponential factor if it is considered as linear temperature function but on the other hand they also state the heating rate makes a lateral shift on the curve.

Gaur and Reed [Gaur, 1993] have developed an equation which incorporates the change in pre-exponential factor as a function of heating rate.

The rate of decomposition can be given as

Where,

$$
\begin{gathered}
d X d t=A_{0} \exp \left(-E_{d} / R T\right) f(X)^{n} \\
A_{0}=k^{\prime} T m \\
E_{0}=E_{\text {exp }}-R T
\end{gathered}
$$

In the above equations parameters $k^{\prime}$ and $E_{0}$ are constants which are independent of temperature while $A_{0}$ and $E_{\exp }$ are temperature dependent. Equations (10.59) and (10.60) are the modified forms of the original Arrhenius derivation where the exponent $m$ has an explicit value of 0.5 . Since $A_{0}$ varies with temperature, the equality of the temperature independent term $\mathbf{k}$ at two different temperatures can be written as

$$
\begin{aligned}
& k^{\prime}=A_{\sigma} T^{m}=A_{0, i}=T_{i}^{m} \\
& A_{0, i}=A_{0}(T / T)^{m}
\end{aligned}
$$

where $T$ refers to the condition at which kinetic parameters are determined and $T_{i}$ refers to the temperature at which the predictions for solid decompositions are to be made.

Substitution of equation (10.62) in equation (10.58) at temperature $T_{i}$ gives

$$
d X d t=A_{0}(T / T)^{N m} \exp \left(E_{\delta} / R T\right) f(X)^{n}
$$

The temperature ratio in the above expression can be written in terms of heating rates $\left(a_{\mathrm{f}} / a\right)^{\wedge m}$, where, $a_{i}=d T_{j} / d t$ and $a=d T / d t$. Since rate constant for this reaction is close to zero at $T<T_{0}, T_{0}$ can be considered as zero degrees centigrade. For limits $T=0$ at $t=0$ and $T=T$ at $t=t$ and at equal time intervals

$$
a / a=T / T
$$

Substituting (10.64) in (10.63) gives;

$$
d X d t=A_{0}(a / a)^{m} \exp \left(-E_{\delta} R T_{i}\right) f(X)^{n}
$$

expressing the above rate expression for constant heating rate TG data we get;

$$
d X d T=A_{\sigma} / a_{i}\left(a / a_{i}\right) m \exp \left(-E_{d} / R T_{j}\right) f(X)^{n}
$$

The solution for this equation has been provided by the authors for $f(X)^{n}=(1-X)^{n}$ function using Doyle's approximation for the integration.

for $n=1$ 


$$
-\ln (1-X)=\left(E_{0} A_{d} / R a_{j}\right)\left(a / a_{j}\right)^{m} \text { 10(-2.315-0.4567Eo/RT) }
$$

and for $n \neq 1$

$$
[1-(1-X) 1-n] /(1-n)=\left(E_{0} A_{O} / R a_{j}\right)\left(a / a_{j}\right)^{m} 10(-2.315-0.4567 E \alpha / R T)
$$

\subsection{Determination of kinetic parameters using differential thermal analaysis data}

Several methods have been proposed for the determination of reaction kinetics by using a differential thermal analysis (DTA) curve. Some of the commonly used methods are the Kissinger method, the Tateno method, and the method of Borchardt and Daniels [Borchardt, $1956 ; 1957]$. In the Kissinger method the variation in peak temperature with heating rate has been used to determine the activation energy [Kissinger, 1957]. The Tateno method is described by the means of transfer functions and gives values for reaction order and activation energy [Tateno, 1966].

In this book we present the method of Bocrhardt and Daniels which is the most extensively used method. This method was developed for homogeneous reactions.

The application of DTA studies to determine the kinetic parameters was successfully attempted by Borchardt in his Ph.D work at the university of Wisconsin, [1956]. In his work he determined the reaction kinetics by using only one non-isothermal curve. Based upon this work Borchardt and Daniels [1957] developed the method for the determination of reaction kinetics for homogeneous solutions. This method employs the development of a heat balance equation around the sample.

$$
C_{p} d T=d H+k \Delta T d t
$$

where $\mathrm{dH}=$ heat of reaction, $\mathrm{C}_{p} \mathrm{dT}=$ enthalpy of the reaction solution, and $\mathrm{k} \Delta \mathrm{T} \mathrm{dt}=$ the heat transferred into the cell from the surroundings.

$$
d H=C_{p} d T-k \Delta T d t
$$

If $C p$ and $k$ are independent of temperature then for $t_{0}$ to $t_{\infty}$

$$
\Delta H=C_{p}\left(\Delta T_{\infty}-\Delta T_{0}\right)+k j \Delta T d t
$$

at $T=\infty \Delta_{\infty} \Delta \Delta T$ is the temperature difference between the sample and the furnace. Therefore,

$$
\Delta H=k A
$$

where $A$ is the total area under the curve. Since $d H$ is the heat transferred due to reaction on per mole basis, $-k A n_{0}$, we can write the following expression for fractional conversion in homogeneous reaction as

or

$$
d H=-k A n_{0}(d n)
$$

Substituting (10.73) in (10.69) gives 


$$
-d n / d t=\left(n_{\sigma} / k A\right)\left[C_{p}(d T / d t)+k \Delta T\right]
$$

where, number of moles present at any instant are given as

$$
n=n_{0}-\int d n / d t
$$

The development of the kinetics method for heterogeneous systems was proposed by Blumberg [Blumberg,1959]. This method is an extension of the method presented by Brochardt and Daniels for the homogeneous reactions. However, this extension has been subject to lot of criticism due to its assumption of negligible temperature gradient within the solid sample. In addition, the relationship of the peak area in a thermogram to the heat of reaction has also been reported by Ramachandran and Bhattacharya [Ramachandran, 1954]

\subsubsection{Other effects in understanding DTA data}

During dynamic heating there is a chance of temperature lag increase with increasing heating rate. The slower the rate of heating, the smaller the variation. However, with a slow rate of heating, the temperature differential between the reference material and the substance examined will be less. This results in rounded peaks that tend to be more significant as the heating rate is lowered. On the other hand with a rapid heating rate the peak becomes intense but a lot of details are lost. The theory behind this phenomenon is explained as follows: The temperature at any point lags behind that of the holder by an amount which depends only on the position of the point. This quasi steady state is reached after a time $\tau$ and for a cylindrical sample of radius and length $4 r$.

$$
\tau=1.08 \rho \mathrm{Cr}^{2} / \lambda
$$

Changes in thermal conductivity of the sample may result from temperature variations and from changes in composition produced by the reaction. The heating rate has an effect on peak height and peak width. A slow heating rate requires a sensitive recording system while with fast heating rate the neighboring peaks tend to coalesce. The optimum heating rate depends on the nature and characteristics of the sample and reference material. The International Geological Congress, London, recommends a heating rate of $10^{\circ} \mathrm{C} / \mathrm{min}$. Under these conditions peaks are of satisfactory size, overlap of neighboring peaks is not excessive and the time for determination is reasonable. The thermal effects in the sample are not uniform during the period of reaction. These can be reduced by using small samples and slow heating rates. The effect of particle size has been considered by several investigators. Norton has pointed out that finer particles give up their heat more rapidly [Norton, 1939]. 


\section{Chapter 11}

\section{EXPERIMENTAL APPARATUS AND DATA COLLECTION}

\subsection{Seiko TG/DTA}

The data reported in this Atlas were collected using a Seiko SSC 5200 TG/DTA thermal analysis apparatus. The schematic of the instrument is shown in figure 11.1. This apparatus has two horizontal balance arms containing thermocouples to support the pans and the sample which allows for close measurement of the sample temperature with negligible temperature deviation over the entire heating range of the furnace. The two balance arms permit taking simultaneous TG/DTA data on the same sample and minimizes the errors in analysis caused by the sample heterogeneity. One balance arm is used to support the reference inert material and the other arm supports the sample under study. The relative weight change between the sample pans as a function of temperature at a given heating rate give data for thermogravimetric analysis and the change in energy requirements is measured in terms of micro volts to provide data for differential thermal analysis.

The gas flows parallel to the balance arm and across the sample pan instead of parallel to the sample pan as is the case with most other models. This arrangement minimizes buoyancy effects caused due to change in gas density with change in temperature. We found that the deviation in the sample weight from ambient to $1100^{\circ} \mathrm{C}$ was about $30 \mu \mathrm{g}$. In our experience this is fairly negligible in comparison to most other thermal balances. The maximum permissible sample weight limit is $200 \mathrm{mg}$.

This TG/DTA model permits introduction of two reaction gases in any proportion at a flow rate of 100 to $600 \mathrm{cc} / \mathrm{min}$. The switching of gases is done with the help of gas flow controller which is regulated by the software provided along with the unit. In our opinion this software is user friendly and versatile.

One of the drawbacks of the system is that it is not MS-DOS compatible and has its own operating system. This slows the transfer of data to other MS-DOS compatible programs for further analysis such as for the determination of kinetic parameters. This problem was overcome by digitizing the data or transferring it by using communication software such as PROLOGIC.

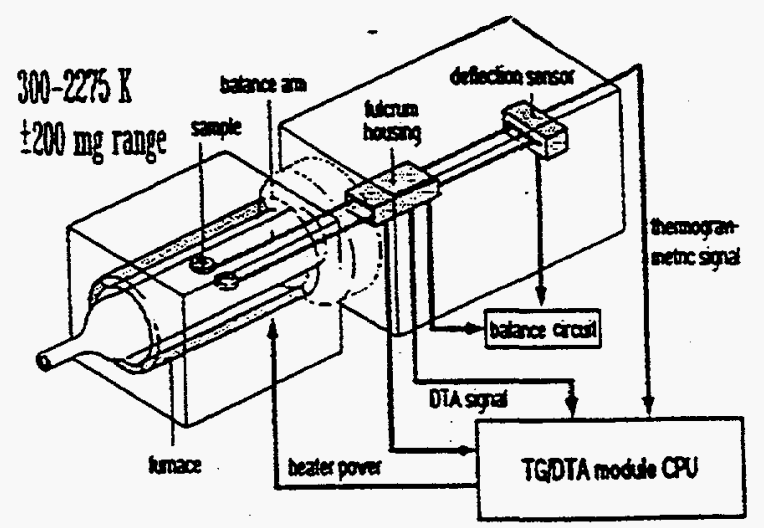

Figure 11.1 Schematic of Seiko SSC 5200 TG/DTA 


\subsection{Procedure for data collection}

The samples were first powdered by milling or grinding and heated to $120^{\circ} \mathrm{C}$ at $10^{\circ} \mathrm{C} / \mathrm{min}$. and were held there isothermally to allow for the removal of physical moisture.

Subsequently, the sample temperature was raised @ $10^{\circ} \mathrm{C} / \mathrm{min}$. to $950^{\circ} \mathrm{C}$ in the nitrogen atmosphere at a flow rate of $300 \mathrm{cc} / \mathrm{min}$. The net weight loss between $120^{\circ}$ and $950^{\circ} \mathrm{C}$ was recorded as total volatile matter. The residual char was then burnt in air at $950^{\circ} \mathrm{C}$ until a constant weight was obtained. The weight loss due to this combustion is reported as fixed carbon. The weight remaining is the ash content of the sample. The volatile portion of the fuel has been divided into two parts. The first part includes volatiles that are liberated between $120^{\circ}$ and $650^{\circ} \mathrm{C}$. This accounts for the major portion of the volatile fraction and is responsible largely for flaming combustion. This volatile fraction is sometime referred to as primary volatiles. The second portion of the volatiles sometimes termed to as secondary volatiles is liberated between $650^{\circ}$ and $900^{\circ} \mathrm{C}$. This volatile fraction takes little part in flaming pyrolysis and do not inhibit surface combustion. Unless stated otherwise all of the analysis was carried out with samples having particle size in the range of +10 to -100 mesh. A typical TG curve obtained from Seiko TG/DTA unit is shown as Figure 1 in the introduction. This data was used to generate the curves presented in the Atlas.

\subsection{Calibration of TG/DTA}

Thermal analysis is not an absolute measuring technique and hence calibration is of prime importance. The calibration of the sample temperature to the thermocouple reading was performed by determining the Curie point for some Ferro magnetic samples in the temperature range of $250-800^{\circ} \mathrm{C}$. In this procedure a magnet was installed just outside the furnace but close to the sample pan supports. Subsequently, the Ferro magnetic samples whose curie point temperature ( the temperature at which these samples loose their magnetic property) was known were placed in the sample pan. The TG/DTA furnace was then heated from ambient to $900^{\circ} \mathrm{C}$ at designated heating rate. The temperature at which these samples loose their magnetism is signified by the weight change due to the absence of magnetic force caused by the magnet placed outside the furnace. The deviation between the furnace temperature and the temperature at which the sample is suppose to loose its magnetic property is the measure of error between the sample temperature and the one that is being recorded by the furnace. ICTA has come up with some recommended Ferro magnetic samples for the calibration of TG/DTA instruments [Wunderlich 1990]. We conducted this calibration procedure over the entire range of experimental temperature at various heating rates. The maximum deviation was within $\pm 3^{\circ} \mathrm{C}$. Once a calibration for temperature has been performed, then most of the thermobalances are capable of providing very precise data for long periods of time if the system is maintained properly, i.e., there is no contamination of the sample crucible, thermocouple, furnace wall and the balance mechanism has not been disturbed. However, it is important to perform these calibrations periodically. 


\subsection{Collection of Samples}

The samples reported in this Atlas were collected from a wide variety of sources specializing in various aspects of biomass. The sources are identified in appendix. In general the samples were reduced to +10 to -100 mesh by milling or grinding. Wherever it was necessary to obtain an average of a large specimen, whole plants were dried and milled. These samples are stored in viles and can be made available to interested parties for the sake of comparative testing.

\subsection{Errors in thermal analysis}

In the TG data collection many aspects have to be looked for before the data can be said to be the true representation of the decomposition kinetics or for that matter any other gas solid reaction. The common sources or error are

1. The presence of temperature gradients in the sample

2. Use of an excessive heating rate

3. The existence of a partial pressure of the product gases over the sample

4. The effect of impurities and mineral content

5. Buoyancy effects.

We will discuss in brief some of the effects due to these conditions in the TG/DTA data collection.

\subsubsection{The presence of temperature gradients in the sample}

If there is a temperature gradient within the sample from the surface to the center then the TG data collected for the decomposition cannot provide the correct decomposition kinetics because the sample temperature is not defined. This aspect limits the size of the sample and the heating rate to which it should be exposed. One way to correct for this error would be to go for lower heating rates with the increasing sample size. However, the isothermality of the sample can be defined in terms of a non-dimensional quantity called Biot number $\left(N_{B i}\right)$. It is the ratio of the external heat transfer to the surface of the sample to the internal heat transfer within the sample by thermal conduction

$$
\mathrm{N}_{\mathrm{Bi}}=\mathrm{h} / \mathrm{kr}
$$

where $h$ is the heat transfer coefficient to the sample surface, $\mathrm{k}$ is the thermal conductivity and $r$ is a characteristic dimension (the minimal diameter) of the sample. The higher the value of $\mathrm{N}_{\mathrm{Bi}}$ the greater is the temperature gradient within the sample. It has been found experimentally that temperature gradients become negligible $(<5 \%)$ at $\mathrm{N}_{\mathrm{Bi}}<0.1$ [Wilty et al. 1985]

\subsubsection{Use of an excessive heating rate}

Most biomass decomposition is either a simple decomposition of components such as cellulose, hemicellulose or lignin or the decomposition of mixtures of these. In the case of 
some biomass species the decomposition of individual components tend to overlap which makes it difficult to distinguish one from the other. This is also true for some polymeric materials like poly vinylchloride found in municipal solid waste. Polyvinyl Chloride, has two or three reactions in series. If the heating rate of the sample is very high say $100^{\circ} \mathrm{C} / \mathrm{min}$. then these reactions tend to overlap on the thermograms and one would then tend to interpret this as a single step decomposition. This leads to erroneous determination of kinetic parameters. It is therefore advisable to conduct experiments for decomposition kinetics under slow heating rates. The London Geological congress recommends the use of $10^{\circ}$ $\mathrm{C} / \mathrm{min}$. It has been found that this heating rate works good for most of the biomass samples. However, it is up to ones own judgment if as to what heating rate is best. For complex reactions such as mixture of two of three species it is advisable to test samples at heating rates as low as $1^{\circ} \mathrm{C} / \mathrm{min}$. ICTA recommends the reporting of heating rate along with every thermogram.

\subsubsection{The existence of a partial pressure of the product gases over the sample}

For reactions which are reversible in nature, such as the decomposition of $\mathrm{CaCO}_{3}$ to $\mathrm{CaO}$ and $\mathrm{CO}_{2}$, a partial pressure of the product gases would cause the slowing of the forward kinetics [Varhegyi, 1988]. It is therefore, important to keep the concentration of the product gases at a value where the kinetics of the reversible reaction has negligible effect on the decomposition process. This can be accomplished by keeping the flow rate of sweep gas sufficiently high. Care should be taken in doing this because at high flow rates for the sweep gas the errors due to buoyancy effect can become dominant and increase the contribution of systematic error on the data collection. For reactions which are not reversible in nature like the depolymerization of PVC one would think that low flow rates of sweep gas will not contribute to significant error. This thinking may lead to some problems because the vapor pressure of the product gases in some cases can have a catalytic effect on the decomposition process.

\subsubsection{The effect of impurities and mineral content}

Impurities such as ash mineral matter has an effect on the kinetics of decomposition. Therefore, to determine the true kinetics of the decomposition it is advisable to remove the ash content by any method such as acid wash followed by the neutralization with an alkali [Varhegyi and Antal, 1993]. However, it is true that the naturally occurring mineral matter would in most cases be present in actual application, so in this case one should determine the kinetics in the presence of the mineral matter. In such cases it is advisable to report the source and treatment of the sample. It is well known that samples of same species of biomass samples may have different inorganic contents, depending upon the geographic location from which they come from. This change is largely due to the geological form for forestry plants and the change in the fertilizer type for the agricultural crops. The analysis presented in this Atlas is without doing any pretreatment to the sample and therefore wherever possible the source of the sample has been listed. 


\subsubsection{Buoyancy effects}

Another important source of error in TG data collection relates to the buoyancy effects due to change in gas density at increasing temperatures. The mass change in the gas can be calculated by the application of ideal gas law and be used as a buoyancy correction factor.

\section{Note to readers}

Because of these various factors which can contribute to errors in TG/DTA data collection it has been found that the results from two laboratories can be somewhat different for the same sample operated under presumably identical conditions. In view of these differences in the literature, ICTA recommends a procedure for data reporting which we have reproduced in Chapter 9. 


\section{References}

[Abatzoglou, 1992] Abatzoglou, N., Chornet, E., Belkacemi, K and Overend, R. P Phenomenological Kinetics of Complex Systems: The Development of a Generalized Severity Parameter and its Application to Lignocellulosics Fractionation, Chemical Engineering Science, Vol 47, 1109, 1992.

[Agrawal, 1986] Agrawal, R. K., Journal of thermal analysis, Vol 31, p. 1253, 1986.

[Antal, 1982] Antal, M. J., Jr., Biomass Pyrolysis: A Review of the Liiterature Part 1Carbohydrate Analysis in Advances in Solar Energy ed. K. W. Boer and J. A. Duffie (American Solar Energy Society, Boulder, CO) Vo. 1, pp. 61-112, 1982.

[Antal, 1985] Antal, M. J., Jr., Biomass Pyrolysis: A Review of the Litterature Part 2Lignocellulose Pyrolysis in Advances in Solar Energy ed. K. W. Boer and J. A. Duffie (American Solar Energy Society, Boulder, CO) Vo. 1, pp. 175-255, 1985.

[Antal, 1994a] Antal, M. J., Jr., Cellulose Pyrolysis Kinetics: The Current State of Knowledge, Ind. Eng. Chem. Res. in press, 1994.

[ASTM, 1989] See: 2013-86, Standard Method of Preparing Coal Samples for Analysis; 3172-73, Standard Method for Proximate Analysis of Coal and Coke; 3173-87; Standard Test Method for Moisture in the Analysis of Samples of Coal and Coke; 3175-89, Standard Test Method for Volatile Matter in the Analysis of Samples of Coal and Coke; 3174-89, Standard Test Method for Ash in the Analysis of Samples of Coal and Coke; 3176-84, Standard Method for Ultimate Analysis of Coal and Coke; 3177-84, Standard Test Method for Total Sulfur in the Analysis of Samples of Coal and Coke; 3178-84, Standard Test Methods for Carbon and Hydrogen in the Analysis of Samples of Coals and Cokes; 3179-84, Standard Test Methods for Nitrogen in the Analysis of Samples of Coal and Coke; 2015-85, Standard Test Method for Gross Calorific Value of Coal and Coke by the Adiabatic Bomb Calorimeter, in Coal and Coke, Section 5, Vol.05.05, Annual Book of ASTM Standards (1989).

[Bain, 1981] Bain, R., Beneficiation of Biomass for Gasification and Combustion, in Biomass gasification: Principles and Technology, Ed. T.B. Reed, Noyes data corp. Park ridge, NJ, USA, 1981.

[Bateman 1953] Bateman, H., and Erdelyi, A., Higher transcendental functions, McGraw Hill Co., Vol. 2, 1953.

[Belkacemi, 1991] Belkacemi, K., Abatzoglou, N., Overend, R. P. and Chornet, E., Phenomenological Kinetics of Complex Systems: Mechanistic Considerations in the Solubilization of Hemicelluloses following Aqueous/Steam Treatments, I\&EC Research Vol 30, 2416, 1991.

[Berger, 1951] Berger I. A. and Whitehead W. L., Fuel, Vol. 30, p.247, 1951.

[Blumberg, 1959] Blumberg, A. J., J. Phys Chem., Vol. 63, p. 1129, 1959. 
[Borchardt, 1956] Borchardt, H. J., Ph.D. Thesis, University of Wisconsin, Madison, Wisconsin, 1956.

[Borchardt, 1957] Borchardt, H. J. and Daniels F. J., Am. Chem. Soc., Vol. 79, p. 41, 1957. [Bridgwater, 1994] Bridgwater, A. V., Ed., Advances in Thermochemical Biomass Conversion Blackie Academic And Profesional, London, 1994.

[Broido, 1969] Broido, A., Journal of Polymer Science, Part A2, Vol. 7, p. 1761, 1969. [Cardwell, 1976] Cardwell, R. D. and Luner, P., Wood Science and Technology, Vol. 10, p. 183, 1976.

[Channiwala, 1992] Channiwala, S. A., On the Biomass Gasification Process, Ph.D. Thesis, Indian Institute of Technology, Department of Mechanical Engineering, Bombay, India, 1992.

[Chatterjee, 1965] Chatterjee, P. K., Journal of Polymer Science, Vol. A3, p. 4253, 1965.

[Chiu, 1962] Chiu, J., Anal. chem., Vol. 34, no. 13, p. 1841, 1962.

[Chiu, 1966] Chiu, J., Thermoanalysis of fiber and fiber forming polymers, Ed. R.F. Schwenker, Interscience, NY, p. 25, 1966.

[Chornet, 1980] Chornet, E., Roy, C., " Compensation Effect in the Decomposition of Cellulosic Materials", Thermochemica Acta Vol 35, 389-393, 1980.

[Coats, 1964] Coats, A. W. and Redfern, J. P., Nature, Vol. 201, p. 68, 1964.

[Diebold, 1984] Diebold, J. P., Ablative Entrained Flow Fast Pyrolysis of Biomass, in 16th Biomass Thermochemical Conversion Contractors Meeting, Portland Or, May 1984.

[Diebold, 1985] Diebold, J. P., The Cracking Kinetics of Depolymerized Biomass Vapors in a Continuous Tubular Reactor, Thesis T 3007, Colorado School of Mines, Golden, CO 1985.

[Diebold, 1988] Diebold, J. P. and Schahill, J. W., Energy progress, Vol. 8, no. 1, p. 59, 1988.

[Doyle, 1961] Journal of Applied Polymer Science, Vol. V, No. 15, p. 285, 1961.

[Duval, 1951] Duval, C., Anal. chem, Vol. 23, p. 1271., 1951.

[Earl, 1974] Earl, D. E., A report on charcoal, FAO of UN, ISBN 92-5-10024-X, 1974.

[Freeman, 1958] Freeman D. and Carroll, J. Chem. Phys. Vol 62, 394 (1958)

[Friedman, 1965] Friedman, H. L., Analytical chemistry, Vol. 37, p. 768, 1965.

[Fritsky, 1994] Fritsky, K. J., Miller, D. L. and Cernansky, N. P., J. Air and waste Mgmnt. Assn. Vol 44, 11161994.

[Flynn, 1966] Flynn, J. H. and Wall, L. A., Journal of research of NBS, Vol. 70A, No. 6, p. 487, 1966.

[Gadalla, 1985] Gadalla, A. M., Thermochimica Act, Vol. 95, p. 179, 1985.

[Garn, 1957] Garn, P.D. and Flashen, S. S., Anal. chem, Vol. 29, p. 271, 1957.

[Gaur, 1993] Gaur S. and Reed, T. B., ACS National Symposium, Denver, Cellulose and Textile Div., abstract no. 25, (Also in Biomass and Bioenergy, in press), 1993. 
[Glass, 1955] Glass, H. D., Fuel, Vol. 34, p. 253, 1955.

[Graboski, 1981] Graboski, M. S., Properties of Biomass Relevant to Gasification, in Biomass Gasification: Principles and Technology, Ed. T. B. Reed, Noyes Data Corp., Park Ridge, NJ., USA, in Biomass gasification: Principles and Technology, Ed. T.B. Reed, Noyes data corp. Park ridge, NJ, USA, p 41, 1981.

[Graham, 1988] Graham, R. G., Freel, B. A., Huffman, D. R. and Bergougnou, R. Applications of Thermal Processing of Biomass, in [Bridgwater, 1994]

[Gyulai, 1074] Gyulai, G. and Greenhow, E. J., Journal of Thermal Analysis, Vol. 6, p. 279, 1974.

[Hemingway, 1988] Adhesives from Renewable Resources, Eds. Hemingway and Connor; ACS Monograph 385. ??

[Hoffman, 1959] Hoffman, I., Schnitzer, M. and Wright, J. R., Anal. Chem, Vol. 31, p. 440., 1959.

[Hollings, 1923] Hollings, H. and Cobb J. W., Fuel, Vol. 2, p. 322, 1923.

[IGT, 1978] Institute of Gas Technology, Coal Conversion Systems Technical Data Book, DOE Contract EX-76-C-01-2286, 1978.

[IHI, 1993] The International Humic Institute is operated by Prof. Patrick McCarthy and is located at the Colorado School of Mines in Golden, CO., (80401).

[Inman, 1981] Inman, R., The Potential Biomass Resource Base, in Biomass gasification: Principles and Technology, Ed. T.B. Reed, Noyes data corp. Park ridge, NJ, USA, 1981.

[IPST, 1993] Sampies obtained from Mr. Alan Rudie (404-853 9706) at the Institute for Paper Science and Technology in Atlanta, Ga.

[Jenkins, 1985] Jenkins, B. M. and Ebeling, J. M., Correlation of Physical and Chemical Properties of Terrestrial Biomass with Conversion, Symposium, Energy from Biomass and Waste IX, IGT, p. 371, 1985.

[Kirk, 1983] Kirk and Othmer, Carbohydrates, Encyclopedia of Chemical Technology, Vol. 1, 1983.

[Kissinger, 1957] Kissinger, H. E., Anal. Chem, Vol. 29, p. 1702. 1957.

[LeChaterlier, 1887] LeChatelier, H., Bull. Soc. Fr. Mineral Cristallogr. 1887, Vol. 10, p. 204211, translated by Dr. L. E. Nesbitt, Southern Colorado state college, Pueblo, Colorado. ?? [Liptay, 1972] Liptay, G., Atlas of Thermoanalytical Curves, Heyden and Sons Ltd., NY, 1972.

[Mackenzie, 1970] Mackenzie, R. C., Differential thermal analysis, Vol. I Academic press, 1970.

[Milne, 1981] Milne, T. Pyroysis - the Thermal Behavior of Biomass Below $600^{\circ} \mathrm{C}$, in Biomass Gasification: Principles and Technology, Ed. T. B. Reed, Noyes Data Corp., Park Ridge, NJ., USA, in Biomass gasification: Principles and Technology, Ed. T.B. Reed, Noyes data corp. Park ridge, NJ, USA, p 91, 1981. 
[Murphy, 1058] Murphy, C. D., Anal. Chem, Vol. 30, p. 967, 1958.

[Norton, 1939] Norton, F. H., J. Am. Ceramic Soc., Vol. 22, p. 54, 1939.

[NREL, 1994] Biomass Pyrolysis Oil, Properties and Combustion, Conference, proceedings to issue 1994-95, NREL/CP-433-7265.

[OTA, 1980] Energy from Biological Processes, OTA-E-124, Office of Technology Assessment, Washington, D.C. 1980.

[Ramachandran, 1954] Ramachandran, V. S. and Bhattacharya, S. K., J. of Sci and Ind. research, India, Vol. 13A, p. 365, 1954.

[Reed, 1981] Reed, T. B., Biomass Gasification : Principles and Technology, Noyes Data Corp., Park Ridge, NJ., USA, 1981. (Also in "A Survey of Biomass Gasification", 3 volumes, SSERI/TR-33-239, 1979.

[Reed, 1985] Reed, T. B., Biomass Gasification in Advances in Solar Energy ed. K. W. Boer and J. A. Duffie (American Solar Energy Society, Boulder, CO) Vo. 1, p. 158, 1985.

[Reed, 1994] Reed, T. B., Mobeck, W. L. and Gaur, S., A Hydrophobic Oleophilic Form of Biomass for Oil and Chemical Absorption, in Advances in Thermochemical Conversion, Ed. A.V. Bridgwater, Blackie Academic and Professional, Vol. 2, p. 1214, 1994.

[Reed, 1993] Reed, T. B., An Overview of the Current Status of Biodiesel, in Proceedings of The First Biomass Conference of the Americas, Aug. 30, 1993. ??

[Reed, 1992] Reed, T. B., Graboski, M. S. and Gaur, S., Biomass and Bioenergy, Pergamon press, UK, Vol. 3, no. 2, p. 111, 1992.

[Richard, 1994] Richard J.R. and Antal, M. J., Advances in Thermochemical Conversion, Ed. A.V. Bridgwater, Blackie Academic and Professional, Vol. 2, p. 784, 1994.

[Risser, 1981] Risser, P. G. Agricultural and Forestry Residues in BiomassConversion Processes for Energy and Fuels, Ed. Soffer, S. S. and Zaborsky, O. R., Plenum Press, N.Y., pp 25-56, 1981.

[Roberts, 1899] Roberts-Austen, W. C., Proceeding of Institute of Mech. Engineers, Vol. 1, p. $35,1899$.

[Rossi, 1984] Rossi, A., Fuel Characteristics of Wood and Non-Wood Biomass Fuels, in Progress in Biomass Conversion, Ed. Tillman, D. A. and Jahn, E. C., Vol 5, Academic Press, Inc., p 69, 1984.

[Sanderman, 1063] Sanderman, W. and Augustine, H., Holzroh U-werkstoff, Vol. 21 p. 305 , 1963.

[Satava, 1969] Satava, V. and Skvara, F., Journal of American Chemical Society, Vol. 52, No. 11, p. $591,1969$.

[Schwenker, 1960] Schwenker, R. F., and Beck, L. R., Textile Research Journal, Vol. 30, p. 624, 1960.

[Shafizadeh, 1979] Shafizadeh, F., Furneaus, R. H. and Cochran, T. G. Scholl, J. P., Sakai, Y., J. of Appl. Polym. Sci. Vol 23, 3525-3539. 
[Shafizadeh, 1982] Shafizadeh, F. J. Anal. Appl. Pyrol., Vol3, 283, 1982.

[Soltes, 1988] Pyrolysis Oils from Biomass, Eds. E. J. Soltes and T: A. Milne, AACS Symposium Series 376, Washington D. C. 1988.

[Stevens, 1944] Stevens, D. J. Review and Alanlysis of the 1980-1989 Biomass

Thermochemical Conversion Program, NREL Subcontract AAE-3-13029-01, 1994.

[Swern, 1979] Swern, D., Bailey's Industrial Oil and Fat Products, 2 Volumes, 4th Ed., J. Wiley and Sons, NY., 1979.

[Tateno, 1966] Tateno, J., Trans. Faraday Soc., Vol. 62, p. 1885, 1966.

[Tillman, 1978] Tillman, D. A., Wood As an Energy Resource, Academic Press, NY, 1978.

[Varhegyi, 1978] Varhegyi, G., Thermochimica Acta, Vol. 25, p. 201, 1978.

[Varhegyi, 1988] Varhegyi, G., Szekely, T., Till, F., Jakab, E. and Szabo, P.; J. of Thermal Analysis, Vol. 33, p. 87, 1988.

[Varhegyi, 1993]Varhegyi, G, Szabo, P. and Antal, M.J., ACS National Symposium, Denver, Cellulose and Textile Div., abstract no. 12, (Also in Biomass and Bioenergy, in press), 1993.

[Varhegyi, 1994] Varhegyi, G., Jakab, E. and Antal, M. J. Jr., Is the Broido-Shafizadeh Model for Cellulose Pyrolysis True?, Energy and Fuels, to be published, 1994.

[Weber, 1982] Weber, D. R., "Energy Information Guide, Vol 1: General and Alternative Information Sources", ABC-Clio, Santa Barbara, Calif., 1982.

[Wendlendt, 1961] Wendlendt, W. W., J of Chemical Education, Vol. 38, p. 571, 1961.

[Wilty 1984] Wilty, J. R., Wicks, C. E. and Wilson, R. E., Fundamentals of momentum, heat and mass transfer, John Wiley and Sons, 3rd ed, p. 299., 1984.

[Wittels, 1951] Wittels, M., Amer. Minerologist, Vol. 36, p. 615, 1951.

[Wunderlich, 1974] Wunderlich, B. and Bopp, R.C., J Thermal Analysis, Vol. 6, p. 335, 1974.

[Wunderlich, 1990] Wunderlich, B., Thermal analysis, Academic Press, p. 371, 1990.

[Zsako, 1970] Zsako, J., Journal of Thermal Analysis, Vol. 2, p. 145, 1970.

[Zsako, 1973] Zsako, J., Journal of Thermal Analysis, Vol. 5, p. 239, 1973. 


\section{INDEX}

About the Atlas

Agricultural Residues

Applications of differential thermal analysis

Applications of thermogravimetric analysis .

Aquatic Biomass

Babool wood (Acacia arabica, India)

Bass wood (Tilia american)

Biodiesel

Biodiesel (Corn oil - methyl ester)

Biomass components additivity (populus deltoides) .............................................. 8-6

Black locust (Robina pseudoacacia)................................................................... 2-18

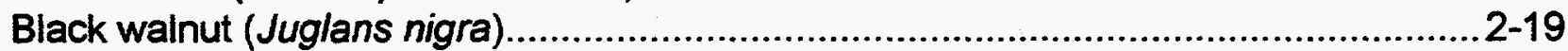

Brown rotted wood (S. pinus by P. placenta) ...................................................... 7-18

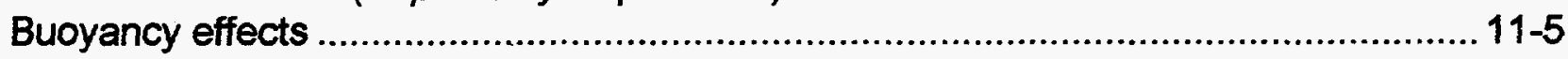

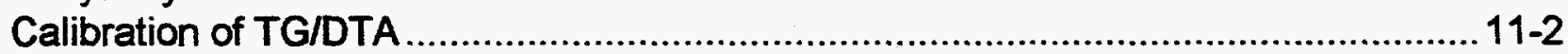

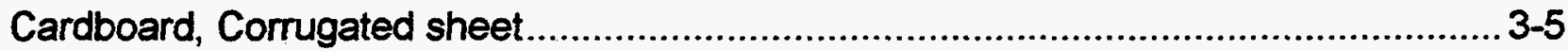

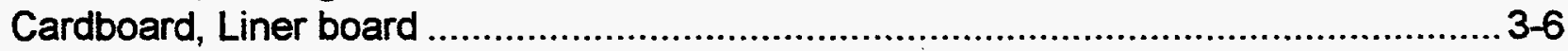

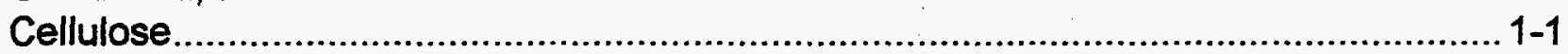

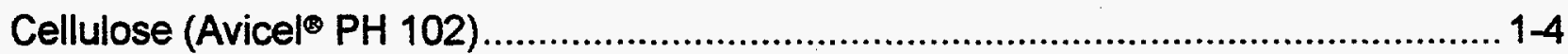

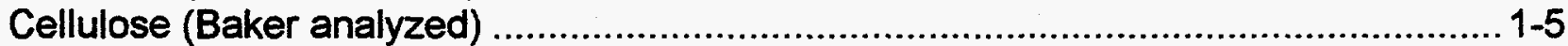

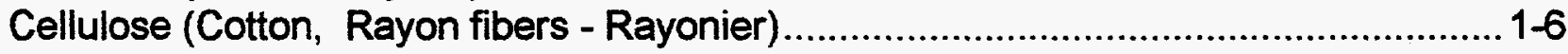

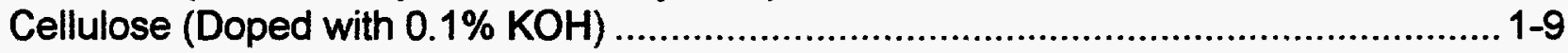

Cellulose (Doped with $0.1 \% \mathrm{ZnCl}_{2}$ ) ............................................................. 1-10

Cellulose from Bacteria (Acetobactor xylinum cellulose) ...........................................1-8

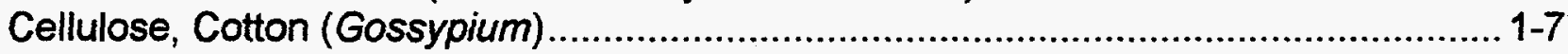

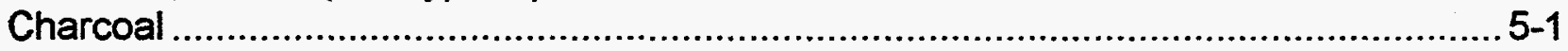

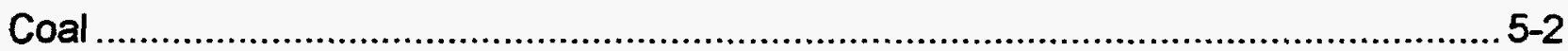

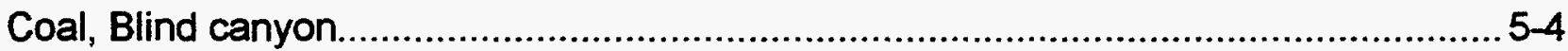

Coal, Buelah zap lignite (North Dakota) ............................................................... 5-5

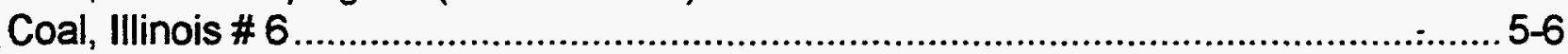

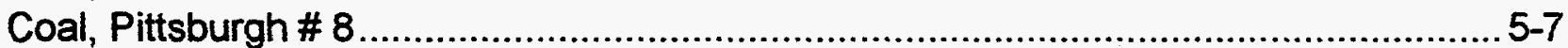

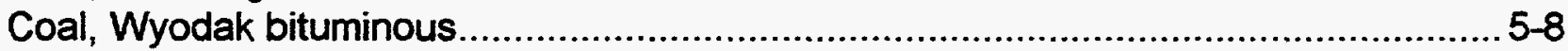

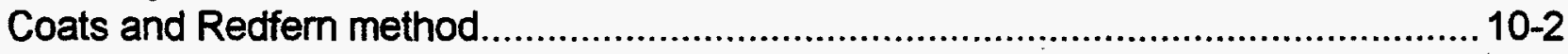

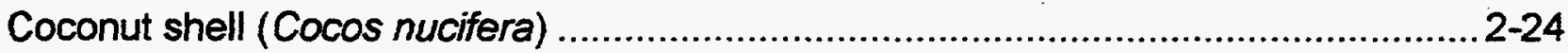

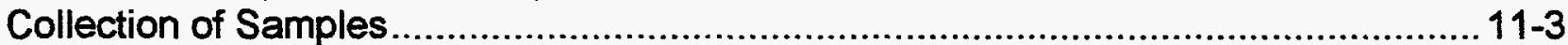

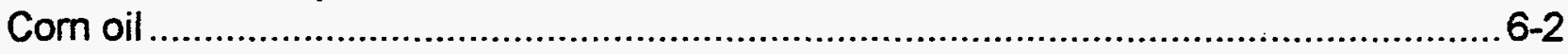

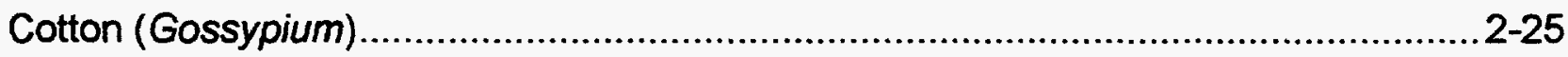

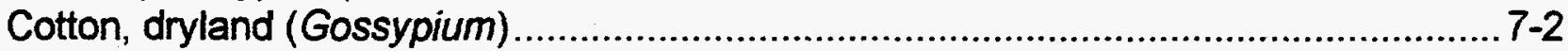

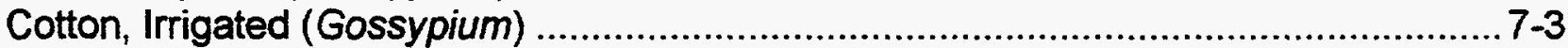

Densified biomass ...........................................................................................

Determination of kinetic data using thermogravimetric data .............................. 10-2

Determination of kinetic parameters using differential thermal analysis

Diesel fuel. 
Differential thermal analysis and Differential scanning calorimetry ............................9-4

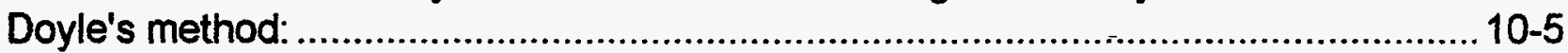

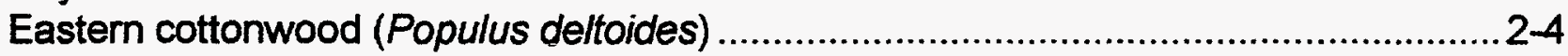

Eastern cottonwood stored for 26 weeks (Populus deltoides) ................................... $7-6$

Eastern cottonwood stored for zero weeks (Populus deltoides) ................................ 7-5

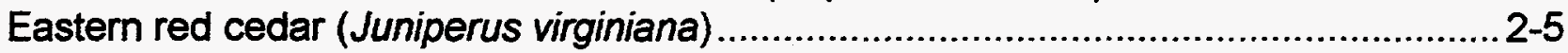

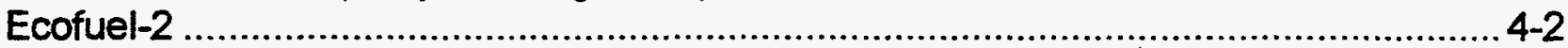

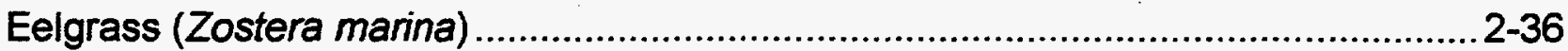

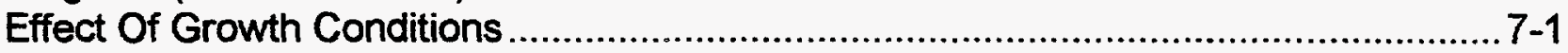

Effect of growth conditions (Dryland \& irrigated cotton) ......................................... $7-4$

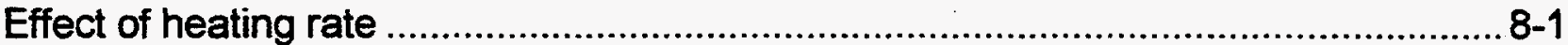

Effect of heating rate (Pelletized Baker Cellulose) .................................................. 8-4

Effect of heating rate (Western red cedar) .......................................................... 8-3

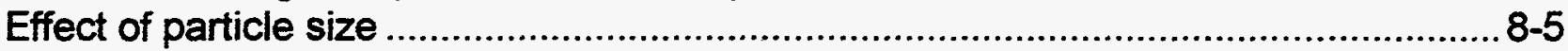

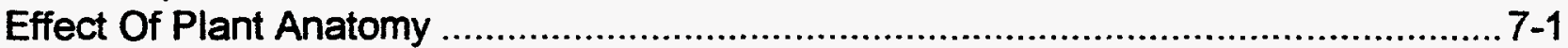

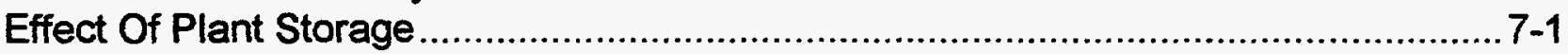

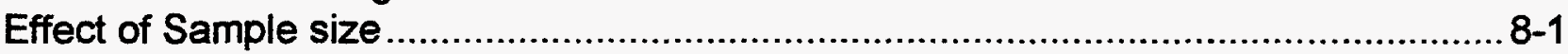

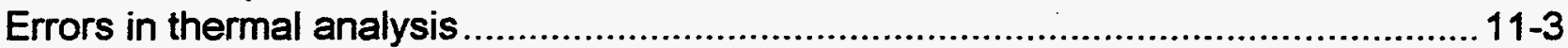

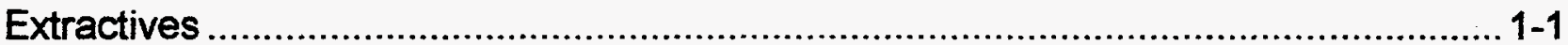

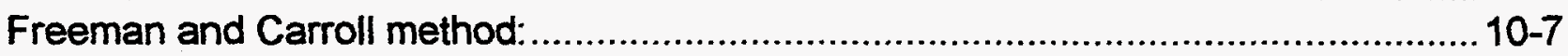

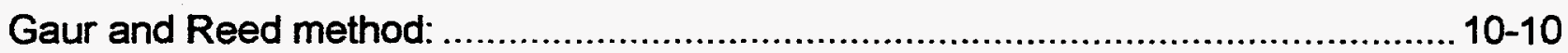

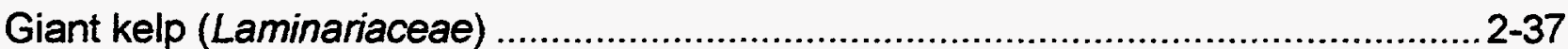

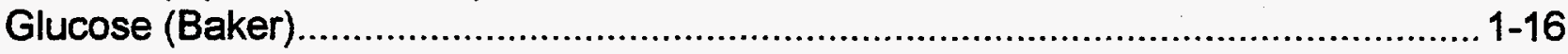

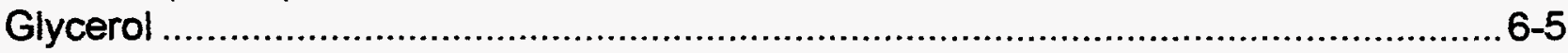

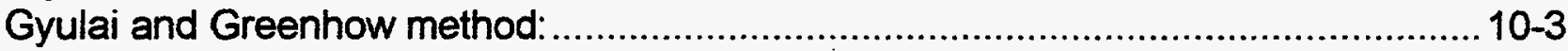

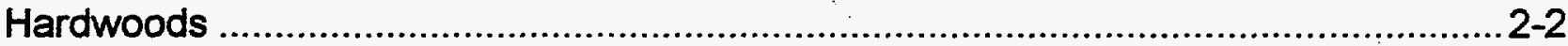

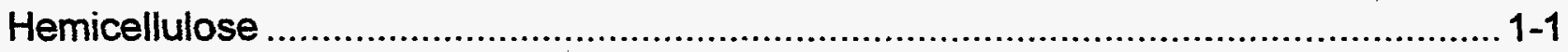

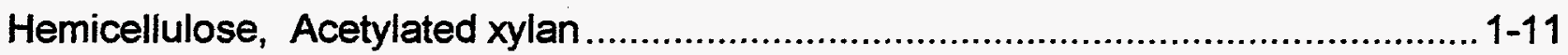

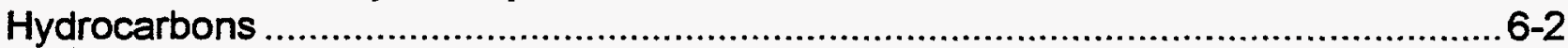

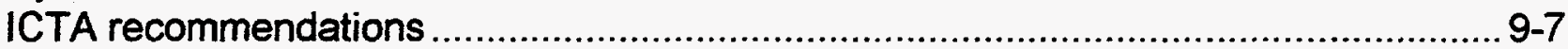

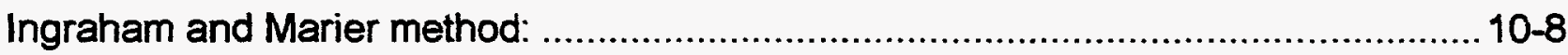

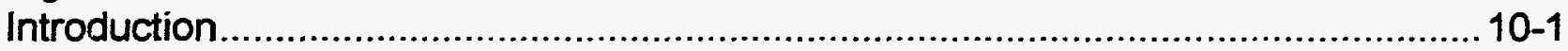

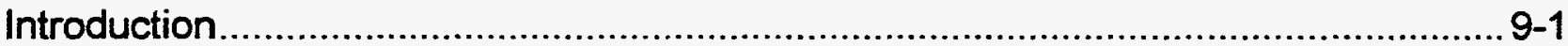

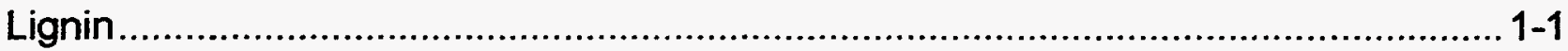

Lignin, Ball milled wood (populus deltoides) .................................................. 1-13

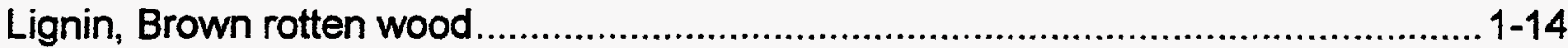

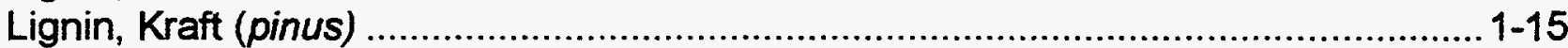

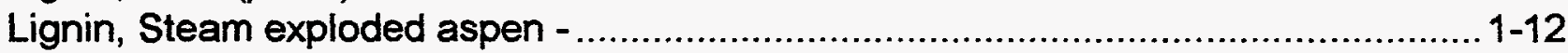

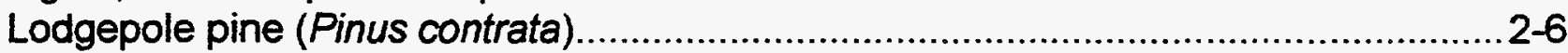

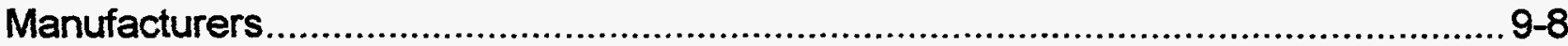

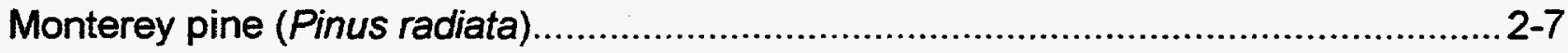

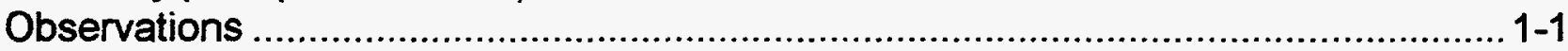

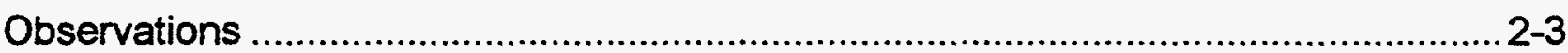

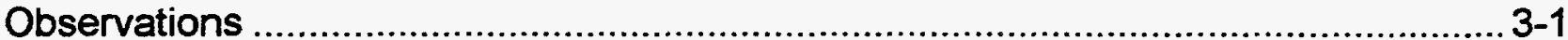

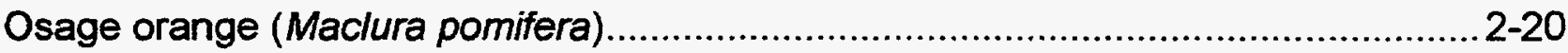

Other effects in understanding DTA data .......................................................10-13 


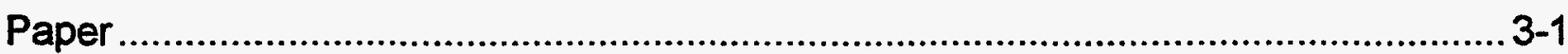

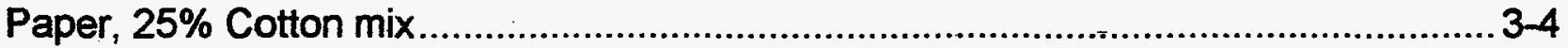

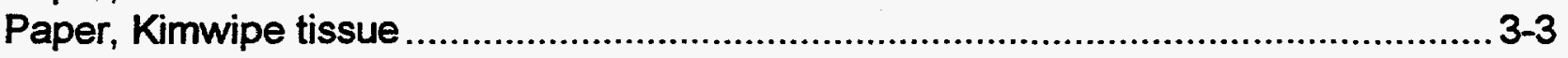

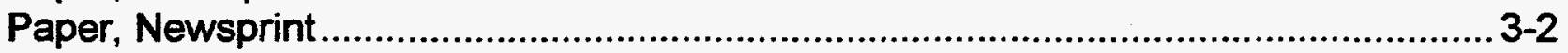

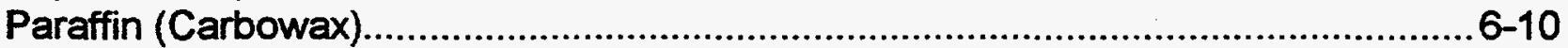

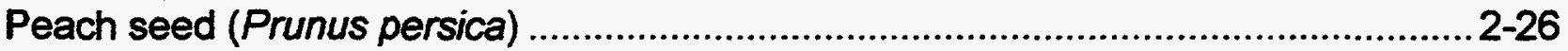

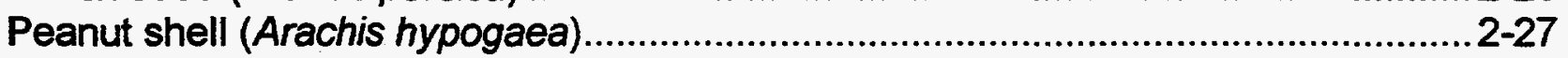

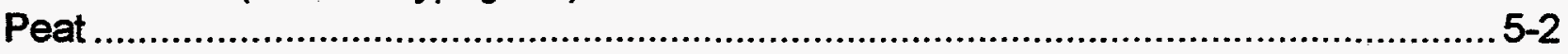

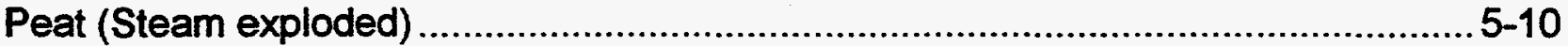

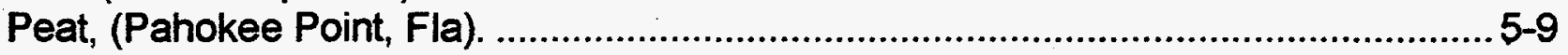

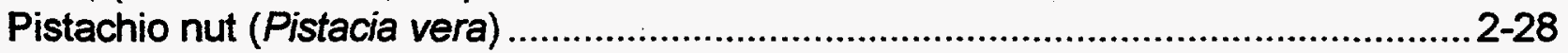

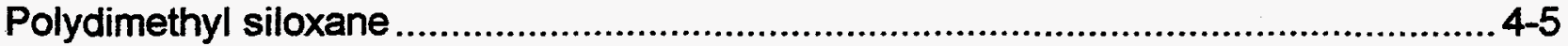

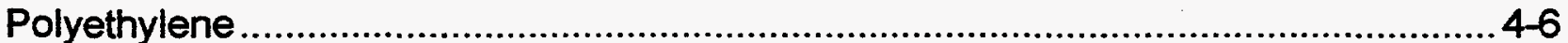

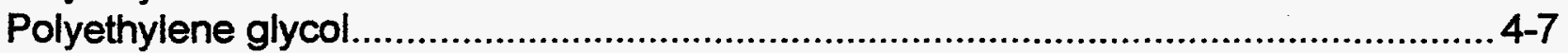

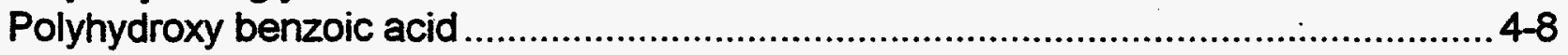

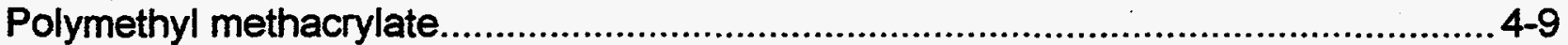

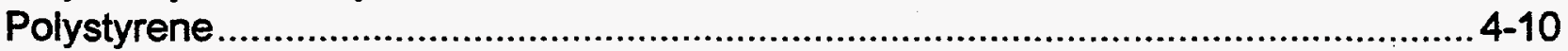

Polytetrafluroethylene (Teflon) ....................................................................... 41

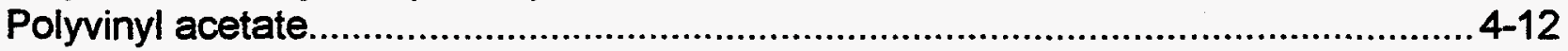

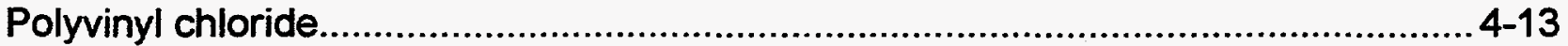

Ponderosa pine (Pinus ponderosa) ................................................................... 2-8

Ponderosa pine bark (Pinus ponderosa) ............................................................ 2-9

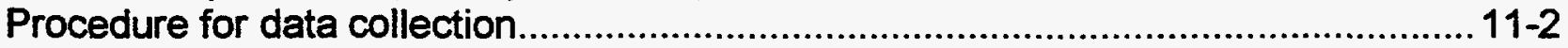

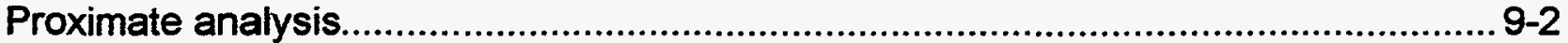

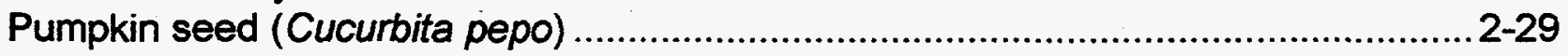

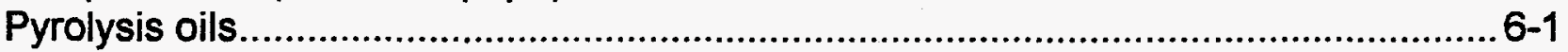

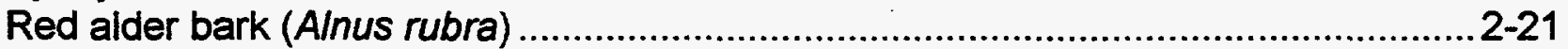

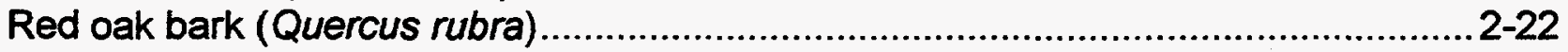

Red Oak Pyrolysis oil (Quercus rubra) .......................................................................6-6

Redwood (Sequoia sempervirens) ................................................................... 2-10

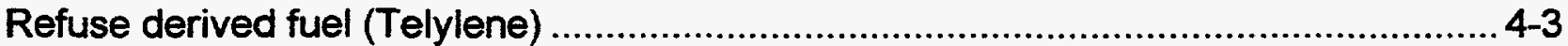

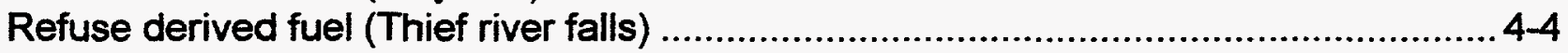

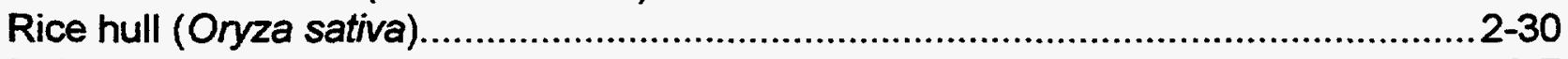

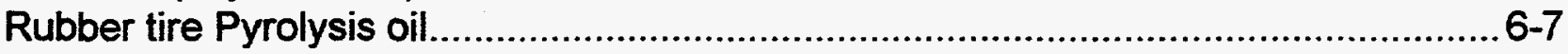

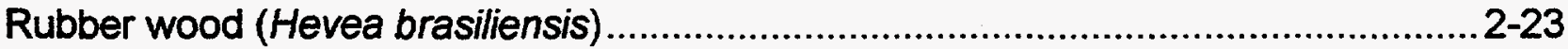

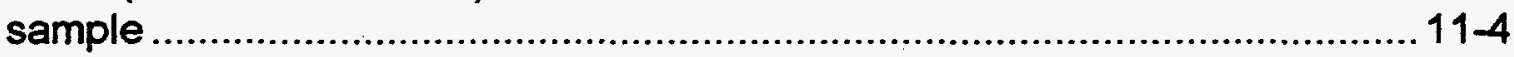

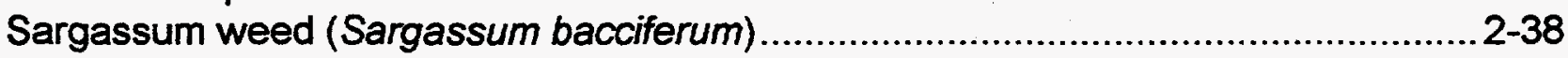

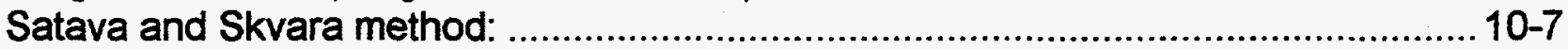

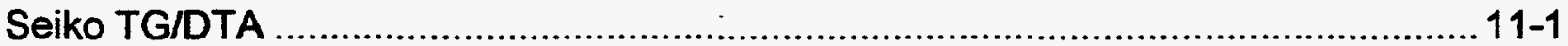

Sericea lespedeza (Lespedeza cuneata var. Serala) ...........................................2-31

Sericea lespedeza stored for 26 weeks (Lespedeza cuneata) ...................................7-8

Sericea lespedeza stored for zero weeks (Lespedeza cuneata) ................................ 7-7

Slash pine (Pinus elliotti) .................................................................................. 2-11

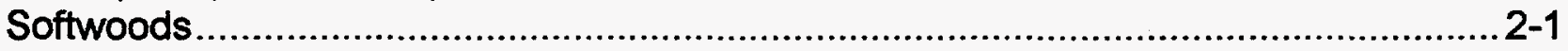

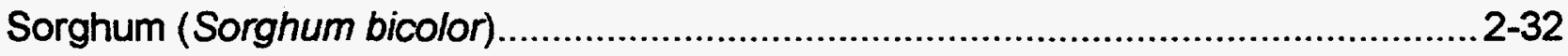

Sorghum stored for 26 weeks (Sorghum bicolor ................................................ $7-10$ 
Sorghum stored for zero weeks (Sorghum bicolor) ................................................. 7-9

Spinace Stem (Spinacea oleracea) .................................................................... 2-33

Spinach cell cytoplasmic liquid (Spinacea oleracea) ............................................. 7-13

Spinach stem cell chloroplast (Spinacea oleracea) .............................................. 7-12

Spinach stem xylem and phloem (Spinacea oleracea) …......................................... 7-11

Sugar cane baggase (Saccharum officinarum) ................................................... 2-34

Sugar cane baggase stored for 26 weeks (Saccharum officinarum) .......................... 7-15

Sugar cane baggase stored for zero weeks (Saccharum officinarum) ....................... 7-14

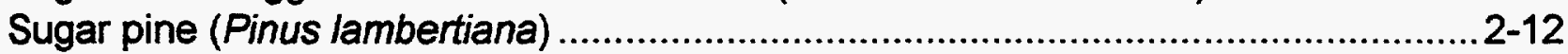

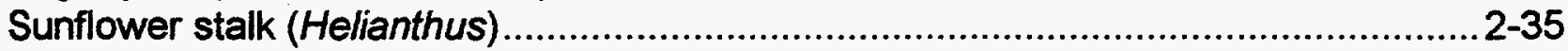

Switchgrass (Panicum virgatum L.) …................................................................... 2-36

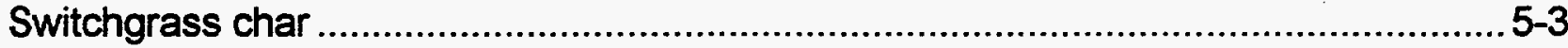

Switchgrass Pyrolysis oil (Panicum virgatum L.) ...................................................6-8

Switchgrass stored for 26 weeks (Panicum virgatum L.) ....................................... 7-17

Switchgrass stored for zero weeks (Panicum virgatum L.) .................................... 7-16

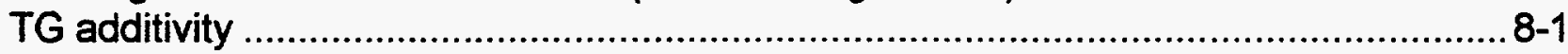

The effect of impurities and mineral content.......................................................11-4

The existence of a partial pressure of the product gases over the

The presence of temperature gradients in the sample ............................................11-3

Thermogravimetry and Differential thermogravimetry ............................................. 9-2

Trembling aspen (Populus tremuloides) ............................................................. 2-13

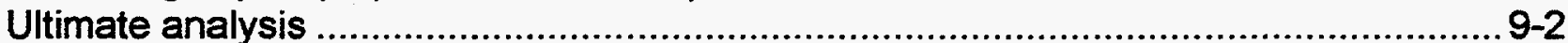

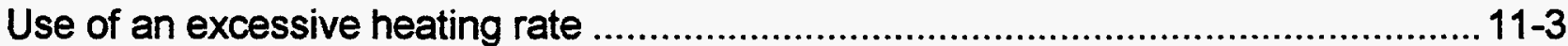

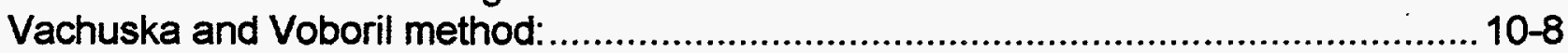

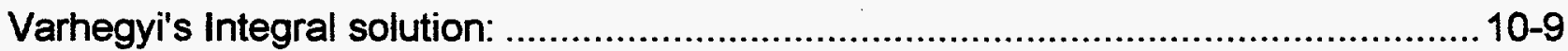

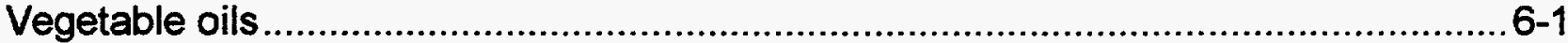

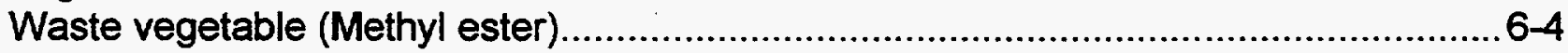

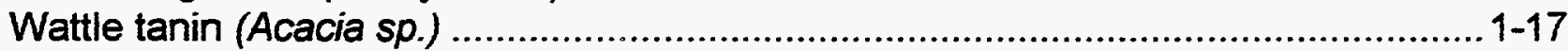

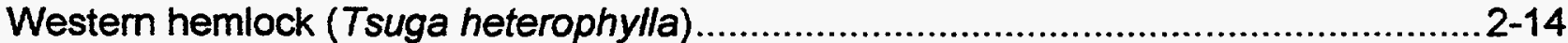

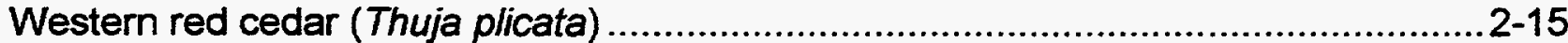

Wheat straw (Triticum aestivum, var Thunderbird) ................................................2-37

White rotted wood (White spruce by Ganoderma applanatum .............................. 7-19

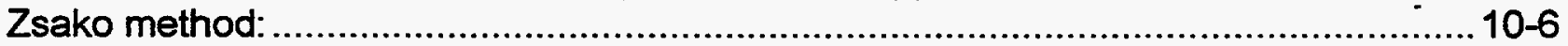




\section{APPENDIX A - PROXIMATE, ULTIMATE ANALYSIS AND HIGH HEATING VALUE OF SELECTED BIOMASS AND OTHER FUELS ${ }^{5}$}

NAME

Ash, White
Beech
Birch, White
Black Locust
Canyon Live Oak
Cedar, White
Chaparall
Chinkapin
Cypress
Douglas Fir
Douglas Fir
Elm
Hickory
Madrone
Madrone
Mango Wood
Manzanita
Maple
Pine, White
Plywood
Ponderosa Pine

ULTIMATE ANALYSIS

PROXIMATE

ANALYSIS

\begin{tabular}{|c|c|c|c|c|c|}
\hline $\begin{array}{c}\text { Fixed } \\
\text { Carbon } \\
\%\end{array}$ & $\begin{array}{l}\text { Vola- } \\
\text { tiles } \\
\%\end{array}$ & Ash & C & $\mathbf{H}$ & $\mathbf{0}$ \\
\hline & & \multicolumn{4}{|c|}{ WOOD } \\
\hline & & 0.30 & 49.70 & 6.90 & 43.00 \\
\hline & & 0.65 & 51.64 & 6.26 & 41.45 \\
\hline & & 0.30 & 49.80 & 6.50 & 43.40 \\
\hline 18.26 & 80.94 & 0.80 & 50.73 & 5.71 & 41.93 \\
\hline \multirow[t]{2}{*}{11.30} & 88.20 & 0.50 & 47.84 & 5.80 & 45.76 \\
\hline & & 0.40 & 48.80 & 6.40 & 44.40 \\
\hline 18.68 & 75.19 & 6.13 & 46.90 & 5.08 & 40.17 \\
\hline \multirow[t]{2}{*}{12.80} & 86.90 & 0.30 & 49.68 & 5.93 & 44.03 \\
\hline & & 0.40 & 55.00 & 6.50 & 38.10 \\
\hline 17.70 & 81.50 & 0.80 & 52.30 & 6.30 & 40.50 \\
\hline \multirow[t]{3}{*}{12.60} & 87.30 & 0.10 & 50.64 & 6.18 & 43.00 \\
\hline & & 0.70 & 50.40 & 6.60 & 42.30 \\
\hline & & 0.73 & 47.67 & 6.49 & 43.11 \\
\hline 15.10 & 84.50 & 0.30 & 48.56 & 6.05 & 45.08 \\
\hline 12.00 & 87.80 & 0.20 & 48.94 & 6.03 & 44.75 \\
\hline 11.36 & 85.64 & 2.98 & 46.24 & 6.08 & 44.42 \\
\hline \multirow[t]{3}{*}{17.89} & 81.29 & 0.82 & 48.18 & 5.94 & 44.68 \\
\hline & & 1.35 & 50.64 & 6.02 & 41.74 \\
\hline & & 0.10 & 52.60 & 6.10 & 41.20 \\
\hline 15.77 & 82.14 & 2.09 & 48.13 & 5.87 & 42.46 \\
\hline 17.17 & 82.54 & 0.29 & 49.25 & 5.99 & 44.36 \\
\hline
\end{tabular}

HIGH HEAT- ERROR REF

ING VALUE

$\mathbf{N}$

$\begin{array}{llll}\% & \% & \mathrm{~kJ} / \mathrm{mol} \quad \mathrm{kJ} / \mathrm{mol}\end{array}$

(M-C)/

$\mathbf{M}$

$\begin{array}{llllrc} & & 20.75 & 21.03 & -1.34 \% & 20 \\ 0.00 & 0.00 & 20.38 & 21.10 & -3.57 \% & 24 \\ & & 20.12 & 20.55 & -2.14 \% & 20 \\ 0.57 & 0.01 & 19.71 & 20.08 & -1.87 \% & 6 \\ 0.07 & 0.01 & 18.98 & 18.79 & 0.99 \% & 11 \\ & & 19.54 & 19.98 & -2.25 \% & 20 \\ 0.54 & 0.03 & 18.61 & 18.07 & 2.90 \% & 6 \\ 0.07 & 0.01 & 19.35 & 19.77 & -2.17 \% & 11 \\ & & 22.96 & 22.91 & 0.21 \% & 20 \\ 0.10 & 0.00 & 21.05 & 21.48 & -2.01 \% & 24 \\ 0.06 & 0.02 & 20.38 & 20.51 & -0.67 \% & 11 \\ & & 20.49 & 20.98 & -2.39 \% & 20 \\ 0.00 & 0.00 & 20.17 & 20.51 & -1.72 \% & 24 \\ 0.05 & 0.02 & 19.14 & 19.42 & -1.42 \% & 11 \\ 0.05 & 0.02 & 19.51 & 19.56 & -0.25 \% & 11 \\ 0.28 & & 19.17 & 18.65 & 2.73 \% & 20 \\ 0.17 & 0.02 & 19.30 & 19.18 & 0.62 \% & 6 \\ 0.25 & 0.00 & 19.96 & 20.42 & -2.33 \% & 24 \\ & & 20.70 & 21.29 & -2.83 \% & 20 \\ 1.45 & 0.00 & 18.96 & 19.26 & -1.62 \% & 5 \\ 0.06 & 0.03 & 20.02 & 19.66 & 1.80 \% & 6\end{array}$


NAME

Poplar

Red Alder

Redwood

Tan Oak

Tan Oak

Western Hemlock

White Fir

White Oak

Yellow Pine

Alfalfa seed straw

Almond Hulls

Almond Prunings

Almond Shells

Bagasse

Bagasse

Bean Straw

Black Walnut Prunings

Cabernet Sauvignon Pr

Chenin Blanc $\mathrm{Pr}$

Cocoa Hulls

Coconut fibre (Coir)

Coconut shells

Corn Stalks
PROXIMATE

Fixed Vola- Ash

Carbon tiles

$\% \quad \%$

$\begin{array}{rr}12.50 & 87.10 \\ 16.10 & 83.50 \\ 12.40 & 87.10 \\ 9.20 & 90.60 \\ 15.20 & 84.80 \\ 16.58 & 83.17 \\ 17.20 & 81.28\end{array}$

$0.65 \quad 5164$

$0.40 \quad 49.55$

$0.40 \quad 53.50$

$0.50 \quad 48.34$

$0.20 \quad 48.67$

$2.20 \quad 50.40$

$0.25 \quad 49.00$

$\begin{array}{ll}1.52 & 49.48\end{array}$

1.3152 .60

\section{AGRICULTURAL}

$\begin{array}{ll}20.15 & 72.60 \\ 22.89 & 71.33 \\ 21.54 & 76.83 \\ 21.74 & 73.45 \\ 16.87 & 75.10 \\ 22.10 & 64.60 \\ 18.77 & 75.30 \\ 18.56 & 80.69 \\ 19.20 & 78.63 \\ 20.21 & 77.28 \\ 23.80 & 67.95 \\ 29.70 & 66.58 \\ 22.10 & 77.19 \\ 20.30 & 73.30\end{array}$

\section{$\begin{array}{lll}7.25 & 46.76 & 5.40\end{array}$}

$5.78 \quad 45.79$

$\begin{array}{llll}1.63 & 51.30 & 5.29 & 40.90\end{array}$

$\begin{array}{lllll}4.81 & 44.98 & 5.97 & 42.27\end{array}$

$\begin{array}{llll}8.03 & 45.71 & 5.89 & 40.37\end{array}$

$\begin{array}{lllll}13.30 & 39.70 & 5.50 & 42.30\end{array}$

$\begin{array}{llll}5.93 & 42.97 & 5.59 & 44.93\end{array}$

$0.78 \quad 49.80$

$5.82 \quad 43.25$

$\begin{array}{ll}2.17 & 46.59\end{array}$

$\begin{array}{ll}2.51 & 48.02\end{array}$

$5.85 \quad 43.90$

$5.89 \quad 41.93$

$8.25 \quad 48.23$

$\begin{array}{ll}3.72 & 50.29\end{array}$

$0.71 \quad 50.22$

$6.40 \quad 43.86$

$\begin{array}{ll}5.23 & 33.19\end{array}$

$5.05 \quad 39.63$

$5.70 \quad 43.37$

$5.77 \quad 43.24$

1.00
0.96
0.66
1.16
0.00
0.30
0.83
0.22
0.83
0.86
2.98
0.45
0.00
1.28

1.00

0.02

HIGH HEAT- ERROR REF

MEAS CALC (M-C)/

M 
NAME

Corn Stover

Corn Stover

Corncobs

Cotton gin trash

Cotton gin waste

Cotton Stalk

Cotton Stalk

English Walnut Prunings

Grape pomace

Groundnut shells

Macadamia Śhjells

Millet Straw

Napier Grass

Olive Pits

Peach Pits

Peach Pits

Peanut hulls

Pine needles

Pistachio Shells

Rice Hulls

Rice Husk (Patni-23)

\section{PROXIMATE}

Fixed Vola- Ash

Carbon tiles

$\%$

$\% \quad \%$

$\begin{array}{ll}19.25 & 75.17 \\ 14.50 & 78.10 \\ 18.54 & 80.10 \\ 15.10 & 67.30 \\ 14.97 & 83.41 \\ 19.90 & 62.90 \\ 22.43 & 70.89 \\ 18.10 & 80.82 \\ 21.40 & 74.40 \\ 21.60 & 72.70 \\ 23.68 & 75.92 \\ 16.45 & 78.28 \\ & \\ 21.20 & 75.60 \\ 19.85 & 79.12 \\ 19.80 & 79.10 \\ 21.09 & 73.02 \\ 26.12 & 72.38 \\ 16.84 & 82.03 \\ 15.80 & 63.60 \\ 14.90 & 69.30\end{array}$

$\%$

\section{ULTIMATE ANALYSIS}

\section{$\begin{array}{llll}C & \mathbf{H} & \mathbf{O} & \mathbf{N}\end{array}$}

AGRICULTURAL (Cont)

\section{$\begin{array}{llll}5.58 & 43.65 & 5.56 & 43.31\end{array}$}

$\begin{array}{llll}7.40 & 46.50 & 5.81 & 39.67\end{array}$

$\begin{array}{lllll}1.36 & 46.58 & 5.87 & 45.46\end{array}$

$\begin{array}{llll}17.60 & 39.59 & 5.26 & 36.38\end{array}$

\begin{tabular}{ll}
$1.61 \quad 42.66$ \\
\hline
\end{tabular}

$\begin{array}{ll}17.20 & 39.47\end{array}$

$\begin{array}{ll}6.68 & 43.64\end{array}$

$\begin{array}{ll}1.08 & 49.72\end{array}$

$\begin{array}{ll}4.20 & 54.94\end{array}$

$5.70 \quad 48.59$

$\begin{array}{ll}0.40 & 54.41\end{array}$

$\begin{array}{lll}5.27 & 43.71\end{array}$

$5.70 \quad 45.20$

$3.20 \quad 48.81$

$1.03 \quad 53.00$

$\begin{array}{ll}1.10 & 49.14\end{array}$

$\begin{array}{ll}5.89 & 45.77\end{array}$

$\begin{array}{ll}1.50 & 48.21\end{array}$

$\begin{array}{ll}1.13 & 48.79\end{array}$

$20.60 \quad 38.30$

$15.80 \quad 38.92$
$6.05 \quad 49.50$

$\begin{array}{ll}5.07 & 38.09\end{array}$

$\begin{array}{ll}5.81 & 43.87\end{array}$

$\begin{array}{ll}5.63 & 43.14\end{array}$

$\begin{array}{ll}5.83 & 32.08\end{array}$

\begin{tabular}{ll}
$5.64 \quad 39.49$ \\
\hline
\end{tabular}

$\begin{array}{ll}4.99 & 39.69\end{array}$

$5.85 \quad 45.16$

$6.00 \quad 42.30$

$\begin{array}{ll}6.23 & 43.48\end{array}$

$\begin{array}{ll}5.90 & 39.14\end{array}$

$\begin{array}{ll}6.34 & 43.52\end{array}$

$\begin{array}{lll}5.46 & 39.56\end{array}$

$\begin{array}{lll}5.91 & 43.41\end{array}$

$4.36 \quad 35.45$

$\begin{array}{ll}5.10 & 37.89\end{array}$ $\begin{array}{ll}6.57 & 43.72\end{array}$
$\%$

\subsection{1}

0.56

0.47

2.09

0.18

1.25

0.00

0.37

2.09

0.58

0.36

0.01

0.00

0.36

0.32

0.48

1.63

0.56

0.83

2.17
HIGH HEAT- ERROR REF

MEAS CALC (M-C)/

M

$\% \quad \mathrm{~kJ} / \mathrm{mol} \mathrm{kJ} / \mathrm{mol}$

$\begin{array}{lllll}0.01 & 17.65 & 17.19 & 2.63 \% & 6\end{array}$

$\begin{array}{lllll}0.11 & 19.00 & 18.82 & 0.91 \% & 21\end{array}$

$\begin{array}{lllll}0.01 & 18.77 & 18.44 & 1.75 \% & 6\end{array}$

$\begin{array}{lllll}0.00 & 16.42 & 15.85 & 3.45 \% & 6\end{array}$

$\begin{array}{llll}17.48 & 16.87 & 3.53 \% & 5\end{array}$

$\begin{array}{lllll}0.02 & 15.83 & 15.44 & 2.50 \% & 5\end{array}$

$\begin{array}{llllll}0.00 & 18.26 & 17.40 & 4.69 \% & 12\end{array}$

$\begin{array}{lllll}0.01 & 19.63 & 19.50 & 0.65 \% & 6\end{array}$

$\begin{array}{lllll}0.21 & 21.80 & 22.63 & -3.81 \% & 11\end{array}$

$\begin{array}{llll}19.85 & 19.40 & 2.28 \% & 20\end{array}$

$\begin{array}{lllll}0.01 & 21.01 & 20.76 & 1.20 \% & 6\end{array}$

$\begin{array}{lllll}0.00 & 18.05 & 17.37 & 3.75 \% & 5\end{array}$

$\begin{array}{llll}18.31 & 18.35 & -0.26 \% & 23\end{array}$

$\begin{array}{lllll}0.01 & 21.39 & 19.81 & 7.36 \% & 5\end{array}$

$\begin{array}{lllll}0.05 & 20.82 & 21.39 & -2.72 \% & 6\end{array}$

$\begin{array}{lllll}0.02 & 19.42 & 20.10 & -3.47 \% & 11\end{array}$

$\begin{array}{lllll}0.12 & 18.64 & 18.18 & 2.45 \% & 6\end{array}$

$\begin{array}{llll}20.12 & 20.02 & 0.50 \% & 12\end{array}$

$\begin{array}{llllll}0.01 & 19.26 & 19.48 & -1.12 \% & 6\end{array}$

$\begin{array}{lllll}0.06 & 14.89 & 14.40 & 3.26 \% & 11\end{array}$

$\begin{array}{lllll}0.12 & 15.67 & 15.32 & 2.23 \% & 3\end{array}$ 
NAME

Rice Husk Bran

Rice Straw

Sugarcane Bagasse

Walnut Shells

Wheat dust

Wheat Straw

Brown Kelp, Giant, Monterey

Brown Kelp, Soquel Point

Water Hyacinth

Water Hyacinth (Florida)

Cedar

Douglas Fir bark

Loblolly Pine bark

Long Leaf Pine bark

Slash Pine bark

Spruce
PROXIMATE

Fixed Vola- Ash

Carbon tiles

$\% \quad \%$

$\begin{array}{ll}19.53 & 61.83 \\ 17.25 & 69.33 \\ 14.95 & 73.78 \\ 21.16 & 78.28 \\ 16.47 & 69.85 \\ 19.80 & 71.30\end{array}$

$\%$

\section{ULTIMATE ANALYSIS}

\section{$\begin{array}{lllll}\mathbf{C} & \mathbf{H} & \mathbf{O} & \mathbf{N} & \mathbf{S}\end{array}$}

HIGH HEAT- ERROR REF

AGRICULTURAL (Cont)

\section{$\begin{array}{llll}18.64 & 38.92 & 5.12 & 36.77\end{array}$}

$\begin{array}{lllll}13.42 & 41.78 & 4.63 & 36.57\end{array}$

$\begin{array}{llll}11.27 & 44.80 & 5.35 & 39.55\end{array}$

$\begin{array}{llll}0.56 & 49.98 & 5.71 & 43.35\end{array}$

$\begin{array}{llll}13.68 & 41.38 & 5.10 & 35.17\end{array}$

$\begin{array}{llll}8.90 & 43.20 & 5.00 & 39.40\end{array}$

\section{AQUATIC BIOMASS}

54.20

$\begin{array}{llll}45.80 & 26.60 & 3.74 & 20.22\end{array}$

57.90

$\begin{array}{llll}42.10 & 27.80 & 3.77 & 23.69\end{array}$

$\begin{array}{llll}15.30 & 43.00 & 5.80 & 29.50\end{array}$

80.40

$4.60 \quad 33.99$

\section{BARK}

$\begin{array}{llllll}21.90 & 73.00 & 5.10 & 51.00 & 5.70 & 38.20\end{array}$

$\begin{array}{llllll}25.80 & 73.00 & 1.20 & 56.20 & 5.90 & 36.70\end{array}$

$\begin{array}{llllll}33.90 & 54.70 & 0.40 & 56.30 & 5.60 & 37.70\end{array}$

$32.30 \quad 67.00$

$\begin{array}{llll}0.70 & 56.40 & 5.50 & 37.40\end{array}$

$0.70 \quad 56.20$

$5.40-37.30$

$33.40 \quad 56.90$

$\begin{array}{llll}3.80 & 51.80 & 5.70 & 38.60\end{array}$

\section{$\% \quad \% \quad \mathrm{~kJ} / \mathrm{mol} \quad \mathrm{kJ} / \mathrm{mol}$}

\section{$0.55 \quad 0.00$}

$\begin{array}{lll}0.70 & 0.08\end{array}$

$\begin{array}{ll}0.38 & 0.01\end{array}$

$\begin{array}{ll}0.21 & 0.01\end{array}$

$\begin{array}{lll}3.04 & 0.19\end{array}$

0.61

0.11

$\begin{array}{ll}2.55 & 1.09\end{array}$

$\begin{array}{ll}4.63 & 1.05\end{array}$

5.60

151

0.00

\subsection{6}

10.75

17.98

14.86

20.03

$\begin{array}{lll}0.00 & 0.00\end{array}$

$\begin{array}{ll}0.00 & 0.00\end{array}$

0.00

0.00

0.10
$22.10-22.75$

$\begin{array}{ll}21.78 & 22.35\end{array}$

$\begin{array}{ll}21.77 & 22.29\end{array}$

$21.77 \quad 22.11$

$20.33 \quad 20.74$
(M-C)/

M

$-0.82 \% \quad 5$

$1.88 \% \quad 6$

$-1.63 \% \quad 6$

$2.48 \% \quad 6$

$-1.86 \% \quad 6$

$4.55 \% \quad 6$

$\begin{array}{lll}10.71 & -4.38 \% & 18\end{array}$

$10.85 \quad-0.94 \% \quad 18$

$18.39-2.26 \% \quad 23$

$15.54 \quad-4.56 \% \quad 17$

$20.46 \quad-2.17 \% \quad 20$

$-2.96 \% \quad 24$

$-2.61 \%$

$-2.37 \% \quad 16$

$-1.56 \% \quad 16$

$-2.01 \% \quad 20$ 
NAME

Casuarina

Casuarina

Eucalyptus Camaldulensis

Eucalyptus Globulus

Eucalyptus Grandis

Poplar

Sudan Grass

尜

\section{HYDROCARBONS}

Benzene, C6H6

Diesel Oil

Kerosene

Methane, CH4

Motor Gasoline

n-octane

No. 6 Fuel Oil

ALCOHOLS

Ethanol, C2H5OH

Methanol, $\mathrm{CH} 3 \mathrm{OH}$

\section{PROXIMATE}

Fixed Vola- Ash

\section{Carbon tiles}

$\%$

$\%$

$19.66 \quad 78.94$

$19.58 \quad 78.58$

$\begin{array}{ll}17.82 & 81.42\end{array}$

$17.30 \quad 81.60$

$16.93 \quad 82.55$

$16.35 \quad 82.32$

$18.60 \quad 72.75$

\section{ULTIMATE ANALYSIS}

C

$\%$

\section{ENERGY CROPS}

$\begin{array}{rrrrrrrrrr}1.40 & 48.61 & 5.83 & 43.36 & 0.59 & 0.02 & 19.44 & 19.32 & 0.62 \% & 6 \\ 1.83 & 48.50 & 6.04 & 43.32 & 0.31 & 0.00 & 18.77 & 19.53 & -4.03 \% & 5 \\ 0.76 & 49.00 & 5.87 & 43.97 & 0.30 & 0.01 & 19.42 & 19.46 & -0.19 \% & 6 \\ 1.10 & 48.18 & 5.92 & 44.18 & 0.39 & 0.01 & 19.23 & 19.20 & 0.16 \% & 6 \\ 0.52 & 48.33 & 5.89 & 45.13 & 0.15 & 0.01 & 19.35 & 19.13 & 1.12 \% & 6 \\ 1.33 & 48.45 & 5.85 & 43.69 & 0.47 & 0.01 & 19.38 & 19.26 & 0.64 \% & 6 \\ 8.65 & 44.58 & 5.35 & 39.18 & 1.21 & 0.01 & 17.39 & 17.62 & -1.30 \% & 6\end{array}$

\section{LIQUID FUELS}

$\begin{array}{rrrrrrrrr}92.25 & 7.75 & 0.00 & 0.00 & 0.00 & 41.79 & 41.34 & 1.08 \% & 8 \\ 86.50 & 13.20 & 0.00 & 0.00 & 0.30 & 45.70 & 45.78 & -0.18 \% & 8 \\ 85.80 & 14.10 & 0.00 & 0.00 & 0.10 & 46.50 & 46.58 & -0.17 \% & 8 \\ 74.85 & 25.15 & 0.00 & 0.00 & 0.00 & 55.35 & 55.76 & -0.76 \% & 8 \\ 85.50 & 14.40 & 0.00 & 0.00 & 0.10 & 46.88 & 46.83 & 0.12 \% & 10 \\ 84.10 & 15.90 & 0.00 & 0.00 & 0.00 & 47.80 & 48.09 & -0.63 \% & 8 \\ 85.70 & 10.50 & 1.70 & 2.00 & 0.05 & 42.30 & 42.09 & 0.50 \% & 14 \\ & & & & & & & & \\ 52.20 & 13.00 & 34.80 & 0.00 & 0.00 & 30.15 & 29.94 & 0.69 \% & 8 \\ 37.50 & 12.50 & 50.00 & 0.00 & 0.00 & 22.69 & 22.65 & 0.18 \% & 8\end{array}$




\section{NAME}

\section{PYROLYSIS OILS}

BOM wood oil

Coke-oven tar

LBL Wood Oil

Low Temp Tar

\section{COAL}

के German Braunkole Lignite

German-Anna

Green In. \#3

Nurthumberland No 8 Anthr.

Peat, S-H3

Pittsburgh Seam

Wyoming Elkol

COKE

Coke

Low Temperature Coke
PROXIMATE

Fixed Vola- Ash

Carbon tiles

$\%$

$\%$

$\%$

$\begin{array}{ll}0.66 & 82.00\end{array}$

$\begin{array}{lll}0.25 & 91.75 & 5 \\ 0.78 & 1.30 & 8\end{array}$

$0.78 \quad 72.30$

83.00

\section{ULTIMATE ANALYSIS}

HIGH HEAT- ERROR REF

$\begin{array}{llll}\mathbf{H} & \mathbf{0} & \mathbf{N} & \mathbf{S}\end{array}$

MEAS CALC (M-C)/

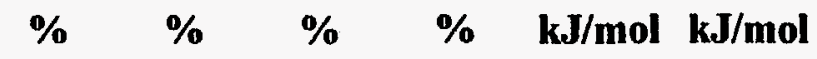

M

$\begin{array}{rrrrrrrc}8.80 & 9.20 & 0.60 & 0.00 & 36.80 & 38.02 & -3.32 \% & 14 \\ 5.50 & 0.80 & 0.90 & 0.80 & 38.20 & 38.49 & -0.76 \% & 8 \\ 8.60 & 17.60 & 0.20 & 0.01 & 33.70 & 33.54 & 0.49 \% & 14 \\ 8.20 & 7.40 & 0.60 & 0.80 & 38.75 & 37.94 & 2.08 \% & 8\end{array}$

\section{SOLID FUELS}

$\begin{array}{rrrrrrrrrrrr}46.03 & 49.47 & 4.50 & 63.89 & 4.97 & 24.54 & 0.57 & 0.48 & 25.10 & 25.57 & -1.86 \% & 1 \\ 79.60 & 12.00 & 8.40 & 82.62 & 3.02 & 3.66 & 0.92 & 0.73 & 33.00 & 31.91 & 3.32 \% & 1 \\ 41.53 & 40.93 & 17.55 & 62.70 & 4.84 & 6.29 & 1.36 & 7.17 & 27.36 & 27.27 & 0.31 \% & 4 \\ 84.59 & 7309.0 & 8.32 & 83.67 & 3.56 & 2.84 & 0.55 & 1.05 & 32.86 & 33.03 & -0.54 \% & 4 \\ 26.87 & 70.13 & 3.00 & 54.81 & 5.38 & 35.81 & 0.89 & 0.11 & 22.00 & 21.71 & 1.34 \% & 19 \\ 55.80 & 33.90 & 10.30 & 75.50 & 5.00 & 4.90 & 1.20 & 3.10 & 31.75 & 31.82 & -0.21 \% & 15 \\ 46.60 & 43.00 & 4.20 & 71.50 & 5.30 & 16.90 & 1.20 & 0.90 & 29.57 & 29.44 & 0.42 \% & 15 \\ & & & & & & & & & & & 1 \\ 91.47 & 0.92 & 7.61 & 89.13 & 0.43 & 0.98 & 0.85 & 1.00 & 31.12 & 31.45 & -1.04 \% & 10 \\ 81.79 & 11.15 & 7.06 & 83.53 & 2.82 & 3.76 & 1.00 & 1.82 & 30.94 & 32.11 & -3.79 \% & 8\end{array}$


NAME

CHARCOAL

Casuarina Char (950C)

Charcoal

Coconut Shell Char (750C)

Eucalyptus char (950C)

Oak char (565C)

Oak char (630C)

Redwood char (550C)

$\stackrel{?}{i}$

Redwood char (935C)

Subabul wood char (950C)

Acetylene

Carbon

Carbon dioxide, $\mathrm{CO} 2$

Carbon Monoxide, $\mathrm{CO}$

Hydrogen, $\mathrm{H} 2$

Water

\section{PROXIMATE}

Fixed Vola- Ash

Carbon tiles

$\%$

$89.31 \quad 93.88 \quad 1.02$

87.179393 .0

$\begin{array}{ll}70.32 & 19.22\end{array}$

$\begin{array}{ll}55.60 & 27.10\end{array}$

$59.30 \quad 25.80$

$67.70 \quad 30.00$

$72.00 \quad 23.90$

$74.50 \quad 21.65$

17.30

14.90

3.95

\section{ULTIMATE ANALYSIS}

C H

$\mathbf{N}$

$\mathbf{S}$

HIGH HEAT- ERROR REF

MEAS CALC (M-C)/

$\mathbf{M}$

$\begin{array}{lllllll}\% & \% & \% & \% & \% & \mathrm{~kJ} / \mathrm{mol} & \mathbf{k J} / \mathrm{mol}\end{array}$

$\begin{array}{lll}77.54 & 0.93 \quad 5.62\end{array}$

$1.02 \quad 92.04$

$2.90 \quad 88.95$

$10.45 \quad 76.10$

2.45

0.73

1.38

$\begin{array}{lll}1.33 & 11.10 & 1.02\end{array}$

64.60

2.10

15.50

0.40

$2.30 \quad 75.60$

$4.10 \quad 78.80$

$2.40 \quad 14.40$

0.40

$\begin{array}{lll}3.30 & 18.40 & 0.20\end{array}$

$\begin{array}{lll}3.50 & 1320 & 0.20\end{array}$

\section{EXCEPTIONS}

$\begin{array}{ccrlrrrrr}92.25 & 7.75 & 0.00 & 0.00 & 0.00 & 49.60 & 41.34 & 16.66 \% & 7 \\ 100.0 & & & & & 32.81 & 34.91 & -6.40 \% & 7 \\ 27.27 & & & & & 0.00 & 9.52 & & 7 \\ 42.86 & 0.00 & 57.14 & 0.00 & 0.00 & 10.16 & 9.05 & 10.89 \% & 7 \\ 0.00 & 100.0 & 0.00 & 0.00 & 0.00 & 141.26 & 117.83 & 16.59 \% & 7 \\ 0.00 & 11.11 & 88.89 & & & 0.00 & 3.90 & & 7\end{array}$

\section{NOTES:}

Data taken primarily from [5], Channiwala, Salim A., "On Biomass Gasification Process and Technology Development", PhD Thesis, Indian In of Techn., Bombay, 1992. 
1 Bliek, A., "Mathematical Modelling of a Co-Current Fixed Bed Coal Gasifier", Ph.D. Thesis, Twente University of Technology, Eindhoven, The Netherlands, 1984.

2 Jenkins, B. M., "Downdraft Gasification Characteristics of Major California Residue Derived Fuels", Ph.D. Thesis, Univ. of California, Davis, (1980).

3 Maheshwari, R. C., "Utilizatyion of Rice Husk as Fuel" Ph.D. Thsdis, Agricultural Engineering Dept., IIT, Kharagpur, India, (1975).

4 IGT, Chicago, III, "Preparation of Coal Conversion Systems", Technical Data Book, Project 8964 Final Report, Contract No. E (49-18), (1976).

5 Channiwala, Salim A., "On Biomass Gasification Process and Technology Development", PhD Thesis, Indian Inst. of Techn., Bombay, 1992.

6 Jenkins, B. M. and Ebeling, J. M., "Correlation of Physical and Chemical Properties of Terrestrial Biomass with Conversion:, Symposium, Energy From Biomass and Waste IX, IGT, p. 271 (1985).

7 Stull, D. R. and Prophet, H., "JANAF Thermochemical Tables, NSRDS-NBS-37, Washington, 2nd Ed., 1971).

8 Rose, J. W. and Cooper, J. R. (Ed), "Techical Data on Fuel", the British National Committee of the World Energy Conference, Edingurgh, 7th Ed., (1977).

9 Gaur, S. and Reed, T. B., this work

10 Spiers, H. M., "Technical Data on Fuel", John Wiley, N. Y. 6th Edition, (1962).

11 Rossi, A., "Fuel Characteristics of Wood and Non-Wood Biomass Fuels", in Progress in Biomass Conversin, Ed. Tillman, D. A. and Jahn, E. C>, Vol 5, Academic Press, Inc., pp 69; (1984).

12 Grover, P. D. and Anuradha, G., "Thermochemical Characterization of Biomass for Gasirication", Report on Physico-CHemical Parameters of Biomass Residues, IIT-Delhi (1988).

13 Boley, E. C. and Landers, W. S., "Entrainment Drying and Carbonizatin of Wood Waste", Report of Investigations 7282, Bureau of Mines, Washington, D>C. (1969).

14 Elliot, D. C., "Process Development for Direct Liquefaction of Biomass", in Fuels from Biomass and Wastes, Ed. Klass, L. D. and Emmert, G. H., Ann Arbor Science, pp. 435-450 (1981).

15 Bittuminous Coal Research, Inc., "Gas Generator Research and Development", Phase II - Process and Equipment Development, Contract No. OCR-20-F, 235530/3GI, (1974).

16 Risser, P.G., "Agricultural and Forestry Residues", in Biomass Conversion Processes for Energy and Fuels, Ed. Soffer, S. S. and Zaborsky, O. R., Plenum Press, N.Y., pp 25-56d (1981).

17 Klass, D. L. and Ghosh, S., "Methane Production by anaerobic Digestion of Water Hyacinth", in Fuels from Biomass and Wastes, Ed., Klass, L. D. and Emmert, O.H., Ann Arbor, pp. 129-149 (1981). 
18 Chynoweth, D. P., Klass, D. L. and Ghosh, S. "Biomethanation of Giant Brown Kelp-Macrocystis Pyrifera", in Energy from Biomass and Wastes, IGT, Washington, pp. 229-252 (1978).

19 Aho, M., "A Comparison of the Composition, Pyrolysis and Burning Properties of Indonesian and Finnish Peat", in Research in Thermochemical Biomass Conversin, Ed., Bridgwater, A. V. and Kuester, J. L., Elsevier, N.Y., pp. 203-213 (1988).

20 Johnson, J. A. and Auth, G. H., "Fuels and Combustion Handbook", McGraw-Hill, N.Y., (1951).

21 Gregory, M., Rose, G. R., Nandi, S. P., Onischak, M., Zabransky, R. F. and Babu, S. R., "Substitute Fuel", Proc. 14th Biomass Thermochemical Conversion Contractor's Meeting, pp 71-147 (1982).

22 Mudge, L.K., Mitchell, D.H., Baker, E.G., Robertus, R. J. and Brown, M.D., "Steam Gasification of Wood in the Presence of Catalysts", Proc. 14th Biomass Thermochemical Conversion Contractor's Meeting, pp 9-31 (1982).

23 Elliot, D.C., Butner, S. and Sealock, L.J. (Jr.), "Low-Temperature Gasification of High-Moisture Biomass", in Research in thermochemical Biomass Conversin, Eds Bridgwater, A.V. and Kuester, J.L., Elsevier, N.Y., pp 696-710 (1988).

Tillman, D. A., "Wood as an Energy Resource", Academic Press, N. Y., (1978). 


\section{APPENDIX B}

(Sources of samples)

Sample name Source

Acetylated xylan NREL

Avicel PH 102 CSM

Babool wood CSM

Baggase. NREL

Biodiesel CSM

Biomass components additivity (populus deltoides) NREL

Black locust CSU

Brown rotten wood FPL

Cardboard, Corregating sheet IPST

Cardboard, Liner board IPST

Cellulsse (Baker analyzed)

CSM

Cellulose (Doped with $0.1 \% \mathrm{KOH}$ ) NREL

Cellulose (Doped with $0.1 \% \mathrm{ZnCl}_{2}$ )

NREL

Cellulose (Synthetic). CSM

Cellulose, Prima rayonier

CSU

Coal, Blind canyon

ANL

Coal, Buelah zap lignite (North Dakota)

ANL

Coal, Illinois \# 6

ANL

Coal, Pittsburg \# 8

ANL

Coal, Wyodak bituminus

ANL

Coconut shell.

CSM

Corn oil

CSM

Corn oil methyl ester.

CSM

Cotton (dryland)

NREL

Cotton (Irrigated)

NREL

Diesel fuel

CSM

Eastern red cedar.

CSU

Ecofuel II

NREL

Eelgrass. MRI

Giant kelp MRI

Glucose. CSM

Glycerol CSM

Lignin (Populus deltoides) NREL

Lignin, Kraft pine. NREL

Lignin-aspen (Steam exploded) NREL

Lodgepole pine CSU

Oak bark . CSU 
Oak Pyrolysis oil

NREL

Osage orange CSU

Paper, 25\% Cotton mix

Paper, Kimwipe tissue

CSM

Paper, Newsprint IPST

Paraffin

CSM

Peach seed

CSM

Peanut shell

CSM

Peat (Steam exploded)

IHI

Peat, Pahokee Fla

IHI

Monterey Pine (Pinus radiata)

CSU

Pistachio nut CSM

Plant Anatomy samples

NREL

Plant extract samples

NREL, CSM

Plant Maturation samples

NREL

Polydimethyl siloxane CSM

Polyethylene

CSM

Polyethylene glycol.

CSM

Polyhydroxy benzoic acid

CSM

Polymethyl methacrylate

CSM

Polystyrene

CSM

Polytetrafluroethylene (Teflon)

CSM

Polyvinyl acetate.

CSM

Polyvinyl chloride

CSM

Ponderesa pine

CSU

Ponderesa pine bark.

CSU

Poplar (stored for 26 weeks).

NREL

Poplar (stored for zero week)

NREL

Populus deltoidus

NREL

Populus tremeloid

NREL

Pumpkin seed

CSM

Red alder bark

CSM

Refuse derived fuel (Terylene)

NREL

Refuse derived fuel (Thief river falls).

NREL

Rice hull.

CSM

Rubber tire (Pyrolysis oil)

NREL

Rubber wood

NREL

Sargassum weed

MRI

Sericea

NREL

Sericea (stored for 26 weeks)

NREL

Sericea (stored for zero week)

NREL

Slash pine CSU

Sorghum (stored for 26 weeks).

NREL

Sorghum (stored for zero week)

NREL 
Spinach.

NREL

Sugar pine

CSU

Sunflower stalk CSM

Switch grass (Pyrolysis oil) NREL

Switch grass (stored for 26 weeks). NREL

Switch grass (stored for zero week)

Switch grass char NREL

Walnut NREL

Waste vegetable oil ester CSM

Western Hemlock CSM

Western red ceder CSU

Wheat straw. CSU

White rotten wood CSM FPL

ANL: Argonne National Laboratory, Argonne, Illinois.

CSM: Colorado School of Mines, Golden, Colorado, contact person: Drs. T. B. Reed, S. Gaur, R. Baldwin

CSU: Colorado State University, Fort Collins, Colorado, contact person: Dr. H. Schroeder

F.PL: U.S. Forest Products Laboratory, Dr. Terry Highly, Madison, WI.

IPST: Institute of Paper Science and Technology, Atlanta, Georgia, contact person: A. W. Rudie

MRI: Marine Research Institute, Woods Hole, MA.

NREL: National Renewable Energy Laboratory, Golden, Colorado, Contact persons: F. Agblevaor, R. Evans, D. Johnson, J Diebold, T. Milne 


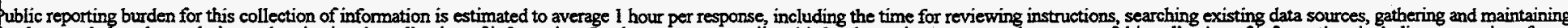

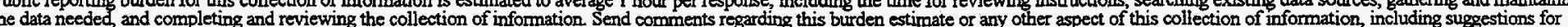

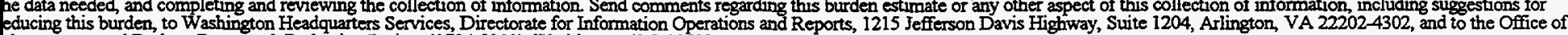
Management and Budget, Paperwork Reduction Project (0704-0188), Washington, DC 20503.

\begin{tabular}{|l|c|c|}
\hline $\begin{array}{l}\text { AGENCY USE ONLY (Leave } \\
\text { lank) }\end{array}$ & $\begin{array}{c}\text { 2. REPORT DATE } \\
\text { May } 1995\end{array}$ & $\begin{array}{c}\text { 3. REPORT TYPE AND DA } \\
\text { Final subcontract report }\end{array}$ \\
\hline
\end{tabular}

TITLE AND SUBTITLE

An Atlas of Thermal Data for Biomass and Other Fuels

Final Report

AUTHOR(S)

Siddhartha Gaur, and Thomas Reed

PERFORMING ORGANIZATION NAME(S) AND ADDRESS(ES)

\section{FUNDING NUMBERS}

(C) HZ-2-12083-1

(TA)BF52.4012

SPONSORING/MONITORING AGENCY NAME(S) AND ADDRESS(ES)

Jational Renewable Energy Laboratory

617 Cole Boulevard

folden, CO 80401-3393

\section{PERFORMING \\ ORGANIZATION \\ REPORT NUMBER}

DE95009212

10.

SPONSORING/MONITORING

AGENCY REPORT

NUMBER

NREL/TP-433-7965

1. SUPPLEMENTARY NOTES

2a. DISTRIBUTION/AVAILABILITY STATEMENT

Jational Technical Information Service

J.S. Department of Commerce

285 Port Royal Road

pringfield, VA 22161 12b. DISTRIBUTION CODE

UC-1310

\section{ABSTRACT (Maximum 200 words)}

This atlas presents thermogravimetric and differential thermal analysis data taken under comparable conditions on a wide variety of rganic materials such as biomass fuels, coal and municipal solid wastes making it easy to find and compare data on this large variety of energy ources. Some data has been collected by changing the operating conditions to show the effects of parameters such as particle size, heating rate tc. on the decomposition pattern of a solid sample. This information can then be used to provide a guideline for the extrapolation of the bulk $\mathrm{f}$ the data and data previously available in the literature but obtained at different conditions.

\begin{tabular}{|c|c|c|c|}
\hline \multirow{2}{*}{\multicolumn{3}{|c|}{$\begin{array}{l}\text { 4. SUBJECT TERMS } \\
\text { hemical reactions, biomass, feedstocks, process controls, novel process development, gasification, } \\
\text { yrolysis oil. }\end{array}$}} & \multirow{2}{*}{$\begin{array}{l}\text { 15. NUMBER OF PAGES } \\
\frac{187}{16 . \text { PRICE CODE }} \\
\text { A09 }\end{array}$} \\
\hline & & & \\
\hline $\begin{array}{l}\text { 7. SECURITY } \\
\text { LASSIFICATION } \\
\text { OF REPORT } \\
\text { Inclassified }\end{array}$ & $\begin{array}{l}\text { 18. SECURITY } \\
\text { CLASSIFICATION } \\
\text { OF THIS PAGE }\end{array}$ & $\begin{array}{l}\text { 19. SECURITY } \\
\text { CLASSIFICATION } \\
\text { OF ABSTRACT }\end{array}$ & $\begin{array}{l}\text { 20. LIMITATION OF } \\
\text { ABSTRACT }\end{array}$ \\
\hline & & & \\
\hline & & & \\
\hline & & & \\
\hline
\end{tabular}

